\title{
Advancing the cell culture landscape
}

\author{
Citation for published version (APA):
}

Vermeulen, S. A. (2020). Advancing the cell culture landscape: the instructive potential of artificial and natural geometries. [Doctoral Thesis, Maastricht University]. Maastricht University. https://doi.org/10.26481/dis.20200904sv

Document status and date:

Published: 01/01/2020

DOI:

10.26481/dis.20200904sv

Document Version:

Publisher's PDF, also known as Version of record

\section{Please check the document version of this publication:}

- A submitted manuscript is the version of the article upon submission and before peer-review. There can be important differences between the submitted version and the official published version of record.

People interested in the research are advised to contact the author for the final version of the publication, or visit the DOI to the publisher's website.

- The final author version and the galley proof are versions of the publication after peer review.

- The final published version features the final layout of the paper including the volume, issue and page numbers.

Link to publication

\footnotetext{
General rights rights.

- You may freely distribute the URL identifying the publication in the public portal. please follow below link for the End User Agreement:

www.umlib.nl/taverne-license

Take down policy

If you believe that this document breaches copyright please contact us at:

repository@maastrichtuniversity.nl

providing details and we will investigate your claim.
}

Copyright and moral rights for the publications made accessible in the public portal are retained by the authors and/or other copyright owners and it is a condition of accessing publications that users recognise and abide by the legal requirements associated with these

- Users may download and print one copy of any publication from the public portal for the purpose of private study or research.

- You may not further distribute the material or use it for any profit-making activity or commercial gain

If the publication is distributed under the terms of Article $25 \mathrm{fa}$ of the Dutch Copyright Act, indicated by the "Taverne" license above, 


\section{Advancing the cell culture landscape: the instructive potential of artificial and natural geometries}


The research described in this thesis has received funding from the European Union's Horizon 2020 research and innovation programme under the Marie Skłodowska-Curie grant agreement No 676338 .

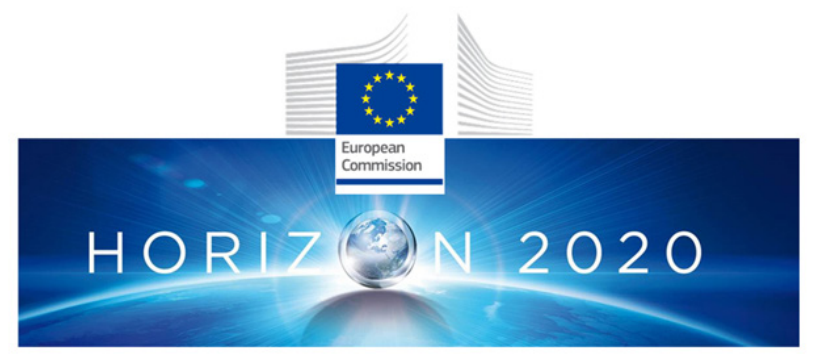

The printing of this thesis was sponsored by:

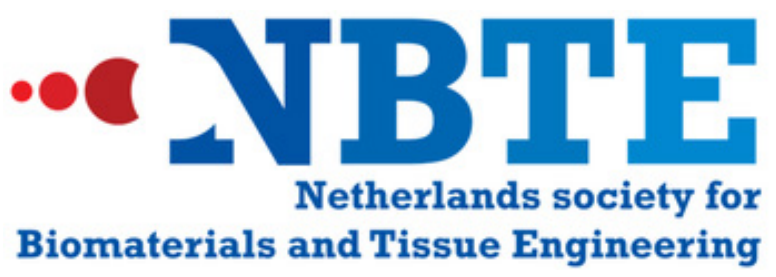

Copyright 2020 (C) Steven Vermeulen, Maastricht

Advancing the cell culture landscape: the instructive potential of artificial and natural geometries $\mathrm{PhD}$ Thesis, Maastricht University, Maastricht, the Netherlands

ISBN: 978-94-6380-886-6

Cover design: Heidi Wiigaerts

Printed by: ProefschriftMaken

All rights are reserved. For articles published, the copyright has been transferred to the respective publisher. No parts of this thesis may be reproduced, stored in a retrieval system or transmitted in any form or by any means without prior permission from the author. 


\title{
Advancing the cell culture landscape: the instructive potential of artificial and natural geometries
}

\author{
DISSERTATION
}

To obtain the degree of Doctor at the Maastricht University, on the authority of the Rector Magnificus,

Prof.dr. Rianne M. Letschert

in accordance with the decision of the Board of Deans, to be defended in public on Friday, $4^{\text {th }}$ of September 2020 at 14:00 by

Steven Albert Vermeulen 


\section{Promotor:}

Prof. Dr. Jan de Boer

Co-promotor:

Dr. Aurélie Carlier

\section{Assessment committee:}

Prof. Dr. Pamela Habibović (Chair)

Prof. Dr. Clemens van Blitterswijk

Prof. Dr. Carlijn Bouten

Dr. Pieter Emans

Prof. Dr. Amir Ghaemmaghami, University of Nottingham, United Kingdom 


\section{Table of Contents}

Chapter I

General Introduction

Chapter II

Shaping Cell Fate: influence of Topographical Substratum Properties on Embryonic Stem Cells

Chapter III

Screening as a Strategy to drive Regenerative Medicine Research

Chapter IV

67

Identification of Topographical Architectures Supporting the Phenotype of Rat Tenocytes

Chapter $V$

Mechanotransduction is a Context-Dependent Activator of TGF-B Signaling in Mesenchymal Stem Cells

Chapter VI

The Covalent State of Immobilized RGD Peptides dictates the Mechanobiological Response of Fibroblasts

Chapter VII

Epigenetic Regulation of Mesenchymal Stem Cells by Micro-Topographies

Chapter VIII

Extending Biomaterial Surface Topographical Design Space through Natural Surface Reproduction

Chapter IX - Discussion 197

Chapter $X$ - Valorization 215

Summary 223

Samenvatting 224

Scientific Activities 225

Education Activities 229

Acknowledgments 231

Biography 


\section{Chapter I}

\section{General Introduction}

Steven Vermeulen and Jan de Boer 


\section{General Introduction}

Cells in the human body receive signals from a wide variety of sources. These can originate from the extracellular matrix (ECM), growth factors, the stiffness of the surrounding environment, mechanical forces, and geometrical cues. Cells will respond and change their behavior, depending on the input they receive. For example, when myocytes, the primary cell type found in muscles, are mechanically stimulated, they secrete growth factors such as IGF, leading to autocrine signaling and muscle growth ${ }^{1}$. Also, during developmental stages, fine-tuned mechanisms involving both mechanical and soluble cues allow for the differentiation of stem cells ${ }^{2}$. Understanding these mechanisms is beneficial for clinical applications, e.g., injecting pharmaceuticals for tissue repair. However, also in a tissue-engineering context where we aim to cultivate primary or pluripotent stem cells in sufficient quantities for the development of tissue constructs ex vivo. Here, we must adapt physiological conditions in the culture dish as close as possible to achieve the best tissue-engineering outcomes.

The main focus of the research outlined in this thesis is the use of surface structures in vitro for controlling cell behavior. Foremost, we use chapters II and III as review articles that introduce the experimental research performed in this thesis in a proper context. In chapter II, we highlight the diversity in surface structure design, and their potential to influence cell behavior ${ }^{3}$. We focus on pluripotent stem cells, which, due to their pluripotent capabilities, offer exciting clinical opportunities. Through chapter II, the beneficial effects of surface structures for cell culture applications should become apparent for the reader. However, due to the large variety in surface design, it is not easy assessing which surface structure type is most optimal for a particular application. Besides surface structures, the same challenge applies to other environmental perturbations such as the chemistry or stiffness of the material, the implementation of matrix proteins, or soluble cues. For example, concerning chemistry, already hundreds of different chemical compositions exist of polyurethane alone, a material applied in the clinic ${ }^{4}$. Therefore, high-throughput platforms were developed that allow screening hundreds of these environmental perturbations in one experimental setup, which is discussed in chapter III.

In chapter IV, we provide the reader with an example of the usefulness of such high-throughput platforms. Here, we utilized the TopoChip, a 2x2 cm Chip with 2176 unique micro-topographies, that helped us gain novel biological insights into cell-material interactions ${ }^{5}$. Tenocytes, the primary cell type of the tendon, rapidly lose phenotypic characteristics when put in standard culture conditions ${ }^{6}$. Through the TopoChip platform, we identified surface parameters that were associated with improved phenotypic characteristics, as shown by an elevation of the tendon transcription factor Scleraxis (SCX). In chapter V, we explore the biomechanics behind the phenomena. Here, we utilized human mesenchymal stem cells (MSCs) as a cell source and found again that micro-topographies could induce higher SCX levels. This phenomenon was associated with an activation of the mechanosensitive transcription factors SRF and EGR-1 during the early adaptation phase on micro-topographies, which coincided with an elevated and late expression of the TGF- $\beta$ receptor-II gene. We reasoned that this might indicate an increased sensitivity for TGF- $\beta$ signaling, which is also known for modulating SCX expression levels ${ }^{7}$. Therefore, we 8 
combined TGF- $\beta 2$ with micro-topographies and found synergistic effects on the expression levels of SCX and other TGF- $\beta$ responsive gene levels in MSCs, thereby gaining interesting insights on how signaling elicited by biomechanical and soluble cues converge.

In chapter VI, we continue down this path to further understand how biomechanical forces influence cell behavior. In this chapter, we employ surfaces functionalized with RGD peptides instead of the micro-topographies we previously used. RGD sequences are well-known peptide motifs found in multiple matrix proteins, including fibronectin and fibrinogen, and are known to interact with integrins leading to cellular signaling events ${ }^{8}$. Through cucurbit[8] uril chemistry, RGD peptides can either be covalent or non-covalently bound on the surface. In this study, we explored if the covalent state of these peptides can influence cell behavior. We found that surfaces coated with regular fibronectin, covalent, and non-covalent bound RGD peptides, provoked distinct cell behavior in NIH3T3 cells. Filopodia, cytoskeletal organization, and cell morphology were profoundly altered. Of interest, we identified through microarray and immunostaining experiments a higher presence of SRF and EGR1 during the early stages of cell attachment on the non-covalent bound RGD peptides compared to covalent bound RGD and regular fibronectin. Furthermore, this coincided with increased levels of fibrillary actin, thereby displaying strong similarities with the early induction of SRF and EGR1 on micro-topographies described in chapter $\mathbf{V}$.

In chapter VII, we delve deeper into the nuclear alterations evoked by micro-topographies. Previously, we found that micro-topographies altered actin cytoskeletal organization and subsequent biomechanical signaling that leads to increased TGF- $\beta$ sensitivity. However, we also know that nuclear morphology on micro-topographies is profoundly altered ${ }^{9}$, which is known to have a huge effect on cell behavior. For example, microgrooves can increase global histone acetylation levels in fibroblasts, which improves the efficiency of iPSCs transformation ${ }^{10}$. Therefore, we were interested in determining how micro-topographies alter the nuclear architecture in MSCs. For this, we applied histone mass-spectrometry 11 together with immunostainings, which revealed a global decrease in histone 3 and 4 acetylation. Mass spectrometry further pointed toward the differential presence of heat shock proteins, proteins associated with cytoskeletal and chromatin organization, and proteins associated with proliferation. We connected these findings to a "quiescent-like" phenotype, as demonstrated by the notion that MSCs after a month of culture on the micro-topographies retained their multipotent capabilities.

Since the surface structure design space is immense, even high-throughput platforms cannot encompass all architectural possibilities. We, therefore, reasoned that a platform that offers a very variable design diversity could lead to novel insights in cell-material interactions. For this, we looked at nature for inspiration since evolution enabled the design of intricate surface patterns for specific purposes. In chapter VIII, we discuss how we replicated 26 plant and insect surfaces in polystyrene to broaden our standard topographical design space with complex and hierarchical structures. Here, we found that MSCs cultured on this platform exhibited distinct 
cell morphological characteristics. In addition, we also found distinct bacterial attachment patterns on the artificial and natural topographies.

In the discussion chapter IX, we emphasize how the use of multiple environmental perturbations can eventually lead to improved biological outcomes in a tissue-engineering context. In the thesis, we highlight this concept in chapter $\mathbf{V}$, where we combine TGF- $\beta 2$ with micro-topographies, which results in improved expression levels of TGF- $\beta$ responsive genes. Here, we discuss similar observations. For example, adhesive islands influence the cells response against $\mathrm{TNF} \alpha{ }^{12}$, micro-wells reduce the inflammatory response against LPS ${ }^{13}$, while stretching stimulates the growth of smooth muscle cells through the upregulation of the EGF receptor ${ }^{14}$. These in vitro applications allow a glimpse of the in vivo environment where cells integrate a wide variety of signaling. Harnessing these concepts will lead to improved cell culture conditions for tissue engineering applications and disease models. Finally, we close off in the valorization chapter $\mathbf{X}$, where we highlight the possibility of commercializing the research findings described in this thesis.

\section{References}

1. Goldspink, D. F. et al. Muscle growth in response to mechanical stimuli. Am. J. Physiol. Endocrinol. Metab. 268, 288-297 (1995).

2. Vining, K. H. \& Mooney, D. J. Mechanical forces direct stem cell behaviour in development and regeneration. Nat. Rev. Mol. Cell Biol. 18, 728-742 (2017).

3. Kumari, S. et al. Shaping cell fate: influence of topographical substratum properties on embryonic stem cells. Tissue Eng. Part B Rev. 24, (2018).

4. Tare, R. S. et al. A microarray approach to the identification of polyurethanes for the isolation of human skeletal progenitor cells and augmentation of skeletal cell growth. Biomaterials 30, 1045-1055 (2009).

5. Vermeulen, S. et al. Identification of topographical architectures supporting the phenotype of rat tenocytes. Acta Biomater. 83, 277-290 (2019).

6. Yao, L., Bestwick, C. S., Bestwick, L., Maffulli, N. \& Aspden, R. M. Phenotypic drift in human tenocyte culture. Tissue Eng. 12, 1843-1849 (2006).

7. Havis, E. et al. Transcriptomic analysis of mouse limb tendon cells during development. Development 141, 3683-3696 (2014).

$8 . \quad$ Plow, E. F., Haas, T. A., Zhang, L., Loftus, J. \& Smith, J. W. Ligand binding to integrins. J. Biol. Chem. 275, 21785-21788 (2000).

9. Hulsman, M. et al. Analysis of high-throughput screening reveals the effect of surface topographies on cellular morphology. Acta Biomater. 15, 29-38 (2015).

10. Downing, T. L. et al. Biophysical regulation of epigenetic state and cell reprogramming. Nat. Mater. 12, 1154-1162 (2013).

11. Govaert, E. et al. Extracting histones for the specific purpose of label-free MS. Proteomics 16, 2937-2944 (2016).

12. Mitra, A. et al. Cell geometry dictates TNF $\alpha$-induced genome response. Proc. Natl. Acad. Sci. 114, E3882-E3891 (2017).

13. Jain, N. \& Vogel, V. Spatial confinement downsizes the inflammatory response of macrophages. Nat. Mater. (2018). doi:10.1038/s41563-018-0190-6

14. Iwasaki, H., Eguchi, S., Ueno, H., Marumo, F. \& Hirata, Y. Mechanical stretch stimulates growth of vascular smooth muscle cells via epidermal growth factor receptor. Am. J. Physiol. Heart Circ. Physiol. 278, H521-9 (2000). 


\section{Chapter II}

\section{Shaping Cell Fate: Influence of}

\section{Topographical Substratum Properties on Embryonic Stem Cells}

$\underline{\text { Sarita Kumari }}^{1,}, \underline{\text { Steven Vermeulen }}{ }^{2,}$, Ben van der Veer ${ }^{2}$, Aurélie Carlier ${ }^{2}$, Jan de Boer $^{2}$ and Deepa Subramanyam ${ }^{1,4}$

${ }^{1}$ National Centre for Cell Science, SP Pune University, Ganeshkhind Road, Pune -411007, India

${ }^{2}$ Laboratory for Cell Biology-Inspired Tissue Engineering, MERLN Institute, University of Maastricht, Maastricht, The Netherlands.

* These authors contributed equally.

Published in: Tissue Engineering: part B (2018) 


\section{Abstract}

Development of multicellular organisms is a highly orchestrated process, with cells responding to factors and features present in the extracellular milieu. Changes in the surrounding environment help decide the fate of cells at various stages of development. This review highlights recent research that details the effects of mechanical properties of the surrounding environment and extracellular matrix and the underlying molecular mechanisms that regulate the behaviour of embryonic stem cells. Here, we review the role of mechanical properties during embryogenesis and discuss the effect of engineered micro-topographies on embryonic stem cell pluripotency. 


\section{Introduction}

Embryonic stem cells (ESCs) are derived from the preimplantation embryo at the blastocyst stage. These cells possess two valuable properties, namely, they can self-renew indefinitely, and they have the potential to differentiate into cell types belonging to the three germ layers, ${ }^{1}$ ultimately contributing to the entire embryo. ESCs are controlled by a diverse array of signals, including intrinsic cues such as transcription factor networks, ${ }^{2}$ epigenetic modifications, ${ }^{3-6}$ and small RNAs, ${ }^{2-8}$ to name a few. Additionally, extrinsic cues, such as signals originating from the microenvironment and the underlying substratum, also regulate their behavior by affecting several cellular processes, such as cell motility, cell shape, survival, and differentiation..,10

Previous studies have shown that stem cells respond to distinct properties of the extracellular matrix (ECM) that range from mechanical properties, such as stiffness, ${ }^{11}$ adhesiveness, ${ }^{12}$ geometric patterning, ${ }^{13,14}$ and topography, ${ }^{15}$ to biochemical properties involving changes in the composition, ${ }^{16}$ which all, in turn, affect the behavior of the cell. For an overview of the importance of the ECM and the underlying substratum in the context of mesenchymal stem cells, we refer the reader to a number of excellent articles and reviews. ${ }^{17-20}$

These extrinsic cues may also be harnessed to direct and control stem cell fate in the context of tissue engineering and regenerative medicine, in an attempt to deliver patient-specific cell therapies. However, we are still gaining knowledge about how in vitro culture conditions can better mimic the in vivo environment in which stem cells live. This review focuses on how properties of the ECM affect the pluripotency of ESCs while attempting to also draw correlates to the intrinsic behavior of cells residing within the developing mammalian embryo. In the following sections, we highlight examples detailing the role of the ECM in the developing mammalian embryo, the effect of mechanical stimuli that mimic the external substratum through topological cues on pluripotent stem cells, and the underlying molecular mechanism.

\section{Embryonic Development and the Role of the ECM}

The ECM is a dynamic structural component of all tissues, whose composition and constituents change through development, providing the functional cellular environment for the developing embryo. A number of studies illustrate the involvement of the ECM during early vertebrate development, and this has been particularly well characterized in the context of the chick embryo. ${ }^{21-25}$ While it is not possible to present all the instances of ECM involvement in early development, we highlight below the role of a few individual components of the ECM.

During early vertebrate embryo development (chick and mouse), fibronectin (FN) may be assembled in either a paracrine or autocrine manner. ${ }^{26} \mathrm{FN}$, however, remains essential, as mouse embryos null for Fn1, present a shortened anterior-posterior axis, cardiovascular defects, and a general deficit in mesoderm, including impaired somite and notochord formation. ${ }^{27,28}$ The expression and localization of $\mathrm{FN}$ in mouse blastocysts are known to be regulated in response 
to specific growth factors, such as insulin-like growth factor 1 (IGF-1), to enhance their attachment to endometrial cells in vitro. ${ }^{29}$

Other ECM components such as laminin are also expressed early during mammalian development, ${ }^{30,31}$ with laminin b1 and b2 (LAMB1, LAMB2) chains expressed widely at different embryonic stages of human development. ${ }^{32}$ Furthermore, the laminin expression pattern was found to undergo major changes during the differentiation of human ESCs (hESCs), further supporting the idea that the ECM may be both instructive as well as responsive. ${ }^{33}$ Perlecan, a five-domain heparin sulfate proteoglycan is expressed through different stages of human embryogenesis. In particular, its expression is detected at stages where an epithelial-tomesenchymal transition is known to occur, such as during human gut development beginning at gestational week $8 .{ }^{34}$

In addition to the composition of the ECM, the physical properties of the substrate have also been shown to affect early embryonic development. Preimplantation mouse embryos cultured on softer surfaces such as collagen gels (stiffness of $1 \mathrm{kPa}$ ) that mimicked the in vivo uterine environment, developed faster and better from the two-cell stage to the blastocyst, with a significantly higher rate of zona hatching, compared with embryos cultured on stiffer substrates such as polystyrene dishes (stiffness of $1 \mathrm{GPa}$ ). In addition, embryos cultured on softer substrates developed better when transferred to recipient female mice, indicating that the physical properties of the preimplantation environment deeply affected development. ${ }^{35}$ Preimplantation embryos were also sensitive to shear stress, resulting in an induction of phosphorylated MAPK8/9.36 Mimicking of the fallopian tube in vivo environment in terms of shear stress in an in vitro culture system improved the development of embryos to the blastocyst stage, indicating that embryos are responsive to mechanical stimuli. ${ }^{37}$ Thus, the influence of the external environment in terms of chemical composition and physical parameters is far-reaching in terms of early developmental decisions.

\section{Patterned Surfaces as In Vitro Mechanical Niche Models}

In this section, we emphasize the importance of mechanical cues, such as different surface architectures, during embryonic development, and explore how this inherent property can be harnessed for ESCs for fundamental research and tissue engineering purposes. Topographies can be viewed as structures originating from a flat surface and ranging in the nano $(1-1000 \mathrm{~nm})$ or microtopographical $(1-10 \mathrm{~mm}$ ) range, with most research performed on mesenchymal stem cells (MSCs). Disordered nanoscale pits can stimulate the differentiation toward the osteogenic lineage, independent of the classical osteogenic supplement, dexamethasone. ${ }^{38}$ On microtopographies, our group found improved osteogenesis of MSCs. ${ }^{39}$ Also, MSC differentiation down the adipogenic ${ }^{40}$ and chondrogenic ${ }^{41}$ lineages have been previously demonstrated. 
ESC self-renewal can be influenced by combining nanoroughness with geometric shapes. In this study, smooth surfaces $(1 \mathrm{~nm})$ support stemness, whereas nanorough surfaces (70 and $150 \mathrm{~nm}$ ) resulted in a loss of pluripotency. ${ }^{42}$ Furthermore, E-Cadherin (CDH1) presence was maintained on the smooth surface, a crucial regulator for ESC self-maintenance. ${ }^{43}$ In a similar study, flat and nanorough surfaces outperformed microroughness when assessing self-renewal of ESCs. ${ }^{44}$ Here, the flat and nanopatterned surfaces led to reduced ESC adhesion and spreading, suppressed FAK and downstream ERK signaling, improving ESC self-renewal. ${ }^{45}$

In another study, microroughness and nanoroughness were combined (919- $22 \mathrm{~nm})$ and compared against nanoroughness alone $(68-30 \mathrm{~nm})$, a smooth surface, and against a feeder layer. Here, it was observed that in LIF media, the combined approach of utilizing microroughness and nanoroughness promoted formation, homogeneity, and longterm selfrenewal of OCT4-positive colonies.46 Ordered topographical features such as hexagonal (HEX) and honeycomb (HNY) pillars of 50-80 nm with a diameter of 30-40 nm fabricated in polystyrene with variable spacing, were used to study the effects of topography on ESC selfrenewal. Growth on the HEX and HNY pillars was sufficient to maintain OCT4 expression and a higher proliferation rate, compared with flat surfaces without FGF-2 supplementation. ${ }^{47}$

In another approach, silica colloidal crystals between 120 and $600 \mathrm{~nm}$ in diameter were able to improve both ESC self-maintenance and colony formation. ${ }^{48}$ To assess if microtopographies can also guide self-maintenance of pluripotent cells, we used the TopoChip platform, containing 2176 unique topographical features at a constant $10 \mathrm{~mm}$ height profile. ${ }^{49}$ Through machine learning approaches, we observed that a small feature size was a significant determinant for OCT4 expression in induced pluripotent stem cells (iPSCs), which also correlated with higher proliferation.

The BioSurface Structure Array (BSSA) is another microtopographical screening platform with square and round pillars on which 16 different combinations of lateral and gap dimensions were made. Together with a variation in height $(0.6,1.6$, and $2.4 \mathrm{~mm})$, a total of 504 topographical features were used to screen cellular responses, and it was found that ESC colony numbers increased with a decrease in pillar size. ${ }^{50}$ The previous examples highlight the strength of highthroughput screening platforms, where through machine learning algorithms, the most optimal surface architecture can be found for either ESC phenotypic maintenance or differentiation. Figure 1 illustrates the diversity of the cellular response of MSCs and ESCs on the TopoChip platform (unpublished data). 
A

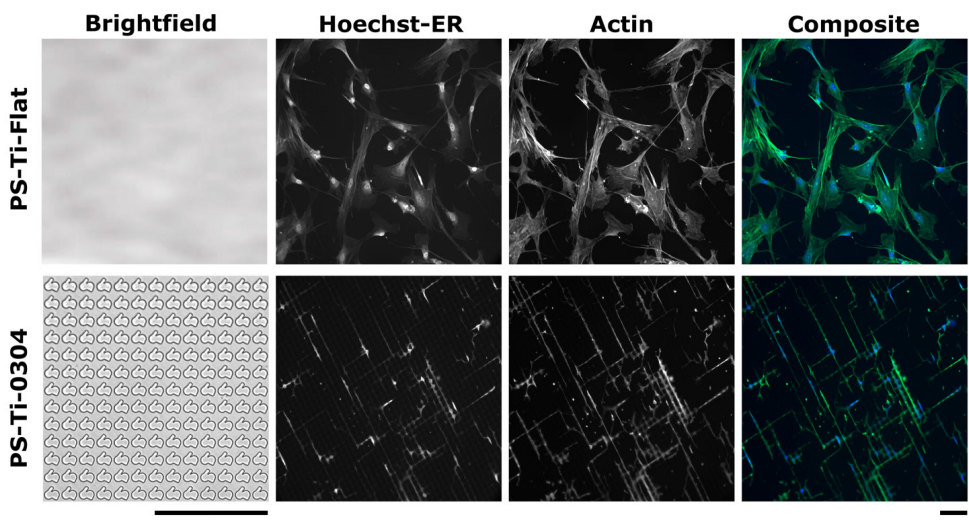

B

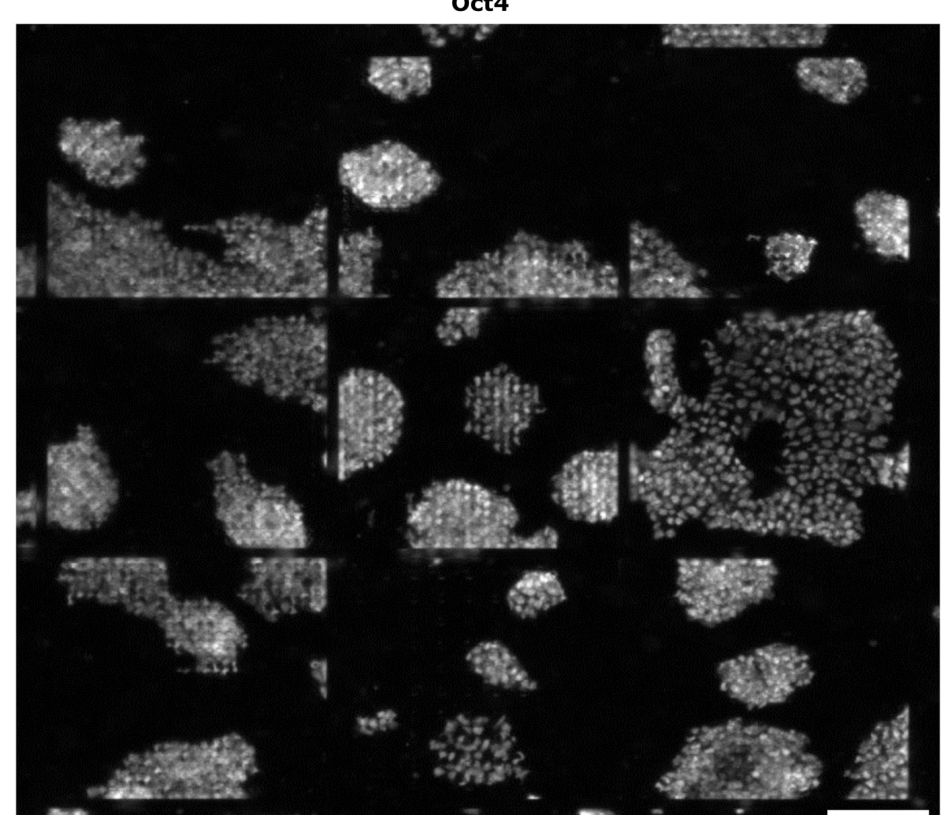

Fig. 1. High-throughput screening tools can identify most optimal substratum properties for either ESC self-renewal or lineage differentiation. (A) Example of a strong morphological adaption of hMSCs cultured on microtopographies. Both the nucleus and cellular morphology are deformed by the topographies (PS-Ti-1018). Costaining for the nucleus [Hoechst], ER [Concanavalin A], and actin [Phalloidin]). (B) Subselection of the TopoChip platform after fixation and OCT4 staining on a 2-day ESC culture. Each topographical unit consists of unique microtopographical features of $10 \mu \mathrm{m}$ height in an area of $290 \mathrm{x} 290$ $\mu \mathrm{m}$ and separated by walls of $50 \mu \mathrm{m}$. Scale bar $=100 \mu \mathrm{m}$. 
As mentioned before, most research aimed at establishing lineage differentiation utilizing topographical features has been performed with MSCs. Still, the knowledge gained from these studies can be useful for protocols utilizing stromal progenitor cells derived from pluripotent cells, ${ }^{51,52}$ to treat, for example, cartilage and bone defects. Besides direct clinical applications, MSCs can also be used as an autologous cell source for supporting ESC self-renewal as a feeder layer. ${ }^{53}$ To allow ESC differentiation toward MSCs without the need for chemical induction, square-patterned nanopits were able to upregulate mesodermal marker expression and downregulate pluripotent, ectodermic, and endodermal markers. ${ }^{54}$ Of note, these surfaces were the same topographical structures used to induce osteogenic differentiation of MSCs without the need for dexamethasone,38 offering a promise for ESC differentiation for bone tissue engineering applications using only topographical cues. A large body of research involving the differentiation of pluripotent cells through topographies focuses on the generation of cells of the neuronal lineage. hESCs seeded on gelatin-coated nanoscale groove patterns (spacing $=350$ $\mathrm{nm}$; height $=500 \mathrm{~nm}$ ) induced the expression of NEUROD1 among other neurogenic markers. ${ }^{55}$ A similar observation was made when iPSCs were seeded on Matrigel-coated nanoscale grooved patterns (spacing $=350 \mathrm{~nm}$; height $=300 \mathrm{~nm}$ ) fabricated on PDMS. Expression of neurogenic markers, such as TUBB3, NEUROD1, and NEUROG1, was higher on these nanotopographical dimensions compared with flat, 2 or $5 \mathrm{~mm}$ spacing. ${ }^{55,56}$ In another study, Matrigel-coated polystyrene gratings of $2 \mathrm{~mm}$ width were able to efficiently induce neural differentiation in the absence of neurotrophic inducing chemicals at later stages of the culture. ${ }^{42}$

Besides improving differentiation efficiency, the induction of neuronal subtypes can be achieved using isotropic surfaces. ${ }^{57}$ Studies on $250 \mathrm{~nm}$ grooved topographies linked ESC actomyosin contractility with neuronal marker expression. ${ }^{58}$ It is interesting to mention that nanotopographical grooves of $350 \mathrm{~nm}$ have also been previously used to induce MSC differentiation toward the neurogenic lineage. ${ }^{59}$ To summarize, these studies reveal that both micro- and nanotopographical cues can play a crucial role in formulating more efficient and xenofree protocols for ESC differentiation toward the neurogenic lineages.

To investigate endoderm differentiation, tall nanopillars with different aspect ratios were used, through which stiffness parameters could be altered. It was found that tall pillars, mimicking a softer substrate, allowed a higher efficiency of endoderm differentiation after chemical stimulation. ${ }^{60}$ In another approach, $4.5 \mathrm{~nm}$ diameter gold particles were combined with different chemistries to study ESC behavior, with all nanoparticle films inducing the expression of FOXA2, a marker for early endoderm commitment. Furthermore, this study provided evidence that ESCs can sense topographical cues smaller than $5 \mathrm{~nm} .{ }^{61}$ Although soluble growth factors are still required in these protocols, these studies indicate that biophysical cues can influence the endodermal differentiation of pluripotent stem cells.

Although this review highlights the influence of topographical cues on stem cells, research investigating differences in their response to topography of the two majorly studied pluripotent cell types (ESCs and iPSCs), is relatively limited. Research does indicate distinct gene expression profiles between iPSCs and ESCs, ${ }^{62}$ which can lead to differences in differentiation efficiencies. ${ }^{63}$ 
Furthermore, the cellular origin of iPSCs influences lineage differentiation propensity. ${ }^{64}$ Therefore, the possibility arises that iPSC and ESC cell lines may have a variable response to topographical cues. In this context, it has been shown that different ESC lines respond slightly differently to microtopographical cues in terms of self-renewal and differentiation. ${ }^{50}$ Furthermore, the use of two different ESC lines show differences in marker expression when differentiated toward neuronal lineages on different topographical structures. ${ }^{57}$ Recently, Abagnale et al. showed that submicrometer groove-ridge structures can modulate the shape of iPSC colonies, regulate cell polarity, and guide the orientation of actin fibers. ${ }^{65}$

Altogether, these studies indicate that both ESCs and iPSCs respond to topographical cues, yet not necessarily in a completely similar manner. Future research, where the influence of topographical cues on both ESCs and iPSCs in the same experimental setting is investigated, might explore this observation more deeply.

\section{Molecular Mechanism of Sensing the Underlying Substratum}

The previous sections demonstrate the influence of the ECM and topographical cues on ESC behavior and fate commitment. However, the exact molecular mechanisms that underlie these phenomena are unknown. In the following sections, we aim to summarize current knowledge and identify open questions.

\section{Integrins and Downstream Kinases}

Several molecules sense changes in the underlying substratum and help convert this into a definitive transcriptional output that regulates cell fate. Integrins are one of the important substrate- and ECM-sensing proteins. ${ }^{66,67}$ Integrins are fundamental components of focal adhesions. These are heterodimeric receptors made up of one b subunit with one a subunit, clustered in different combinations in response to specific ECM proteins. ${ }^{68}$ Integrins play a major role in mechanosensing and sensing differences in ECM composition. ${ }^{69,70}$ Hayashi et al. demonstrate that the expression of integrins is dependent on the composition of the ECM. They further demonstrate that the overexpression of integrin subunits (specifically those subunits that are induced in ESCs upon culturing them on Type I collagen) results in the differentiation of mESCs, whereas inactivation of specific integrin subunits helps promote mESC self-renewal. ${ }^{69}$ Furthermore, in the context of mesenchymal stem cells, it is known that the expression and clustering of integrin receptors changes in response to the stiffness of the underlying matrix. ${ }^{71}$

Specific integrins were shown to be activated in response to different ranges of stiffness. In response to medium stiffness $(10.2 \mathrm{kPa})$, MSCs differentiated into myocytes through $\beta 3$ receptor-mediated signaling, whereas MSCs switched to ITGA2 in response to a stiffer matrix $(40.7 \mathrm{kPa})$ resulting in differentiation down the osteocyte lineage. This integrin switching in response to substrate stiffness also resulted in a change in the size of focal adhesions. ${ }^{72}$ 
Interactions between integrins and the ECM result in a number of downstream signaling events, some of which involve the SRC family kinases. ${ }^{73}$ Integrin-mediated focal adhesion kinase (FAK)SRC signaling regulates cell adhesion dynamics by regulating the activity of the small GTPase, RHO. RHO functions as a major target molecule involved in mESC differentiation by activating downstream kinases such as RHO kinase (ROCK), resulting in cell spreading. ${ }^{74}$ Significantly, the presence of a pharmacological inhibitor of ROCK not only blocked mESC spreading and differentiation and promoted colony formation, but also resulted in maintenance of OCT4 and NANOG expression even in the absence of LIF, although mESC numbers were lower than when cultured in the presence of LIF. ${ }^{75}$ The function of RHO and ROCK in hESCs appears to depend heavily on the cellular context. Dissociated hESCs were susceptible to apoptosis due to actomyosin contractility, and this could be blocked in the presence of a ROCK inhibitor. ${ }^{76,77}$ Apoptosis was driven in these cells under conditions where RHO activity was high compared with RAC. ${ }^{77}$ However, contrary to this, in intact hESC colonies, RHO was essential for the survival and propagation of hESCs. ${ }^{78}$

The role of FAK in the maintenance of stem cell survival and pluripotency has been shown to vary between human and mouse ESCs. In fact, even within hESCs, the role of FAK remains controversial. Vitillo et al. report that integrin associated FAK is active in hESCs, and that this signaling is important for protecting cells from apoptosis upon detachment. Furthermore, this signaling is required for the maintenance of the pluripotent state. ${ }^{79}$ However, contrary to this, Villa-Diaz et al. report that FAK remains inactive in hESCs due to the expression of ITGA6. During the differentiation of hESCs, the levels of ITGA6 decrease and ITGB1 gets activated, resulting in an activation of $\mathrm{FAK}$, and differentiation of hESCs, ${ }^{80}$ whereas specific combinations of integrins have been shown to maintain stemness in mESCs, even in the absence of LIF. ${ }^{81}$ In mouse ESCs, FAK remains inactive and the expression of integrins is shown to undergo a switch in response to differentiation. ${ }^{69}$

Some reports also show that FAK/SRC interacting partners may play an important role in integrin signaling. Knockout of one such interactor, Paxillin (Pxn), in ESCs shows delayed cell spreading, reduced FAK phosphorylation, and differentiation. ${ }^{82}$ Interestingly, proteomic analysis comparing ESCs grown in serum with cells cultured in $2 \mathrm{i}$ (a defined medium containing inhibitors against GSK3b and MEK), showed higher levels of integrins and actin-binding proteins, such as talin, vinculin, and filamin in cells maintained in serum. ${ }^{83}$ It is well known that serum contains many ECM components with prime differentiation, indicating that serum induces changes in the molecular ECM-sensing mechanism. Thus, the sensing of the ECM through integrins, coupled with the activation of specific downstream kinases and actin regulation may play a key role in maintaining specific cell fate (Figure 2).

In addition to integrins, mechanosensitive ion channels are also emerging as novel players in sensing mechanical force and tension. ${ }^{84,85} \mathrm{~A}$ specific family of eukaryotic mechanosensitive channels, PIEZO, appears to play a role in maintaining homeostatic cell numbers in epithelia. ${ }^{86}$ Interestingly, PIEZO1 activity can be triggered by traction forces and played an important role in the differentiation of neural stem cells to either an astrocytic or a neuronal lineage. ${ }^{87}$ Thus, 
sensing of the external environment through the abovementioned molecules and mechanisms results in signal transduction within the stem cell for regulating cell fate choices.

\title{
Pluripotency
}

\section{Pluripotent Stem cell}

Differentiated cell
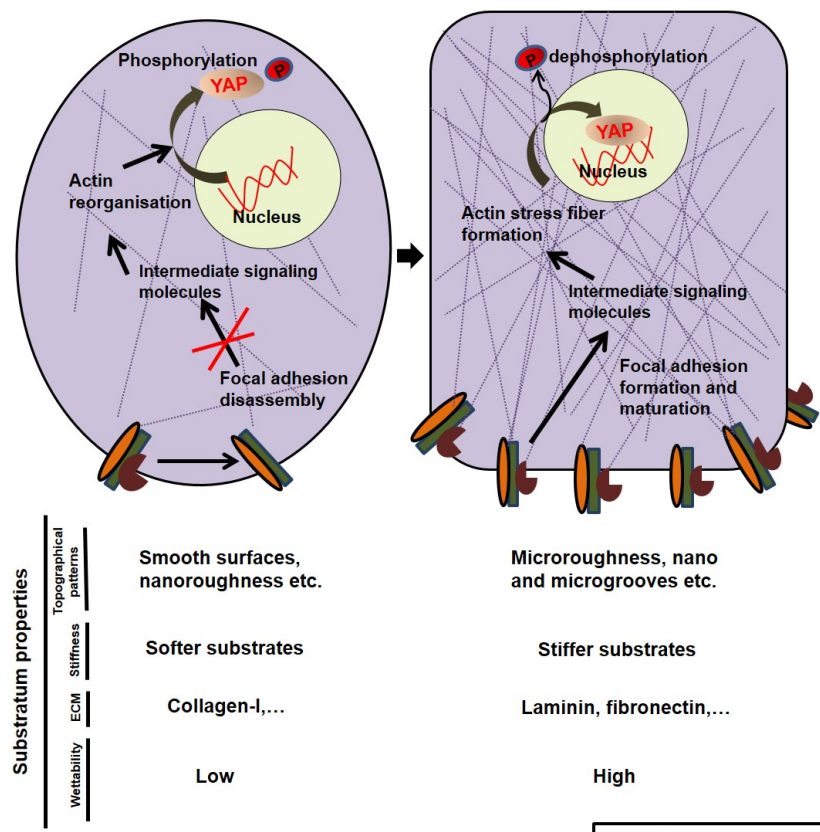

\begin{abstract}
Microroughness, nano
\end{abstract} and microgrooves etc.

Stiffer substrates

Laminin, fibronectin,...

High

$\alpha$ integrin subunit

$\beta$ integrin subunit

Mature focal adhesion

Actin stress fibers

Fig. 2. Molecular sensing of substratum properties by stem cells. Stem cells respond to the underlying substratum properties such as topography, stiffness, ECM composition, and wettability by changing the expression and engagement of integrins in a contextdependent manner. A change in the integrin composition affects the assembly and disassembly of focal adhesions and the recruitment of intermediate signaling molecules. These signals ultimately connect either directly or indirectly with the actin cytoskeleton and regulate the translocation of YAP/TAZ from the cytoplasm to the nucleus, resulting in a change in pluripotency. 


\section{Actin Dynamics and the Hippo Pathway}

The mechanism of signal transduction within the stem cell requires a connection between the ECM and the nucleus. This is mediated by a complex set of interactions involving the integrins, actin cytoskeleton, actin-binding proteins, and other proteins that play a critical role in regulating downstream signaling. As mentioned above, the underlying substrate properties, such as stiffness and surface topography, lead to the activation of several kinases such as the SRC kinase, which further results in the activation of RHO. This further regulates the formation of F-actin, thus promoting actomyosin contractility, and leading to the translocation of YAP to the nucleus (Figure 2). ${ }^{88}$ YAP (Yorkie ortholog) is a part of the Hippo pathway that was first elucidated in Drosophila through genetic mosaic screens for tumor suppressors. ${ }^{89}$ Upstream signals result in the activation of downstream kinases, such as LATS1/2, which is responsible for the phosphorylation of YAP. The phosphorylated form of YAP is sequestered in the cytoplasm and is thus unable to activate downstream transcription. Hypophosphorylated forms of YAP/TAZ (Yorkie orthologs) are able to enter the nucleus and initiate transcription together with TEAD14, which contain DNA-binding domains. ${ }^{89}$

YAP/TAZ has been implicated in playing a major role in sensing mechanical changes. Their phosphorylation status and subsequent shuttling between the nucleus and cytoplasm act to regulate gene expression changes in response to changes in substratum properties, such as substrate stiffness, topography, and cyclic stretching. ${ }^{88}$ The Hippo pathway was shown to function as a barrier during the reprogramming of human somatic cells to induced pluripotency, as the knockdown of LATS2 enhanced the reprogramming efficiency. ${ }^{90}$ In support of this, overexpression of YAP also promoted the conversion of hESCs to a naive state. ${ }^{91}$ Conversely, in mESCs, loss of YAP could continue to support the pluripotent state, whereas YAP functioning was required for proper differentiation. ${ }^{22}$

Actin and RHO-processing factors directly or indirectly regulate YAP/TAZ translocation by affecting the stress fiber stability in response to several physical and mechanical clues ${ }^{8}{ }^{8}$ In intact hESCs, Rho and its activator, AKAP-Lbc, maintain the nuclear function of YAP/TAZ by regulating actin filament organization. ${ }^{78}$ Interestingly, a different study demonstrated that the knockdown of specific kinases, including TESK1 or LIMK2, promoted the reprogramming of somatic cells to induced pluripotency by decreasing cofilin phosphorylation and disrupting actin filament structures. ${ }^{93}$

Overall, the dynamic shuttling, transcriptional activity, and in some cases the expression of YAP/TAZ are tightly linked to matrix stiffness. ${ }^{88}$ Within developing embryos as well, it has been shown that tensile forces generated by the actomyosin network are responsible for the positioning of cells and embryo compaction, while also regulating the localization of YAP.94,95 YAP has also been shown to be involved in the maintenance of both naive as well as primed pluripotency, and for the regulation of ESC differentiation. ${ }^{78,91,92,96-98}$ 
In addition to the involvement of the Hippo pathway, the nucleus is also physically linked to the ECM due to the continuous network of intracellular and extracellular fibers. As such, topographical cues can directly affect the nucleus mechanically, resulting in altered gene expression through epigenetic modifications or chromatin and laminar reorganization. ${ }^{98}$ The nuclear morphology of mesenchymal stem cells was more sensitive than differentiated cells to the architecture of nanofibrous scaffolds on which they were seeded. Application of tension to the scaffolds further caused changes in nuclear morphology of stem cells that were mediated by the actin cytoskeleton. ${ }^{99}$ Kulangara et al. showed that mesenchymal stem cells cultured on 350 $\mathrm{nm}$ gratings showed a reduction in levels of LMNA and RB and a reduction in cell proliferation. ${ }^{100}$ Changes in gene expression patterns were also observed in cells due to seeding on substrates with geometric patterning through actomyosin contractility. ${ }^{101}$ Despite this wealth of literature, the precise mechanism connecting the ECM to the nucleus through the actin cytoskeleton, and alterations in gene expression still remains to be worked out in pluripotent stem cells.

\section{The Influence of Topographical Cues on Integrin and Hippo Signaling}

Cells are cultured in a multifactorial environment, which makes it difficult to fully assess the influence that topographical cues exert on stem cell fate. Utilizing materials with different stiffness, chemistry, surface coatings, and culture media can affect cell culture outcomes and potentially mask topographical influences. This is further complicated by the interconnectedness of topographies with wettability and even stiffness, ${ }^{102}$ both known to be major influencers in stem cell self-renewal and differentiation. ${ }^{103-105}$ Nevertheless, the influence of topographies can be independent of wettability as shown in the studies of Jaggy et al. and Jeon et al.45,46

Despite this environmental complexity, trends can be seen across multiple studies, for example, comparing different material roughness levels on stem cell maintenance shows that a smooth surface performs equal or better then nanoscale levels, ${ }^{42,44-46}$ and outperforms microscale roughness. ${ }^{44}$ However, when combining both micro and nanoroughness, stem cell self-renewal was promoted, compared with a flat and nanorough surface. ${ }^{46}$ On the nanoscale level, promotion of stem cell pluripotency is possible through the use of repetitive crystal structures ${ }^{48}$ or pillars, ${ }^{47}$ illustrating that besides size, other feature parameters such as symmetrical patterns can have an important effect on cell fate. Microtopographical cues can support a pluripotent phenotype, yet this is very dependent on feature parameters. In the study of Markert $e t$ al., higher features are supportive for self-renewal among the distance of the patterns, ${ }^{50}$ whereas in the study of Reimer et al., a lower pattern density is associated with pluripotency. ${ }^{49}$ The use of grooves or ridges in both micro and nanoscale dimensions are generally associated with promoting differentiation of pluripotent cells. ${ }^{55-58,65,106}$

In the previous sections, we emphasized the role of integrin signaling and specific topographical cues on ESC and iPSC cell fate. Activation of integrin signaling is associated with loss of pluripotency, ${ }^{69}$ and topographies could influence this signaling through the focal adhesion kinase 22 
(FAK). ${ }^{107}$ Cells grown on surfaces with low roughness promote pluripotency, show less focal adhesions, and reduced aligned actin stress fibers (Figure 2). ${ }^{47}$ Furthermore, increased phosphorylation of FAK, vinculin, and increased actomyosin contractility is associated with the induction of differentiation through the activation of its downstream signaling pathways. ${ }^{45,46,108-}$ 110 Topographical cues can also alter the spatial arrangement of FAKs and negatively influence pluripotency. ${ }^{42}$ Furthermore, spreading of pluripotent cells is associated with increased integrinmatrix interactions and loss of self-renewal. ${ }^{69,111,112}$ The wettability of the substrate is associated with cell spreading and increased FAK formation, ${ }^{113,114}$ making this an important parameter in cell culture studies. Considering this, micro- and nanotopographical surfaces are known to influence adhesion characteristics of pluripotent stem cells. ${ }^{42,45,48,50,115}$

FAK signaling can regulate MSC differentiation as well, and can guide differentiation towards the osteogenic lineage through the activation of canonical and noncanonical signaling pathways mediated by cytoskeleton reorganization, ${ }^{108-110,116}$ whereas a decrease in FAK presence is associated with a higher potential to differentiate toward the adipogenic lineage. ${ }^{107,117}$ Also in this context, differentiation can be mediated by nanotopographical cues. ${ }^{107,118}$

We previously elaborated on the role of actin-myosin dynamics and Hippo signaling in ESC pluripotency and differentiation. The downstream transcriptional co-activators, YAP and TAZ, are mechanosensitive barometers of the cell118,119 and are affected in MSCs by both surface stiffness and forced spreading, ${ }^{88}$ influencing their differentiation capability. ${ }^{120}$ The effect of these substrates and topographical cues on YAP and TAZ in ESCs and iPSCs have been less investigated. Nevertheless, topographical features are capable of enhancing the osteogenic differentiation of MSCs by increasing cell attachment, actin rearrangement, and promoting the activation of the YAP/TAZ signaling pathway. ${ }^{121}$ Specific nanopatterns that are optimal for inducing osteogenic differentiation showed enhanced YAP activity when MSCs were cultured on such surfaces. ${ }^{122}$ Nanotopographical features were also shown to promote the differentiation of hESCs and iPSCs into pancreatic endocrine cells ${ }^{123}$ and neuronal cells, ${ }^{124}$ through the regulation of the nucleocytoplasmic localization of YAP/TAZ.

In recent work by Abagnale et al., where nanoscale ridges support BMP4-induced differentiation of iPSCs, the spatial distribution of TAZ was colocalized with actin filaments and cell material adhesion sites when culturing iPSCs on nanogrooves. ${ }^{65}$ These studies support the notion that YAP/TAZ pathways can play a role in transmitting topography-induced mechanical cues for pluripotent stem cells.

\section{Relevance to Tissue Engineering and Regenerative Medicine}

Human tissues are made up of many types of cells, which in turn interact with a variety of ECM proteins. The organization of the ECM in each tissue and organ varies from nano to microscale proteins with different feature sizes. Micro- and nanoscale proteins from the ECM transmit chemical and physical cues to cells controlling various behaviors, including their adhesion, 
proliferation, migration, and differentiation. Therefore, topography-guided approaches that attempt to mimic the natural ECM has become a field of topical interest, with the aim of optimizing the generation of 3D artificial tissue grafts from stem cells. ${ }^{125-128}$

Substrates with topographical features can be beneficial in the field of tissue engineering, with noteworthy examples, including improving tissue-implant interaction, ${ }^{129,130}$ generation of cardiomyocytes from iPSCs, ${ }^{131}$ improving the retention time of transplanted cells and their integration into the host tissue, ${ }^{132,133}$ generation of mature muscle patches, ${ }^{134}$ and improving the process of skin regeneration. ${ }^{35}$ Altogether, these reports strengthen the notion that topographical cues can help direct cell fate down a specific lineage and that patterned surfaces can be employed efficiently in the field of regenerative medicine.

\section{Open Questions and Future Directions}

During development, ESCs do not develop in an independent and isolated fashion. Instead, they respond to and interact with various biological, chemical, and physical cues. To further our fundamental understanding of embryonic development (and its relation to particular pathologies), as well as design improved in vitro culture methods to advance the field of regenerative medicine, more in-depth studies are needed to unravel the role of the ECM in ESC behavior. It is important to note that due to the complex interplay between ESCs and ECM and the large number of ways in which the ECM can be modified, combinatorial, high-throughput screening approaches, typically used for drug discovery in the pharmaceutical industry, will offer novel opportunities (defined as "materiomics"). ${ }^{136}$ Recently, with the emergence of micro and nanotechnologies, novel platforms have been developed to systematically probe the cellsubstrate interface using cell size limiting adhesive islands from fibronectin, ${ }^{14}$ patterned surface pits, ${ }^{15}$ patterned cell shapes, ${ }^{101}$ or varying matrix elasticity or stiffness. ${ }^{11,137}$ Taking this a step further, others have developed platforms to independently and simultaneously manipulate two cell-ECM interfacial properties, such as cell size and cell shape, ${ }^{13}$ or ECM stiffness and pore size, ${ }^{138}$ and investigate their influence on cell behavior. Moreover, to systematically study large numbers of surfaces, the platforms mentioned above are being miniaturized, enabling highthroughput screening of surface topographies ${ }^{50,139}$ or chemistry. ${ }^{140,141}$

Altogether, with the development of combinatorial high-throughput techniques, developing advanced data mining techniques and system biological models are essential tools to identify patterns in the data, generate novel hypotheses, and to perform in silico experiments for a wide range of different conditions. Several examples in the literature exist such as (dynamic) models of gene regulatory networks that allow exploring reprogramming strategies, ${ }^{142-144}$ computational models of autocrine and paracrine signaling to determine the soluble factors essential for mESC survival in vitro, ${ }^{145}$ and computational fluid dynamics models to optimize the culture conditions to promote efficient cardiogenesis of hydrogel encapsulated ESCs in a rotating bioreactor. ${ }^{146}$ For more detailed information on computational modeling, we refer the reader to the following excellent reviews. ${ }^{147-149}$ 
In summary, although it is clearly recognized that the ECM plays an important role during embryonic development, several outstanding questions remain. What are the molecular mechanisms that underlie ECM sensing, and how do they interact? How do these (mechanobiological) pathways change during embryonic development and lineage specification? Are these pathways conserved among different species, and if so, what can we learn from this? Finally, how can we harness these mechanobiological cues to advance current scientific and clinical models of embryonic development?

\section{Conclusions}

A key component to successfully translating regenerative therapies from the bench to the bedside is an in-depth understanding of early embryonic development. In this review, we have detailed how mechanical properties of the ECM affect the pluripotency of the ESCs, in addition to a myriad of well-studied soluble factors, including growth factors and cytokines. It appears that the effect of mechanical cues (such as topography) that replicate the conditions within the developing embryo, favor ESC maintenance. Interestingly, although the influence of mechanical cues is well recognized, the exact underlying molecular mechanisms are poorly understood in the context of stem cells. However, as the field progresses through the development of new tools to study the cell-material interface, details of particular signal transduction pathways will emerge. As such, we believe that interdisciplinary collaborations of material scientists and developmental biologists will have great potential to illuminate the fundamental questions on ESC behavior and improve existing regenerative therapies.

\section{Acknowledgments}

The authors regret their inability to cite all the articles in the field due to space constraints. S.K. is a recipient of a Senior Research Fellowship from the Department of Biotechnology, India. J.d.B., A.C., B.V., and S.V. acknowledge the financial support of the Dutch province of Limburg. A.C. gratefully acknowledges the Dutch Science Foundation (NWO) for a VENI grant (15075), S.V. acknowledges the financial support of the European Union's Horizon 2020 Program (H2020- MSCA-ITN-2015; Grant agreement 676338). The authors thank members of the Subramanyam Laboratory and the de Boer group for critical comments.

\section{Disclosure Statement}

No competing financial interests.

\section{References}

1. Evans, M.J., and Kaufman, M.H. Establishment in culture of pluripotential cells from mouse embryos. Nature 292, 154, 1981.

2. Boyer, L.A., Lee, T.I., Cole, M.F., et al. Core transcriptional regulatory circuitry in human embryonic stem cells. Cell 122, 947, 2005.

3. Bernstein, B.E., Mikkelsen, T.S., Xie, X., et al. A bivalent chromatin structure marks key developmental genes in embryonic stem cells. Cell 125, 315, 2006. 
4. Boyer, L.A., Mathur, D., and Jaenisch, R. Molecular control of pluripotency. Curr Opin Genet Dev 16, 455, 2006.

5. Boyer, L.A., Plath, K., Zeitlinger, J., et al. Polycomb complexes repress developmental regulators in murine embryonic stem cells. Nature 441, 349, 2006.

6. Lee, T.I., Jenner, R.G., Boyer, L.A., et al. Control of developmental regulators by Polycomb in human embryonic stem cells. Cell 125, 301, 2006.

7. Marson, A., Levine, S.S., Cole, M.F., et al. Connecting microRNA genes to the core transcriptional regulatory circuitry of embryonic stem cells. Cell 134, 521, 2008.

8. Wang, Y., Medvid, R., Melton, C., Jaenisch, R., and Blelloch, R. DGCR8 is essential for microRNA biogenesis and silencing of embryonic stem cell self-renewal. Nat Genet 39, 380, 2007.

9. Eshghi, S., and Schaffer, D.V. Engineering microenvironments to control stem cell fate and function. StemBook [Internet].Cambridge (MA): Harvard Stem Cell Institute, 2008.

10. Gattazzo, F., Urciuolo, A., and Bonaldo, P. Extracellular matrix: a dynamic microenvironment for stem cell niche. Biochim Biophys Acta 1840, 2506, 2014.

11. Engler, A.J., Sen, S., Sweeney, H.L., and Discher, D.E. Matrix elasticity directs stem cell lineage specification. Cell 126, 677, 2006.

12. Murray, P., Prewitz, M., Hopp, I., et al. The self-renewal of mouse embryonic stem cells is regulated by cellsubstratum adhesion and cell spreading. Int J Biochem Cell Biol 45, 2698, 2013.

13. Kilian, K.A., Bugarija, B., Lahn, B.T., and Mrksich, M. Geometric cues for directing the differentiation of mesenchymal stem cells. Proc Natl Acad Sci U S A 107, 4872, 2010.

14. McBeath, R., Pirone, D.M., Nelson, C.M., Bhadriraju, K., and Chen, C.S. Cell shape, cytoskeletal tension, and RhoA regulate stem cell lineage commitment. Dev Cell 6, 483, 2004.

15. Dalby, M.J., Gadegaard, N., Tare, R., et al. The control of human mesenchymal cell differentiation using nanoscale symmetry and disorder. Nat Mater 6, 997, 2007.

16. Wang, H., Luo, X., and Leighton, J. Extracellular matrix and integrins in embryonic stem cell differentiation. Biochem Insights 8, 15, 2015.

17. Guilak, F., Cohen, D.M., Estes, B.T., Gimble, J.M., Liedtke, W., and Chen, C.S. Control of stem cell fate by physical interactions with the extracellular matrix. Cell Stem Cell 5, 17, 2009.

18. Lane, S.W., Williams, D.A., and Watt, F.M. Modulating the stem cell niche for tissue regeneration. Nat Biotechnol 32, 795, 2014.

19. Lin, X., Shi, Y., Cao, Y., and Liu, W. Recent progress in stem cell differentiation directed by material and mechanical cues. Biomed Mater 11, 014109, 2016.

20. Watt, F.M., and Huck, W.T. Role of the extracellular matrix in regulating stem cell fate. Nat Rev Mol Cell Biol 14, 467, 2013.

21. Hamburger, V., and Hamilton, H.L. A series of normal stages in the development of the chick embryo. J Morphol 88, 49, 1951.

22. Zagris, N., Stavridis, V., and Chung, A.E. Appearance and distribution of entactin in the early chick embryo. Differentiation 54, 67, 1993.

23. Ebendal, T. Extracellular matrix fibrils and cell contacts in the chick embryo. Possible roles in orientation of cell migration and axon extension. Cell Tissue Res 175, 439, 1977.

24. Zagris, N. Extracellular matrix in development of the early embryo. Micron 32, 427, 2001.

25. Hurle, J.M., Ros, M.A., Climent, V., and Garcia-Martinez, V. Morphology and significance of programmed cell death in the developing limb bud of the vertebrate embryo. Microsc Res Tech 34, 236, 1996.

26. de Almeida, P.G., Pinheiro, G.G., Nunes, A.M., Goncalves, A.B., and Thorsteinsdottir, S. Fibronectin assembly during early embryo development: a versatile communication system between cells and tissues. Dev Dyn 245, 520, 2016.

27. George, E.L., Georges-Labouesse, E.N., Patel-King, R.S., Rayburn, H., and Hynes, R.O. Defects in mesoderm, neural tube and vascular development in mouse embryos lacking fibronectin. Development 119, 1079, 1993.

28. Georges-Labouesse, E.N., George, E.L., Rayburn, H., and Hynes, R.O. Mesodermal development in mouse embryos mutant for fibronectin. Dev Dyn 207, 145, 1996.

29. Green, C.J., Fraser, S.T., and Day, M.L. Insulin-like growth factor 1 increases apical fibronectin in blastocysts to increase blastocyst attachment to endometrial epithelial cells in vitro. Hum Reprod 30, 284, 2015. 
30. Gersdorff, N., Muller, M., Otto, S., Poschadel, R., Hubner, S., and Miosge, N. Basement membrane composition in the early mouse embryo day 7. Dev Dyn 233, 1140, 2005.

31. Gersdorff, N., Kohfeldt, E., Sasaki, T., Timpl, R., and Miosge, N. Laminin gamma3 chain binds to nidogen and is located in murine basement membranes. J Biol Chem 280, 22146, 2005.

32. Roediger, M., Miosge, N., and Gersdorff, N. Tissue distribution of the laminin beta1 and beta 2 chain during embryonic and fetal human development. J Mol Histol 41, 177, 2010.

33. Pook, M., Teino, I., Kallas, A., Maimets, T., Ingerpuu, S., and Jaks, V. Changes in laminin expression pattern during early differentiation of human embryonic stem cells. PLoS One 10, e0138346, 2015.

34. Roediger, M., Kruegel, J., Miosge, N., and Gersdorff, N. Tissue distribution of perlecan domains III and $\mathrm{V}$ during embryonic and fetal human development. Histol Histopathol 24, 859, 2009.

35. Kolahi, K.S., Donjacour, A., Liu, X., et al. Effect of substrate stiffness on early mouse embryo development. PLoS One 7, e41717, 2012.

36. Xie, Y., Wang, F., Puscheck, E.E., and Rappolee, D.A. Pipetting causes shear stress and elevation of phosphorylated stress-activated protein kinase/jun kinase in preimplantation embryos. Mol Reprod Dev 74, 1287, 2007.

37. Matsuura, K., Hayashi, N., Kuroda, Y., et al. Improved development of mouse and human embryos using a tilting embryo culture system. Reprod Biomed Online 20, 358, 2010.

38. Dalby, M.J., Gadegaard, N., Curtis, A.S., and Oreffo, R.O. Nanotopographical control of human osteoprogenitor differentiation. Curr Stem Cell Res Ther 2, 129, 2007.

39. Hulshof, F.F.B., Papenburg, B., Vasilevich, A., et al. Mining for osteogenic surface topographies: in silico design to in vivo osseo-integration. Biomaterials 137, 49, 2017.

40. Abagnale, G., Steger, M., Nguyen, V.H., et al. Surface topography enhances differentiation of mesenchymal stem cells toward osteogenic and adipogenic lineages. Biomaterials 61, 316, 2015.

41. Gao, L., McBeath, R., and Chen, C.S. Stem cell shape regulates a chondrogenic versus myogenic fate through Rac1 and N-cadherin. Stem Cells 28, 564, 2010.

42. Chen, W., Villa-Diaz, L.G., Sun, Y., et al. Nanotopography influences adhesion, spreading, and selfrenewal of human embryonic stem cells. ACS Nano 6, 4094, 2012.

43. Redmer, T., Diecke, S., Grigoryan, T., Quiroga-Negreira, A., Birchmeier, W., and Besser, D. E-cadherin is crucial for embryonic stem cell pluripotency and can replace OCT4 during somatic cell reprogramming. EMBO Rep 12, 720, 2011.

44. Lyu, Z., Wang, H., Wang, Y., et al. Maintaining the pluripotency of mouse embryonic stem cells on gold nanoparticle layers with nanoscale but not microscale surface roughness. Nanoscale 6, 6959, 2014.

45. Jeon, K., Oh, H.J., Lim, H., et al. Self-renewal of embryonic stem cells through culture on nanopattern polydimethylsiloxane substrate. Biomaterials 33, 5206, 2012.

46. Jaggy, M., Zhang, P., Greiner, A.M., et al. Hierarchical micro-nano surface topography promotes longterm maintenance of undifferentiated mouse embryonic stem cells. Nano Lett 15, 7146, 2015.

47. Kong, Y.P., Tu, C.H., Donovan, P.J., and Yee, A.F. Expression of Oct4 in human embryonic stem cells is dependent on nanotopographical configuration. Acta Biomater 9, 6369, 2013.

48. Ji, L., LaPointe, V.L., Evans, N.D., and Stevens, M.M. Changes in embryonic stem cell colony morphology and early differentiation markers driven by colloidal crystal topographical cues. Eur Cell Mater 23, 135, 2012.

49. Reimer, A., Vasilevich, A., Hulshof, F., et al. Scalable topographies to support proliferation and Oct4 expression by human induced pluripotent stem cells. Sci Rep 6, 18948, 2016.

50. Markert, L.D., Lovmand, J., Foss, M., et al. Identification of distinct topographical surface microstructures favoring either undifferentiated expansion or differentiation of murine embryonic stem cells. Stem Cells Dev 18, 1331, 2009.

51. Brown, S.E., Tong, W., and Krebsbach, P.H. The derivation of mesenchymal stem cells from human embryonic stem cells. Cells Tissues Organs 189, 256, 2009.

52. de Peppo, G.M., Svensson, S., Lenneras, M., et al. Human embryonic mesodermal progenitors highly resemble human mesenchymal stem cells and display high potential for tissue engineering applications. Tissue Eng Part A 16, 2161, 2010.

53. Cheng, L., Hammond, H., Ye, Z., Zhan, X., and Dravid, G. Human adult marrow cells support prolonged expansion of human embryonic stem cells in culture. Stem Cells 21, 131, 2003.

54. Kingham, E., White, K., Gadegaard, N., Dalby, M.J., and Oreffo, R.O. Nanotopographical cues augment mesenchymal differentiation of human embryonic stem cells. Small 9, 2140, 2013. 
55. Lee, M.R., Kwon, K.W., Jung, H., et al. Direct differentiation of human embryonic stem cells into selective neurons on nanoscale ridge/groove pattern arrays. Biomaterials 31, 4360, 2010.

56. Pan, F., Zhang, M., Wu, G., et al. Topographic effect on human induced pluripotent stem cells differentiation toward neuronal lineage. Biomaterials 34, 8131, 2013.

57. Ankam, S., Suryana, M., Chan, L.Y., et al. Substrate topography and size determine the fate of human embryonic stem cells to neuronal or glial lineage. Acta Biomater 9, 4535, 2013.

58. Ankam, S., Lim, C.K., and Yim, E.K. Actomyosin contractility plays a role in MAP2 expression during nanotopography-directed neuronal differentiation of human embryonic stem cells. Biomaterials 47, 20, 2015.

59. Yim, E.K., Pang, S.W., and Leong, K.W. Synthetic nanostructures inducing differentiation of human mesenchymal stem cells into neuronal lineage. Exp Cell Res 313, 1820, 2007.

60. Rasmussen, C.H., Reynolds, P.M., Petersen, D.R., Hansson, M., McMeeking, R.M., and Dufva, M. Enhanced differentiation of human embryonic stem cells toward definitive endoderm on ultrahigh aspect ratio nanopillars. Adv Funct Mater 26, 815, 2016.

61. Lapointe, V.L., Fernandes, A.T., Bell, N.C., Stellacci, F., and Stevens, M.M. Nanoscale topography and chemistry affect embryonic stem cell self-renewal and early differentiation. Adv Healthc Mater 2, 1644, 2013.

62. Chin, M.H., Mason, M.J., Xie, W., et al. Induced pluripotent stem cells and embryonic stem cells are distinguished by gene expression signatures. Cell Stem Cell 5, 111, 2009.

63. Hu, B.Y., Weick, J.P., Yu, J., et al. Neural differentiation of human induced pluripotent stem cells follows developmental principles but with variable potency. Proc Natl Acad Sci U S A 107, 4335, 2010.

64. Hu, S., Zhao, M.T., Jahanbani, F., et al. Effects of cellular origin on differentiation of human induced pluripotent stem cell-derived endothelial cells. JCI Insight 1, 2016.

65. Abagnale, G., Sechi, A., Steger, M., et al. Surface topography guides morphology and spatial patterning of induced pluripotent stem cell colonies. Stem Cell Reports 9, 654, 2017.

66. Harburger, D.S., and Calderwood, D.A. Integrin signalling at a glance. J Cell Sci 122, 159, 2009.

67. Hynes, R.O. Integrins: bidirectional, allosteric signaling machines. Cell 110, 673, 2002.

68. Barczyk, M., Carracedo, S., and Gullberg, D. Integrins. Cell Tissue Res 339, 269, 2010.

69. Hayashi, Y., Furue, M.K., Okamoto, T., et al. Integrins regulate mouse embryonic stem cell self-renewal. Stem Cells 25, 3005, 2007.

70. Humphrey, J.D., Dufresne, E.R., and Schwartz, M.A. Mechanotransduction and extracellular matrix homeostasis. Nat Rev Mol Cell Biol 15, 802, 2014.

71. Lv, H., Li, L., Sun, M., et al. Mechanism of regulation of stem cell differentiation by matrix stiffness. Stem Cell Res Ther 6, 103, 2015.

72. Yu, H., Lui, Y.S., Xiong, S., et al. Insights into the role of focal adhesion modulation in myogenic differentiation of human mesenchymal stem cells. Stem Cells Dev 22, 136, 2013.

73. Na, S., Collin, O., Chowdhury, F., et al. Rapid signal transduction in living cells is a unique feature of mechanotransduction. Proc Natl Acad Sci U S A 105, 6626, 2008.

74. Huveneers, S., and Danen, E.H. Adhesion signaling - crosstalk between integrins, Src and Rho. J Cell Sci 122, 1059, 2009.

75. Chowdhury, F., Na, S., Li, D., et al. Material properties of the cell dictate stress-induced spreading and differentiation in embryonic stem cells. Nat Mater 9, 82, 2010.

76. Watanabe, K., Ueno, M., Kamiya, D., et al. A ROCK inhibitor permits survival of dissociated human embryonic stem cells. Nat Biotechnol 25, 681, 2007.

77. Ohgushi, M., Matsumura, M., Eiraku, M., et al. Molecular pathway and cell state responsible for dissociationinduced apoptosis in human pluripotent stem cells. Cell Stem Cell 7, 225, 2010.

78. Ohgushi, M., Minaguchi, M., and Sasai, Y. Rho-signalingdirected YAP/TAZ activity underlies the longterm survival and expansion of human embryonic stem cells. Cell Stem Cell 17, 448, 2015.

79. Vitillo, L., Baxter, M., Iskender, B., Whiting, P., and Kimber, S.J. Integrin-associated focal adhesion kinase protects human embryonic stem cells from apoptosis, detachment, and differentiation. Stem Cell Reports 7, 167, 2016.

80. Villa-Diaz, L.G., Kim, J.K., Laperle, A., Palecek, S.P., and Krebsbach, P.H. Inhibition of focal adhesion kinase signaling by integrin alpha6beta1 supports human pluripotent stem cell self-renewal. Stem Cells 34 , $1753,2016$. 
81. Cattavarayane, S., Palovuori, R., Tanjore Ramanathan, J., and Manninen, A. alpha6beta1- and alphaVintegrins are required for long-term self-renewal of murine embryonic stem cells in the absence of LIF. BMC Cell Biol 16, 3, 2015.

82. Wade, R., Bohl, J., and Vande Pol, S. Paxillin null embryonic stem cells are impaired in cell spreading and tyrosine phosphorylation of focal adhesion kinase. Oncogene 21, 96, 2002.

83. Taleahmad, S., Mirzaei, M., Parker, L.M., et al. Proteome analysis of ground state pluripotency. Sci Rep $5,17985,2015$.

84. Ko, K.S., Arora, P.D., and McCulloch, C.A. Cadherins mediate intercellular mechanical signaling in fibroblasts by activation of stretch-sensitive calcium-permeable channels. J Biol Chem 276, 35967, 2001.

85. Martinac, B. Mechanosensitive ion channels: an evolutionary and scientific tour de force in mechanobiology. Channels (Austin) 6, 211, 2012.

86. Eisenhoffer, G.T., Loftus, P.D., Yoshigi, M., et al. Crowding induces live cell extrusion to maintain homeostatic cell numbers in epithelia. Nature 484, 546, 2012.

87. Pathak, M.M., Nourse, J.L., Tran, T., et al. Stretchactivated ion channel Piezo1 directs lineage choice in human neural stem cells. Proc Natl Acad Sci U S A 111, 16148, 2014. 88. Dupont, S., Morsut, L., Aragona, M., et al. Role of YAP/ TAZ in mechanotransduction. Nature 474, 179, 2011. 89. Yu, F.X., and Guan, K.L. The Hippo pathway: regulators and regulations. Genes Dev 27, 355, 2013. 90. Qin, H., Blaschke, K., Wei, G., et al. Transcriptional analysis of pluripotency reveals the Hippo pathway as a barrier to reprogramming. Hum Mol Genet 21, 2054, 2012.

91. Qin, H., Hejna, M., Liu, Y., et al. YAP induces human naive pluripotency. Cell Rep 14, $2301,2016$.

92. Chung, H., Lee, B.K., Uprety, N., Shen, W., Lee, J., and Kim, J. Yap1 is dispensable for self-renewal but required for proper differentiation of mouse embryonic stem (ES) cells. EMBO Rep 17, 519, 2016.

93. Sakurai, K., Talukdar, I., Patil, V.S., et al. Kinome-wide functional analysis highlights the role of cytoskeletal remodeling in somatic cell reprogramming. Cell Stem Cell 14, 523, 2014.

94. Maitre, J.L., Niwayama, R., Turlier, H., Nedelec, F., and Hiiragi, T. Pulsatile cell-autonomous contractility drives compaction in the mouse embryo. Nat Cell Biol 17, 849, 2015.

95. Samarage, C.R., White, M.D., Alvarez, Y.D., et al. Cortical tension allocates the first inner cells of the mammalian embryo. Dev Cell 34, 435, 2015.

96. Beyer, T.A., Weiss, A., Khomchuk, Y., Huang, K., Ogunjimi, A.A., Varelas, X., et al. Switch enhancers interpret TGF-beta and Hippo signaling to control cell fate in human embryonic stem cells. Cell Rep 5, $1611,2013$.

97. Lian, I., Kim, J., Okazawa, H., et al. The role of YAP transcription coactivator in regulating stem cell selfrenewal and differentiation. Genes Dev 24, 1106, 2010.

98. Wang, N., Tytell, J.D., and Ingber, D.E. Mechanotransduction at a distance: mechanically coupling the extracellular matrix with the nucleus. Nat Rev Mol Cell Biol 10, 75, 2009.

99. Nathan, A.S., Baker, B.M., Nerurkar, N.L., and Mauck, R.L. Mechano-topographic modulation of stem cell nuclear shape on nanofibrous scaffolds. Acta Biomater 7, 57, 2011.

100. Kulangara, K., Yang, J., Chellappan, M., Yang, Y., and Leong, K.W. Nanotopography alters nuclear protein expression, proliferation and differentiation of human mesenchymal stem/stromal cells. PLoS One 9, e114698, 2014.

101. Jain, N., Iyer, K.V., Kumar, A., and Shivashankar, G.V. Cell geometric constraints induce modular gene-expression patterns via redistribution of HDAC3 regulated by actomyosin contractility. Proc Natl Acad Sci U S A 110, 11349, 2013.

102. Rasmussen, C.H., Reynolds, P.M., Petersen, D.R, et al. Enhanced differentiation of human embryonic stem cells toward definitive endoderm on ultrahigh aspect ratio nanopillars. Adv Funct Mater 26, 815, 2016. 103. Eroshenko, N., Ramachandran, R., Yadavalli, V.K., and Rao, R.R. Effect of substrate stiffness on early human embryonic stem cell differentiation. J Biol Eng 7, 7, 2013.

104. Evans, N.D., Minelli, C., Gentleman, E., et al. Substrate stiffness affects early differentiation events in embryonic stem cells. Eur Cell Mater 18, 1-13; discussion 4, 2009.

105. Mei, Y., Saha, K., Bogatyrev, S.R., et al. Combinatorial development of biomaterials for clonal growth of human pluripotent stem cells. Nat Mater 9, 768, 2010.

106. Chan, L.Y., Birch, W.R., Yim, E.K., and Choo, A.B. Temporal application of topography to increase the rate of neural differentiation from human pluripotent stem cells. Biomaterials 34, 382, 2013.

107. Teo, B.K., Wong, S.T., Lim, C.K., et al. Nanotopography modulates mechanotransduction of stem cells and induces differentiation through focal adhesion kinase. ACS Nano 7, 4785, 2013. 
108. Coyer, S.R., Singh, A., Dumbauld, D.W., et al. Nanopatterning reveals an ECM area threshold for focal adhesion assembly and force transmission that is regulated by integrin activation and cytoskeleton tension. J Cell Sci 125, 5110, 2012.

109. Galli, C., Piemontese, M., Lumetti, S., Ravanetti, F., Macaluso, G.M., and Passeri, G. Actin cytoskeleton controls activation of Wnt/beta-catenin signaling in mesenchymal cells on implant surfaces with different topographies. Acta Biomater 8, 2963, 2012.

110. Niu, H., Dan, L.D., Tang, W., et al. Surface topography regulates osteogenic differentiation of MSCs via crosstalk between FAK/MAPK and ILK/b-catenin pathways in a hierarchically porous environment. ACS Biomater Sci Eng 3, 3161, 2017.

111. Chowdhury, F., Li, Y., Poh, Y.C., Yokohama-Tamaki, T., Wang, N., and Tanaka, T.S. Soft substrates promote homogeneous self-renewal of embryonic stem cells via downregulating cell-matrix tractions. PLoS One 5, e15655, 2010.

112. Dalby, M.J., Gadegaard, N., and Oreffo, R.O. Harnessing nanotopography and integrin-matrix interactions to influence stem cell fate. Nat Mater 13, 558, 2014.

113. Tzoneva, R., Faucheux, N., and Groth, T. Wettability of substrata controls cell-substrate and cell-cell adhesions. Biochim Biophys Acta 1770, 1538, 2007.

114. van Kooten, T.G., Spijker, H.T., and Busscher, H.J. Plasma-treated polystyrene surfaces: model surfaces for studying cell-biomaterial interactions. Biomaterials 25, 1735, 2004.

115. Ko, J.Y., Oh, H.J., Lee, J., and Im, G.I. Nanotopographic influence on the in vitro behavior of induced pluripotent stem cells. Tissue Eng Part A 2017. [Epub ahead of print]; DOI: 10.1089/ten.TEA.2017.0144. 116. Halder, G., Dupont, S., and Piccolo, S. Transduction of mechanical and cytoskeletal cues by YAP and TAZ. Nat Rev Mol Cell Biol 13, 591, 2012.

117. Luo, W., Shitaye, H., Friedman, M., et al. Disruption of cell-matrix interactions by heparin enhances mesenchymal progenitor adipocyte differentiation. Exp Cell Res 314, 3382, 2008.

118. Yim, E.K., Darling, E.M., Kulangara, K., Guilak, F., and Leong, K.W. Nanotopography-induced changes in focal adhesions, cytoskeletal organization, and mechanical properties of human mesenchymal stem cells. Biomaterials 31, 1299, 2010.

119. Low, B.C., Pan, C.Q., Shivashankar, G.V., Bershadsky, A., Sudol, M., and Sheetz, M. YAP/TAZ as mechanosensors and mechanotransducers in regulating organ size and tumor growth. FEBS Lett 588, 2663, 2014.

120. Hong, J.H., Hwang, E.S., McManus, M.T., et al. TAZ, a transcriptional modulator of mesenchymal stem cell differentiation. Science 309, 1074, 2005.

121. Wang, P.Y., Lian, Y.S., Chang, R., Liao, W.H., Chen, W.S., and Tsai, W.B. Modulation of PEImediated gene transfection through controlling cytoskeleton organization and nuclear morphology via nanogrooved topographies. ACS Biomater Sci Eng 3, 3283, 2017.

122. Hwang, J.H., Lee, D.H., Byun, M.R., Kim, A.R., Kim, K.M., Park, J.I., et al. Nanotopological plate stimulates osteogenic differentiation through TAZ activation. Sci Rep 7, 3632, 2017.

123. Kim, J.H., Kim, H.W., Cha, K.J., Han, J., Jang, Y.J., and Kim, D.S. Nanotopography promotes pancreatic differentiation of human embryonic stem cells and induced pluripotent stem cells. ACS Nano 10, 3342, 2016.

124. Song, L., Wang, K., Li, Y., and Yang, Y. Nanotopography promoted neuronal differentiation of human induced pluripotent stem cells. Colloids Surf B Biointerfaces 148, 49, 2016.

125. Das, R.K., and Zouani, O.F. A review of the effects of the cell environment physicochemical nanoarchitecture on stem cell commitment. Biomaterials 35, 5278, 2014.

126. Guvendiren, M., and Burdick, J.A. The control of stem cell morphology and differentiation by hydrogel surface wrinkles. Biomaterials 31, 6511, 2010.

127. Ayala, R., Zhang, C., Yang, D., et al. Engineering the cell-material interface for controlling stem cell adhesion, migration, and differentiation. Biomaterials 32, 3700, 2011.

128. Zouani, O.F., Chanseau, C., Brouillaud, B., et al. Altered nanofeature size dictates stem cell differentiation. J Cell Sci 125, 1217, 2012.

129. Bariana, M., Dwivedi, P., Ranjitkar, S., Kaidonis, J.A., Losic, D., and Anderson, P.J. Biological response of human suture mesenchymal cells to Titania nanotube-based implants for advanced craniosynostosis therapy. Colloids Surf B Biointerfaces 150, 59, 2017.

130. Liu, L., Bhatia, R., and Webster, T.J. Atomic layer deposition of nano-TiO2 thin films with enhanced biocompatibility and antimicrobial activity for orthopedic implants. Int J Nanomedicine 12, 8711, 2017. 
131. Carson, D., Hnilova, M., Yang, X., et al. Nanotopographyinduced structural anisotropy and sarcomere development in human cardiomyocytes derived from induced pluripotent stem cells. ACS Appl Mater Interfaces 8, 21923, 2016.

132. Kim, D.H., Kshitiz, Smith, R.R., Kim, P., et al. Nanopatterned cardiac cell patches promote stem cell niche formation and myocardial regeneration. Integr Biol (Camb) 4, 1019, 2013.

133. Shiba, Y., Gomibuchi, T., Seto, T., et al. Allogeneic transplantation of iPS cell-derived cardiomyocytes regenerates primate hearts. Nature 538, 388, 2016.

134. Yang, H.S., Ieronimakis, N., Tsui, J.H., et al. Nanopatterned muscle cell patches for enhanced myogenesis and dystrophin expression in a mouse model of muscular dystrophy. Biomaterials 35, 1478, 2012.

135. Mashinchian, O., Bonakdar, S., Taghinejad, H., et al. Cellimprinted substrates act as an artificial niche for skin regeneration. ACS Appl Mater Interfaces 6, 13280, 2014.

136. Cranford, S.W., de Boer, J., van Blitterswijk, C., and Buehler, M.J. Materiomics: an -omics approach to biomaterials research. Adv Mater 25, 802, 2013.

137. Yang, C., DelRio, F.W., Ma, H., et al. Spatially patterned matrix elasticity directs stem cell fate. Proc Natl Acad Sci USA 113, E4439, 2016.

138. Pathak, A., and Kumar, S. Independent regulation of tumor cell migration by matrix stiffness and confinement. Proc Natl Acad Sci U S A 109, 10334, 2012.

139. Unadkat, H.V., Hulsman, M., Cornelissen, K., et al. An algorithm-based topographical biomaterials library to instruct cell fate. Proc Natl Acad Sci U S A 108, 16565, 2011.

140. Anderson, D.G., Levenberg, S., and Langer, R. Nanoliterscale synthesis of arrayed biomaterials and application to human embryonic stem cells. Nat Biotechnol 22, 863, 2004.

141. Khan, F., Tare, R.S., Kanczler, J.M., Oreffo, R.O., and Bradley, M. Strategies for cell manipulation and skeletal tissue engineering using high-throughput polymer blend formulation and microarray techniques. Biomaterials 31, 2216, 2010.

142. Chickarmane, V., and Peterson, C. A computational model for understanding stem cell, trophectoderm and endoderm lineage determination. PLoS One 3, e3478, 2008.

143. Woolf, P.J., Prudhomme, W., Daheron, L., Daley, G.Q., and Lauffenburger, D.A. Bayesian analysis of signaling networks governing embryonic stem cell fate decisions. Bioinformatics 21, 741, 2005.

144. Chavez, L., Bais, A.S., Vingron, M., Lehrach, H., Adjaye, J., and Herwig, R. In silico identification of a core regulatory network of OCT4 in human embryonic stem cells using an integrated approach. BMC Genomics 10, 314, 2009.

145. Ellison, D., Munden, A., and Levchenko, A. Computational model and microfluidic platform for the investigation of paracrine and autocrine signaling in mouse embryonic stem cells. Mol Biosyst 5, 1004, 2009.

146. Consolo, F., Bariani, C., Mantalaris, A., Montevecchi, F., Redaelli, A., and Morbiducci, U. Computational modeling for the optimization of a cardiogenic 3D bioprocess of encapsulated embryonic stem cells. Biomech Model Mechanobiol 11, 261, 2012.

147. Scholma, J., Schivo, S., Urquidi Camacho, R.A., van de Pol, J., Karperien, M., and Post, J.N. Biological networks 101: computational modeling for molecular biologists. Gene 533, 379, 2014.

148. Xiong, F., and Megason, S.G. Abstracting the principles of development using imaging and modeling. Integr Biol (Camb) 7, 633, 2015.

149. Brodland, G.W. How computational models can help unlock biological systems. Semin Cell Dev Biol $47,62,2015$ 


\section{Chapter III}

\section{Screening as a Strategy to drive Regenerative Medicine Research}

Steven Vermeulen ${ }^{1,2}$ and Jan de Boer ${ }^{2}$

${ }^{1}$ Laboratory for Cell Biology-Inspired Tissue Engineering, MERLN Institute, University of Maastricht, Maastricht, The Netherlands.

${ }^{2}$ BioInterface Science Group, Department of Biomedical Engineering and Institute for Complex Molecular Systems, University of Eindhoven, Eindhoven, The Netherlands

Published in: Methods (2020) 


\section{Abstract}

In the field of regenerative medicine, optimization of the parameters leading to a desirable outcome remains a huge challenge. Examples include protocols for the guided differentiation of pluripotent cells towards specialized and functional cell types, phenotypic maintenance of primary cells in cell culture, or engineering of materials for improved tissue interaction with medical implants. This challenge originates from the enormous design space for biomaterials, chemical and biochemical compounds, and incomplete knowledge of the guiding biological principles. To tackle this challenge, high-throughput platforms allow screening of multiple perturbations in one experimental setup. In this review, we provide an overview of screening platforms that are used in regenerative medicine. We discuss their fabrication techniques, and in silico tools to analyze the extensive data sets typically generated by these platforms. 


\section{Introduction}

A challenge in regenerative medicine is to control the complexity of biological systems. Advances have been made to control cell behavior leading to clinical applications e.g., the differentiation of pluripotent cells towards primary cells ${ }^{1}$ or the use of bioactive graft materials ${ }^{2}$. Many opportunities and challenges remain, such as the high incidence of catheter implant infections ${ }^{3}$, the foreign body response against biomaterials ${ }^{4}$, and the standing challenge to engineer functional tissues and organs ex vivo. Progress in these and other fields of regenerative medicine can be expedited if we can overcome current limitations in material design and increase our knowledge of biomaterial-cell interactions. Materials need to meet some requirements, for example, durability is a crucial factor in determining the success of the implant when supporting or replacing tissues ${ }^{5}$. At the same time, materials should not be cytotoxic or cause long-term harm in the body, as is often seen with breast implant ruptures or pathological encapsulation ${ }^{6}$. Furthermore, cell-biomaterial interaction should favor matrix deposition that allows optimal implant integration. Both physical and chemical properties can affect cell behavior. For example, a promising approach for bone tissue regeneration is the use of calcium phosphate particles, of which both chemistry (calcium phosphate) and structural properties (surface roughness) activate transcriptional profiles associated with bone induction ${ }^{7}$. This also reflects a challenge in finding the most optimal material design due to a large number of design parameters. For example, hundreds of different chemical compositions exist of the biocompatible and clinically applied material polyurethane ${ }^{8}$.

To address the issues associated with incomplete information on both the biological and material side of the interface, methodologies that vary a large number of perturbations in one experimental setup can rapidly increase knowledge. Below, we highlight the potential of screening approaches where biology, material engineering, and computational science converge.

\section{Biomaterials for High-Throughput Applications}

During development and in the adult body, cells receive a wide variety of signals from their external environment. Therefore, much research focusses on appropriately controlling cell signaling in vitro for guiding cell behavior. This is not trivial because cells in standard culture conditions are exposed to different stimuli compared to physiological conditions. For example, standard culture media contains animal-derived serum, thus exposing cells to a-physiological cytokines and growth factors. In addition, flat tissue culture polystyrene has unique chemical and physical properties, which influence cell behavior. Therefore, it is not surprising that transferring cells from their native environment into cell culture often leads to substantial loss of in situ phenotypical identity. For example, chondrocytes that maintain cartilage homeostasis, quickly lose morphological characteristics and chondrogenic gene expression when cultured on tissue culture plastic ${ }^{9}$. Although an undesirable result, this does indicate that the chemical and physical properties of the substrate on which cells grow has a profound effect on cell physiology and shows that we can use these properties to tweak cell behavior. In this section, we discuss different 
cell-biomaterial interfaces in a high-throughput setting in which large numbers of possibilities in chemical, biochemical, and structural biomaterial designs are used to control cells.

\section{Surface Structures}

Standard cell culture is carried out on flat surfaces. For cells, this is fundamentally different from their native 3D tissue environment. Therefore, physical cues in the form of surface structures are a promising tool for controlling cell behavior. However, a challenge is identifying the most optimal surface design that elicits a particular response. Surface structures can be constructed in variable height profiles, in a disordered or ordered manner, with different geometries, or by combining multiple profiles, thus creating hierarchical structures. Screening approaches can provide essential insight into this highly complex design space. In Figure 1, we provide the reader with a visualization of some high-throughput platforms, which we will be discussing in this section.

A

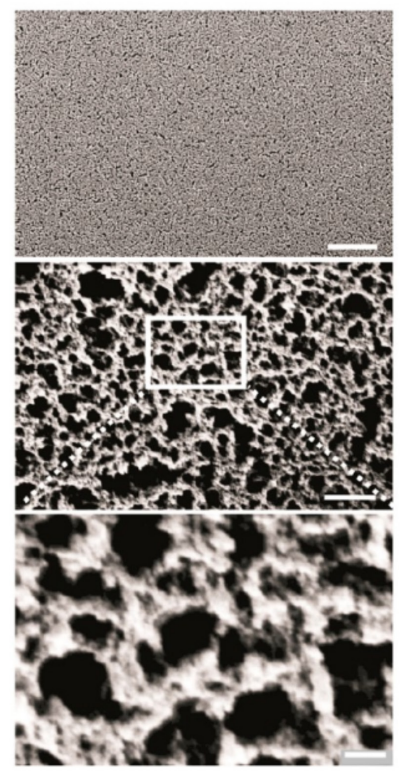

B
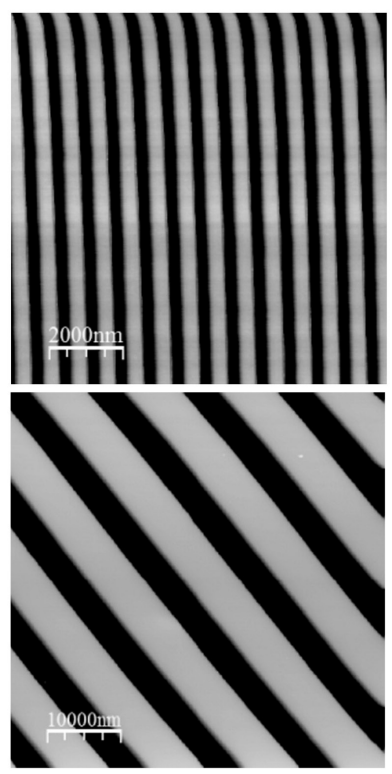

C
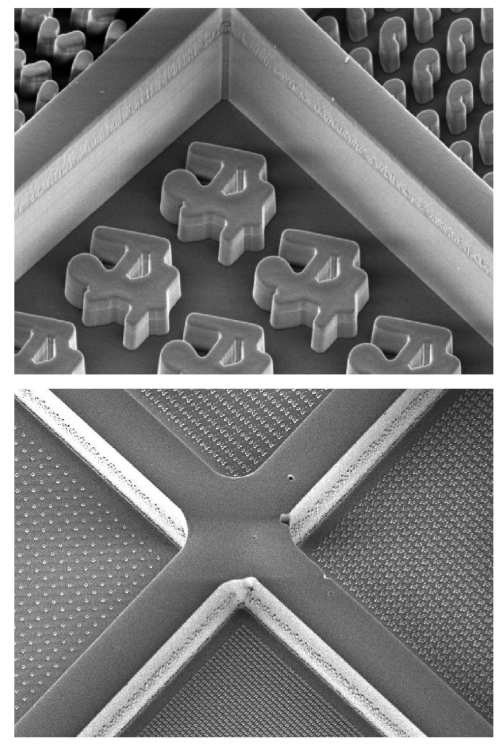

$10 \mu \mathrm{m}$

Figure 1: Examples of different surface architectures. A) SEM images of a surface containing roughness levels of $68+-30 \mathrm{~nm}$. Scale bars: top image, $5 \mu \mathrm{m}$; middle image, $500 \mathrm{~nm}$; bottom image, 100 $\mathrm{nm}$. These hierarchical micro- and nanoroughness levels can promote ESC pluripotency. Adapted with permission from ${ }^{15}$. Copyright (2015) American Chemical Society. B) AFM image of groove structures with a groove width of $350 \mathrm{~nm}$ (up) and $5 \mu \mathrm{m}$ (down) known to modulate neuronal differentiation. Adapted with permission from ${ }^{211}$. Copyright (2013) Elsevier. C) Upper image represents a SEM image of the TopoChip platform, a high-throughput platform containing 2176 unique micro-structural designs of $10 \mu \mathrm{m}$ height and separated by walls of $30 \mu \mathrm{m}$. The bottom image represents the nanoTopoChip, a highthroughput platform containing 1246 unique nano-structural designs separated by walls of $30 \mu \mathrm{m}$. Adapted with permission from ${ }^{52}$. Copyright (2017) Elsevier. 


\section{Roughness}

Surface roughness can be regarded as topographies with randomly distributed height patterns in micro- or nanometer dimensions. This random feature distinguishes itself compared to other surface types, where the topographical dimensions are precisely controlled. Material roughness is mostly associated with bone tissue engineering and can be introduced to the surface by sandblasting and acid-etching, resulting in superior osteo-integrative capabilities compared to smooth surface titanium implants ${ }^{10,11}$. It is, therefore, not surprising that osteoblast-like cells and MSCs demonstrate improved osteogenic differentiation potential on rough surfaces ${ }^{12,13}$. Also, calcium phosphate surfaces that closely mimic the bone microenvironment, both at a chemical and topographical level, improve the osteogenic potential of MSCs ${ }^{14}$ and the osteogenic cell line MG63 ${ }^{7}$. Roughness is utilized for studying other types of cell-material interactions as well. Here, it is interesting to mention that for embryonic stem cells, materials with combined micro- and nanometer roughness ${ }^{15}$ or nanometer roughness alone ${ }^{16}$ promotes pluripotency. Also, proliferation capabilities can improve on nanorough materials, as shown for endothelial cells ${ }^{17}$.

For surface roughness, screening is limited to gradient formats due to their simple design. Through the use of gradients, increased roughness coincides with enhanced proliferation for osteoblasts, while showing the opposite effects for fibroblasts ${ }^{18}$. For osteogenic differentiation, an optimal roughness niche was identified for MSCs ${ }^{13}$.

\section{Curvature}

Curvature can exist as concave (inward curve) and convex shapes (outward curve). Curvature can influence MSC migration and differentiation 19 and is a useful tool for studying mechanobiology 20,21 , since similarities can be found between curvatures in vitro and in vivo ${ }^{22}$. The effect of curvature on cell behavior is studied in a screening format through the use of wrinkles, for instance, by Zhou and colleagues, who used wrinkles in nanometer dimensions to optimize parameters for osteoblasts attachment ${ }^{23}$. This concept can be expanded in micrometer dimensions and in a 96-well plate format allowing the possibility for combining curvature with different culture media combinations ${ }^{24}$. Furthermore, combinations with other perturbations is achievable through coating wrinkles with, for example, inorganic biocompounds 25 .

\section{Grooves}

Grooves are continuous lines with variable dimensions across the material surface, which are mainly associated with contact-guidance of cells, i.e., they influence cell orientation. Besides orientation, other cell morphological characteristics such as cell area are affected depending on the proximity and depth of these grooves ${ }^{26}$. In general, grooves can be build in micro- and nano dimensions with varying height profiles, of which both the grooves and ridge length are variable, and even in hierarchical format combining both dimensions ${ }^{27}$. Grooves can also be placed in perpendicular orientations, which allows the construction of gratings that, depending on the dimensions, gives rise to more complex topographical surface structures. 
Microgrooves influence cell behavior in very distinct ways. For example, an increase in global histone acetylation resulted in improving the reprogramming process of fibroblasts towards iPSCs ${ }^{28}$. Grooves influence the differentiation potential of pluripotent cells, with nanogrooves guiding BMP4-induced differentiation of iPSCs ${ }^{29}$, the differentiation of ESCs into neurons ${ }^{30}$, and the differentiation of astrocytes into glia-like cells ${ }^{31}$. Furthermore, phenotypical characteristics of primary cells can improve on grooves. This is demonstrated by the increased expression of tendon-related genes in tenocytes when cultured on nanogrooves ${ }^{32}$. A similar observation was found with microgrooves, which besides cellular alignment, also aligned collagen-I, the main protein component of the tendon ${ }^{33}$. A similar concept was applied for neuronal cells, where axonal outgrowth orientates according to the grooves direction ${ }^{34}$. Both examples can mimicking the in vivo tissue organization, a useful property for tissue-engineering applications.

Because the dimensionality of grooves is strongly variable, screening approaches have been implemented to determine which dimensions are best suited for obtaining a particular cell phenotype. This is illustrated by a screening platform containing 25 units with grooves and ridges in different micrometer dimensions, termed the polyimide (PI) chip. The PI chip was used to identify groove structures that stimulate adipogenic or osteogenic differentiation ${ }^{26}$. The integrated mechanobiology platform (IMP) utilizes gratings and grids in a 96-well plate format, thus enabling the use of molecular biology analysis tools such as qPCR or ELISA. The pitch size of the gratings varies between 500 and $3000 \mathrm{~nm}$, while on the grid geometries the trench width can be varied between $100-300 \mathrm{~nm}$. This platform was used to demonstrate that surface structures can influence IL-2 secretion in T cells, an important cytokine ${ }^{35}$. A screening platform containing both variable micro- and nano grooves is the multi-architectural chip (MARC). It is noteworthy to mention that this platform contains only 18 surface structures, yet besides grooves also contains pillars and pits, thereby creating a broad surface diversity. Surfaces from the MARC were identified that promoted differentiation of neural progenitor cells ${ }^{36}$ and ESCs ${ }^{37}$ towards either adult neurons or glial cells. Differentiation towards dopaminergic neurons 38 was associated with anisotropic patterns (gratings), while isotropic patterns (pillars and wells) supported glial differentiation.

\section{Complex topographies}

Topographies can be designed and produced in increasing complexity at both the micro- and nanometer scale. With complexity also comes larger numbers of potential designs. These designer topographies can, as roughness, curvature, and grooves do, profoundly influence cell behavior, as illustrated by the influence of disordered nano-topographies on MSC differentiation towards the osteogenic lineage ${ }^{39}$. The height of topographies influences cell phenotype, as shown for nano-topographies with high aspect ratios that support ESC differentiation towards the endoderm lineage in contrast to smaller topographies ${ }^{40}$. Besides enhancing differentiation, pluripotency can also be supported by nano-topographies in honeycomb and hexagonal configurations ${ }^{41}$. These examples demonstrate that a large variation exists in the topographical 
design space, emphasizing the need for high-throughput approaches to find the most optimal surface structure for a given application.

As a first example, the micro-topographical BioSurface Structure Array (BSSA) contains 504 unique topographical designs with variable spacing between circles or squares, and with variable heights of $0.6,1.6$, and $2.4 \mu \mathrm{m}$. This platform has been used to explore the effect on osteogenic differentiation with positive results obtained for an osteoblastic cell line ${ }^{42}$ and dental pulp MSCs 43. Also, topographies were identified that promoted the pluripotency of ESCs ${ }^{44}$. Besides these studies, the BSSA platform found that topographies influence the proliferation of chondrocytes and fibroblasts 45,46 .

A high-throughput platform designed by our group, known as the TopoChip, is a $2 \times 2 \mathrm{~cm}^{2}$ platform containing 2176 unique micro-topographies and 4 flat control surfaces ${ }^{47}$. The in silico design of these topographies uses three primitives: circles, triangles, and squares. Combining these primitives while varying the number, size, and orientation leads to millions of potential designs. The platform has been used extensively for studying the behavior of cells on microtopographies while enabling the identification of design parameters associated with a desired phenotype. We identified micro-topographies that promote enhanced clonogenicity of pluripotent iPSCs ${ }^{48}$, augment differentiation of tonsil-derived MSCs towards fibroblastic reticular cells ${ }^{49}$, improving the osteogenic potential of MSCs ${ }^{50}$, or improve phenotypic maintenance of tenocytes ${ }^{51}$. In addition to the TopoChip, a nano-topography version was

A

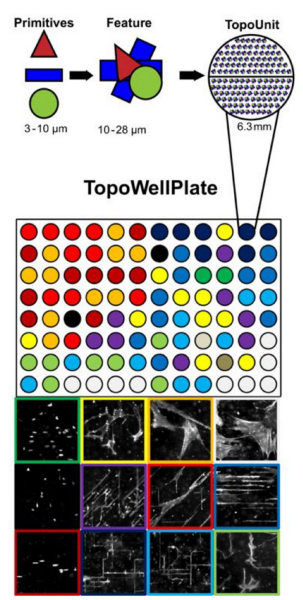

B
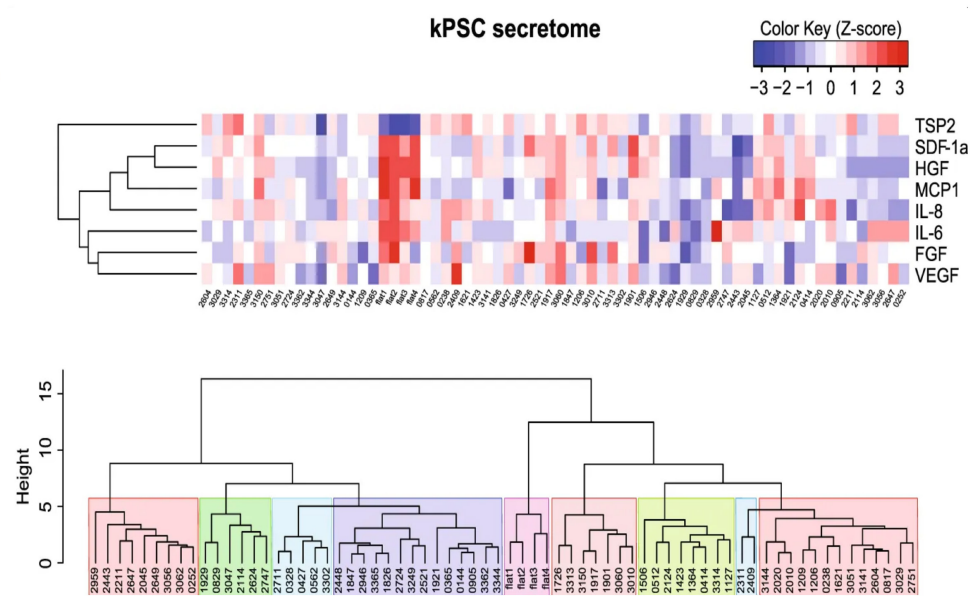

Figure 2: Multiplex ELISA applied in combination with the TopoWell Plate, a high-throughput platform. Applying a multiplex ELISA targeting 8 cytokines and growth factors on this platform allowed inferring if these micro-topographies influence the cell secretome. A) The TopoWell Plate contains 87 unique surface topographies and 9 flat surfaces as a control. Seeding kidney derived perivascular stromal cells on these surfaces induces a large variety in morphological characteristics. B) Heat map visualization representing the influence of each topography on the expression of these cytokines and growth factors. Through clustering algorithms, micro-topographical surfaces can be grouped based on their secretome profile. Adapted with permission from ${ }^{54}$. Copyright (2018) Nature Publishing Group. 
created with 1246 unique designs ${ }^{52}$. Furthermore, a 96-well platform was developed with each well containing a unique micro-topographical design, enabling other experimental techniques such as ELISA, qPCR or western blot ${ }^{53}$. Combining this so-called TopoWell platform with multiplex ELISA allowed us to associate the secretion profile of mesenchymal and perivascular stromal cells with topographical design and cell shape ${ }^{54}$ (Figure 2).

\section{Surface Biochemistry}

Cells interact with their surrounding tissue matrix in vivo. Some of these interactions are mediated by cell adhesion molecules called integrins, which recognize and bind peptide motifs present in proteins of the surrounding matrix. The RGD sequence is such a well-known peptide motif and is found on multiple matrix proteins, including fibronectin and fibrinogen ${ }^{55}$. Integrins are also capable of specifically recognizing the matrix proteins collagen-I ${ }^{56}$ and laminin ${ }^{57}$. Matrix proteins can be presented to cells as a coating and lead to different modes of attachment and signaling. For example, a combination of laminin and poly-L-lysine coating improves the differentiation of PC12 cells towards neuron-like cells ${ }^{58}$. Also, a collagen-I or poly-D-Lysine coating supports pluripotency of ESCs, while laminin or fibronectin activates integrins and downstream pathways associated with differentiation ${ }^{59}$.

Besides uniform surface coating, it is possible to create patterned areas with the matrix protein of interest. One strategy is to produce a hydrophobic, cell-repellent surface with islands of matrix proteins, with cells adhering only to protein-containing areas and adjusting their morphology based on the pattern design. The effects of matrix compositions and morphology on cell phenotype can thus be investigated. One of the earliest attempts to utilize this system is from the mid-'90s and involved laminin islands with different surface areas. The authors demonstrated that pattern design influences cell spreading and proliferation ${ }^{60}$. Later, it was shown that the differentiation potential of MSCs is altered when cultured on adhesive islands. Cells grown on smaller patterns had a stronger tendency for adipogenic differentiation, while a larger surface area favors osteogenesis ${ }^{61}$. Besides MSCs, the differentiation potential of other stem cell types, such as epidermal stem cells, can be influenced by utilizing adhesive islands ${ }^{62}$.

Due to the large number of matrix components, their possible combinations, and the large pattern design options, high-throughput methods offer an ideal opportunity to find the most optimal biochemical environment. An interesting example is a study where the effects of 32 different combinations of collagen-I, collagen-III, collagen-IV, laminin, and fibronectin on hepatocyte phenotype and differentiation of ESCs towards hepatocyte progenitor cells was determined. The approach found that certain mixtures of ECM compounds had positive synergistic effects on hepatocyte phenotype and ESC differentiation ${ }^{63}$. In Figure 3 A-C, we provide the reader with a visual representation of this ECM compound array. In another study, an array of 18 peptide-terminated SAM array elements, each with a unique laminin peptide sequence, allowed determining which sequence is responsible for promoting ESC pluripotency 64. Besides utilizing matrix proteins, extra complexity can be added by including signaling proteins such as growth factors. This was accomplished on an array platform with 44 different 
combinations of signaling proteins, on which ESC neurogenic differentiation was investigated 65. In a similar study, an array of 25 different substrates was used to find that the effect of growth factors was modulated depending on the underlying matrix compositions ${ }^{66}$.

The examples above illustrate the usefulness of screening matrix compounds, especially in combination with soluble cues. Concerning the dimensions of these adhesive islands, we reason that varying this in a high-throughput matter can be of substantial biological importance, especially when including different matrix compounds are included. Research in this niche can easily be accomplished by utilizing the same lithographical masks for the fabrication of other high-throughput biomaterial technologies. We recently developed such a high-throughput design with RGD peptides based on the TopoChip design (Figure 3 D-E).

A



D

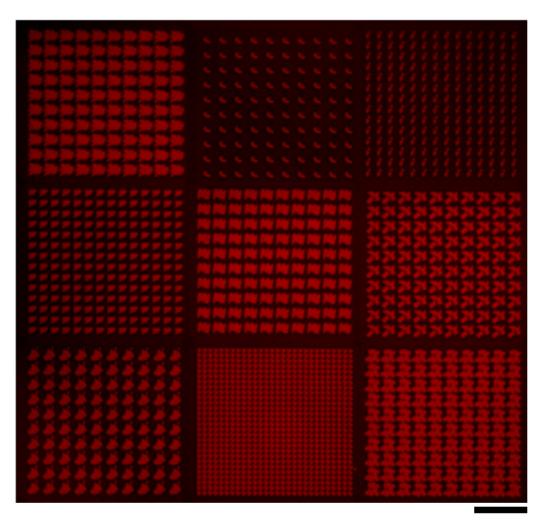

B

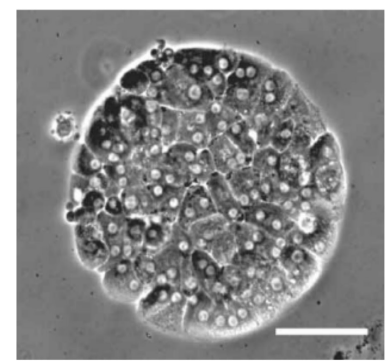

E
C

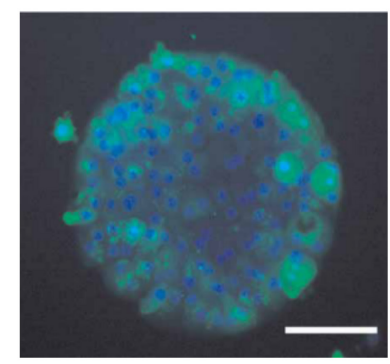



Figure 3: High-throughput screens involving ECM compounds. A) Life/Death assay on hepatocytes cultured on an array of 32 different ECM compositions. Scale bar represents $1 \mathrm{~mm}$. B) High-magnification phase-contrast and C) Fluorescent image of a single island. Green represents calcein AM and blue the nucleus through a DAPI counterstain. Scale bars represent $50 \mu \mathrm{m}$. Adapted with permission from ${ }^{63}$. Copyright (2005) Nature Publishing Group. D) RGD islands in a high throughput platform in geometric shapes of varying parameters. The design is based on a mask for creating the TopoChip platform. Scale bar represents $100 \mu \mathrm{m}$. E) CellPaint assay on BM-hMSCs grown on this substrate allows studying the influence of different densities of RGD islands on cell morphology in a high-throughput and high-content format (picture courtesy of Urandelger Tuvshindorj from the MERLN Institute, Maastricht University). 


\section{Surface Chemistry}

Surface chemistry has a strong effect on cell behavior and can be used to endow medical implants with specific bio-active properties. However, the relationship between surface chemistry and cell response is difficult to model. Therefore, high-throughput strategies to study the effect of surface chemistry on protein adsorption or cell function are of great interest. In cell culture, surface chemistry has a strong effect on cell behavior. Standard cell culture is carried out on tissueculture plastic, which is an oxygen plasma-treated polystyrene material. Oxygen treatment changes the chemistry of the material by incorporating oxygen radicals, which hydrophilizes the surface, thus improving cell-substrate attachment ${ }^{67}$, cell-cell contacts ${ }^{68}$, and proliferation ${ }^{69}$. Through lithography, this concept can be implemented for creating shapes that facilitate cell attachment ${ }^{70,71}$. Also, gradients can be produced to find the most optimal oxygen incorporation and roughness level for a particular phenotype ${ }^{72}$. The link between surface chemistry and the response it elicits on cells may be through differential deposition of serum matrix proteins such as fibronectin that adsorb on the substrate ${ }^{73}$. Surface chemistry can change the conformational structure of fibronectin, which in turn affects the binding affinity to integrins and thereby influences cell adhesion ${ }^{74}$. Nevertheless, surface chemistry might affect cell behavior directly through new and unknown mechanisms or mimic the effect of biological molecules, similar as seen with small-molecule screens.

A large variety of monomers exists, the building blocks of polymers. These monomers can be combined through chemical reactions into various polymers with different chemical and physical properties. The amount of possible polymer variation is enormous, which is illustrated in a highthroughput study where 576 novel polymers were created by different combinations of 25 monomers, each carrying the same chemical acrylate backbone ${ }^{75}$. Besides acrylates, other polymers exist that are popular for biomedicine applications such as polyesters and polyurethanes (PU), which are biocompatible and biodegradable. For PU, a polyurethane library was created with 278 different glycol backbones for studying their effect on wettability ${ }^{76}$. A similar approach was utilized with 44 different poly-acrylates for determining their affinity with fibrinogen ${ }^{77}$. An overview regarding the different polymers used for biomedical applications and their advantages and disadvantages is excellently reviewed elsewhere ${ }^{78}$.

The previously presented examples did not involve cell-based experiments. Cell behavior can give a more accurate representation of the in vivo properties of the surface chemistry on tissue engineering constructs and medical devices by measuring properties such as toxicity, adhesion, and matrix deposition. For example, by utilizing 60 different polymers based on different combinations of polyurethane, polyols, diisocyanates, and variable chain extenders, their compatibility with epithelial cell adhesion was determined ${ }^{79}$. Also, in an extensive highthroughput manner involving 1152 different polymers, the adhesion properties of these polymers against MSCs, neuronal stem cells, and chondrocytes were studied ${ }^{80}$. In this study, only 24 polymers were blended, including PGLA and PLA, which are frequently used in regenerative medicine. Cell adhesion of suspension cells can also be accomplished through polymer coatings, as demonstrated in a study utilizing a library of 210 different polyurethanes 
and 58 polyacrylates. Here, the authors identified polymers that promoted adhesion and proliferation ${ }^{81}$.

Polymer composition can be also be used to control specific cell behavior in vitro, e.g., to improve proliferation or stem cell differentiation ${ }^{82}$. We mentioned before the construction of a library of 576 polymers based on 25 different acrylate monomers ${ }^{75}$. The same library contained chemical compositions promoting endothelial differentiation of ESCs ${ }^{83}$. Utilizing different blends of PDLA and PCL, both FDA approved biomaterials, it became possible to influence ALP levels of two osteogenic cell lines ${ }^{84}$. In a library of 120 varieties of polyurethane, polymers were identified that enabled the preferential binding of an MSC subpopulation with increased osteogenic potential ${ }^{8}$. Through a library of 141 homopolymers and 400 co-polymers, the effect of these substrates on dental pulp stem cell differentiation was assessed ${ }^{85}$. Of interest, the authors managed to predict the behavior of these cells on the polymers tested through computation models. Polymers also allow the phenotypic maintenance of primary cells, as demonstrated by a screen utilizing a library of 380 different polyurethanes and acrylates ${ }^{86}$.

Chemistry can influence the phenotypical maintenance of ESCs, as demonstrated in a study applying 496 polymer blends based on 22 different acrylate combinations ${ }^{87}$. Through in-depth characterization of polymer properties, such as wettability, roughness, and elasticity, associations were made with cell phenotype. In Figure 4, we provide the reader with high-throughput data visualization from this study. Furthermore, they found that vitronectin from the culture media adsorbed to the polymers, and resulted in a specific integrin-binding, which initiated the cell signaling that promoted pluripotency. In parallel with the study, this research group studied embryoid body adhesion on the same polymer array. Of interest is that here both the preadsorbed fibronectin and the bare polymer were responsible for controlling cell adhesion ${ }^{88}$.

Another clinically relevant application are polymers with properties that prevent implant infection. Based on the previous library for studying the behavior of ESCs and EB's, structurally related polymers were identified that resist bacterial attachment, which was further validated by in vivo testing ${ }^{89}$. Follow-up studies demonstrated a clear relationship between the chemical composition of the polymers and antimicrobial properties ${ }^{90}$. Also interesting to mention is that the same group applied a "multi-generation" screening, whereby first homopolymers from a library of 116 acrylate monomers were identified that resist antibacterial attachment ${ }^{91}$. Afterwards, these hit monomers were mixed in polymer format to conduct a second screen, after which hit polymers were selected to make composite materials.

Until now, we focused on chemical characteristics that influence protein adsorption, cell behavior, and microbial attachment. However, in the context of medical implants, degradation, durability, and physiomechanical properties are relevant parameters for clinical performance as well. Assessing these characteristics in a high-throughput context is a challenging endeavor, which Kohn and co-workers achieved by simultaneously assessing the polymer structure of 144 polymers, their glass transition temperature, hydrophobicity, mechanical properties, and fibroblast proliferation ${ }^{92}$. 

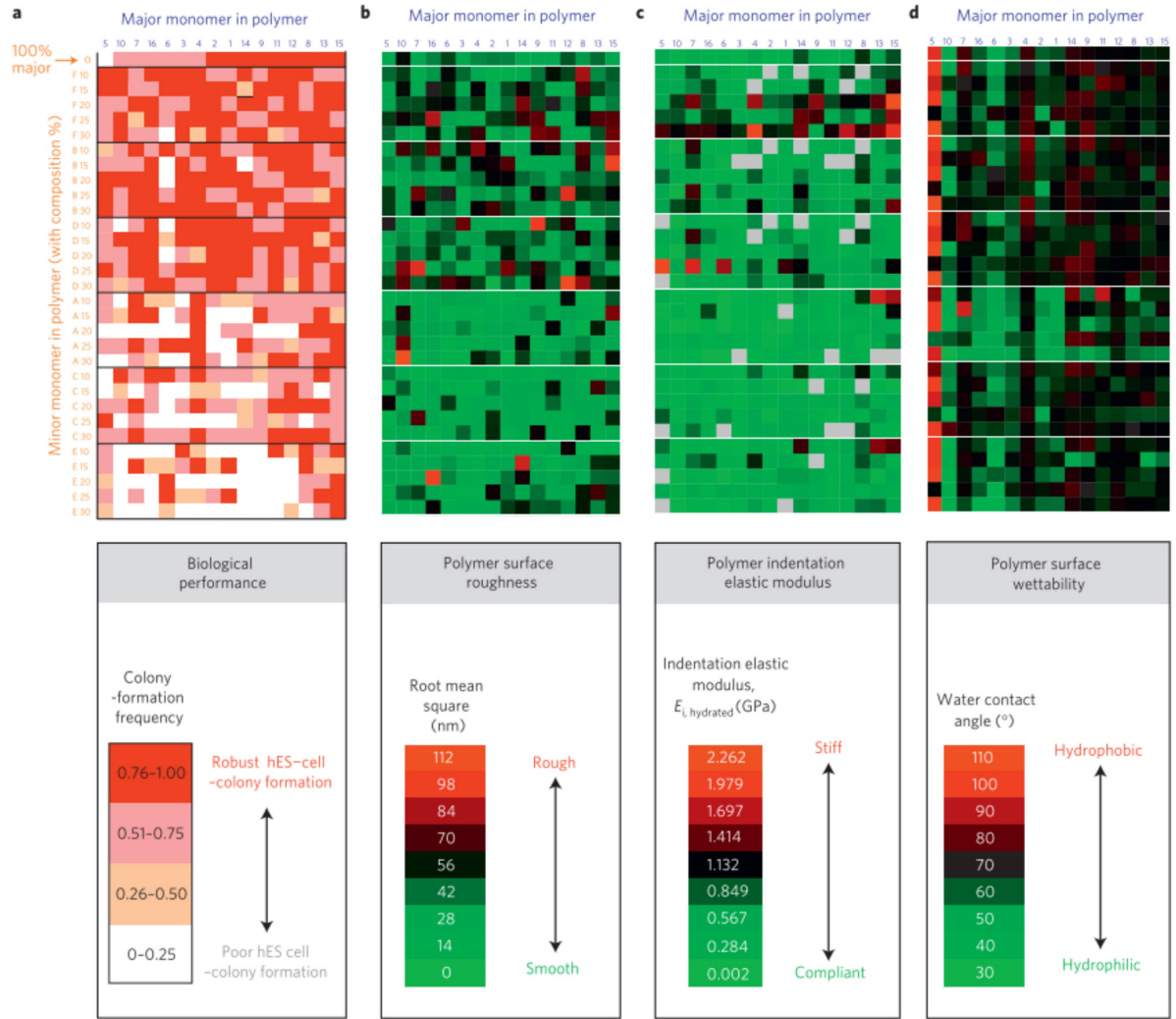

Figure 4: Example of a visualization of a high-throughput platform mapping the biological response of ESCs against 496 different polymers. Through measuring surface roughness, elasticity, and wettability, functional relationships between surface characteristics and cell phenotype can be measured. Here, a positive relationship between ESC colony formation and moderate wettability was established. Adapted with permission from ${ }^{87}$. Copyright (2010) Nature Publishing Group.

\section{Hydrogels}

Hydrogels are a popular tool for guiding cell behavior through the possibility to simultaneously control physical, chemical, and biochemical parameters. The main difference between the previous paragraphs is that hydrogels can provide a $3 \mathrm{D}$ environment, thereby more closely resembling the in vivo context of some tissue types. In hydrogels, molecules for cell signaling can be attached to a flexible backbone with dynamic physical properties. This allows regulating the stiffness of the gel, which is a crucial regulator of cell behavior, as demonstrated by the landmark paper of Disher and co-workers ${ }^{93}$. In this study, hydrogels that match tissue elasticity drives MSC differentiation towards the matching cell types. Also, for pluripotency of stem cells, the stiffness of the surrounding environment can be a crucial factor ${ }^{94}$.

Since parameters such as biodegradability, biomolecule presentation, growth factor release, and mechanical properties can be varied, high-throughput approaches help determine the most 
optimal parameters for controlling cell fate in hydrogels. This is demonstrated by a microwell platform with modular stiffness, where individual microwells can be functionalized with a different protein combination ${ }^{95}$. Environmental niches promoting the differentiation of MSCs and the self-renewal of neuronal stem cells were discovered with this platform. This concept was further expanded by fluctuating stiffness levels, gel degradability, cell density, ECM compounds, soluble factors, and cell-cell interaction components, thereby creating more than 1000 different niche environments that were assessed on their potential for controlling ESC pluripotency ${ }^{96}$. Also, combinational arrays can be generated where polymer concentration, peptide presentations, and growth factor concentrations are varied. This allowed the identification of an optimal niche for myofibrogenesis in human mesenchymal stromal cells ${ }^{97}$. The same group further demonstrated that also mechanical stimulation can be applied on hydrogels in an array format, whereby implemented sensors allowed monitoring the progress of hydrogel stiffness changes in real-time ${ }^{98}$.

Other material fabrication parameters that can be controlled in hydrogel synthesis are monomer blending, $\mathrm{pH}$, and crosslinker concentration. In an array system, 80 different polymer blends where combined resulting in gels with different stiffness and elastic properties ${ }^{99}$. Thermoresponsive properties are interesting for clinical applications because they can be used to release cells from the hydrogel, for example, after injection. A high-throughput screen for identifying thermoresponsive hydrogels was performed by Zhang and colleagues. The authors tested 2280 different polymer compositions and identified hydrogels with optimal properties for cell attachment and temperature controlled release ${ }^{100}$.

An exciting opportunity for clinical application is the use of hydrogels in microbead format. Due to the small size of these beads, nutrient and gas exchange is facilitated, while cells are in direct contact with a physiologically relevant matrix. In this context, the optimal elasticity of agarosebased microbeads was identified using a high-throughput approach ${ }^{101}$. Similarly, a highthroughput screen identified optimal parameters for an MSC and hepatocyte co-culture inside alginate-based microbeads ${ }^{102}$. This study further found improved liver enzyme levels after implanting these co-culture microbeads in rats, suggesting that the approach does have potential clinical applications in the future for restoring tissue and organ function.

\section{Micro-Fluidics and Droplet Technologies}

Microfluidics is defined as the control of fluids in micrometer dimensions. By working in these dimensions, microfluidics enables the control of experimental parameters in the cell size range. Due to these small dimensions, microfluidics come hand in hand with lab-on-a-chip technology and hold the promise of influencing a large number of parameters on a small platform. The architecture of the microfluidic platform is modifiable and allows, for instance, dynamic control of growth factor or cytokine gradients ${ }^{103}$. Furthermore, it is possible to apply different surface (bio)chemistries ${ }^{104}$ and surface structures 105 inside the platform. Some platforms have included stretching as a biological signal mimicking in vivo conditions ${ }^{106}$. An extra layer of complexity can be introduced through compartmentalization, in which different separate cell systems are 
connected, which moves the platform more towards an organ-on-a-chip concept. These examples demonstrate that the microfluidic lab-on-a-chip concept is a highly versatile and attractive platform for high-throughput research in a regenerative medicine context ${ }^{107}$.

Micro-fluidics research in a high-throughput setting often involves the use of compound gradients. For instance, drug screening was performed on cancer cells to asses their effect on migration kinetics ${ }^{108}$. In a similar approach, Zhang and colleagues utilized 3120 microchambers to monitor migration in combination with anti-metastatic drugs ${ }^{109}$. In a tissue-engineering context, micro-fluidic constructions also made it possible to generate growth factor gradients to study the differentiation of neural stem cells 110,111 . In a 3D mesenchymal stem cell culture, growth factor gradients with the most optimal concentration of TGF- $\beta 1$ to induce chondrogenesis were identified ${ }^{112}$.

An emerging technological micro-fluidics application is the use of droplets that enable highthroughput analysis at the single-cell level. The technology is compatible with single-cell omics, which is highlighted by a study with embryonic stem cells where the early onset of differentiation was studied in droplets containing lysis buffer, reaction mix, and barcoded primers ${ }^{113}$. Other applications and techniques concerning droplet-based microfluidics for single-cell omics are excellently reviewed elsewhere ${ }^{114}$.

\section{Small Molecule Screens in Regenerative Medicine}

Libraries of bio-active compounds are a useful high-throughput asset in the field of regenerative medicine to fine-tune growth and differentiation conditions of cultured cells. For instance, compounds targeting pluripotent stem cell viability were identified in a screen utilizing approximately 52,000 compounds ${ }^{115}$, which can be applied to eliminate undifferentiated stem cells before implantation. For directing cell fate, high-throughput screens are a useful tool to identify novel compounds for stem cell differentiation or phenotypical maintenance. For example, in a screen utilizing 4000 compounds, two small molecules were identified that allowed $80 \%$ of ESCs to differentiate towards the endoderm lineage, superior to the commonly used proteins Activin A or Nodal ${ }^{116}$. Also, ESC neuronal differentiation is inducible through small molecules, as shown in a study employing over 100,000 compounds ${ }^{117}$. For MSCs, highthroughput compound screening can be useful to identify molecules that improve MSC differentiation potential. In a study with a library of 1280 small molecules, novel compounds were found that improved the osteogenic differentiation of MSCs with equal efficiency as reference osteogenic molecules such as dexamethasone, vitamin D3, and cAMP ${ }^{118}$. In standard conditions, ESCs are cultured on a fibroblast feeder layer and in the presence of serum. The presence of xenogenic compounds limits clinical application due to potential immunological host reaction, which is why researchers try to define a chemically-defined media composition. In this context, a high-throughput screen with 50,000 compounds resulted in a culture of pluripotent ESCs without a feeder layer, serum, and LIF ${ }^{119}$.

Besides directing cell fate, small molecules are also useful to improve matrix deposition. Through a screen of 1280 small molecules, compounds were identified that improved the 
development of a collagenous-rich matrix in the pre-hypertrophic chondrogenic cell line ATDC5 ${ }^{120}$. These small molecules offer translational perspectives for bone tissue engineering by utilizing them in allografts for promoting endochondral ossification. Through applying the same library, compounds were identified that mimic hypoxia, resulting in increased secretion of VEGF in MSCs ${ }^{121}$. These compounds can have useful tissue engineering applications because VEGF secretion stimulates blood vessel formation.

\section{The Screening Toolbox}

The use of materials to support missing or diseased tissues is not a novel concept. Evidence suggests that the ancient Mayans used nacre shells as a biomaterial to replace missing teeth ${ }^{122}$. Similarly, archeologists found clues that inhabitants of the ancient Roman Empire applied iron as tooth replacement ${ }^{123}$. Other evidence exists that in ancient Egypt inhabitants carried prostheses ${ }^{124}$. In present times, biomaterials are very important for a large group of patients, where titanium hip replacements, stents for obstructed blood vessels, or hemodialysis devices for blood purification can be regarded as significant achievements in the field. In the previous section, we discussed how the properties of materials could be altered to control their bioactivity. Next, we will discuss the technologies needed to engineer these materials.

\section{Micro- and Nanofabrication of Surface Architectures}

\section{Lithography and Hot Embossing}

Multiple methodologies exist to fabricate micro- and nanopatterns. In Figure 5, a schematic overview illustrates the methods we used to construct micro-topographies of the TopoChip platform 47,125 , which is a good example of the different steps to come from design to the final material. First, a mask is produced based on a design, which is subsequently used to produce a mold using the technique of lithography. It involves the use of a sturdy and hard substrate such as silicon or nickel, coating with a photoresist, and shining light through the mask. This mask design corresponds with the topographical pattern, with the passing of UV light subsequently dissolving the underlying photoresist. Next, acid etches the material while leaving the photoresist intact. The duration of the etching step determines the height of the surface structures. After the creation of the mold, the patterns are transferred to a new material that is either photo- or heatcurable. A commonly applied material is the heat-curable polymer polydimethylsiloxane (PDMS) that requires a chemical initiator to harden over time. Typically, PDMS with the initiator is poured over the master mold and fills the pattern cavities. After hardening, the PDMS is peeled off, containing the replica patterns. PDMS can be used directly for cell-based experiments ${ }^{126}$ and is a favorite tool in the microfluidics research field due to its high fidelity for pattern transfer and excellent optical properties. Nevertheless, PDMS is chemically very different from polystyrene, the golden standard for cell culture, which makes it difficult to compare research results ${ }^{127}$. Therefore, patterns can further be transferred into secondary and even tertiary substrates, eventually also into polystyrene. 
Another popular method to transfer imprints into another material is through hot embossing. Here, the material temperature is increased beyond the glass transition temperature, causing it to soften. By applying pressure, patterns are transferred from the mold to the polymer. After cooling, the material is peeled off the mold. Hot embossing is not compatible with every polymer. For instance, direct embossing of polystyrene on a glass waver results in breaking or deforming either the waver or PS due to high demolding forces ${ }^{125}$. It is possible to directly apply hot embossing on a PDMS substrate ${ }^{128}$. However, the pressure might damage the PDMS imprints in the long term. Therefore, as shown in the scheme, we used an intermediate between PDMS and PS, called Ormamold, to transfer the imprints. Here, the Ormostamp is photo-cured through UV radiation, polymerizing the monomers into a hard substrate that carries the negative imprint. The same principles also allow the transfer of nano-patterns with high fidelity ${ }^{52}$. Besides PS, many materials are compatible with hot embossing, making this a highly versatile method for fabricating surfaces, also in an industrial setting ${ }^{129}$. The photo-curing concept to generate Ormostamps can also be applied in a wide variety of tissue-engineering applications. The technique can be harnessed for tooth replacement materials ${ }^{130}$, and cell culture applications such as the generation of scaffolds ${ }^{131}$. Also, for hydrogels, photocuring is very popular due to the possibility to regulate physical behavior dynamically. We refer the reader to a recent review that provides a detailed description of these applications ${ }^{132}$.

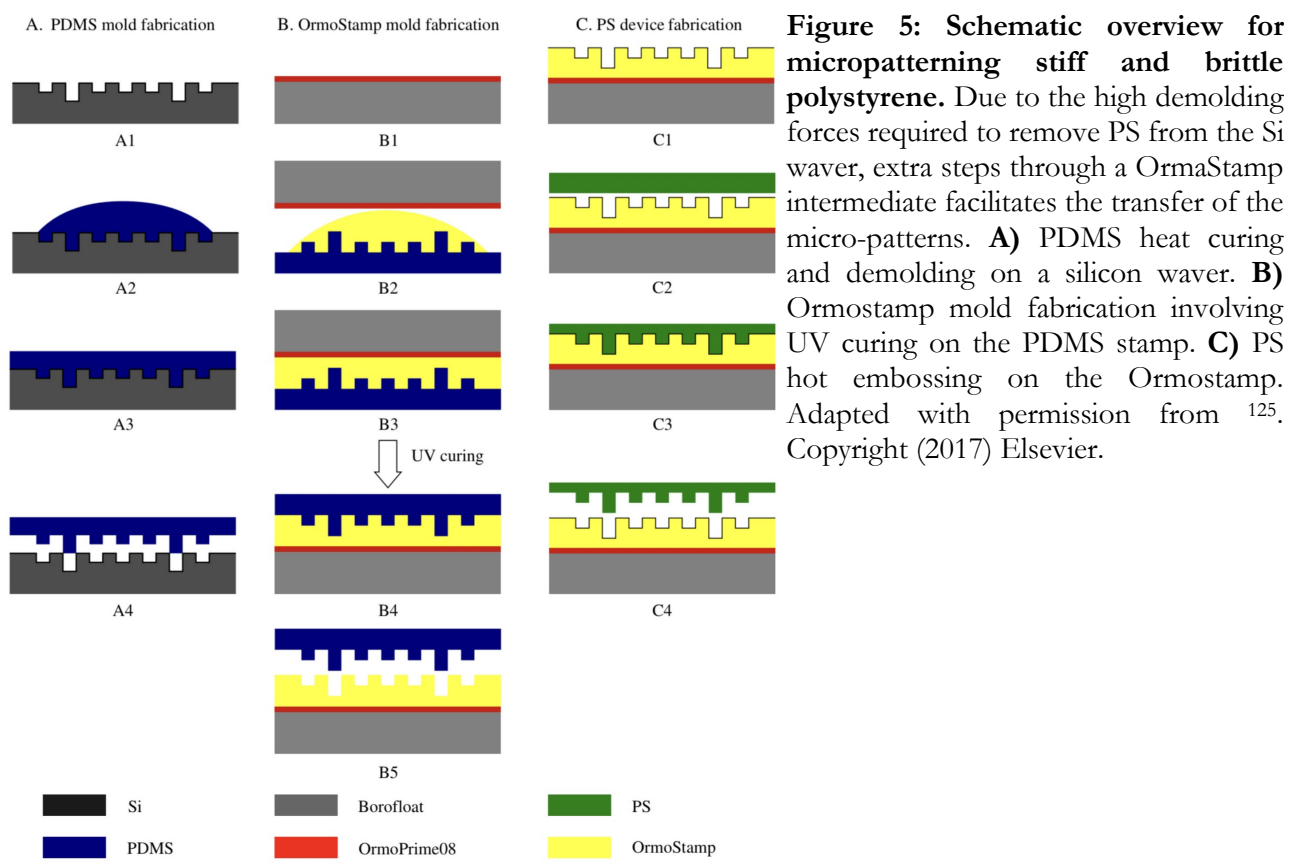


Besides hot embossing, other specialized thermoforming techniques exist. For example, for creating COC microwells inside a screening plate, pressure through an inert gas is applied in combination with heating of the material ${ }^{133}$. Another alternative is injection molding, whereby heated material is directly injected in a mold. This technique finds applications in creating biomaterials such as micro-fluidic platforms ${ }^{134}$. A detailed review of the advantages of these methods are reviewed elsewhere ${ }^{135}$.

\section{Protein and Chemical Patterning}

\section{Microcontact Printing}

Proteins or polymers can be printed onto surfaces in any desired pattern. A commonly applied method to accomplish this is through micro-contact printing. As we explained previously, through lithography techniques, a PDMS stamp with surface structures can be generated. Such a stamp is employed for transferring either matrix proteins or chemicals to a new substrate. Patterns are thus generated when only the elevated PDMS regions come into contact with the substrate. Pattern transfer can be accomplished directly, for example, by coating a PDMS substrate with laminin, after which the laminin is transferred to another substrate by bringing the substrate into contact with PDMS. Such an approach allowed the generation of adhesive islands and subsequent cell patterning in a microfluidic chamber ${ }^{136}$. A more indirect, yet commonly applied strategy, is creating hydrophobic and hydrophilic regions that either allow or disallow protein adsorption. A chemical used to produce these regions can be alkanethiol, which forms self-assembled monolayers (SAMs) on gold ${ }^{137}$. Alkanethiols transfer to the PDMS stamp when dipped into a solution. When the PDMS stamp containing dried alkanethiol comes into contact with a gold substrate, SAMs on this gold substrate will form according to the pattern present on the PDSM stamp. After exposure to a second type of alkanethiol, new SAMs will form between the first SAM patterns. Because end groups on the alkanethiols determine their protein adsorption affinity, patterns of adhesive and non-adhesive regions are created. For instance, methyl groups favor the attachment of proteins, while SAMs with ethylene glycol resist protein adsorption. We provide the reader with a schematic representation in Figure 6. Deviations in these chemistries are common, yet the same principle concerning the use of a stamp is applied. This is demonstrated in a study documenting improved keratinocyte differentiation through adhesive islands ${ }^{62}$. A substantial advantage of the technique is that it allows creating polymer patterns at sub-100 nm resolution ${ }^{138}$. Furthermore, considerable flexibility exists in the viscosity and type of ink chemistry used in the setup, which typically already contains polymers through a mixture of monomers and initiators. For example, lightemitting diodes can be created by stamping a conductive polymer solution ${ }^{139}$. Even the construction of topographical structures is possible through a combination of polymers and inorganic chemistry ${ }^{140,141}$. For a detailed documentation of the wide variety of micro-contact printing applications, we refer the reader to specialized reviews ${ }^{142-144}$. 


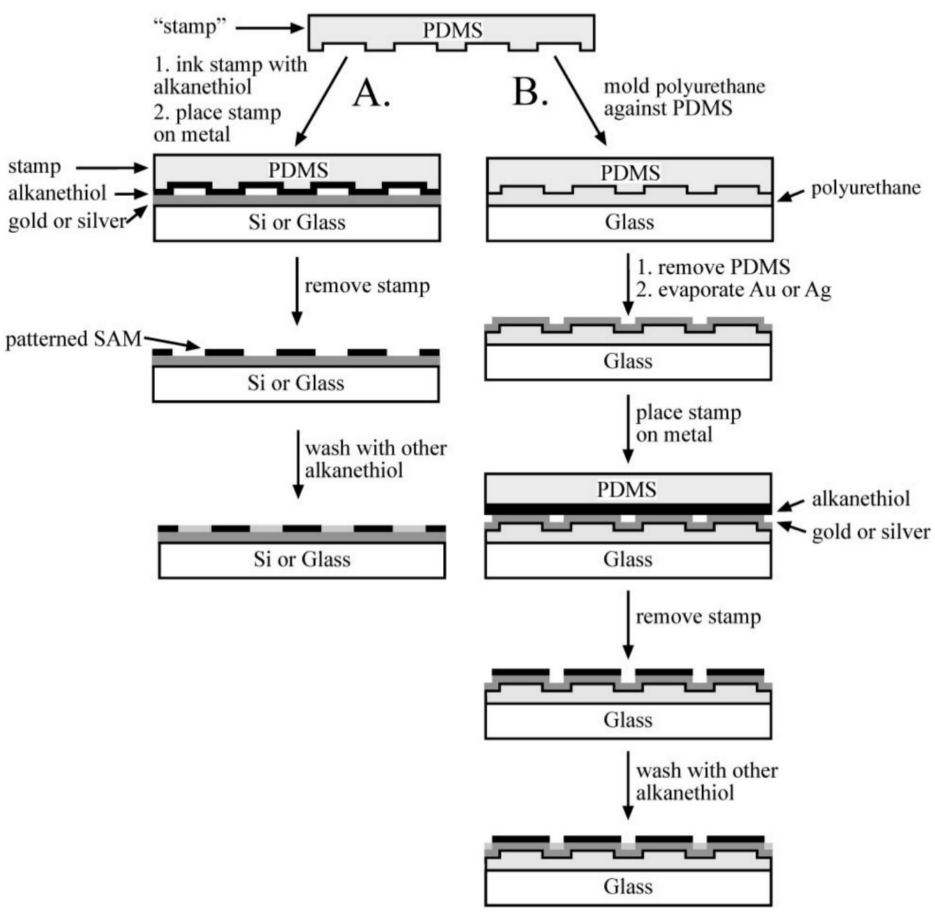

Figure 6: Schematic overview for micropatterning proteins. A) A stamp is inked with an alkanethiol and placed on a gold surface; the pattern on the stamp is transferred to the gold by the formation of an SAM on the regions that contacted the substrate. The bare areas of the gold are exposed to a different alkanethiol to generate a surface patterned with a SAM that presents different chemical functionalities in different regions. B) The PDMS stamp can also be used as a master to mold harder polymers and generate contoured surfaces. After evaporation of a layer of gold, these surfaces can be functionalized by $\mu \mathrm{CP}$ of one alkanethiol with a flat stamp. The grooves of the substrate can then be exposed to an alkanethiol presenting a different functional group to produce a contoured surface with patterned chemical reactivity. Adapted with permission from ${ }^{137}$. Copyright (1999) Elsevier.

\section{Inkjet Printing}

A disadvantage of microcontact printing is that it requires the fabrication of a lithography mask to construct the patters of the stamp. This is time-consuming and also reduces flexibility if new patterns need to be generated. An attractive alternative in this regard is the use of inkjet printers. Here, primary monomers are typically first printed, after which a second monomer or initiator is mixed with the first monomer, allowing in situ polymerization of the monomers ${ }^{145}$. The technique, therefore, not only allows flexibility in pattern design, yet can also create a broad polymer diversity making this ideal for high-throughput studies. For example, inkjet printing allowed designing 2000 different hydrogels, of which their functionality was analyzed for cell adhesion and thermos-responsive release ${ }^{100}$, and for screening 380 different polyurethanes and acrylates for inducing a hepatocyte phenotype ${ }^{86}$. Deviations usually exist by altering the surrounding environment during the printing process. Polymerization reactions can be inhibited when oxygen is present, or slow down the polymerization reactions, causing the formation of 
irregular spots. These considerations were taken into account in studies utilizing the printing of hundreds of polymer spots for ESC differentiation ${ }^{83}$ and antibacterial attachment ${ }^{89}$ by utilizing an argon atmosphere and a UV lamp to increase the polymerization speed. A disadvantage compared to microcontact printing are restrictions in the physical parameters of the chemical, such as high viscosity levels ${ }^{146}$.

\section{Photopatterning}

Lithography can directly pattern polymer surfaces, a method that does not require etching of a mold. For example, exposure of surface-bound benzophenone to UV light enables stable binding with target molecules ${ }^{147}$. Through this method, matrix proteins are covalently bound to the surface for cell adhesion experiments. Previously, we have shown that the use of photoresists is useful for micro-patterning, and the same principle can also be utilized for protein patterning when the resist adsorbs proteins. Photomasks give precise control of the desired pattern and are also applicable for high-throughput purposes without the need to fabricate a stamp such as in microcontact printing ${ }^{148}$.

\section{Computational Techniques}

High-throughput experiments are characterized by generating massive amounts of data. To mine this wealth of data, know-how is needed to process data correctly. In this time of big data, the need to handle it increases, and fortunately, more and more user-friendly bioinformatics tools exist to process them. Computer languages such as $\mathrm{R}^{149}$ and Python ${ }^{150}$ are becoming more accessible, thanks to interfaces such as R Studio 151 and Spyder. Also, numerous online learning platforms such as Edx (https://www.edx.org), Coursera (https://www.coursera.org), and DataCamp (https://www.datacamp.com) offer basic and advanced bioinformatics courses. For high-throughput imaging experiments, which can quickly amount to thousands of images, userfriendly software such as Cellprofiler and Fuji exist for handling large quantities ${ }^{152,153}$. Machine learning algorithms to correlate the experimental design variables to phenotypical outcomes can be handled by software such as Rapidminer ${ }^{154}$. In this chapter, we provide the reader with an overview and description of how these computational techniques facilitate the analysis of highthroughput data.

\section{Batch Effects}

Data derived from high-throughput technologies is prone to batch effects due to data retrieval from multiple time points or different experimental conditions. This leads to technical variations in the data, resulting in biases and misleading conclusions. Batch effects can occur through the performance of experiments by multiple operators, the use of different laboratory equipment, and variations in serum or growth factor batches and other reagents. Biological factors can also play a role. For example, the use of different stem cell donors can influence experimental outcomes, as illustrated by Siddappa et al., demonstrating that the differentiation potential of MSCs can vary between donors ${ }^{155}$. Although batch effects can occur in any experimental setup, finding batch effects is easier for high-throughput and high-dimensional data. Examples of the 
identification of batch effects in scientific studies are found in an interesting review by Leek $e t$ al., where methods are described to correctly handle data influenced by batch effects ${ }^{156}$. An example of such a method is surrogate variable analysis, which demonstrated its usefulness for overcoming heterogeneity in gene expression studies ${ }^{157}$. Also, batch effects in high-throughput applications can be identified by machine learning techniques like clustering analysis and PCA, which will be discussed further.

\section{Image Processing}

Imaging is a particularly suitable technique for obtaining high-throughput data and is facilitated by microscopes that can take thousands of images in a matter of hours. Our group applied this technique multiple times on the TopoChip platform that contains a total of 4352 topographical units on a $2 \mathrm{~cm}^{2}$ surface ${ }^{49,51,158}$. A limitation is that usually, only a few proteins are targeted due to the spectral overlap of the applied fluorochromes. However, for improving the information content of images, techniques such as CellPaint allow staining multiple cell organelles besides the classical nuclear or actin staining ${ }^{159}$. To analyze the large number of images that are typically generated, software scripts such as in Matlab can identify and quantify both cell morphology and intensity features of the images ${ }^{47}$. However, also open access software such as CellProfiler allows image processing both in 2D and 3D while enabling the extraction of highly dimensional data ${ }^{160}$. Examples of applications include the segmentation of nuclei and quantifying DNA content in an RNAi library screen ${ }^{161}$, or measuring protein levels in both the cytoplasm and the nucleus during differentiation events ${ }^{162}$. Also, in immunohistochemistry images, CellProfiler identified CD4+ and CD8+ T cells in human tissue samples ${ }^{163}$ and quantified the amount of CD4+ cells in patients with chronic graft-versus-host disease for determining disease severity ${ }^{164}$.

The algorithms that allow pattern recognition are continuously improved. A promising and emerging strategy in this regard is the use of deep learning algorithms. This machine learning approach allows superior nuclei segmentation across multiple image types ${ }^{165,166}$. Furthermore, it can be applied in a high-throughput context for identifying cellular phenotypes ${ }^{167}$. In the clinic, deep learning algorithms are beneficial for the automated detection of acute lymphoblastic leukemia cells ${ }^{168}$, tumors ${ }^{169}$, and polyps ${ }^{170}$. Since the creation of these scripts requires a specialist to develop them, the application by biologists and clinicians is typically performed through software such as U-Net ${ }^{171}$. CellProfiler also offers modules for applying pre-trained neural network models for cell segmentation ${ }^{172}$, assessing the quality of images ${ }^{173}$, and tracking cells during live-cell imaging ${ }^{174}$. In the future, more of these algorithms will become available, which, in combination with user-friendly software, will further increase the possibilities and quality of image-based studies. We further refer to two recent reviews that describe in detail the use of deep learning for cellular imaging ${ }^{175}$ and medical image analysis ${ }^{176}$. 


\section{Machine Learning}

Besides applying deep learning in imaging studies, also other machine learning algorithms are useful in a high-throughput context. For example, when we would like to understand which and how strong experimental variables contribute to an observed effect. This effect can be a phenotypic condition, with the input parameters being a wide variety of different materials or polymers. Through machine learning, meaningful relationships between input and output variables can be identified and visualized with the possibility to predict outcomes. Furthermore, machine learning approaches such as PCA allows dimensionality reduction, thereby enabling to filter out irrelevant data from datasets. In this section, we describe how machine learning algorithms benefit high-throughput data analysis. We also refer to a recent review from our group on the use of machine learning algorithms in a biomaterial context ${ }^{177}$.

\section{Regression Analysis}

A classical machine learning approach for measuring cell-material interactions is through linear regression. This method employs a best-fit line whereby the distance between the actual and predicted measurements is minimized. This allows determining and quantifying significant correlations between the input (independent) and output (dependent) variables enabling the prediction of outcomes (Figure 7A). More complex methodologies exist, such as multivariate linear regression with more than one independent variable. The following examples show how regression models are useful for regenerative medicine applications. A regression method was used for inferring if the output from magnetic resonance imaging correlates with glycosaminoglycan content of different engineered cartilage constructs ${ }^{178}$. Through multivariate linear regression, optimal polyurethane compositions were identified against microbial attachment ${ }^{179}$. Similarly, regression models allowed identifying the effect of antibacterial attachment of ions in a polymer array ${ }^{89}$.

\section{Classifier Algorithms}

For some applications, it might be important to classify observations according to specific criteria. This is useful for determining if an email should be classified as spam, or for identifying plant species based on a picture alone ${ }^{180}$. Here, classifier algorithms are trained for determining which characteristics correspond best with a specific category. Usually, two subsections of the data are taken, one for training the classifications and one for evaluating the performance of the classifier. Many classifier algorithms exist; these include k-nearest neighbors ${ }^{181}$, random forests ${ }^{182}$, support vector machines (SVM) ${ }^{183}$, and the before mentioned deep learning ${ }^{184}$. These algorithms are particularly well suited for high-throughput applications. For example, besides useful for image processing, deep learning classifiers allowed modeling and predicting which polymer characteristics are associated with embryoid body cell adhesion ${ }^{185}$. In our group, we applied random forest algorithms for identifying which topographical features are associated with a particular phenotype (Figure 7B) ${ }^{48,50,51}$. 


\section{Cluster Methods and Dimensionality Reduction}

For understanding and visualizing complex data acquired from high-throughput experiments, clustering analysis is an essential computational approach. This method groups data points based on similarity and is therefore useful for identifying surface structures or polymers with similar or unique effects on cell behavior. An example of such a cluster method is called k-means clustering 186. $\mathrm{k}$-means is a relatively simple algorithm adapted in many scientific disciplines. Applications include grouping differentially expressed genes together to allow visualizing and identifying genes associated with the different developmental stages of the kidney ${ }^{187}$. Similarly, in embryonic stem cells and trophoblasts, genes associated with pluripotency and lineage specificity can be clustered and distinguished ${ }^{188}$. However, k-means suffers from some drawbacks, since the user has to choose the number of clusters beforehand, leading towards potential biases. Furthermore, highly dimensional data is challenging to analyze through the k-means method as well as for similar clustering approaches such as fuzzy c-means ${ }^{189}$.

An alternative is hierarchical clustering. Here, all data points are split into two clusters that share the least similarity based on Euclidian difference. This process is repeated until each data point is divided into a cluster, creating a tree-like graph. We previously mentioned the TopoWellPlate, where the influence of a large variety of surface structures on the secretome of MSCs was investigated ${ }^{54}$. Through hierarchical clustering, we grouped surfaces from the TopoWell plate that exert similar or unique effects on the secretome profile of MSCs. In a similar approach, yet with polymers instead of surface structures, and microarray data instead of multiplex ELISA, the effect of different polymers on gene expression profiles in MSCs was assessed through clustering ${ }^{190}$. Multiple variations in clustering algorithms exist, of which we refer the reader to a specialized review discussing their advantages and disadvantages ${ }^{191}$.

Datasets that are large in dimensionality can have adverse effects on cluster algorithms ${ }^{192}$. This is illustrated in a study where miRNA levels were measured from multiple cancer cell lines. When 217 miRNA targets (dimensionality of 217) were taken into account, hierarchical clustering allowed to cluster cell lines from the colon, liver, pancreas, and stomach origin together, reflecting their endodermic origin (Figure 7C) ${ }^{193}$. However, this relationship was lost when 16000 mRNA's were included in the analysis due to higher dimensionality.

Not all variables that contribute to higher dimensionality levels are relevant and thereby create noise in cluster algorithms. A method to overcome this is through principal component analysis (PCA) ${ }^{194}$, which reduces data dimensionality. Besides a useful tool to enhance the accuracy of clustering methods, it is also frequently applied to assess visual similarities between data points. PCA simplifies dimensional complexity by geometrically projecting the data into a lowerdimensional space. This method was applied to demonstrate that a flat tissue culture surface, a flat polyimide surface, and a nanogrooved polyimide surface, induced distinct gene expression profiles independent of MSC donor variability ${ }^{26}$. In research where the effect of microtopographies on chondrogenesis was assessed, PCA distinguished surfaces based on the gene expression and morphological profiles they elicit ${ }^{195}$. In a cytokine screening of multiple cancer 
cell lines cultured on different hydrogels, PCA allowed identifying biomaterials that induced similar or distinct cytokine profiles (Figure 7D) ${ }^{196}$.

PCA can, in some cases, experience computational limitations, which disallows a meaningful data visualization ${ }^{197}$. An alternative technique that is gaining popularity for dimensionality reduction and visualization of high-dimensional datasets is t-distributed stochastic neighbor embedding (t-SNE) ${ }^{198}$. This method visualized different human tissues based on their respective gene expression levels ${ }^{199}$. Similarly, dynamic developmental processes in the zebrafish embryo were visualized through single-cell RNA sequencing of more than 92000 cells ${ }^{200}$. In the future, this technique will likely gain traction in high-throughput applications with highly dimensional data-sets. All the previous examples demonstrate that machine-learning algorithms are useful tools for a correct data interpretation of high-throughput applications. 
A

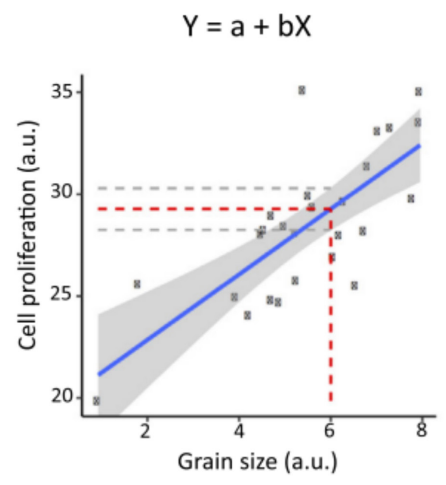

C

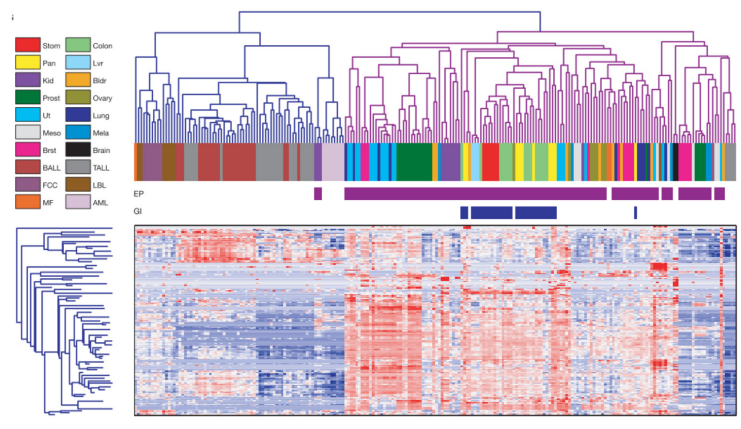

B



D

Figure 7: Machine learning examples applied in regenerative medicine research. A) Simple linear regression example. The dependent variable $(\mathrm{Y})$ is predicted using the independent variable $(\mathrm{X})$. Here, the output (cell proliferation) is the dependent variable, and the input (grain size) is the independent variable. Adapted with permission from ${ }^{177}$. Copyright (2017) Elsevier. B) Example of a Random Forest output. Each bar represents the importance of a surface feature characteristic associated with improved phenotypic characteristics in tenocytes. Data originates from a TopoChip screen containing 2176 different microtopographies with Scleraxis, a tenogenic marker, as output. Adapted with permission from ${ }^{51}$. Copyright (2019) Elsevier. C) Hierarchical clustering representation of miRNA profiles from 218 tissue samples. Samples are in columns, miRNA expression in rows. Samples from the same tissue and lineage origin cluster together. Adapted with permission from ${ }^{193}$. Copyright (2015) Springer Nature. D) PCA Dimensionality reduction performed on the secretion profile from 5 different cell lines cultured on 5 different biomaterials. PCA demonstrates that in this setup, alginate and PEG induce a similar secretion profile while being distinct from agarose, collagen, and matrigel. Between cell lines, agarose induces a distinct secretion profile compared to the other cell lines cultured on the same substrate. Adapted with permission from ${ }^{196}$. Copyright (2017) American Chemical Society. 


\section{Discussion}

Throughout this work, we presented multiple high-throughput approaches (Table 1). A general disadvantage of these platforms is that the output only encompasses a few parameters, with ICC as the most prominent technique. This is attributed due to technical limitations. In the highthroughout examples we gave, there are usually insufficient cell numbers to detect meaningful RNA or protein concentrations. Nevertheless, techniques such as the aforementioned CellPaint assay can increase the screening information content ${ }^{159}$. Furthermore, other techniques such as mass-spectrometric imaging ${ }^{201}$, and single-cell RNA sequencing, might in the future find useful high-throughput applications. When reducing the total number of input variables towards a wellplate format, other techniques such as qPCR, ELISA, RNA sequencing, and proteomics become possible due to the presence of sufficient cell numbers ${ }^{35,54}$. However, a strategy that is commonly employed is first to find positive hits through a high-throughput screen. Afterwards, the positive hits are fabricated in a larger format, enabling broader investigations into other markers or pathways through other techniques besides ICC ${ }^{51}$.

Screening technologies brought new culture environments that are useful for regenerative medicine, while in parallel provided us with novel biological insights. However, the enormous complexity that exists also illustrates the challenges the field faces. Although high-throughput platforms comprise many perturbations, these are usually focused on one aspect of the cell niche. For example, different roughness levels are rarely combined with various geometric shapes, surface chemistry, and culture media compositions. Likewise, high-throughput screens that investigate multiple matrix compositions do not include surface structures or apply stretch forces. This can result in the loss of valuable information. For example, mechanical stimulation can upregulate the presence of growth factor receptors in smooth muscle cells, sensitizing them against growth factor stimulation ${ }^{202}$. Integrins that bind to specific matrix molecules influence growth factor activation by forming complexes with growth factor receptors 203,204. The consequence of this can be that high-throughput screens utilizing growth factors or small molecules in a collagen substrate environment can elicit different cell behavior compared to a laminin substrate. Also, surface structures can alter cellular sensitivity against growth factors ${ }^{205}$, and the biological activity of matrix compounds ${ }^{206}$. These are only a few examples of how combinatorial environments can further improve experimental outcomes and underlines the importance of employing this principle in high-throughput screens, which is still an underdeveloped concept. The main reasons for these sparse combinatorial approaches are either technical challenges or the lack of know-how.

Another interesting viewpoint is the use of databases to supplement and support data derived from high-throughput applications. Techniques such as Cell Paint that produce a morphological fingerprint ${ }^{159}$, can further be compared with databases of other fingerprints produced by gene alterations or small molecule treatment ${ }^{207,208}$. In line with this are the possibilities that large scale gene expression profiling initiatives offer, such as the recent expansion of the Connectivity Map 209, which contains a million gene signatures of cell lines treated with small molecules, shRNA, 
and overexpression constructs. With the rise in automatization, omics technologies, and artificial intelligence, we foresee big opportunities to speed up scientific discoveries ${ }^{210}$. In the future, cross-high-throughput platforms involving design, chemistry, and biochemistry combined with high-content technologies will open the road to novel biological insights and regenerative medicine applications.

\begin{tabular}{|c|c|c|c|c|}
\hline Screening platform & $\begin{array}{l}\text { Essential } \\
\text { techniques }\end{array}$ & $\begin{array}{l}\text { Maximal } \\
\text { described sample } \\
\text { size }\end{array}$ & Biological assays & References \\
\hline Roughness & Etching & Gradients & ICC & {$[13],[18]$} \\
\hline Surface structures & $\begin{array}{l}\text { Lithography - } \\
\text { hot embossing }\end{array}$ & $\begin{array}{l}2176 \\
\text { Well format (96- } \\
384 \text { samples) }\end{array}$ & $\begin{array}{l}\text { ICC } \\
\text { ICC - } \\
\text { Genomics/Proteomics } \\
\text { assays }\end{array}$ & $\begin{array}{l}{[26],[38],[42],[51],[52]} \\
{[35],[54]}\end{array}$ \\
\hline $\begin{array}{l}\text { Protein/polymer } \\
\text { patterns }\end{array}$ & $\begin{array}{l}\text { Microcontact } \\
\text { printing }\end{array}$ & (1) & ICC & {$[60],[61],[62],[136]$} \\
\hline & Photo patterning & (1) & ICC & {$[147],[148]$} \\
\hline $\begin{array}{l}\text { Polymers/hydrogels } \\
\text { compositions }\end{array}$ & Inkjet printing & 2280 & ICC & {$[83],[86],[89],[100]$} \\
\hline $\begin{array}{l}\text { Small molecule } \\
\text { screenings }\end{array}$ & $\begin{array}{l}\text { Robotics for } \\
\text { large sample size }\end{array}$ & 100,000 & $\begin{array}{l}\text { ICC - } \\
\text { Genomics/Proteomics } \\
\text { assays }\end{array}$ & $\begin{array}{l}{[115],[116],[117],} \\
{[118],[119],[120],[121]}\end{array}$ \\
\hline
\end{tabular}

Table 1: Summary of different high-throughput approaches. (1) Not applied in a high-throughput context. However, depending on the layout of the lithography mask, this can be accomplished. For example, by applying the lithography mask of the TopoChip platform.

\section{Acknowledgments}

SV acknowledges the financial support of the European Union's Horizon 2020 Programme (H2020-MSCA-ITN-2015; Grant agreement 676338). We gratefully thank Urnaa Tuvshindorj for sharing a figure of a RGD adhesive island based high-throughput platform.

\section{References}

1. Sharma, R. et al. Clinical-grade stem cell-derived retinal pigment epithelium patch rescues retinal degeneration in rodents and pigs. Sci. Transl. Med. 11, 1-14 (2019).

2. Cionca, N., Müller, N. \& Mombelli, A. Two-piece zirconia implants supporting all-ceramic crowns: A prospective clinical study. Clin. Oral Implants Res. 26, 413-418 (2015).

3. Nicolle, L. E. Catheter associated urinary tract infections. Antimicrob. Resist. Infect. Control 3, 1-8 (2014).

4. Anderson, J. M., Rodriguez, A. \& Chang, D. T. Foreign body reaction to biomaterials. Semin. Immunol. 20, 86-100 (2008). 
5. Victor, J. et al. Total knee arthroplasty at 15-17 years: Does implant design affect outcome? Int. Orthop. 38, 235-241 (2014).

6. Holmich, L. R. et al. Incidence of Silicone Breast Implant Rupture. Arch Surg. 138, 801-806 (2003).

7. Groen, N. et al. Linking the Transcriptional Landscape of Bone Induction to Biomaterial Design Parameters. Adv. Mater. 29, (2017).

8. Tare, R. S. et al. A microarray approach to the identification of polyurethanes for the isolation of human skeletal progenitor cells and augmentation of skeletal cell growth. Biomaterials 30, 1045-1055 (2009).

9. Holtzer, H., Abbot, J., Lash, J. \& S., H. The Loss of Phenotypic Traits by Differentiated Cells in vitro, I. Dedifferentiation of Cartilage Cells. PNAS 46, 1533-1542 (1960).

10. Buser, D. et al. Influence of surface characteristics on bone integration of titanium implants. A histomorphometric study in miniature pigs. J. Biomed. Mater. Res. 25, 889-902 (1991).

11. Buser, D. et al. Enhanced Bone Apposition to a Chemically Modified SLA Titanium Surface. J Dent Res 83, 529-533 (2004).

12. Martin, J. Y. et al. Effect of titanium surface roughness on proliferation, differentiation, and protein synthesis of human osteoblast-like cells (MG63). J. Biomed. Mater. Res. 29, 389-401 (1995).

13. Faia-Torres, A. B. et al. Differential regulation of osteogenic differentiation of stem cells on surface roughness gradients. Biomaterials 35, 9023-9032 (2014).

14. Müller, P. et al. Calcium phosphate surfaces promote osteogenic differentiation of mesenchymal stem cells. J. Cell. Mol. Med. 12, 281-291 (2008).

15. Jaggy, M. et al. Hierarchical Micro-Nano Surface Topography Promotes Long-Term Maintenance of Undifferentiated Mouse Embryonic Stem Cells. Nano Lett. 15, 7146-7154 (2015).

16. Jeon, K. et al. Self-renewal of embryonic stem cells through culture on nanopattern polydimethylsiloxane substrate. Biomaterials 33, 5206-5220 (2012).

17. Chung, T. W., Liu, D. Z., Wang, S. Y. \& Wang, S. S. Enhancement of the growth of human endothelial cells by surface roughness at nanometer scale. Biomaterials 24, 4655-4661 (2003).

18. Kunzler, T. P., Drobek, T., Schuler, M. \& Spencer, N. D. Systematic study of osteoblast and fibroblast response to roughness by means of surface-morphology gradients. Biomaterials 28, 2175-2182 (2007).

19. Werner, M. et al. Surface Curvature Differentially Regulates Stem Cell Migration and Differentiation via Altered Attachment Morphology and Nuclear Deformation. Adv. Sci. 4, 1-11 (2017).

20. Werner, M., Kurniawan, N. A., Korus, G., Bouten, C. V. C. \& Petersen, A. Mesoscale substrate curvature overrules nanoscale contact guidance to direct bone marrow stromal cell migration. J. R. Soc. Interface 15, (2018).

21. Werner, M., Petersen, A., Kurniawan, N. A. \& Bouten, C. V. C. Cell-Perceived Substrate Curvature Dynamically Coordinates the Direction, Speed, and Persistence of Stromal Cell Migration. Adv. Biosyst. 3, (2019).

22. Baptista, D., Teixeira, L., van Blitterswijk, C., Giselbrecht, S. \& Truckenmüller, R. Overlooked? Underestimated? Effects of Substrate Curvature on Cell Behavior. Trends Biotechnol. 37, 838-854 (2019).

23. Zhou, Q. et al. Directional nanotopographic gradients: A high-throughput screening platform for cell contact guidance. Sci. Rep. 5, 1-12 (2015).

24. Boon, T. A. B. Van Der, Yang, L., Li, L. \& Galván, D. E. C. Well Plate Integrated Topography Gradient Screening Technology for Studying Cell-Surface Topography Interactions. Adv. Biosyst. 1900218, (2019).

25. Zhou, Q. et al. Screening Platform for Cell Contact Guidance Based on Inorganic Biomaterial Micro/nanotopographical Gradients. ACS Appl. Mater. Interfaces 9, 31433-31445 (2017).

26. Abagnale, G. et al. Surface topography enhances differentiation of mesenchymal stem cells towards osteogenic and adipogenic lineages. Biomaterials 61, 316-326 (2015).

27. López-Bosque, M. J. et al. Fabrication of hierarchical micro-nanotopographies for cell attachment studies. Nanotechnology 24, (2013).

28. Downing, T. L. et al. Biophysical regulation of epigenetic state and cell reprogramming. Nat. Mater. 12, 1154-1162 (2013).

29. Abagnale, G. et al. Surface Topography Guides Morphology and Spatial Patterning of Induced Pluripotent Stem Cell Colonies. Stem Cell Reports 9, 654-666 (2017). 
30. Lee, M. R. et al. Direct differentiation of human embryonic stem cells into selective neurons on nanoscale ridge/groove pattern arrays. Biomaterials 31, 4360-4366 (2010).

31. Mattotti, M. et al. Inducing functional radial glia-like progenitors from cortical astrocyte cultures using micropatterned PMMA. Biomaterials 33, 1759-1770 (2012).

32. English, A. et al. Substrate topography: A valuable in vitro tool, but a clinical red herring for in vivo tenogenesis. Acta Biomater. 27, 3-12 (2015).

33. Zhu, J. et al. The regulation of phenotype of cultured tenocytes by microgrooved surface structure. Biomaterials 31, 6952-6958 (2010).

34. Mattotti, M. et al. Differential neuronal and glial behavior on flat and micro patterned chitosan films. Colloids Surfaces B Biointerfaces 158, 569-577 (2017).

35. Hu, J. et al. High-Throughput Mechanobiology Screening Platform Using Micro- and Nanotopography. Nano Lett. 55, 9557-9561 (2017).

36. Moe, A. A. K. et al. Microarray with micro- and nano-topographies enables identification of the optimal topography for directing the differentiation of primary murine neural progenitor cells. Small 8 , 3050-3061 (2012).

37. Ankam, S. et al. Substrate topography and size determine the fate of human embryonic stem cells to neuronal or glial lineage. Acta Biomater. 9, 4535-4545 (2013).

38. Tan, K. K. B. et al. Enhanced differentiation of neural progenitor cells into neurons of the mesencephalic dopaminergic subtype on topographical patterns. Biomaterials 43, 32-43 (2015).

39. Dalby, M. J. et al. The control of human mesenchymal cell differentiation using nanoscale symmetry and disorder. Nat. Mater. 6, 997-1003 (2007).

40. Rasmussen, C. H. et al. Enhanced Differentiation of Human Embryonic Stem Cells Toward Definitive Endoderm on Ultrahigh Aspect Ratio Nanopillars. Adv. Funct. Mater. 26, 815-823 (2016).

41. Kong, Y. P., Tu, C. H., Donovan, P. J. \& Yee, A. F. Expression of Oct4 in human embryonic stem cells is dependent on nanotopographical configuration. Acta Biomater. 9, 6369-6380 (2013).

42. Lovmand, J. et al. The use of combinatorial topographical libraries for the screening of enhanced osteogenic expression and mineralization. Biomaterials 30, 2015-2022 (2009).

43. Kolind, K. et al. Control of proliferation and osteogenic differentiation of human dental-pulpderived stem cells by distinct surface structures. Acta Biomater. 10, 641-650 (2014).

44. Markert, L. D. et al. Identification of Distinct Topographical Surface Microstructures Favoring Either Undifferentiated Expansion or Differentiation of Murine Embryonic Stem Cells. Stem Cells Dev. $18,1331-1342$ (2009).

45. Joergensen, N. L. et al. Topography-Guided Proliferation: Distinct Surface Microtopography Increases Proliferation of Chondrocytes In Vitro. Tissue Eng. Part A 21, 2757-2765 (2015).

46. Kolind, K. et al. A combinatorial screening of human fibroblast responses on micro-structured surfaces. Biomaterials 31, 9182-9191 (2010).

47. Unadkat, H. V et al. An algorithm-based topographical biomaterials library to instruct cell fate. Proc. Natl. Acad. Sci. U. S. A. 108, 16565-70 (2011).

48. Reimer, A. et al. Scalable topographies to support proliferation and Oct4 expression by human induced pluripotent stem cells. Sci. Rep. 6, 18948 (2016).

49. Vasilevich, A. S., Mourcin, F., Mentink, A., Hulshof, F. \& Beijer, N. Designed Surface Topographies Control ICAM-1 Expression in Tonsil-Derived Human Stromal Cells. Front. Bioeng. Biotechnol. 6, 1-14 (2018).

50. Hulshof, F. F. B. et al. Mining for osteogenic surface topographies: In silico design to in vivo osseo-integration. Biomaterials 137, 49-60 (2017).

51. Vermeulen, S. et al. Identification of topographical architectures supporting the phenotype of rat tenocytes. Acta Biomater. 83, 277-290 (2019).

52. Hulshof, F. F. B. et al. NanoTopoChip: High-throughput nanotopographical cell instruction. Acta Biomater. 62, 188-198 (2017).

53. Beijer, N. R. M. et al. TopoWellPlate: A Well-Plate-Based Screening Platform to Study CellSurface Topography Interactions. Adv. Biosyst. 1, 1700002 (2017).

54. Leuning, D. G. et al. The cytokine secretion profile of mesenchymal stromal cells is determined by surface structure of the microenvironment. Sci. Rep. 8, 1-9 (2018).

55. Plow, E. F., Haas, T. A., Zhang, L., Loftus, J. \& Smith, J. W. Ligand binding to integrins. J. Biol. Chem. 275, 21785-21788 (2000). 
56. Jokinen, J. et al. Integrin-mediated cell adhesion to type I collagen fibrils. J. Biol. Chem. 279, 31956-31963 (2004).

57. Belkin, A. M. \& Stepp, M. A. Integrins as receptors for laminins. Microsc. Res. Tech. 51, 280301 (2000).

58. Orlowska, A. et al. The effect of coatings and nerve growth factor on attachment and differentiation of Pheochromocytoma Cells. Materials (Basel). 11, 1-10 (2017).

59. Hayashi, Y. et al. Integrins Regulate Mouse Embryonic Stem Cell Self-Renewal. Stem Cells 25, 3005-3015 (2007).

60. Singhvi, R. et al. Engineering Cell Shape and Function. Science (80-. ). 264, 696-698 (1994).

61. McBeath, R., Pirone, D. M., Nelson, C. M., Bhadriraju, K. \& Chen, C. S. Cell shape, cytoskeletal tension, and RhoA regulate stem cell lineage commitment. Dev. Cell 6, 483-495 (2004).

62. Connelly, J. T. et al. Actin and serum response factor transduce physical cues from the microenvironment to regulate epidermal stem cell fate decisions. Nat. Cell Biol. 12, 711-718 (2010).

63. Flaim, C. J., Chien, S. \& Bhatia, S. N. An extracellular matrix microarray for probing cellular differentiation. Nat. Methods 2, 119-125 (2005).

64. Kwon, S. J., Lee, M., Ku, B., Sherman, D. H. \& Dordick, J. S. Defined Substrates for Human Embryonic Stem Cell Growth Identified from Surface Arrays. ACS Chem. Biol. 2, 419-425 (2007).

65. Soen, Y., Mori, A., Palmer, T. D. \& Brown, P. O. Exploring the regulation of human neural precursor cell differentiation using arrays of signaling microenvironments. Mol. Syst. Biol. 2, 1-14 (2006).

66. Nakajima, M. et al. Combinatorial protein display for the cell-based screening of biomaterials that direct neural stem cell differentiation. Biomaterials 28, 1048-1060 (2007).

67. Neto, A. I., Custódio, C. A., Song, W. \& Mano, J. F. High-throughput evaluation of interactions between biomaterials, proteins and cells using patterned superhydrophobic substrates. Soft Matter 7, 41474151 (2011).

68. Tzoneva, R., Faucheux, N. \& Groth, T. Wettability of substrata controls cell-substrate and cellcell adhesions. Biochim. Biophys. Acta - Gen. Subj. 1770, 1538-1547 (2007).

69. Van Kooten, T. G., Spijker, H. T. \& Busscher, H. J. Plasma-treated polystyrene surfaces: Model surfaces for studying cell-biomaterial interactions. Biomaterials 25, 1735-1747 (2004).

70. Mitchell, S. A. et al. Cellular attachment and spatial control of cells using micro-patterned ultraviolet/Ozone treatment in serum enriched media. Biomaterials 25, 4079-4086 (2004).

71. Mitchell, S. A., Poulsson, A. H. C., Davidson, M. R. \& Bradley, R. H. Orientation and confinement of cells on chemically patterned polystyrene surfaces. Colloids Surfaces B Biointerfaces 46, 108-116 (2005).

72. Poulsson, A. H. C., Mitchell, S. A., Davidson, M. R., Emmison, N. \& Bradley, R. H. Adhesion of primary human osteoblast cells to UV/ozone modified polyethylene. Langmuir 25, 3718-3727 (2009).

73. Grinnell, F. \& Feld, M. K. Fibronectin adsorption on hydrophilic and hydrophobic surfaces detected by antibody binding and analyzed during cell adhesion in serum-containing medium. J. Biol. Chem. 257, 4888-4893 (1982).

74. Keselowsky, B. G., Collard, D. M. \& García, A. J. Surface chemistry modulates fibronectin conformation and directs integrin binding and specificity to control cell adhesion. J. Biomed. Mater. Res. Part A 66A, 247-259 (2003).

75. Urquhart, A. J. et al. High throughput surface characterisation of a combinatorial material library. Adv. Mater. 19, 2486-2491 (2007).

76. Thaburet, J. F., Mizomoto, H. \& Bradley, M. High-Throughput Evaluation of the Wettability of Polymer Libraries. Macromol. Rapid Commun. 25, 366-370 (2004).

77. Weber, N., Bolikal, D., Bourke, S. L. \& Kohn, J. Small changes in the polymer structure influence the adsorption behavior of fibrinogen on polymer surfaces: Validation of a new rapid screening technique. J. Biomed. Mater. Res. - Part A 68, 496-503 (2004).

78. Maitz, M. F. Applications of synthetic polymers in clinical medicine. Biosurface and Biotribology 1, 161-176 (2015).

79. Tourniaire, G. et al. Polymer microarrays for cellular adhesion. Chem. Commun. 2118-2120 (2006). doi:10.1039/b602009g

80. Anderson, D. G., Putnam, D., Lavik, E. B., Mahmood, T. A. \& Langer, R. Biomaterial microarrays: Rapid, microscale screening of polymer-cell interaction. Biomaterials 26, 4892-4897 (2005). 
81. Pernagallo, S., Diaz-Mochon, J. J. \& Bradley, M. A cooperative polymer-DNA microarray approach to biomaterial investigation. Lab Chip 9, 397-403 (2009).

82. Treiser, M. D. et al. Cytoskeleton-based forecasting of stem cell lineage fates. Proc. Natl. Acad. Sci. U. S. A. 107, 610-615 (2010).

83. Anderson, D. G., Levenberg, S. \& Langer, R. Nanoliter-scale synthesis of arrayed biomaterials and application to human embryonic stem cells. Nat. Biotechnol. 22, 863-866 (2004).

84. Meredith, J. C. et al. Combinatorial characterization of cell interactions with polymer surfaces. J. Biomed. Mater. Res., Part A 66A, 483-490 (2003).

85. Rasi Ghaemi, S. et al. High-Throughput Assessment and Modeling of a Polymer Library Regulating Human Dental Pulp-Derived Stem Cell Behavior. ACS Appl. Mater. Interfaces 10, 38739-38748 (2018).

86. Hay, D. C. et al. Unbiased screening of polymer libraries to define novel substrates for functional hepatocytes with inducible drug metabolism. Stem Cell Res. 6, 92-102 (2011).

87. Mei, Y. et al. Combinatorial development of biomaterials for clonal growth of human pluripotent stem cells. Nat. Mater. 9, 768-778 (2010).

88. Yang, J. et al. Polymer surface functionalities that control human embryoid body cell adhesion revealed by high throughput surface characterization of combinatorial material microarrays. Biomaterials 31, 8827-8838 (2010).

89. Hook, A. L. et al. Combinatorial discovery of polymers resistant to bacterial attachment. Nat. Biotechnol. 30, 868-875 (2012).

90. Sanni, O. et al. Bacterial attachment to polymeric materials correlates with molecular flexibility and hydrophilicity. Adv. Healthc. Mater. 4, 695-701 (2015).

91. Hook, A. L. et al. Discovery of novel materials with broad resistance to bacterial attachment using combinatorial polymer microarrays. Adv. Mater. 25, 2542-2547 (2013).

92. Brocchini, S., James, K., Tangpasuthadol, V. \& Kohn, J. Structure-property correlations in a combinatorial library of degradable biomaterials. J. Biomed. Mater. Res. 42, 66-75 (1998).

93. Engler, A. J., Sen, S., Sweeney, H. L. \& Discher, D. E. Matrix Elasticity Directs Stem Cell Lineage Specification. Cell 126, 677-689 (2006).

94. Gilbert, P. et al. Substrate elasticity regulates skeletal muscle stem cell self-renewal in culture. Science (80-. ). 329, 1078-1081 (2011).

95. Gobaa, S. et al. Artificial niche microarrays for probing single stem cell fate in high throughput. Nat. Methods 8, 949-955 (2011).

96. Ranga, A. et al. 3D niche microarrays for systems-level analyses of cell fate. Nat. Commun. 5, 110 (2014).

97. Usprech, J., Romero, D. A., Amon, C. H. \& Simmons, C. A. Combinatorial screening of 3D biomaterial properties that promote myofibrogenesis for mesenchymal stromal cell-based heart valve tissue engineering. Acta Biomater. 58, 34-43 (2017).

98. Liu, H. et al. Microdevice arrays with strain sensors for 3D mechanical stimulation and monitoring of engineered tissues. Biomaterials 172, 30-40 (2018).

99. Duffy, C., Venturato, A., Callanan, A., Lilienkampf, A. \& Bradley, M. Arrays of 3D doublenetwork hydrogels for the high-throughput discovery of materials with enhanced physical and biological properties. Acta Biomater. 34, 104-112 (2016).

100. Zhang, R., Liberski, A., Sanchez-Martin, R. \& Bradley, M. Microarrays of over 2000 hydrogels Identification of substrates for cellular trapping and thermally triggered release. Biomaterials 30, 6193-6201 (2009).

101. Kumachev, A. et al. High-throughput generation of hydrogel microbeads with varying elasticity for cell encapsulation. Biomaterials 32, 1477-1483 (2011).

102. Iansante, V., Dhawan, A., Masmoudi, F. \& Lee, C. A. A New High Throughput Screening Platform for Cell Encapsulation in Alginate Hydrogel Shows Improved Hepatocyte Functions by Mesenchymal Stromal Cells. Front. Med. 5, 1-11 (2018).

103. Li Jeon, N. et al. Neutrophil chemotaxis in linear and complex gradients of interleukin-8 formed in a microfabricated device. Nat. Biotechnol. 20, 826-830 (2002).

104. Chiu, D. T. et al. Patterned deposition of cells and proteins onto surfaces by using threedimensional microfluidic systems. Proc. Natl. Acad. Sci. 97, 2408-2413 (2000). 
105. Barata, D., Provaggi, E., Van Blitterswijk, C. \& Habibovic, P. Development of a microfluidic platform integrating high-resolution microstructured biomaterials to study cell-material interactions. Lab Chip 17, 4134-4147 (2017).

106. Huh, D. et al. A human disease model of drug toxicity-induced pulmonary edema in a lung-ona-chip microdevice. Sci. Transl. Med. 4, (2012).

107. Barata, D., Van Blitterswijk, C. \& Habibovic, P. High-throughput screening approaches and combinatorial development of biomaterials using microfluidics. Acta Biomater. 34, 1-20 (2016).

108. Mi, S. et al. Microfluidic co-culture system for cancer migratory analysis and anti-metastatic drugs screening. Sci. Rep. 6, 1-11 (2016).

109. Zhang, Y., Zhang, W. \& Qin, L. Mesenchymal-Mode Migration Assay and Antimetastatic Drug Screening via High Throughput Microfluidics Channel Networks. Angew Chem Int Ed Engl 38, 469-485 (2014).

110. Chung, B. G. et al. Human neural stem cell growth and differentiation in a gradient-generating microfluidic device. Lab Chip 5, 401-406 (2005).

111. Kim, J. H., Sim, J. \& Kim, H. J. Neural stem cell differentiation using microfluidic devicegenerated growth factor gradient. Biomol. Ther. 26, 380-388 (2018).

112. Occhetta, P., Visone, R. \& Rasponi, M. High-throughput microfluidic platform for 3D cultures of mesenchymal stem cells. Sci. Rep. 1612, 303-323 (2017).

113. Klein, A. M., Mazutis, L., Akartuna, I., Tallapragada, N. \& Veres, A. Droplet barcoding for single cell transcriptomics applied to embryonic stem cells. Cell 161, 1187-1201 (2016).

114. Caen, O., Lu, H., Nizard, P. \& Taly, V. Microfluidics as a Strategic Player to Decipher SingleCell Omics? Trends Biotechnol. 35, 713-727 (2017).

115. Ben-David, U. et al. Selective elimination of human pluripotent stem cells by an oleate synthesis inhibitor discovered in a high-throughput screen. Cell Stem Cell 12, 167-179 (2013).

116. Borowiak, M. et al. Small Molecules Efficiently Direct Endodermal Differentiation of Mouse and Human Embryonic Stem Cells. Cell Stem Cell 4, 348-358 (2009).

117. Ding, S. et al. Synthetic small molecules that control stem cell fate. PNAS 100, 7632-7637 (2003). 118. Alves, H., Dechering, K., van Blitterswijk, C. \& de Boer, J. High-throughput assay for the identification of compounds regulating osteogenic differentiation of human mesenchymal stromal cells. PLoS One 6, 2-11 (2011).

119. Chen, S. et al. Self-renewal of embryonic stem cells by a small molecule. Proc. Natl. Acad. Sci. 103, 17266-17271 (2006).

120. Le, B. q. et al. High-Throughput Screening Assay for the Identification of Compounds Enhancing Collagenous Extracellular Matrix Production by ATDC5 Cells. Tissue Eng. Part C Methods 21, 726-736 (2015).

121. Doorn, J. et al. A small molecule approach to engineering vascularized tissue. Biomaterials 34, 3053-3063 (2013).

122. Westbroek, P. \& Marin, F. A marriage of bone and nacre. Nature 392, 861-862 (1998).

123. Crubzy, E., Murail, P., Girard, L. \& Bernadou, J.-P. False teeth of the Roman world. Nature 391, 29-29 (1998).

124. Nerlich, A. G., Zink, A., Szeimies, U. \& Hagedorn, H. G. Ancient Egyptian prosthesis of the big toe. Lancet 356, 2176-2179 (2000).

125. Zhao, Y. et al. High-definition micropatterning method for hard, stiff and brittle polymers. Mater. Sci. Eng. C 71, 558-564 (2017).

126. Kurpinski, K., Chu, J., Hashi, C. \& Li, S. Anisotropic mechanosensing by mesenchymal stem cells. Proc. Natl. Acad. Sci. U. S. A. 103, 16095-100 (2006).

127. Berthier, E., Young, E. W. K. \& Beebe, D. Engineers are from PDMS-land, biologists are from polystyrenia. Lab Chip 12, 1224-1237 (2012).

128. Goral, V. N., Hsieh, Y. C., Petzold, O. N., Faris, R. A. \& Yuen, P. K. Hot embossing of plastic microfluidic devices using poly(dimethylsiloxane) molds. J. Micromechanics Microengineering 21, (2011).

129. Peng, L., Deng, Y., Yi, P. \& Lai, X. Micro hot embossing of thermoplastic polymers: A review. J. Micromechanics Microengineering 24, (2014).

130. Santini, A., Gallegos, I. T. \& Felix, C. M. Photoinitiators in dentistry: a review. Prim. Dent. J. 2, 30-33 (2013). 
131. Çiçek, Ç., Çakmakçi, E., Kayaman-Apohan, N., Arslan, M. \& Erdem Kuruca, S. Fabrication of PLGA based tissue engineering scaffolds via photocuring and salt leaching techniques. Int. J. Polym. Mater. Polym. Biomater. 62, 719-725 (2013).

132. Choi, J. R., Yong, K. W., Choi, J. Y. \& Cowie, A. C. Recent Advances in Hydrogels for Biomedical Applications. Biotechniques 66, 40-53 (2019).

133. Vrij, E. J. et al. 3D high throughput screening and profiling of embryoid bodies in thermoformed microwell plates. Lab Chip 16, 734-742 (2016).

134. Mair, D. A., Geiger, E., Pisano, A. P., Fréchet, J. M. J. \& Svec, F. Injection molded microfluidic chips featuring integrated interconnects. Lab Chip 6, 1346-1354 (2006).

135. Truckenmüller, R. et al. Thermoforming of film-based biomedical microdevices. Adv. Mater. 23, 1311-1329 (2011).

136. Tu, C. et al. A Microfluidic Chip for Cell Patterning Utilizing Paired Microwells and Protein Patterns. Micromachines 8, (2017).

137. Kane, R. S., Takayama, S., Ostuni, E., Ingber, D. E. \& Whitesides, G. M. Patterning proteins and cells using soft lithography. Biomaterials 20, 2363-2376 (1999).

138. Duan, X. et al. High-resolution contact printing with chemically patterned flat stamps fabricated by nanoimprint lithography. Adv. Mater. 21, 2798-2802 (2009).

139. Granlund, T., Nyberg, T., Stolz Roman, L., Svensson, M. \& Inganäs, O. Patterning of polymer light-emitting diodes with soft lithography. Adv. Mater. 12, 269-273 (2000).

140. Bennett, R. D. et al. Creating patterned carbon nanotube catalysts through the microcontact printing of block copolymer micellar thin films. Langmuir 22, 8273-8276 (2006).

141. Wang, M., Braun, H. G. \& Meyer, E. Patterning of polymeric/inorganic nanocomposite and nanoparticle layers. Chem. Mater. 14, 4812-4818 (2002).

142. Alom, S. \& Chen, C. S. Microcontact printing: A tool to pattern. Soft Matter 3, 168-177 (2006).

143. Nie, Z. \& Kumacheva, E. Patterning surfaces with functional polymers. Nat. Mater. 7, 277-290 (2008).

144. Kaufmann, T. \& Ravoo, B. J. Stamps, inks and substrates: Polymers in microcontact printing. Polym. Chem. 1, 371-387 (2010).

145. Zhang, R., Liberski, A., Khan, F., Diaz-Mochon, J. J. \& Bradley, M. Inkjet fabrication of hydrogel microarrays using in situ nanolitre-scale polymerisation. Chem. Commun. 1317-1319 (2008). doi:10.1039/b717932d

146. De Gans, B. J., Duineveld, P. C. \& Schubert, U. S. Inkjet printing of polymers: State of the art and future developments. Adv. Mater. 16, 203-213 (2004).

147. Balakirev, M. Y. et al. Photochemical patterning of biological molecules inside a glass capillary. Anal. Chem. 77, 5474-5479 (2005).

148. Kim, M. et al. Addressable micropatterning of multiple proteins and cells by microscope projection photolithography based on a protein friendly photoresist. Langmuir 26, 12112-12118 (2010).

149. R Core Team. R: A language and environment for statistical computing. R Found. Stat. Comput.

150. Reference, P. L. Python Software Foundation.

151. RStudio Team. RStudio: Integrated Development for R. (2015).

152. Carpenter, A. E. et al. CellProfiler: image analysis software for identifying and quantifying cell phenotypes. Genome Biol. 7, R100 (2006).

153. Schindelin, J. et al. Fiji - an Open platform for biological image analysis. Nat. Methods 9, 241 (2009).

154. Mierswa, I. \& Klinkenberg, R. RapidMiner Studio. (2018).

155. Ramakrishnaiah Siddappa, Ruud Licht, Clemens van Blitterswijk, J. de B. Donor Variation and Loss of Multipotency during In Vitro Expansion of Human Mesenchymal Stem Cells for Bone Tissue Engineering. J. Orthop. Res. (2007). doi:10.1002/jor.20402

156. Leek, J. T. et al. Tackling the widespread and critical impact of batch effects in high-throughput data. Nat. Rev. Genet. 11, 733-739 (2010).

157. Leek, J. T. \& Storey, J. D. Capturing heterogeneity in gene expression studies by surrogate variable analysis. PLoS Genet. 3, 1724-1735 (2007).

158. Zijl, S. et al. Micro-scaled topographies direct differentiation of human epidermal stem cells. Acta Biomater. (2018). doi:10.1016/j.actbio.2018.12.003 
159. Bray, M.-A. et al. Cell Painting, a high-content image-based assay for morphological profiling using multiplexed fluorescent dyes. Nat. Protoc. 11, 049817 (2016).

160. McQuin, C. et al. CellProfiler 3.0: Next-generation image processing for biology. PLoS Biol. 16, $1-17$ (2018).

161. Moffat, J. et al. A Lentiviral RNAi Library for Human and Mouse Genes Applied to an Arrayed Viral High-Content Screen. Cell 124, 1283-1298 (2006).

162. Wen, J. H. et al. Interplay of matrix stiffness and protein tethering in stem cell differentiation. Nat. Mater. 13, 979-987 (2014).

163. Diem, K. et al. Image analysis for accurately counting CD4+ and CD8+ T cells in human tissue. J. Virol. Methods 222, 117-121 (2015).

164. Tollemar, V. et al. Quantitative chromogenic immunohistochemical image analysis in cellprofiler software. Cytom. Part A 1-9 (2018). doi:10.1002/cyto.a.23575

165. Caicedo, J. C. et al. Nucleus segmentation across imaging experiments: the 2018 Data Science Bowl. Nat. Methods 16, 1247-1253 (2019).

166. Caicedo, J. C. et al. Evaluation of Deep Learning Strategies for Nucleus Segmentation in Fluorescence Images. Cytom. Part A 95, 952-965 (2019).

167. Sommer, C., Hoefler, R., Samwer, M. \& Gerlich, D. W. A deep learning and novelty detection framework for rapid phenotyping in high-content screening. Mol. Biol. Cell 28, 3428-3436 (2017).

168. Doan, M. et al. Label-Free Leukemia Monitoring by Computer Vision. Cytom. Part A (2020). doi:10.1002/cyto.a.23987

169. Jeyaraj, P. R. \& Samuel Nadar, E. R. Computer-assisted medical image classification for early diagnosis of oral cancer employing deep learning algorithm. J. Cancer Res. Clin. Oncol. 145, 829-837 (2019).

170. Urban, G. et al. Deep Learning Localizes and Identifies Polyps in Real Time With 96\% Accuracy in Screening Colonoscopy. Gastroenterology 155, 1069-1078.e8 (2018).

171. Ronneberger, O., Fisher, P. \& Brox, T. U-Net: Convolutional Networks for Biomedical Image Segmentation. MICCAI 9351, 234-241 (2015).

172. Sadanandan, S. K., Ranefall, P., Guyader, S. Le \& Wählby, C. Automated Training of Deep Convolutional Neural Networks for Cell Segmentation. Sci. Rep. 7, 1-7 (2017).

173. Yang, S. J. et al. Assessing microscope image focus quality with deep learning. BMC Bioinformatics 19, 1-9 (2018).

174. Van Valen, D. A. et al. Deep Learning Automates the Quantitative Analysis of Individual Cells in Live-Cell Imaging Experiments. PLoS Comput. Biol. 12, 1-24 (2016).

175. Moen, E. et al. Deep learning for cellular image analysis. Nat. Methods 16, 1233-1246 (2019).

176. Fourcade, A. \& Khonsari, R. H. Deep learning in medical image analysis: A third eye for doctors. J. Stomatol. Oral Maxillofac. Surg. 120, 279-288 (2019).

177. Vasilevich, A. S., Carlier, A., de Boer, J. \& Singh, S. How Not To Drown in Data: A Guide for Biomaterial Engineers. Trends Biotechnol. 35, 743-755 (2017).

178. Irrechukwu, O. N. et al. Characterization of Engineered Cartilage Constructs Using Multiexponential T 2 Relaxation Analysis and Support Vector Regression. Tissue Eng. Part C Methods 18, 433-443 (2012).

179. Bakker, D. P. et al. Multiple linear regression analysis of bacterial deposition to polyurethane coatings after conditioning film formation in the marine environment. Microbiology 150, 1779-1784 (2004).

180. Sun, Y., Liu, Y., Wang, G. \& Zhang, H. Deep Learning for Plant Identification in Natural Environment. Comput. Intell. Neurosci. 2017, (2017).

181. Fukunaga, K. \& Narendra, P. M. A Branch and Bound Algorithm for Computing k-Nearest Neighbors. IEEE Trans. Comput. C-24, 750-753 (1975).

182. Breiman, L. Random forests. Mach. Learn. 45, 5-32 (2001).

183. Steinwart, I. \& Christmann, A. Support Vector Machines. (Springer, 2008).

184. Lecun, Y., Bengio, Y. \& Hinton, G. Deep learning. Nature 521, 436-444 (2015).

185. Epa, V. C., Yang, J., Mei, Y., Hook, A. L. \& Langer, R. Modelling human embryoid body cell adhesion to a combinatorial library of polymer surfaces. J Mater Chem 22, 20902-20906 (2012).

186. MacQueen, J. Some Methods for Classification and Analysis of Multivaraite Observations. Proc. Fifth Berkeley Symp. Math. Stat. Prob. 1, 281-297 (1967). 
187. Stuart, R. O., Bush, K. T. \& Nigam, S. K. Changes in global gene expression patterns during development and maturation of the rat kidney . Proc. Natl. Acad. Sci. U. S. A. 98, 5649-5654 (2001).

188. Tanaka, T. S. et al. Gene Expression Profiling of Embryo-Derived Stem Cells Reveals Candidate Genes Associated With Pluripotency and Lineage Specificity. Genome Res. 12, 1921-1928 (2014).

189. Winkler, R., Klawonn, F. \& Kruse, R. Fuzzy C-means in high dimensional spaces. Int. J. Fuzzy Syst. Appl. 1, 1-16 (2011).

190. Kumar, G. et al. The determination of stem cell fate by 3D scaffold structures through the control of cell shape. Biomaterials 32, 9188-9196 (2011).

191. Xu, R. \& Wunsch, D. C. Clustering algorithms in biomedical research: A review. IEEE Rev. Biomed. Eng. 3, 120-154 (2010).

192. Ronan, T., Qi, Z. \& Naegle, K. M. Avoiding common pitfalls when clustering biological data. Sci. Signal. 9, (2016).

193. Lu, J. et al. MicroRNA expression profiles classify human cancers. Nature 435, 834-838 (2005).

194. Wold, S., Esbensen, K. I. M. \& Geladi, P. Principal Component Analysis. Chemom. Intell. Lab. Syst. 2, 37-52 (1987).

195. Bach. Le, A. Vasilevich, S. Vermeulen, F. Hulshof, F. Stamatialis, C. van Blitterswijk, J. de B. Micro-Topographies Promote Late Chondrogenic Differentiation Markers in the ATDC5 Cell Line. Tissue Eng. Part A 23, 458-469 (2017).

196. Regier, M. C. et al. The Influence of Biomaterials on Cytokine Production in 3D Cultures. Biomacromolecules 18, 709-718 (2017).

197. Lever, J., Krzywinski, M. \& Altman, N. Principal component analysis. Nat. Methods 14, 641642 (2017).

198. van der Maaten, L. \& Hinton, G. Visualizing Data using t-SNE. J. Mach. Learn. Res. 9, 25792605 (2018).

199. Taskesen, E. \& Reinders, M. J. T. 2D representation of transcriptomes by t-SNE exposes relatedness between human tissues. PLoS One 11, 1-6 (2016).

200. Wagner, D. E. et al. Single-cell mapping of gene expression landscapes and lineage in the zebrafish embryo. Science (80-. ). 360, 981-987 (2018).

201. Luxembourg, S. L., Mize, T. H., McDonnell, L. A. \& Heeren, R. M. A. High-spatial resolution mass spectrometric imaging of peptide and protein distributions on a surface. Anal. Chem. 76, 5339-5344 (2004).

202. Iwasaki, H., Eguchi, S., Ueno, H., Marumo, F. \& Hirata, Y. Mechanical stretch stimulates growth of vascular smooth muscle cells via epidermal growth factor receptor. Am. J. Physiol. Heart Circ. Physiol. 278, H521-9 (2000).

203. Ashe, H. L. Modulation of BMP signalling by integrins. Biochem. Soc. Trans. 44, 1465-1473 (2016).

204. Worthington, J. J., Klementowicz, J. E. \& Travis, M. A. TGFß: A sleeping giant awoken by integrins. Trends Biochem. Sci. 36, 47-54 (2011).

205. Wang, W. et al. Induction of predominant tenogenic phenotype in human dermal fibroblasts via synergistic effect of TGF-beta and elongated cell shape. Am. J. Physiol. Cell Physiol. ajpcell.00300.2015 (2015). doi:10.1152/ajpcell.00300.2015

206. Mateos-Timoneda, M. A., Castano, O., Planell, J. A. \& Engel, E. Effect of structure, topography and chemistry on fibroblast adhesion and morphology. J. Mater. Sci. Mater. Med. 25, 1781-1787 (2014).

207. Rohban, M. H. et al. Systematic morphological profiling of human gene and allele function via cell painting. Elife 6, 1-23 (2017).

208. Bray, M. et al. A dataset of images and morphological profiles of 30000 small-molecule treatments using the Cell Painting assay. Giga Sci. 6, 1-5 (2017).

209. Subramanian, A. et al. A Next Generation Connectivity Map: L1000 Platform and the First 1,000,000 Profiles. Cell 171, 1437-1452.e17 (2017).

210. Vasilevich, A. \& de Boer, J. Robot-scientists will lead tomorrow's biomaterials discovery. Curr. Opin. Biomed. Eng. 6, 74-80 (2018).

211. Pan, F. et al. Topographic effect on human induced pluripotent stem cells differentiation towards neuronal lineage. Biomaterials 34, 8131-8139 (2013). 


\section{Chapter IV}

\section{Identification of Topographical Architectures Supporting the Phenotype of Rat Tenocytes}

Steven Vermeulen ${ }^{1}$, Aliaksei Vasilevich ${ }^{1}$, Dimitrios Tsiapalis ${ }^{2,3}$, Nadia Roumans ${ }^{1}$, Pascal Vroemen ${ }^{1,4}$, Nick R.M. Beijer ${ }^{1}$, Aysegul Dede-Eren ${ }^{1}$, Dimitrios Zeugolis $^{2,3}$, Jan de Boer ${ }^{1}$

${ }^{1}$ Laboratory for Cell Biology-Inspired Tissue Engineering, MERLN Institute, University of Maastricht, Maastricht, The Netherlands.

2 Regenerative, Modular \& Developmental Engineering Laboratory, National University of Ireland Galway, Galway, Ireland

${ }^{3}$ Science Foundation Ireland, Centre for Research in Medical Device, National University of Ireland Galway, Galway, Ireland.

${ }^{4}$ University Eye Clinic Maastricht UMC+, Maastricht University Medical Centre+, Maastricht, The Netherlands

Published in: Acta Biomateralia (2019) 


\section{Abstract}

Tenocytes, the main cell type of the tendon, require mechanical stimuli for their proper function. When the tenocyte environment changes due to tissue damage or by transferring tenocytes from their native environment into cell culture, the signals from the tenocyte niche are lost, leading towards a decline of phenotypic markers. It is known that micro-topographies can influence cell fate by the biomechanical cues they provide. To identify the optimal biomechanical niche in vitro, we seeded tenocytes on the TopoChip, a micro-topographical screening platform, and measured expression of the tendon transcription factor Scleraxis. Through machine learning algorithms, we associated elevated Scleraxis levels with topological design parameters. Fabricating microtopographies with optimal surface characteristics on larger surfaces allowed finding an improved expression of multiple tenogenic markers. However, long-term confluent culture conditions coincided with osteogenic marker expression and the loss of morphological characteristics. In contrast, passaging tenocytes which migrated from the tendon directly on the topography resulted in prolonged elongated morphology and elevated Scleraxis levels. This research provides new insights in how micro-topographies elicit biomechanical cues, and supports the notion that micro-topographical design can be implemented in a new generation of tissue culture platforms supporting the phenotype of tenocytes.

\section{Keywords}

Micro-topography; tenocytes; phenotypic maintenance; machine learning; cell morphology 


\section{Introduction}

The tendon is a connective tissue enabling bone movement through the transmission of mechanical forces originating from the muscle. Injuries in this tissue are common and represent $30 \%$ of musculoskeletal disorders ${ }^{1}$. Low cellular content and hypovascularity in tendons go hand in hand with limited regenerative capability, often leading towards scarred and inferior tissue ${ }^{2}$. Clinical interventions usually do not recover the original tendon architecture, emphasizing the need for new tissue engineering approaches. Recent scientific advances such as the regeneration of a complete epidermis ${ }^{3}$ illustrate the potential of the tissue engineering field for clinical applications. Tissue engineering a functional tendon, however, is hindered by the rapid loss of cellular phenotype in vitro, evidenced by loss of spindle-shaped morphology and tenogenic phenotypical markers ${ }^{4-6}$.

The occurrence of phenotypic drift in cell culture is a generic problem in the tissue engineering field and not only limited to cells of the tenogenic lineage. For example, chondrocytes grown in a monolayer cell culture likewise lose their morphologic characteristics and chondrogenic gene expression ${ }^{7,8}$. Nevertheless, adjusting culture conditions through reducing oxygen tension ${ }^{\text {, }}$ substrate composition ${ }^{10}$ or the addition of growth factors ${ }^{11}$ can positively influence chondrocyte phenotype. Expanding cells in an improved culture environment is not only beneficial for phenotypic characteristics; it allows priming cells for in vivo applications. Examples include the use of cAMP by our lab to improve osteogenic differentiation of mesenchymal stem cells for bone repair ${ }^{12}$ or the self-renewal of muscle stem cells for muscle regeneration through changing the stiffness of the substrate material ${ }^{13}$. These examples illustrate that growing cells in an optimized culture dish can direct cell fate for tissue engineering applications.

Also for tenocytes, external stimuli such as growth factors ${ }^{14,15}$ and small molecules ${ }^{16}$ can positively influence tenocyte characteristics. In vivo, mechanical stimulation enables maintenance of the collagen-rich tendon matrix ${ }^{17}$ and stimulates the expression of tenogenic genes ${ }^{18,19}$. Since tenocytes are mechanosensitive cells, we reasoned that micro-topographical architectures can elicit biomechanical cues and thereby improve their tenogenic characteristics in vitro. In light of this, it is known that geometric sensing can influence cell fate, ranging from inducing proliferation or apoptosis ${ }^{20}$, lineage differentiation 21,22 and maintaining phenotypic characteristics ${ }^{23}$. Of interest, altered geometric environments can activate the MAPK/ERK pathway ${ }^{24}$ or RhoA signaling ${ }^{25}$, both known to effect Early-Growth-Response-Factor 1 (Egr1) 26,27 , a tendon related transcription factor ${ }^{28}$. These findings provide an interesting theoretical framework for studying the effects of micro-topographies on tenocyte phenotype.

Currently, there is no marker uniquely expressed in tenocytes. Nevertheless, since the tendon tissue is composed of an organized assembly of collagen fibers, an optimal expression of matrix proteins is essential for proper tendon maintenance. The most prominent tendon related matrix protein is Collagen-I (Col-I), which compromises the majority of the tendon dry mass ${ }^{29}$. Collagen-III (Col-III) is the next most abundant collagen protein and its expression is associated with wound healing after injury ${ }^{30}$. Other tendon matrix-associated proteins include Tenascin-C 
(Tn-c), which plays a role in collagen fiber orientation ${ }^{31}$ and is known to be upregulated upon mechanical loading ${ }^{32}$. The proteoglycans Decorin (Dec) and Biglycan (Bgn) are essential for proper fiber orientation, the mechanical properties of the tendon ${ }^{33,34}$, and play important roles in repair mechanisms ${ }^{35}$. Also, Tenomodulin (Tnmd), a transmembrane glycoprotein and predominantly expressed in tendons, is essential for proper tenocyte proliferation and collagen fibril maturation ${ }^{36}$. Knock-out models of the transcription factors Scleraxis (Scx), Mohawk Homeobox Protein $(\mathrm{Mkx})$ and Egr-1 coincide with reduced expression of the before mentioned proteins and lead to disturbed tendon development 28,37,38. Both Egr-1 and Mkx can upregulate the expression of Tnmd and Col-I upon mechanical stimuli ${ }^{19}$, with Egr-1 also influencing the expression of $\mathrm{Scx}^{39}$. In tendons, $\mathrm{Scx}$ is upregulated upon physical stimulation ${ }^{40}$ and acts upon the expression of Col-I ${ }^{41}$ and Tnmd ${ }^{42}$. Due to the strong relationship between mechanical stimulation and tenogenic gene expression, it is not surprising that a loss of mechanical stimuli is detrimental to healing after injury, while mechanical stimulation improving clinical outcome 43. Of interest, overloading can cause the expression of chondrogenic and osteogenic markers ${ }^{18}$, potentially leading towards tendinopathy. These research findings illustrate the importance of mechanical stimulation on proper tenocyte function for maintaining a healthy tendon.

To find an optimal in vitro biomechanical niche, we utilized the TopoChip, a high-throughput screening platform with 2176 uniquely designed micro-topographies ${ }^{44}$. Previous screens performed with this platform successfully identified topographies enhancing osteogenic differentiation of mesenchymal stem cells ${ }^{45}$ and chondrogenic marker expression of the ATDC5 cell line ${ }^{46}$. Due to its importance for proper tenocyte function, and known activation upon mechanical stimuli, we chose Scx as screening target. We further utilized Scx promoting microtopographical surfaces for studying the behavior of tenocytes on these culture platforms and their exerted influence on tenogenic and non-tenogenic marker expression. In this study, we illustrate that micro-topographical designs can be implemented in tissue culture platforms providing a biomechanical stimulus which improves the phenotype of in vitro cultured tenocytes. 


\section{Results}

\section{Tenocytes lose morphological characteristics in vitro and SCX levels in confluent culture conditions}

In order to assess the effect of topographical cues on tenocyte markers, we first investigated the behavior of tenocytes on standard, flat polystyrene. When comparing the morphology after passaging, it became clear that their original spindle shape characteristics were lost towards a spread morphology with a denser F-actin fiber architecture, coinciding with a flattened nucleus (Fig. 1a). In order to have sufficient cell numbers for downstream experiments, we expanded these tenocytes and investigated how in vitro culture conditions affect Scleraxis (SCX) levels, a tenogenic related transcription factor. All cells showed a positive SCX signal, which was, as expected, localized in the nucleus. We noticed that SCX levels remained constant when cells were passaged every 3-4 days and fixed 24 hours later (Fig. 1b). We further validated this observation by quantifying SCX nuclear intensity levels and found no differences at these different passages (Fig. 1c). Next, we were interested in evaluating SCX levels of cells grown in prolonged confluent culture conditions. In contrast to cells that were passaged, we observed a lower SCX nuclear signal after 7 culture days. This decline continued and after 21 days SCX nuclear localization was absent (Fig. 1d). Quantification of SCX intensity levels verified this phenomenon (Fig. 1e; $* \mathrm{P}<0.05,{ }^{* *} \mathrm{P}<0.01$ ). These observations confirm that tenocytes rapidly lose their morphological characteristics in vitro and that the culture method (passaging or a confluent monolayer) can have a profound effect on tenogenic marker expression.

a

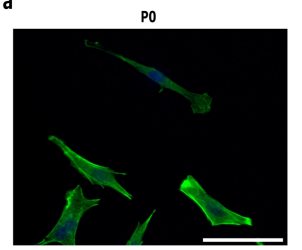

b

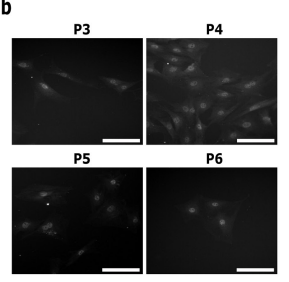

d

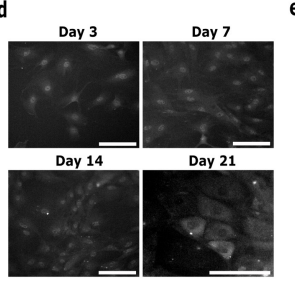

C

e
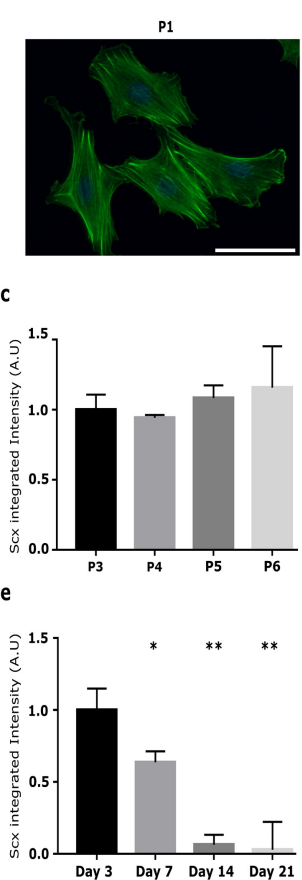

Fig. 1: Tenocytes lose phenotypic characteristics in vitro. a) Representative ICC images of passage 0 and 1 rat tenocytes cultured on a PS flat surface. Tenocytes obtained a spread, wing-like cell morphology and flattened nucleus after passaging. Tenocytes were stained with Phalloidin-568 to visualize F-actin filaments (green) and counterstained with Hoechst to visualize the nucleus (blue). b) Tenocytes stained positive for SCX, and serial passaging resulted in constant SCX levels. c) Quantitative analysis of SCX intensities confirmed constant SCX levels when cells are passaged. Bar chart represents the median of integrated SCX intensities measured in the nucleus. Error bars represent $95 \%$ confidence interval. d) In a confluent culture, a steady decline in SCX levels was observed. At 21 days, SCX nuclear localization was undetectable. For visibility purposes, the contrast was enhanced for the 21-day time point. e) Quantitative analysis confirmed a steady decline in SCX intensity levels for confluent culture conditions. Bar chart represents the median of integrated SCX intensities measured in the nucleus. Error bars represent 95\% confidence intervals $(* \mathrm{P}<0.05$; $* * \mathrm{P}<0.01)$. Scale bars represents $100 \mu \mathrm{m}$. 


\section{TopoChip screen identifies micro-topographies elevating SCX levels in tenocytes}

Since in vitro culture conditions induce changes in tenocyte phenotype, we investigated if microtopographical architectures can positively influence SCX levels and morphological characteristics of these dedifferentiated tenocytes. For this, we produced TopoChips of polystyrene (PS), the standard material used in cell culture. The quality of the spatial dimensions of the topographical features $(10 \mu \mathrm{m}$ height $)$ and the walls $(30 \mu \mathrm{m}$ height) separating the individual units $(290 \times 290$ $\mu \mathrm{m})$ each containing a unique micro-topographical design was assessed by profilometric imaging (Fig. 2a). The height profile of the TopoChip can be found in Supplementary Figure 1. Next, tenocytes were seeded on 6 TopoChips and cultured for 48 hours after which F-actin, DNA, and SCX were fluorescently labeled. This enabled high-content imaging whereby 24 flat surfaces and 12 replicas of each unique micro-topography were analyzed. In total, 29,534 cellular objects were identified. Nuclear SCX intensity values of each individual cell were measured and used to calculate the SCX median intensity per TopoUnit, thus allowing evaluation of the performance of each individual micro-topographical design on SCX expression. The flat surface belonged to the 100 surfaces inducing lowest SCX expression, which was approximately 2-fold lower compared to the 100 surfaces inducing highest SCX expression (Fig. 2b; * P<0.001). No significant difference was measured between the flat surfaces and the lowest top 100 scoring micro-topographies. However, a significant difference was found on 1023/2176 surfaces which induced higher SCX levels compared to flat $(\mathrm{P}<0.05)$. Fig. 2c further illustrates that the majority of micro-topographies induce higher SCX levels compared to the flat surface. Visual inspection of the morphological characteristics of cells cultured on the topographies revealed cell morphology heterogeneity between topographies and their replicas (Fig. 2d). Tenocytes either spread out on top of the micro-topographies, similar to when grown on a flat surface, while others obtained elongated characteristics. These observations demonstrate that microtopographies can induce higher SCX levels in tenocytes, yet accompanied by a heterogeneous response in cell morphology. 
a

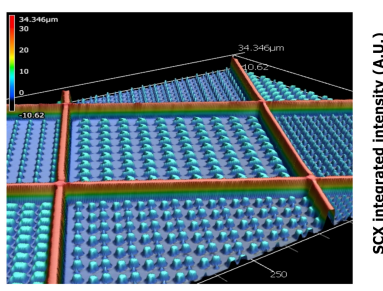

C

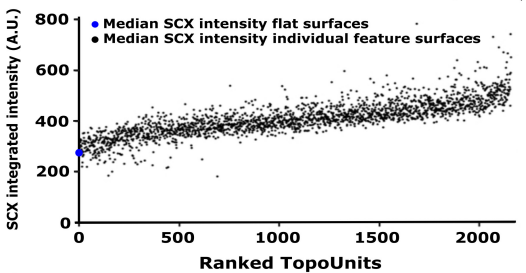

d

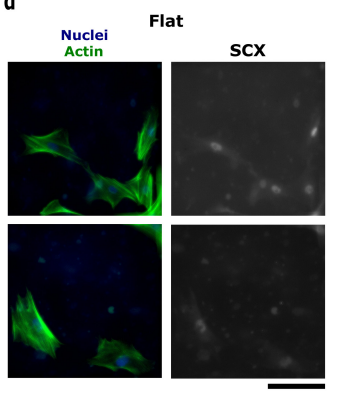

High Hit

Nuclei
Actin
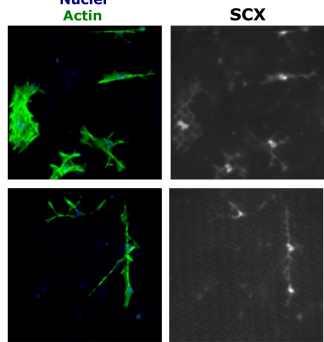

\section{b}

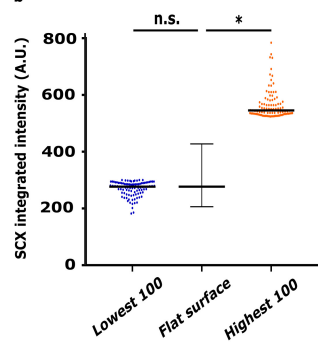

Fig. 2: TopoChip screen identified microtopographies inducing elevated SCX levels in tenocytes after $\mathbf{4 8}$ hours of culturing. a)

Profilometric quality control before cell seeding confirms structural integrity of TopoChip features. b) Dot chart represents median SCX intensity levels of individual TopoUnits of either the top 100 lowest or highest SCX expressing units $(* \mathrm{P}<0.001)$. Median is represented by a horizontal line. Errorbars represent interquartile range. c) Dot chart representing median SCX integrated expression levels of tenocytes cultured on the TopoChip of each individual TopoUnit, ranked according to statistical significance. 1023/2176 surfaces were considered to be statistical significant $(\mathrm{P}<0.05)$. The blue dot represents Scleraxis median integrated intensity of the flat surfaces on the TopoChip. d) Representative ICC images of tenocytes cultured on flat, low and high hit topographies. Two replicas per topography are shown. Cells were stained for F-Actin through Phalloidin-568 and counterstained with Hoechst for nuclear visualization. Alexafluor-647 was used to visualize SCX after primary antibody binding. Scale bar represents $100 \mu \mathrm{m}$. 


\section{SCX expression is associated with topographical and morphological shape descriptors}

After observing that the majority of micro-topographies induce higher SCX levels, we were interested in identifying the relationship between surface design, cell morphological parameters, and SCX expression. The surface architectures can be described through multiple parameters, including feature size, pattern area, the number of primitives used and wavenumbers (WN). A complete description of these parameters can be found in Supplementary Data File 1. In order to characterize the relationship between these surface parameters and SCX expression, we employed a Random Forest algorithm with binary classification. For creating the model we used

a

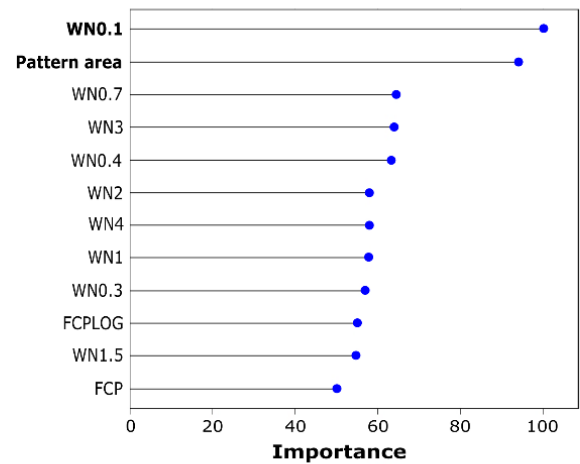

C

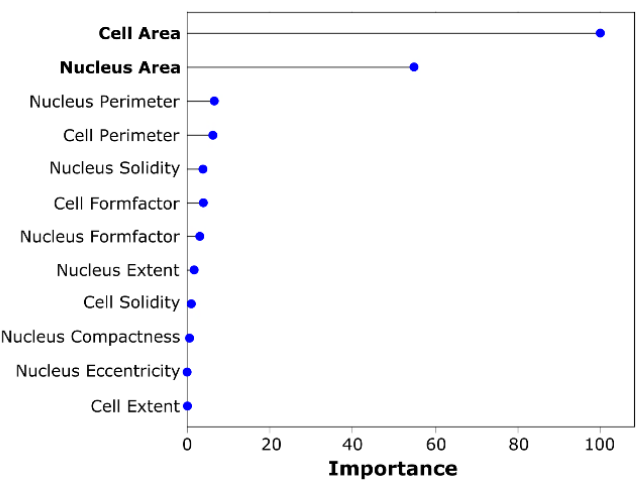

b

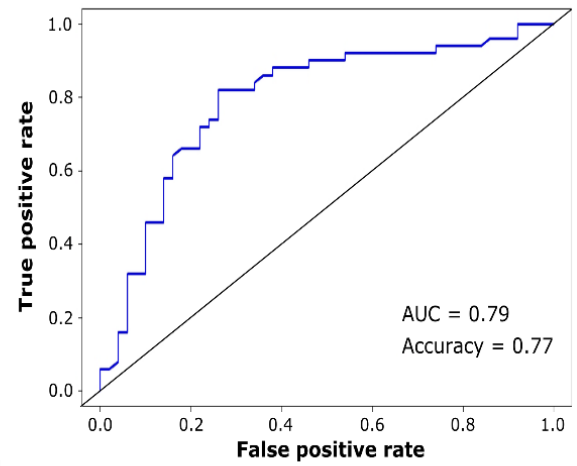

d

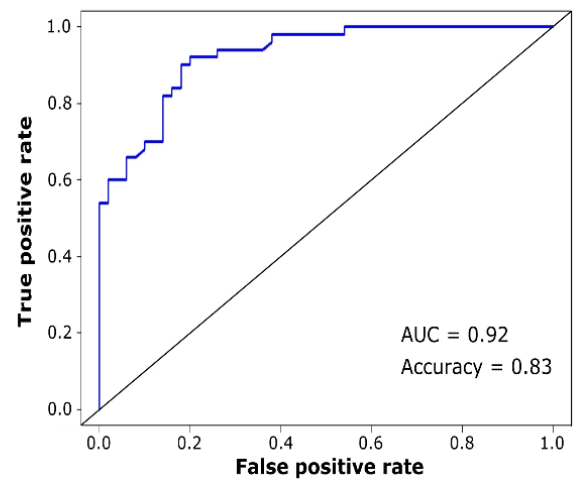

Fig. 3: Identification of topographical and morphological feature parameters associated with increased Scleraxis expression. a) Bar diagram illustrating the importance of topographical feature design parameters as predictive factors for SCX expression through random forest algorithms. Pattern area and wavenumber 0.1 are the most prominent features to distinguish low and high SCX inducing Topo units. b) ROC curve showing the prediction performance of the random forest classification algorithm. The AUC is the area under the curve which indicates the predictive power of the model. c) Bar diagram representing the importance of morphological parameters as predictive factors for SCX expression through random forest algorithms. The cell area is the most prominent feature next to the nuclear area to distinguish low from high SCX expressing Topo units. d) ROC curve showing the prediction performance of the random forest classification algorithm. The AUC is the area under the curve, which indicates the predictive power of the model. 
$75 \%$ of the 200 lowest and highest SCX scoring Topo units. The accuracy of the model was assessed on the remaining 25\%. Models were trained with 10-fold cross-validation. Here, we found that SCX expression can be predicted most prominently based on pattern area and wavenumber 0.1 (Fig. 3a). Applying this model can predict with high accuracy (77\% accuracy; $79 \%$ area under the curve (AUC)) SCX intensity levels based on surface parameters (Fig. 3b). An AUC $>0.6$ is typically regarded as signifying a positive signal.

a

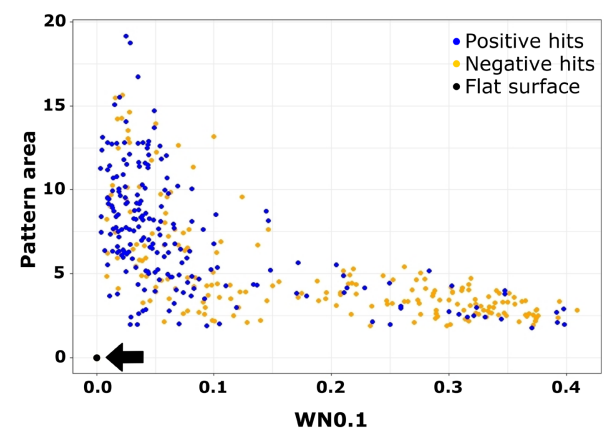

C



d

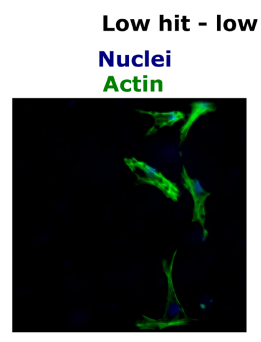

b
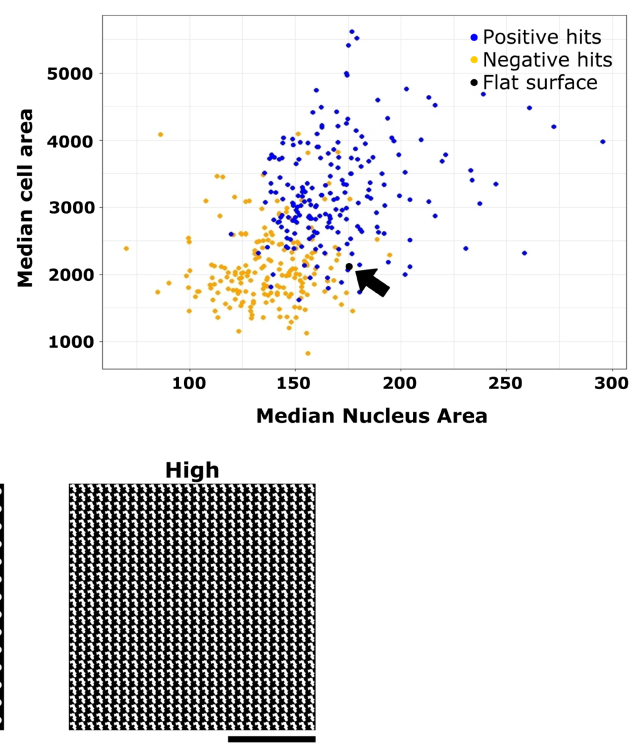

Top hit - high pattern area

Nuclei

Actin

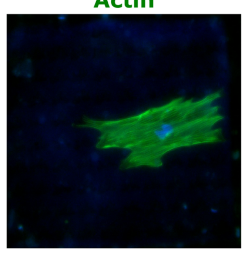

$\operatorname{SCX}$

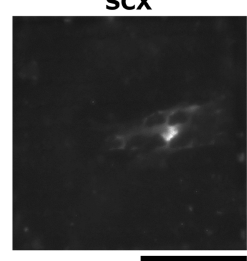

Fig. 4: High pattern area and low WN0.1 promote Scleraxis expression. a) Scatter plot showing the distribution of pattern area and wavenumber 0.1 for topographies with the highest (blue; top 200 hits) and lowest (orange; bottom 200 hits) SCX inducing micro-topographies. b) Scatter plot showing the distribution of cellular and nuclear area for topographies with the lowest (orange; bottom 200 hits) and highest (blue; top 200 hits) SCX levels. c) Illustration of in silico topographical designs with low, intermediate and high pattern area. Scale bar represents $100 \mu \mathrm{m}$. d) Tenocytes grown on higher pattern areas are associated with more cell spreading and elevated SCX levels. Cells were stained for F-actin through Phalloidin-568 and counterstained with Hoechst for nuclear visualization. Alexafluor-647 was used to visualize SCX after primary antibody binding. Scale bar represents $100 \mu \mathrm{m}$. 
The same models were applied to correlate cell morphological features to SCX expression. Through CellProfiler ${ }^{47}$, we extracted multiple quantitative parameters describing morphologic characteristics. These include cell and nuclear area, yet also compactness and solidity, which can describe cell elongation or branching. An overview of morphological feature parameters and their explanation can be found in Supplementary Data File 2. Applying the same random forest algorithm identified cell and nuclear area as the most prominent morphological features for distinguishing cells grown on the lowest and highest SCX inducing Topo units (Fig. 3c). Here, the model can predict with very high accuracy ( $92 \%$ accuracy; $83 \%$ AUC) SCX intensity levels based on morphological parameters (Fig. 3d). Of interest, parameters that define elongation or branching were unimportant in determining SCX levels, which is in sharp contrast with previous screens performed with mesenchymal stem cells, which exhibited a more diverse morphologic response when grown on the micro-topographies ${ }^{45}$. This indicates that the morphologic response that micro-topographies elicit on cells can vary strongly depending on the cell type used.

Fig. 4a illustrates how these models can successfully separate the lowest 200 and highest 200 SCX inducing micro-topographical designs based on WN0.1 and pattern area. A low WN0.1 and an intermediate to high pattern area favor elevated SCX expression. Low values of this particular wavenumber are generally associated with higher pattern areas in our micro-topographical design space (Supplementary Figure 2). Fig. 4b illustrates the differences in low, intermediate and high micro-topographical pattern areas. Fig. $4 \mathbf{c}$ highlights the separation of low and high SCX inducing units through the cell and nuclear area. In general, we found that cells grown on microtopographies with a lower pattern area exhibited a less spread out morphology (Fig. 4d). Cells belonging to the highest SCX hits are characterized by a larger cell spreading compared to flat due to a more profound micro-topographical pattern area.

\section{Large surface fabrication allows in-depth investigation of tenocyte behavior on micro-topographies}

To further investigate the influence topographies exert on tenocytes, we fabricated equivalent micro-topographies from the TopoChip in larger surface areas $(\varnothing 1 \mathrm{~cm})$, while maintaining the original feature dimensions. The selected surfaces induced significant higher SCX levels compared to flat when tenocytes were cultured on the TopoChip platform (Fig. 5a; * P<0.001). The enlarged surface area allows the use of other experimental techniques besides immunocytochemistry (ICC). Furthermore, feature design parameters of these surfaces were in the same range as what the random forest algorithms considered to be important for inducing elevated SCX levels (intermediate pattern area and low WN0.1) (Fig. 5b). Based on these considerations; we selected surfaces designated PS-0264, PS-0304, and PS-1538. Fig. 5c depicts the in silico design of surface architecture PS-0304. After fabrication of these surfaces, we applied a quality control with the profilometer to verify structural integrity. In contrast to the TopoChip platform, these constructs do not contain walls of $30 \mu \mathrm{m}$ (Fig. 5d). A detailed height profile of micro-topography PS-0304 is found in Supplementary Figure 3. Representative ICC images 
of tenocytes cultured on surfaces PS-0264, PS-0304 and PS-1538 on the TopoChip platform with nuclear, F-Actin and SCX staining can be found in Supplementary Figure 4.

During the analysis of the TopoChip screen, we noticed cell morphology heterogeneity of cells grown on the replicas of the individual micro-topographical designs. Despite the strong association between increased cell spreading and SCX levels, we did observe that SCX positive cells with an elongated morphology contributed to the observed cell morphology heterogeneity. Therefore we wanted to investigate if cell shape is dynamic in nature on the micro-topographies by monitoring tenocyte behavior for 16 hours. Cells were allowed to adapt and grow for 24 hours on the PS-0304 surface prior to the start of the live cell imaging. We indeed found that cells had diverse morphologic characteristics at the start of the monitoring. Cells had obtained either a spindle shape morphology and were localized between the micro-topographies, or were spreading out and engulfing the structures, with the latter being similar as the morphologies associated with highest SCX levels (Fig. 5e). We observed that spindle shape cells were able to migrate in between the structures, while the spread cells seemed to be immobilized. Cells with both shapes were able to proliferate. Of interest, we noticed that the elongated cells during

a

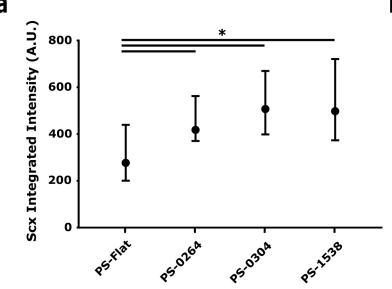

e

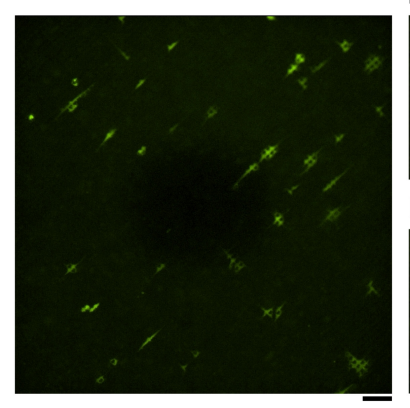

b



c

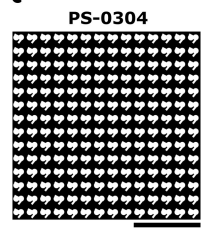

g

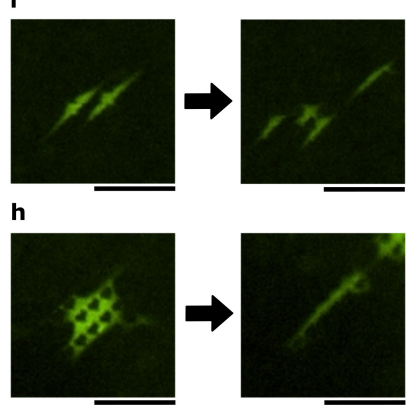

d
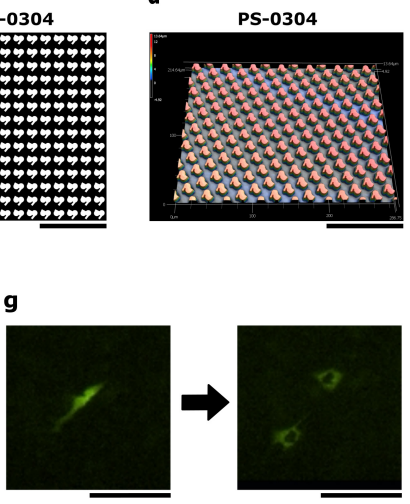

Fig. 5: Micro-topographies fabricated in large surface dimensions allow in-depth investigation of micro-topographical influence on tenocyte behavior. a) Performance of the selected surfaces on the TopoChip platform. Each of the selected surfaces induced significant higher SCX levels compared to flat $(\mathrm{P}<0.001)$. The black dot represents the median, error bars represent interquartile range. b) WN0.1 and pattern area design parameters of the selected surfaces are in comparable ranges as feature design parameters associated with elevated SCX levels. c) Example of an in silico pattern design used for downstream experiments. Depicted is micro-topography PS-0304. Scale bar represents $100 \mu \mathrm{m}$. d) Profilometric quality control of PS-0304 confirms structural integrity before cell seeding. e) After $24 \mathrm{~h}$, tenocytes exhibited either a flattened or elongated morphology on the PS-0304 surface. Scalebar represents $200 \mu \mathrm{m}$. f) During proliferation, tenocytes either kept their elongated morphology or g) lost this characteristic and spread across the micro-topographies immobilizing their migration. $\mathbf{h}$ ) The spread and immobilized tenocytes were able to reobtain elongated characteristics during the 16 hours of culture time. 
proliferation were able to either keep their cell shape (Fig. 5f) or become spread and immobilized upon the topography (Fig. 5g). However, of interest, these immobilized and flattened cells were able to reobtain an elongated morphology during the 16 hour culture period (Fig. 5h). The dynamic behavior of tenocytes on the PS-0304 surface can be studied in further detail in Movie file 1. These observations demonstrate that micro-topographies elicit dynamic cell morphological remodeling on tenocytes.

\section{Micro-topographies increase tenogenic marker expression yet coincides with the expression of chondrogenic and osteogenic markers at later culture times}

To further assess if micro-topographies elicit a beneficial effect on tenocytes or induce dedifferentiation, we performed gene expression analysis on tenogenic and chondrogenic/osteogenic transcription factors, downstream target genes, and tendon matrixrelated proteins, both after 48 hours (Fig. 6a) and 7 days (Fig. 6b). Through immunocytochemistry, we observed an increase of SCX on these large are surfaces after 48 hours, excluding the possibility that the walls of the TopoChip, or growth factors secreted by neighboring Topo units, influenced SCX levels (data not shown). Through qPCR, we further validated the observation that micro-topographies can upregulate $S c x$ gene expression after 48 hours. Of interest, this effect persisted after 7 days with even a 2 -fold increase in $S c x$ transcription levels present compared to the 48 hour culture time. Likewise, we found mild but significantly higher $M k x$ levels for surfaces PS-0264 and PS-1538 compared to flat $(* \mathrm{P}<0.05)$ after 48 hours. However, also here the observed Mkx levels showed a higher elevation for all surfaces after 7 days. Col-I, a downstream target of the before mentioned transcription factors, followed a similar trend as the Mkx gene expression pattern on these surfaces. For the downstream target Tnmd, increased levels were observed both after 48 hours and 7 days. Of interest, no upregulation was found for the transcription factor Egr-1 at the time points investigated.

Sox9, a chondrogenic transcription factor also expressed by tenocytes at the junction between tendon and bone, likewise followed a similar gene expression pattern as Mkx and Col-I for which elevated levels were found on PS-0264 and PS-1538 after 48 hours. Also here, Sox 9 transcription levels were markedly higher after 7 days on all surfaces. For the osteogenic associated transcription factor Runx2, elevated levels were observed after 7 days, highlighting that also osteogenic transcription is activated at this time point.

Of the matrix related proteins, Tn-c showed increased levels both after 48 hours and 7 days with elevated levels of Col-III observable after 7 days. Of interest, Bgn and Dec were not elevated, with even a decrease of Bgn detected at 48 hours for surfaces PS-0264 and PS-0304, which returned to similar levels against flat after 7 days. The expression levels of Aggrecan (Acan), a protein present in both tendons and cartilage and regulated by $S_{0 \times 9}$, was markedly higher after 7 days. Furthermore, also Col-II, regulated by Sox9, and $A \not p$ regulated by Runx2, displayed increased expression levels after 7 days. An overview of the expression profiles of these genes can be found in Supplementary Figure 5. 
Since we did not observe differences in the expression levels of the transcription factor Egr-1 at 48 hours and 7 days, we wondered if we could detect differences in expression levels at earlier time points. For this, we applied immunocytochemistry and detected an increased presence of EGR-1 2 hours after cell seeding on the surfaces (Fig. 6c). Of interest, we observed that at this early time point, all cells exhibit spreading across the micro-topographies. This demonstrates that the morphologic remodeling of tenocytes on micro-topographies is already active at the initial cell-substrate contacts.

a


C

\section{2 hours}
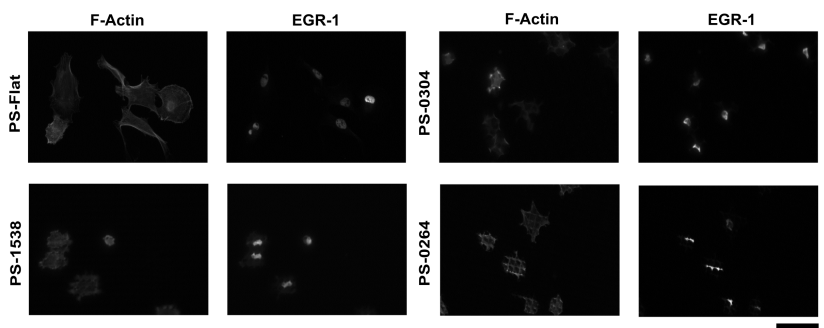

Fig. 6: Gene expression profile of tenocytes grown on the micro-topographical surfaces PS-0264, PS-0304, and PS-1538. a) Bar chart representation of tenogenic, chondrogenic and osteogenic expression levels of tenocytes grown on micro-topographies after 48 hours. Increased levels for the majority of tenogenic markers are observed $(* \mathrm{P}<0.05)$. b) Tenocytes cultured on micro-topographies after 7 days exhibit increased tenogenic marker expression compared to flat, yet coinciding with increased chondrogenic and osteogenic marker expression $(* \mathrm{P}<0.05)$. c) Immunocytochemistry reveals that the mechanosensitive transcription factor EGR-1 is activated early upon seeding on micro-topographies. Cells were stained for F-actin through phalloidin-568. Alexafluor-647 was used to visualize EGR-1 after primary antibody incubation. Scale bar represents $100 \mu \mathrm{m}$. 
The qPCR data confirms that topographies have a positive effect on the gene expression levels of tendon-related markers. However, after 7 days, the upregulation of tenogenic markers coincides with increased levels of chondrogenic and osteogenic markers, indicating that the positive effect of micro-topographies needs further guidance by other environmental stimuli.

\section{Evaluation of serial passaging and confluent culture conditions on tenocytes cultured directly on the PS-0304 platform}

Previous experiments indicate that micro-topographies can have a beneficial effect on tenogenic marker expression, yet with heterogeneous morphologic characteristics and the expression of chondrogenic/osteogenic markers at later time-points. However, until now, we utilized tenocytes which exhibited dedifferentiated morphologic characteristics before seeding on the micro-topographies. For tissue engineering applications, it would be most interesting to evaluate the performance of micro-topographies on tenocytes isolated directly from a tendon tissue. Therefore, we selected the PS-0304 surface, which we manufactured in a $50 \mathrm{~mm}$ diameter dimension. Achilles tendon tissue was isolated from rats and placed on flat and the PS-0304 pattern which allowed the migration of tenocytes directly from a tendon tissue. After 5 days, we observed that cells started migrating on the surface (Fig. 7a-b). Two days later, cells were either fixed for ICC (Passage 0; P0) or passaged. These passaged cells (P1) were either grown to confluency or passaged further every 3 days either on the PS-0304 surface or flat, after which SCX levels were evaluated by ICC after 48 hours. We found only a modest and non-significant increase in SCX expression of the P0 tenocytes grown on the PS-0304 surface compared to flat. However, once cells were reseeded on the topography, we noticed improved SCX levels, and this effect was maintained upon multiple passaging steps (Fig. 7c; $* \mathrm{P}<0.001)$. Furthermore, we observed that cells homogenously retained a small and elongated nuclear and cell morphology (Fig. 7d-e), which is in contrast with the dedifferentiated cells used previously which showed a heterogeneous cell morphological response with a strong spreading across the microtopographies. Quantification of cell spreading revealed that cells grown on topographies had a similar spreading as P0 tenocytes across multiple passages, with confluent cells on PS-0304 losing their spindle-shaped morphology and re-obtaining characteristics similar as cells cultured on flat (Supplementary Figure 5a; $* \mathrm{P}<0.05$; ** P <0.001). Of interest, P0 tenocytes grown on PS0304 had even smaller spreading compared to cells grown on flat. Nuclear dimensions of tenocytes cultured on PS-0304 remained similar as P0 tenocytes and was markedly different compared to tenocytes grown on the flat surface (Supplementary Figure $5 \mathbf{b}$; $* *$ P $<0.001$ ). For tenocytes that were allowed to grow confluent, SCX levels remained elevated compared to flat after 7 days, a similar observation as seen with the qPCR experiments. However, after 14 days SCX levels dropped and micro-topographies were unable to counteract SCX loss as seen with confluent cells grown on the flat surface (Fig. 7f). The loss of spindle-shaped characteristics of tenocytes allowed to grow confluent on PS-0304 demonstrates that microtopographies are unable to counteract dedifferentiation and the loss of SCX levels in these conditions. Nevertheless, it seems that passaging cells on the topographies repeatedly leads to increased SCX expression and maintenance of elongated morphological characteristics, which is 
best applied with tenocytes isolated directly from the tendon tissue and which are not preconditioned in classic culture conditions.

a

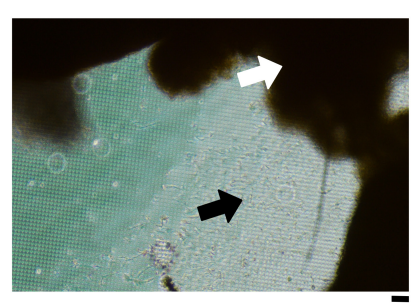

b

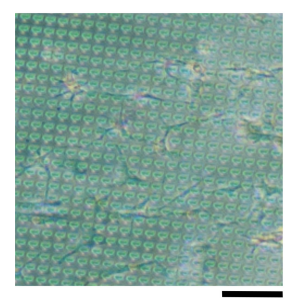

e

d
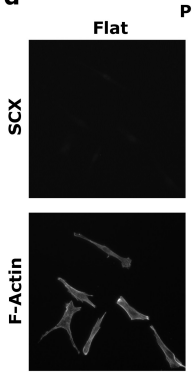

PO PS-0304
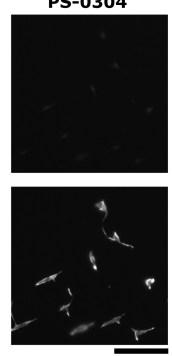


P3

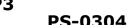

C

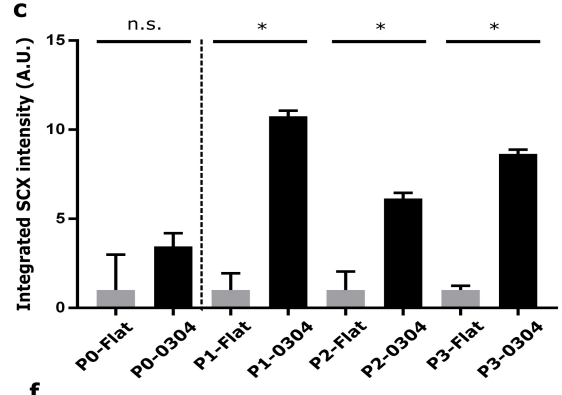

$\mathbf{f}$
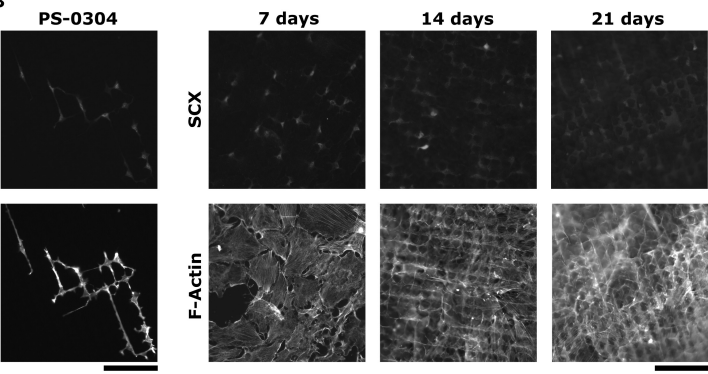

Fig. 7: Evaluation of serial passaging and confluent culture conditions on tenocytes cultured directly on the PS-0304 platform. a) Bright field image of tenocytes migrating out of the tendon tissue on PS-0304. The white arrow directs towards the tendon tissue. The black arrow guides towards tenocytes migrating on PS-0304. Scale bar represents $100 \mu \mathrm{m}$. b) Close-up of P0 tenocytes migrating from the tendon tissue on PS-0304. Scale bar represents $100 \mu \mathrm{m}$. c) ICC quantification of nuclear SCX in tenocytes after serial passaging reveals elevated protein levels. P0 tenocytes grown on the topography show only a slight non-significant elevation. Bar chart represents the median of integrated Scx intensities measured in the nucleus. Error bars represent 95\% confidence interval $(* \mathrm{P}<0.01)$. d) Representative ICC image of P0 tenocytes grown on flat or PS-0304. e) Representative ICC images of P3 tenocytes reveals that cells grown on PS-0304 retain an elongated morphology. f) A drop in SCX intensity levels is observed for tenocytes cultured in confluent conditions on PS-0304 after 14 days. Tenocytes furthermore lose their morphological characteristics. Cells were stained with phalloidin-568 to visualize F-actin filaments. Alexafluor-647 was used to visualize SCX.

\section{Discussion}

This study is the first to assess the performance of substrate structures in micro-topographical dimensions on tenocyte phenotype. Previously, other research groups have investigated the influence of alternative surface structures on tenocyte phenotype by utilizing groove structures in nano- and micrometer dimensions ${ }^{48-50}$. When comparing these studies with our research results, some interesting similarities are observed. In the study of English et al., grooves in micrometer dimensions led to elongated morphological characteristics in tenocytes and also gave rise to an upregulation of Scx and other tenogenic markers ${ }^{48}$. Yet also here, the increased expression of tenogenic markers coincided with the upregulation of chondrogenic and osteogenic markers at later time points (10 days). In another study, micro-grooves in larger 
dimensions $(50-250 \mu \mathrm{m})$ led to increased elongation of tenocytes with an improvement in Col-I and Col-III levels ${ }^{49}$. Of interest, in the study of Zhu et al., culturing tenocytes on grooves in the $\mu \mathrm{m}$ range increased the expression of both Tnmd and Col-I across multiple passages ${ }^{50}$, an approach similar as in this study where we found a repeated upregulation of SCX, upstream of Tnmd and Col-I ${ }^{41,42}$. These research findings support the notion that substrate structures can positively modulate tenogenic gene expression, favoring a culture system where cells are passaged upon these substrates.

The previous statement is further highlighted by the observation that tenocytes reaching confluency on the micro-topographies adapt a spread shape similar as when cultured on flat. This confluent condition is further associated with elevated osteogenic and chondrogenic markers and eventually a decline in SCX levels. Of interest are the elevated levels of EGR-1 at the initial moments of cell-substrate contact, which might be linked with the upregulation of SCX. Although it is beyond the scope of this study to investigate the pathways involved in this phenomenon, a plausible explanation might be RhoA dynamics, previously shown to play a role in increasing the expression of Tnmd and Col-I on microgrooves 50, and tenogenic differentiation of MSCs ${ }^{51,52}$. The influence of RhoA on Egr-1 might be due to the pathways' involvement in ERK signaling ${ }^{53}$. In light of this, we did observe active cytoskeleton remodeling towards a smaller and elongated morphology when tenocytes are cultured on topographies. This could explain why machine learning algorithms indicate an association between elevated SCX expression, a spread morphology and an intermediate to high pattern area, since this will require more cytoskeletal reorganization to adapt to the environment, which we show to be an active process on micro-topographies.

Surprisingly, we found only a slight increase in SCX levels of P0 tenocytes migrating on microtopographies, even with elongated morphological characteristics. However, these tenocytes did not receive any mechanical loading during the first week they were in culture, further emphasizing the observation that micro-topographies initiate a biomechanical stimulus upon reseeding. The resulting beneficial effect on SCX expression was observed on dedifferentiated tenocytes, yet was more pronounced with P0 tenocytes, which also retained their elongated morphologic characteristics upon multiple passages. This could imply that the standard in vitro culturing of tenocytes on flat elicited a mechanical memory. We did observe a higher presence of F-Actin fibers on these dedifferentiated tenocytes already after one passage, which is associated with increased cell stiffness ${ }^{54}$ and might result in a decreased ability to adapt to the micro-topographical environment.

In vivo, Egr-1 expression is elevated shortly upon mechanical stimulation ${ }^{55}$, a similar observation when tenocytes are seeded on micro-topographies. This provokes an interesting hypothesis that micro-topographies can in vitro provide biomechanical stimulation of tenocytes through the same signaling mechanisms as when tenocytes are in vivo subjected to mechanical loading. Nevertheless, when considering the use of micro-topographies in cell culture platforms, the multifactorial nature of the culture system should be taken into account. Although Egr-1 regulates tenogenic signaling upon mechanical stimulation ${ }^{39}$, it is not expressed solely in cells of 
the tenogenic lineage, yet is involved in chondro- and osteogenesis as well 56,57. This might explain the observed early elevation of Sox 9 , a chondrogenic transcription factor ${ }^{58}$. Since tenocyte gene expression is dependent on the location of the tenocytes in the tendon ${ }^{59}$, with even a SCX and SOX9 positive subpopulation existing ${ }^{60}$, it is difficult to assess if the observed gene signature is similar as when tenocytes are in vivo subjected with mechanical stimulation. Nevertheless, since other tenocyte subtypes do not readily express chondrogenic or osteogenic markers, we believe that the biomechanical stimulus elicited by the micro-topographies needs additional guidance from the culture environment. Examples for improving in vitro culture conditions include changing the polystyrene material towards a collagen substrate or altering the growth factor composition of the medium. We conclude by stating that micro-topographical architectures can be applied in tissue culture platforms for providing tenocytes with biomechanical stimulation otherwise absent in classical culture platforms.

\section{Conclusion}

This study illustrates that micro-topographies can positively modulate the expression of tenogenic associated markers. Furthermore, we demonstrated that this is largely dependent on the cell culture context, whereby culturing cells during multiple passages is more advantageous compared to confluent culture conditions. This work supports the concept that microtopographies can provide a xenofree biomechanical niche for supporting the phenotype of tenocytes.

\section{Materials and methods}

\section{TopoChip and large area surface fabrication}

A detailed description of the surface fabrication procedures can be found elsewhere ${ }^{61}$. In short, the in silico design of the TopoChip is generated using an algorithm that combines triangles, circles, and rectangles to generate complex shapes. The combination of these shapes in varying orientations, numbers and sizes leads towards the generation of 154.320 .600 unique topographies. For the TopoChip design, 2176 topographies were randomly selected from this pool and placed in areas of 290x290 $\mu \mathrm{m}$, separated by walls of $30 \mu \mathrm{m}$. All topographies have a height profile of $10 \mu \mathrm{m}$. The inverse pattern of the topographies was etched from a silicon wafer, by directional reactive ion etching (DRIE), generating a silicon master mould for hot embossing. To facilitate the demoulding procedure, the master was coated with a layer of perfluorodecyltrichlorosilane (FDTS, Sigma-Aldrich). Polydimethylsiloxane (PDMS; Down Corning) was used to generate a positive mould. The PDMS mould was subsequently used to create a second negative mould in OrmoStamp ${ }^{\circledR}$ hybrid polymer (micro resist technology Gmbh), which serves as the mould for hot embossing the PS films (Goodfellow). The conditions for the hot embossing were $140^{\circ} \mathrm{C}$ for 5 min at a pressure of 10 Bar, with a demoulding temperature of $90^{\circ} \mathrm{C}$. For micro-topographies imprinted in larger surface areas $(\varnothing 1-5 \mathrm{~cm})$ the same procedures were performed. For these surfaces, micro-topographies with the same feature 
parameters as the TopoChip are etched across larger areas and without $30 \mu \mathrm{m}$ walls. Before cell culture, the PS topographies were treated with oxygen plasma to improve cell adhesion. The plasma treatment was performed for $30 \mathrm{~s}$ at $75 \mathrm{~m}$ Tor, $50 \mathrm{sccm} \mathrm{O}_{2}$, and $50 \mathrm{~W}$. Quality of the fabricated imprints was assessed using a Keyence VK-H1XM-131 profilometer.

The topographical numbering utilized in this manuscript is based on our TopoUnit database, a repository containing the design parameters of each individual micro-topography. For example, T2-PS-0304 refers to the second generation of TopoChip design (T2), of which the microtopographies are imprinted on polystyrene (PS). The first two digits represent the row number starting from the top of a TopoChip, while the second two digits represent the column number. Throughout the manuscript, we will apply the abbreviation PS-0304.

\section{Tenocyte isolation and cell culture}

A protocol describing the isolation of rat tenocytes is described elsewhere ${ }^{48}$. Rat tendon tissues were harvested post-mortem from rats enrolled in studies approved by the institutional Ethics Committee of NUI Galway. The isolated tenocytes were placed in culture flasks in DMEM low glucose (Merck) supplemented with 10\% (v/v) FCS (Merck) and Penicillin/Streptomycin (100 $\mathrm{U} / \mathrm{ml}$; ThermoFisher). Cells were grown at $37{ }^{\circ} \mathrm{C}$ in a humid atmosphere with $5 \% \mathrm{CO}_{2}$. Media was changed every 2-3 days. Cells were seeded at $5000 \mathrm{cell} / \mathrm{cm}^{2}$ at passage 3 unless stated otherwise.

\section{Immunocytochemistry}

After cell culture, the cells were washed with phosphate buffered saline (PBS; Merck) and fixed with $4 \%(\mathrm{w} / \mathrm{v})$ paraformaldehyde (Sigma-Aldrich) for $5 \mathrm{~min}$ at $37^{\circ} \mathrm{C}$. After washing three times, cells were permeabilized with $0.01 \%$ (v/v) Triton X-100 (Acros Organics) and blocked with goat serum (1:100; Sigma-Aldrich) in PBT (PBS $+0.02 \%$ Triton-X-100, 0.5\% BSA) for $1 \mathrm{~h}$. Afterwards, cells were incubated with the primary antibody dissolved in PBT for $1 \mathrm{~h}$. Cells were washed three times and incubated with goat anti-rabbit secondary antibody conjugated to Alexa Fluor 647 (1:500; ThermoFisher), together with Phalloidin conjugated to Alexa Fluor 568 (1:500; ThermoFisher) in PBT for $1 \mathrm{~h}$. After washing, the nucleus was counterstained with Hoechst 33258 (1:1000; Sigma-Aldrich) for $20 \mathrm{~min}$. After washing three times, surfaces were mounted on glass cover slides with mounting media (Dako). All washing steps were performed with PBT. Primary antibodies used in this study were: anti-Scx antibody (1:200; Abcam; ab58655) and antiEgr1 antibody (1:200; Thermo Fisher Scientific; T.126.1). For live cell imaging, Celltracker Green CMDFA (Thermo Fisher Scientific) was used according to manufacturer's instructions.

\section{Image Analysis}

Fixed samples were inverted and fluorescent images were acquired through the glass coverslip using a fully automated Nikon Eclipse Ti-U microscope in combination with an Andor Zyla 5.5 4MP camera. For live cell imaging, the same microscopy setup was applied, yet whereby cells were held inside an incubation chamber at $37{ }^{\circ} \mathrm{C}$ and a $5 \% \mathrm{CO}_{2}$ atmosphere. Images were 
analyzed through CellProfiler ${ }^{47}$ with custom-made pipelines. After illumination corrections, nuclei morphology was captured using the Otsu adaptive thresholding method applied on the Hoechst 33258 channel. Subsequently, cell morphology was determined by applying Otsu adaptive thresholding and appropriate propagation algorithms on the Phalloidin channel. To prevent the influence of the walls on the cell morphology measurements, cells touching the walls were filtered out of the dataset. After background correction, Scleraxis integrated intensity values were calculated based on the Scleraxis staining inside the nuclear area.

\section{Machine learning algorithms to associate SCX levels with surface features and cell morphology}

To identify surface design parameters that influence SCX expression, the 200 surfaces with the highest SCX expression and the 200 surfaces with the lowest SCX expression were selected. To classify the topographies that had a positive or negative effect on the integrated Intensity of the SCX signal, a classification tree algorithm from "rpart" package ver. 4.11 that was run in R ver. 3.3.3 ${ }^{62}$. The classification tree was visualized by this "partykit" package ver. $1.0^{63}$. The Random Forest algorithm was used to create the predictive model. The accuracy was depicted by the receiver operating curve (ROC), which illustrates the performance of the binary classifier by plotting the true positive rate, against the false positive rate, at various threshold settings. In order to have a training set for testing accuracy of the model, the data set was split into 2 parts. The first part contained $75 \%$ of the data and was used for model training and the remaining $25 \%$ was used for model testing. The models were trained with 10 fold cross validation in "caret" package version 6.0.

\section{Quantitative PCR (RT-qPCR)}

Total RNA was isolated by using the RNeasy Mini Kit (Qiagen) according to manufacturer's protocol. Reverse transcription was carried out using an iScript ${ }^{\mathrm{TM}} \mathrm{CDNA}$ synthesis kit (Bio-Rad). Quantitative PCR was performed using the $\mathrm{iQ}^{\mathrm{TM}}$ SYBR ${ }^{\circledR}$ Green Supermix (Bio-Rad) in a CFX96 ${ }^{\text {TM }}$ Real-Time PCR Detection Kit (Bio-Rad). Glyceraldehyde 3-phosphate dehydrogenase (GAPDH) was used as a housekeeping gene and relative expression was determined using the $\Delta \Delta \mathrm{Ct}$ method. Primer sequences are listed in Supplementary Data File 3.

\section{Statistical analysis}

Statistical significance of integrated SCX intensity values between flat and surface microtopographies was determined by Dunns post-hoc test. A p-value smaller than 0.05 after Benjamini and Hochberg correction for multiple testing is considered significant. Statistical significance of RT-qPCR experiments is determined by one sample t-test after log transformation of expression fold changes. 


\section{Acknowledgments}

$\mathrm{SV}, \mathrm{AV}, \mathrm{AD}, \mathrm{NR}, \mathrm{PV}, \mathrm{AD}$ and $\mathrm{JdB}$ acknowledge the financial support of the Dutch province of Limburg, AV the European Union's Seventh Framework Programme (FP7/ 2007-2013; Grant agreement 289720), SV, DS and AD the European Union's Horizon 2020 Programme (H2020-MSCA- ITN-2015; Grant agreement 676338).

\section{Disclosure Statement}

$\mathrm{JdB}$ is a co-founder of Materiomics b.v. The other authors do not have any financial interests to declare.

\section{References}

1. Clayton, R. A. E. \& Court-Brown, C. M. The epidemiology of musculoskeletal tendinous and ligamentous injuries. Injury 39, 1338-1344 (2008).

2. Sharma, Pankaj, N. M. Tendon Injury and Tendinopathy: Healing and Repair. J. Bone Jt. Surg. 87,187 (2005).

3. Hirsch, T. et al. Regeneration of the entire human epidermis using transgenic stem cells. Nat. Publ. Gr. 551, 327-332 (2017).

4. Mazzocca, A. D. et al. In vitro changes in human tenocyte cultures obtained from proximal biceps tendon: Multiple passages result in changes in routine cell markers. Knee Surgery, Sport. Traumatol. Arthrosc. 20, 1666-1672 (2012).

5. Pauly, S. et al. Characterization of tendon cell cultures of the human rotator cuff. Eur. Cells Mater. 20, 84-97 (2010).

6. Yao, L., Bestwick, C. S., Bestwick, L., Maffulli, N. \& Aspden, R. M. Phenotypic drift in human tenocyte culture. Tissue Eng. 12, 1843-1849 (2006).

$7 . \quad$ Holtzer, H., Abbot, J., Lash, J. \& S., H. The Loss of Phenotypic Traits by Differentiated Cells in vitro, I. Dedifferentiation of Cartilage Cells. PNAS 46, 1533-1542 (1960).

8. Schiltz, J. R., Mayne, R. \& Holtzer, H. The Synthesis of Collagen and Glycosaminoglycans by Dedifferentiated Chondroblasts in Culture. Differentiation 1, 97-108 (1973).

9. Pawelek, J. M. Effects of thyroxin and low oxygen tension on chondrogenic expression in cell culture. Dev. Biol. 19, 52-72 (1969).

10. Benya, P. D. \& Shaffer, J. D. Dedifferentiated chondrocytes reexpress the differentiated collagen phenotype when cultured in agarose gels. Cell 30, 215-224 (1982).

11. Yaeger, P. C. et al. Synergistic action of transforming growth factor- $\beta$ and insulin-like growth factor-I induces expression of type II collagen and aggrecan genes in adult human articular chondrocytes. Exp Cell Res 237, 318-325 (1997).

12. Siddappa, R. et al. cAMP/PKA pathway activation in human mesenchymal stem cells in vitro results in robust bone formation in vivo. Proc. Natl. Acad. Sci. U. S. A. 105, 7281-7286 (2008).

13. Gilbert, P. et al. Substrate elasticity regulates skeletal muscle stem cell self-renewal in culture. Science (80-. ). 329, 1078-1081 (2011).

14. Tang, J. B., Xu, Y., Ding, F. \& Wang, X. T. Tendon healing in vitro: Promotion of collagen gene expression by bFGF with NF-xB gene activation. J. Hand Surg. Am. 28, 215-220 (2003).

15. Thomopoulos, S., Harwood, F. L., Silva, M. J., Amiel, D. \& Gelberman, R. H. Effect of several growth factors on canine flexor tendon fibroblast proliferation and collagen synthesis in vitro. J. Hand Surg. Am. 30, 441-447 (2005).

16. Webb, S., Gabrelow, C., Pierce, J., Gibb, E. \& Elliott, J. Retinoic acid receptor signaling preserves tendon stem cell characteristics and prevents spontaneous differentiation in vitro. Stem Cell Res. Ther. 7, 45 (2016).

17. Heinemeier, K. M. \& Kjaer, M. In vivo investigation of tendon responses to mechanical loading. J. Musculoskelet. Neuronal Interact. 11, 115-123 (2011). 
18. Zhang, J. \& H-C Wang, J. The Effects of Mechanical Loading on Tendons -An In Vivo and In Vitro Model Study. PLoS One 8, (2013).

19. Kayama, T. et al. Gtf2ird1-Dependent Mohawk Expression Regulates Mechanosensing properties of the tendon. Mol. Cell. Biol. 36, 1297-1309 (2016).

20. Chen, C. S. et al. Geometric Control of Cell Life and Death. 276, 1425-1428 (1997).

21. Connelly, J. T. et al. Actin and serum response factor transduce physical cues from the microenvironment to regulate epidermal stem cell fate decisions. Nat. Cell Biol. 12, 711-718 (2010).

22. Dalby, M. J. et al. The control of human mesenchymal cell differentiation using nanoscale symmetry and disorder. Nat. Mater. 6, 997-1003 (2007).

23. Markert, L. D. et al. Identification of Distinct Topographical Surface Microstructures Favoring Either Undifferentiated Expansion or Differentiation of Murine Embryonic Stem Cells. Stem Cells Dev. 18, 1331-1342 (2009).

24. Biggs, M. J. P. et al. The use of nanoscale topography to modulate the dynamics of adhesion formation in primary osteoblasts and ERK/MAPK signalling in STRO-1+ enriched skeletal stem cells. Biomaterials 30, 5094-5103 (2009).

25. Seo, C. H., Furukawa, K., Montagne, K., Jeong, H. \& Ushida, T. The effect of substrate microtopography on focal adhesion maturation and actin organization via the RhoA/ROCK pathway. Biomaterials 32, 9568-9575 (2011).

26. Schwachtgen, J. L., Houston, P., Campbell, C., Sukhatme, V. \& Braddock, M. Fluid shear stress activation of egr-1 transcription in cultured human endothelial and epithelial cells is mediated via the extracellular signal-related kinase 1/2 mitogen-activated protein kinase pathway. J. Clin. Invest. 101, 25402549 (1998).

27. Liu, Y., Suzuki, Y. J., Day, R. M. \& Fanburg, B. L. Rho kinase-induced nuclear translocation of ERK1/ERK2 in smooth muscle cell mitogenesis caused by serotonin. Circ. Res. 95, 579-586 (2004).

28. Guerquin, M. J. et al. Transcription factor EGR1 directs tendon differentiation and promotes tendon repair. J. Clin. Invest. 123, 3564-3576 (2013).

29. Kannus, P. Structure of the tendon connective tissue. Scand. J. Med. Sci. Sport. 10, 312-320 (2000).

30. Eriksen, H. a, Pajala, A., Leppilahti, J. \& Risteli, J. Increased content of type III collagen at the rupture site of human Achilles tendon. J. Or 20, 1352-1357 (2002).

31. Mackie, E. J. \& Ramsey, S. Expression of tenascin in joint-associated tissues during development and postnatal growth. J. Anat. 188 ( Pt 1, 157-65 (1996).

32. Järvinen, $\mathrm{T}$. et al. Mechanical loading regulates tenascin- $\mathrm{C}$ expression in the osteotendinous junction. J. Cell Sci. 112 Pt 18, 3157 (1999).

33. Zhang, G. et al. Decorin regulates assembly of collagen fibrils and acquisition of biomechanical properties during tendon development. J. Cell. Biochem. 98, 1436-1449 (2006).

34. LeAnn M. Dourte, Lydia Pathmanathan, Michael J. Mienaltowski, A. F. J. \& David E. Birk, L. J. S. Mechanical, Compositional, and Structural Properties of the Mouse Patellar Tendon with Changes in Biglycan Gene Expression. J Orhop Res. 29, 1883-1889 (2008).

35. Andrew A. Dunkmana, Mark R. Buckleya, Michael J. Mienaltowskib, Sheila M. Adamsb, Stephen J. Thomasa, Lauren Satchella, Akash Kumara, Lydia Pathmanathana, D. P. \& Beasona, Renato V. Iozzoc, David E. Birkb, L. J. S. The Tendon Injury Response Is Influenced by Decorin and Biglycan. Ann Biomed Eng. 42, 619-630 (2012).

36. Docheva, D., Hunziker, E. B., Fassler, R. \& Brandau, O. Tenomodulin Is Necessary for Tenocyte Proliferation and Tendon Maturation. Mol. Cell. Biol. 25, 699-705 (2005).

37. Murchison, N. D. et al. Regulation of tendon differentiation by scleraxis distinguishes forcetransmitting tendons from muscle-anchoring tendons. Development 134, 2697-2708 (2007).

38. Liu, W. et al. The Atypical Homeodomain Transcription Factor Mohawk Controls Tendon Morphogenesis. Mol. Cell. Biol. 30, 4797-4807 (2010).

39. Gaut, L. et al. EGR1 regulates transcription downstream of mechanical signals during tendon formation and healing. PLoS One 11, 1-16 (2016).

40. Mendias, C. L., Gumucio, J. P., Bakhurin, K. I. \& Evan, B. Physiological Loading of Tendons Induces Scleraxis Expression in Epitenon Fibroblasts. J Orthop Res 30, 606-612 (2012).

41. Léjard, V. et al. Scleraxis and NFATc regulate the expression of the pro- $\alpha 1(\mathrm{I})$ collagen gene in tendon fibroblasts. J. Biol. Chem. 282, 17665-17675 (2007). 
42. Shukunami, C., Takimoto, A., Oro, M. \& Hiraki, Y. Scleraxis positively regulates the expression of tenomodulin, a differentiation marker of tenocytes. Dev. Biol. 298, 234-247 (2006).

43. Killian, M. L., Cavinatto, L., Galatz, L. M. \& Thomopoulos, S. The role of mechanobiology in tendon healing. J Shoulder Elb. Surg. (2012). doi:10.1016/j.jse.2011.11.002

44. Unadkat, H. V et al. An algorithm-based topographical biomaterials library to instruct cell fate. Proc. Natl. Acad. Sci. U. S. A. 108, 16565-70 (2011).

45. Hulshof, F. F. B. et al. Mining for osteogenic surface topographies: In silico design to in vivo osseo-integration. Biomaterials 137, 49-60 (2017).

46. Bach. Le, A. Vasilevich, S. Vermeulen, F. Hulshof, F. Stamatialis, C. van Blitterswijk, J. de B. Micro-Topographies Promote Late Chondrogenic Differentiation Markers in the ATDC5 Cell Line. Tissue Eng. Part A 23, 458-469 (2017).

47. Carpenter, A. E. et al. CellProfiler: image analysis software for identifying and quantifying cell phenotypes. Genome Biol. 7, R100 (2006).

48. English, A. et al. Substrate topography: A valuable in vitro tool, but a clinical red herring for in vivo tenogenesis. Acta Biomater. 27, 3-12 (2015).

49. Kapoor, A., Caporali, E. H. G., Kenis, P. J. A. \& Stewart, M. C. Microtopographically patterned surfaces promote the alignment of tenocytes and extracellular collagen. Acta Biomater. 6, 2580-2589 (2010).

50. Zhu, J. et al. The regulation of phenotype of cultured tenocytes by microgrooved surface structure. Biomaterials 31, 6952-6958 (2010).

51. $\mathrm{Xu}, \mathrm{B}$. et al. RhoA/ROCK, cytoskeletal dynamics, and focal adhesion kinase are required for mechanical stretch-induced tenogenic differentiation of human mesenchymal stem cells. J. Cell. Physiol. 227, 2722-2729 (2012).

52. Maharam, E. et al. Rho/Rock signal transduction pathway is required for MSC tenogenic differentiation. Bone Res. 3, 15015 (2015).

53. Zuckerbraun, B. S., Shapiro, R. A., Billiar, T. R. \& Tzeng, E. RhoA influences the nuclear localization of extracellular signal-regulated kinases to modulate $\mathrm{p} 21 \mathrm{Waf} / \mathrm{Cip} 1$ expression. Circulation 108, 876-881 (2003).

54. Tavares, S. et al. Actin stress fiber organization promotes cell stiffening and proliferation of preinvasive breast cancer cells. Nat. Commun. 8, (2017).

55. Eliasson, P., Andersson, T., Hammerman, M. \& Aspenberg, P. Primary gene response to mechanical loading in healing rat Achilles tendons. J. Appl. Physiol. 114, 1519-1526 (2013).

56. Spaapen, F. et al. The Immediate Early Gene Product EGR1 and Polycomb Group Proteins Interact in Epigenetic Programming during Chondrogenesis. PLoS One 8, (2013).

57. Mace, C. R. et al. Loss of transcription factor early growth response gene 1 results in impaired endochondral bone repair. Bone 49, 743-752 (2011).

58. Liu, C.-F. \& Lefebvre, V. The transcription factors SOX9 and SOX5/SOX6 cooperate genomewide through super-enhancers to drive chondrogenesis. Nucleic Acids Res. 43, 8183-8203 (2015).

59. Buckley, M. R. et al. Distributions of types I, II and III collagen by region in the human supraspinatus tendon. Connect. Tissue Res. 54, 374-379 (2013).

60. Sugimoto, Y. et al. Scx+/Sox $9+$ progenitors contribute to the establishment of the junction between cartilage and tendon/ligament. Development 140, 2280-8 (2013).

61. Zhao, Y. et al. High-definition micropatterning method for hard, stiff and brittle polymers. Mater. Sci. Eng. C 71, 558-564 (2017).

62. R Core Team. R: A language and environment for statistical computing. R Found. Stat. Comput. 63. Hothorn, T., Hornik, K. \& Zeileis, A. Unbiased Recursive Partitioning: A Conditional Inference Framework. J. Comput. Graph. Stat. 15, 651-674 (2006). 


\section{Supplementary Figures}

\section{a}

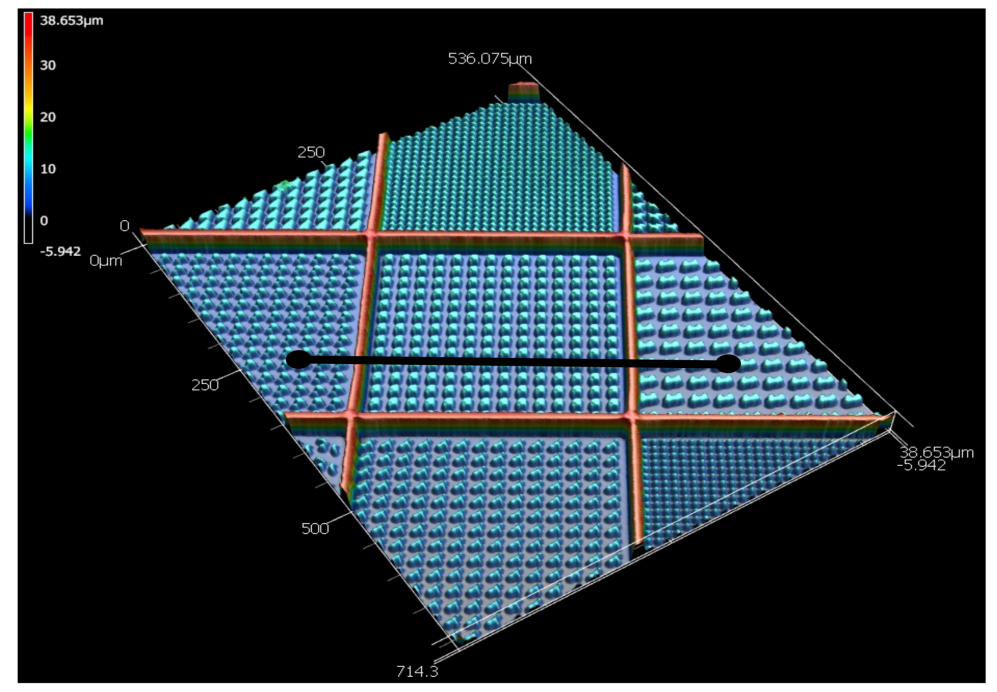

b

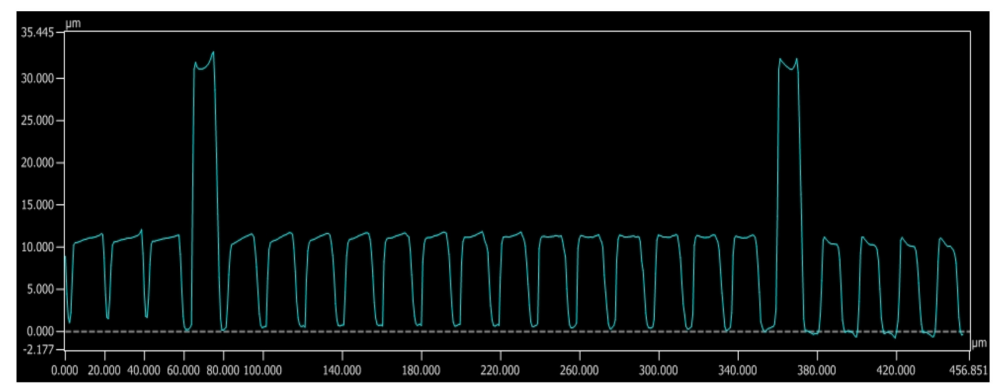

Supplementary Figure 1: Optical profilometric imaging of the TopoChip platform. a) 3D reconstruction reveals the wall and feature structures of the TopoChip. The black line represents cross-section taken for measuring the height profile. b) The height profile confirms a $10 \mu \mathrm{m}$ height of the feature structures and a $30 \mu \mathrm{m}$ height of the walls dividing the individual TopoUnits in a 290x290 $\mu \mathrm{m}$ area. 


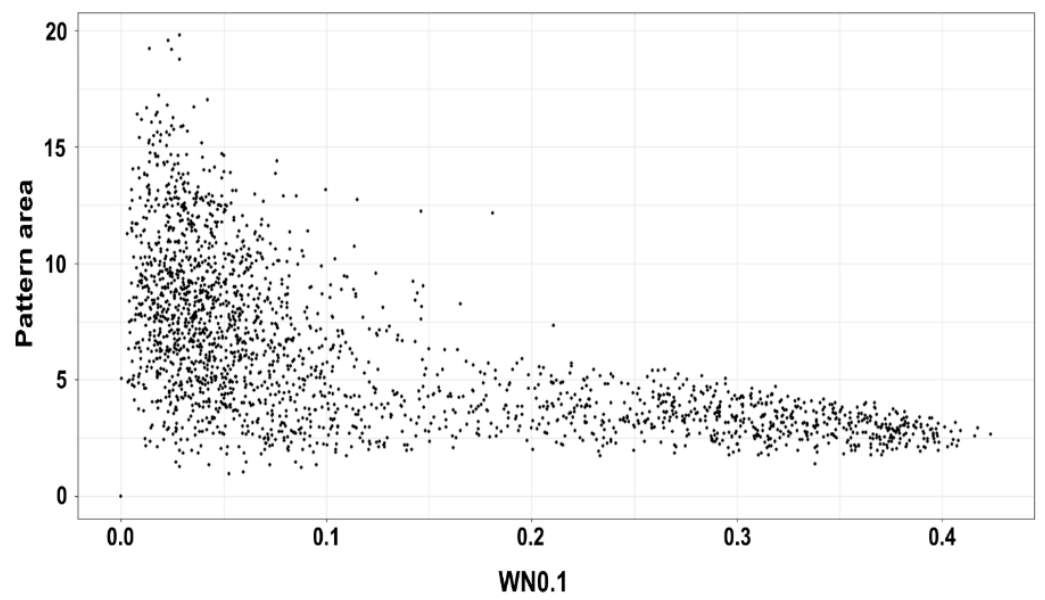

Supplementary Figure 2: Dot plot representation of WN0.1 and pattern area of all 2176 micro-topographical architectures on the TopoChip. The flat surface is represented by a WN0.1 and pattern area of 0 . Higher micro-topographical pattern areas are only allowed with lower WN0.1.

a

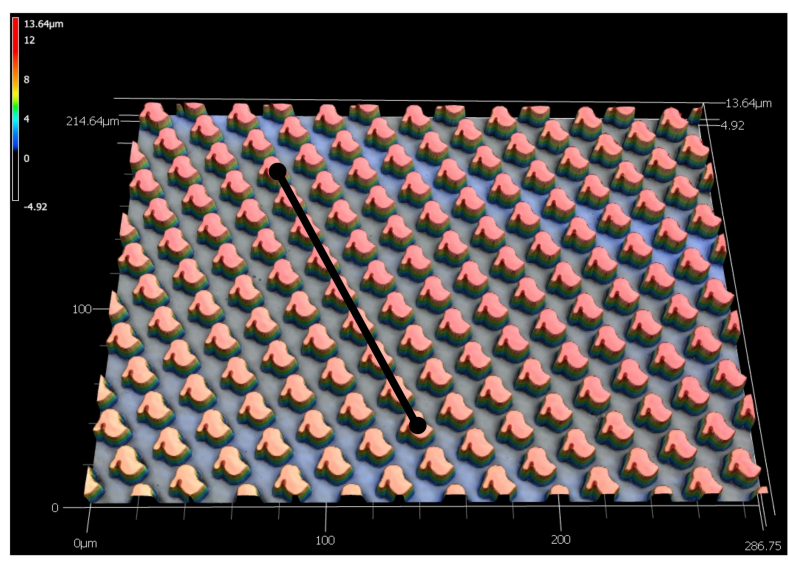

Supplementary Figure 3: Optical profilometric imaging of the PS0304 surface. a) $3 \mathrm{D}$ reconstruction reveals the feature architecture of PS0304. The black line represents crosssection taken for measuring the height profile. b) The height profile confirms a $10 \mu \mathrm{m}$ height of the PS0304 feature structures with a side length of $20 \mu \mathrm{m}$.

b




a

48 hours

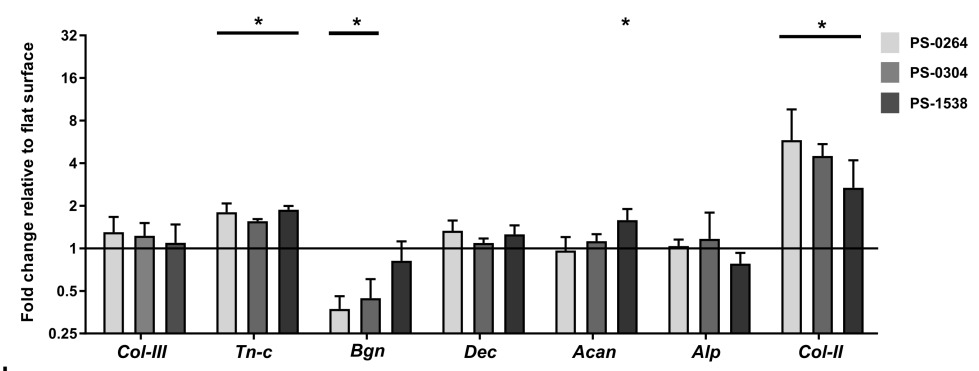

b

7 days

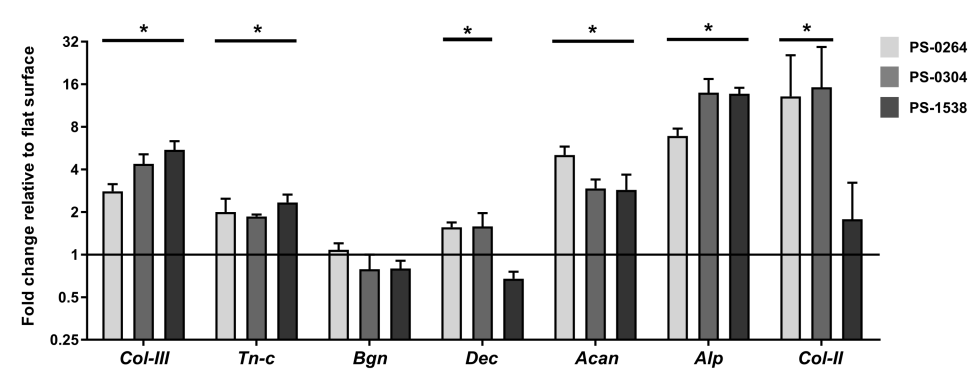

Supplementary Figure 5: Bar chart representation of gene expression levels of tendon-related matrix genes and the osteogenic marker $A l p$ and the chondrogenic marker Col-II. Tenocytes were cultured on PS-Flat, PS-0264, PS0304 and the PS-1538 surface (* $\mathrm{P}<0.05)$.

a

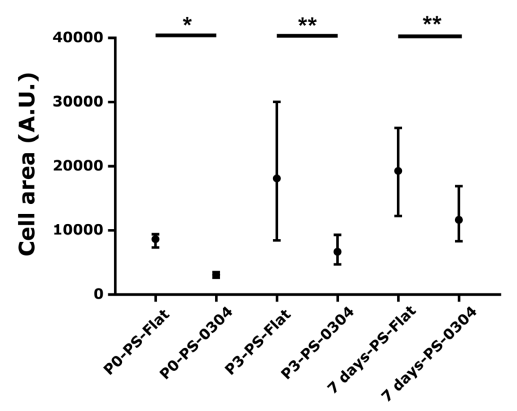

b

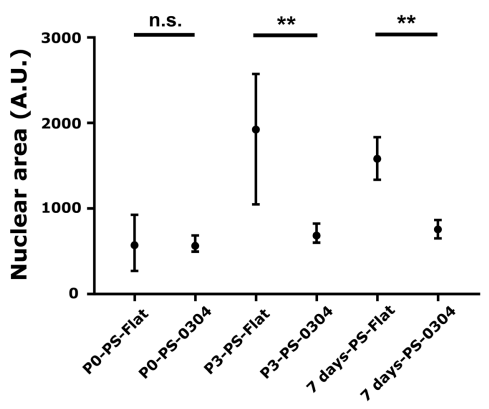

Supplementary Figure 6: a) Quantification of cell area reveals that cells grown on PS0304 had smaller spreading compared to cells cultured on flat and similar levels as P0 tenocytes on flat $(* \mathrm{P}<0.05 ; * * \mathrm{P}<0.001)$. b) Quantification of the nuclear area reveals that cells grown on PS-0304 obtained smaller nuclear dimensions compared to cells grown on the flat surface $(* \mathrm{P}<0.001)$ and similar levels as P0 tenocytes. Median is represented by a black dot. Error bars represent interquartile range. 


\section{Chapter V}

\section{Mechanotransduction is a Context-}

\section{Dependent Activator of TGF- $\beta$}

\section{Signaling in Mesenchymal Stem Cells}

Steven Vermeulen ${ }^{1,2}$, Nadia Roumans ${ }^{1}$, Floris Honig ${ }^{1}$, Aurélie Carlier ${ }^{1}$, Dennie G.A.J. Hebels ${ }^{1}$, Aysegul Dede Eren ${ }^{1,2}$, Peter ten Dijke ${ }^{3}$, Aliaksei Vasilevich ${ }^{2}$, Jan de Boer ${ }^{2, *}$

${ }^{1}$ Laboratory for Cell Biology-Inspired Tissue Engineering, MERLN Institute, University of Maastricht, Maastricht, The Netherlands

${ }^{2}$ BioInterface Science Group, Department of Biomedical Engineering and Institute for Complex Molecular Systems, University of Eindhoven, Eindhoven, The Netherlands.

${ }^{3}$ Oncode Institute and Dept. Cell and Chemical Biology, Leiden University Medical Center, Leiden, The Netherlands

Submitted for publication 


\section{Abstract}

We previously found that surface topographies induce the expression of the Scxa gene, encoding Scleraxis in tenocytes. Because $S c x a$ is a TGF- $\beta$ responsive gene, we investigated the link between mechanotransduction and TGF- $\beta$ signaling. We discovered that mesenchymal stem cells exposed to both micro-topographies and TGF- $\beta 2$ display synergistic induction of SMAD phosphorylation and transcription of the TGF- $\beta$ target genes SCXA, a-SMA, and SOX9. Pharmacological perturbations revealed that Rho/ROCK/SRF signaling is required for this synergistic response. We further found an activation of the early response genes $S R F$ and EGR1 during the early adaptation phase on micro-topographies, which coincided with higher expression of the TGF- $\beta$ type-II receptor gene. Of interest, PKC activators Prostratin and Ingenol-3, known for inducing actin reorganization and activation of serum response elements, were able to mimic the topography-induced TGF- $\beta$ response. These findings provide novel insights into the convergence of mechanobiology and TGF- $\beta$ signaling, which can lead to improved culture protocols and therapeutic applications. 


\section{Introduction}

Under physiological conditions, tissue homeostasis is maintained by the appropriate spatiotemporal responses of cells to environmental signals, such as secreted cytokines and transmembrane proteins from adjacent cells, but also by mechanical forces and changes in cell shape ${ }^{1}$. The latter is evident by the secretion and autocrine activity of insulin-like growth factor (IGF) by myocytes upon mechanical stimulation, a potent growth factor that induces muscle growth ${ }^{2}$. Similarly, tendon tissue homeostasis and growth depends on both mechanical forces ${ }^{3}$ and transforming growth factor (TGF- $\beta$ ) signaling ${ }^{4}$. Also, the myofibroblastic state during tissue repair depends both on the mechanical characteristics of the matrix environment as well as the presence of TGF- $\beta^{5}$. During embryonic development, mechanical forces are essential for proper morphogenesis in conjunction with biochemical signals, as shown by the spatial reorganization of TGF- $\beta$ receptors upon cell confinement in gastruloids ${ }^{6}$. Mechanical forces can rapidly activate various intracellular signaling pathways ${ }^{7}$. Well-documented examples include the activation of the transcription factors yes-associated protein 1 (YAP), and transcriptional coactivator with PDZ-binding motif (TAZ) through stretching ${ }^{8}$ and cell shape changes 9 . These transcription factors are essential for tissue homeostasis and embryogenic processes ${ }^{10,11}$, such as osteogenesis ${ }^{12}$, which is also influenced by bone morphogenetic protein 2 (BMP-2) ${ }^{13}$. Changes in actin dynamics influence serum response factor (SRF) activity through altered binding with co-transcription factors ${ }^{14}$, leading to broad changes in physiological processes ${ }^{15,16}$, including myofibroblast differentiation ${ }^{17}$ which in turn can be regulated through TGF- $\beta$ signaling ${ }^{18}$. How these mechanical and biochemical signals converge to drive cell behavior is poorly understood. Therefore, gaining novel insights in these mechanisms is vital for developing therapeutics in case of improper cell function and tissue engineering applications.

Since it is difficult to decouple the effects of biochemical and mechanical stimuli in vivo, essential insights are gained by in vitro experiments. Here, physical cues relayed through altered surface geometry can offer mechanical stimulation through changing cell shape. Cell geometry profoundly affects cell behavior, as shown by altering the lineage specification of stem cells ${ }^{19-}$ 21. Evidence exists that cell geometry also influences the biological effects of soluble factors. For example, adhesive islands alter the genomic response after tumor necrosis factor (TNF) $\alpha$ stimulation ${ }^{22}$, and growth factor signaling from BMP-2 ${ }^{23}$ or serum ${ }^{24}$. Also, cell confinement through micro-wells reduces the inflammatory response to lipopolysaccharide (LPS) in macrophages ${ }^{25}$. Of interest here is that surprisingly little is known how surface topography controls the cell's response towards growth factor signaling. This is an important consideration since growth factors are involved in numerous biological processes, including differentiation 26 and maintenance of phenotypic identity ${ }^{27}$. Research involving cell stretching hints towards an interesting interplay between biomechanical forces and soluble factors. For example, cell stretching increases the sensitivity for soluble factors through altering receptor expression or activity ${ }^{28-30}$. In this manuscript, we provide new insights in this field by demonstrating that topographical cues alter the response of mesenchymal stem cells (MSCs) to TGF- $\beta$ signaling. 


\section{Results}

\section{MSCs cultured on micro-topographies display altered actin dynamics and differential expression of cytoskeletal genes}

An eye-catching characteristic of cells cultured on surface structures are profound changes in cell morphology ${ }^{31,32}$. We seeded adipose-derived MSCs on micro-topography PS-281 (Fig. 1A), and after $24 \mathrm{~h}$ observed changes in shape accompanied by a reduction of filamentous(F)-actin stress fibers compared to MSCs cultured on a flat surface (Fig. 1B-C). We previously assessed the transcriptome of bone marrow-derived MSCs on surface PS-281 relative to MSCs on flat control surfaces (manuscript in preparation) and produced a STRING protein-protein interaction network with 248 nodes and 1839 edges based on differentially expressed genes (DEGs) with a fold change $>1.5$ and adjusted $\mathrm{p}$-value $<0.05$. The network includes early growth response gene 1 (EGR1) (Fig. 1D-E; 3.0 fold change), FBJ murine osteosarcoma viral oncogene homolog (FOS) (4.7 fold change), and FOSB (2.2 fold change), all of which are mechanosensitive transcription factors ${ }^{33}$. We next produced a volcano plot of genes with Gene Ontology (GO) term "cytoskeleton organization", and noticed that many cytoskeleton related DEGs exhibit lower expression when cultured on surface PS-281, with a total of 48 DEGs downregulated and 11 DEGs upregulated (Fig. 1F and Supplementary Table 1 and 2). Downregulated genes are involved in microtubule dynamics, e.g., stathmin 1 (STMN1; -1.6 fold change), an important cytoskeletal effector regulating microtubule dynamics ${ }^{34}$, tubulin $\beta$ class I (TUBB; -1.9 fold change), tubulin $\beta$ 4B class IVb (TUBB4B; -1.8 fold change), tubulin $\alpha 1 \mathrm{a}$ (TUBA1A; -1.8 fold change), and tubulin $\beta 6$ class V (TUBB6; -1.6 fold change). Other genes such as actin $\gamma-1$ (ACTG1; -1.9 fold change) and tropomyosin 3 (TPM3; -1.7 fold change) form integral parts of the cytoskeleton ${ }^{35}$. Of interest, we also observed a slight downregulation of actin $\beta$ (ACTB; -1.3 fold change). We further found a reduction in expression levels of genes associated with small GTPase rho signal transduction and subsequent cytoskeletal organization ${ }^{36}$ (Supplementary Fig. 1). For example, we detected a downregulation of ezrin (EZR; -1.9 fold change), an actinbinding protein that acts as a linker between the actin cytoskeleton and plasma membrane proteins ${ }^{37}$, and positively modulates rho signaling through the interaction with rho GDP dissociation inhibitors ${ }^{38}$. A downregulation was observed for diaphanous related formin 3 (DLAPH3; -1.5 fold change), which is required for $\mathrm{F}$-actin stress fiber formation ${ }^{39}$ and regulates SRF activity ${ }^{40}$. We also mention the downregulation of anillin actin-binding protein (ANLN; 1.7 fold change), which is important for cytoskeletal dynamics ${ }^{41}$ and is involved in rho signaling 42. The gene signature induced by surface PS-281 demonstrates that the cytoskeleton is under a lot of change $24 \mathrm{~h}$ after cell seeding, which corresponds with the visual observed alterations in cytoskeleton architecture and cell geometry. 


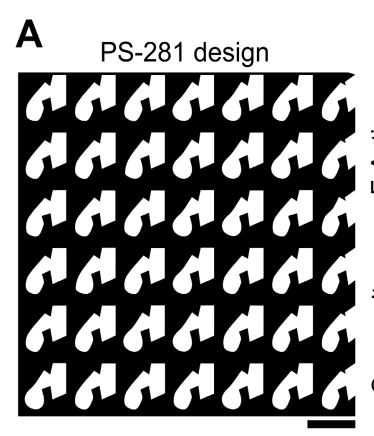

D

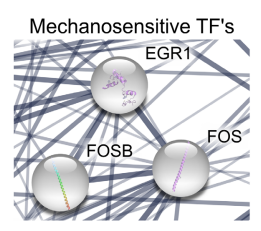

E

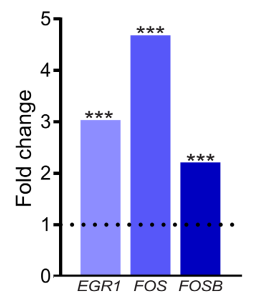

$\mathbf{F}$

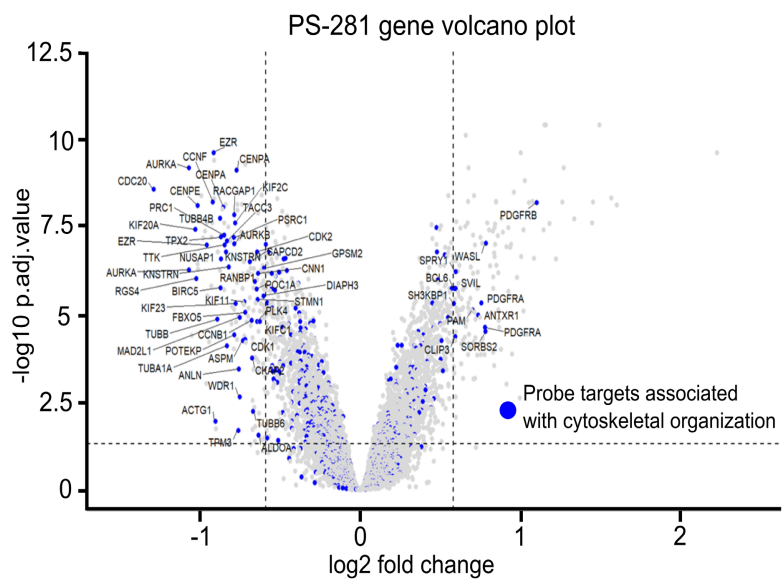

Figure 1: MSCs cultured on micro-topographies display altered actin organization and differential expression of cytoskeletal genes. A) In silico design of the PS-281 micro-topographical surface. Scale bar represent $10 \mu \mathrm{m}$. B) MSCs cultured on a regular PS flat surface exhibit spread morphological characteristics and a profound presence of F-actin stress fibers. C) MSCs cultured on surface PS-281 exhibit elongated and smaller nuclear and cellular characteristics, which coincides with a reduction of F-actin stress fibers. F-actin was immunolabeled with phalloidin (yellow) and the nucleus counterstained with Hoechst33342 (magenta). Scale bars represent $50 \mu \mathrm{m}$. D) Partial representation of a STRING gene network based on a microarray study of MSCs cultured on the PS-281 surface for 24h. The mechanosensitive transcription factors EGR1, FOS, and FOSB are represented here. E) EGR1, FOS, and FOSB expression levels have increased 2 to 5 fold on the PS-281 surface compared to a flat surface $(* * * \mathrm{P}<0.001)$. F) Volcano plot representation of the PS-281 microarray data. Blue dots are probe targets associated with cytoskeletal organization. Majority of these DEGs exhibit lower expression levels compared to flat. DEG cut-off is determined at a 1.5 fold change and an adjusted P value of 0.05 .

\section{Activation of early response genes is associated with early actin reorganization}

Based on the observed increased expression of FOS and EGR1 at 24h, we decided to investigate actin organization dynamics and the expression of early genes and proteins with a known relation to actin remodeling on surface PS-1018, which we previously discovered as a surface that induces Scleraxis $(S c x)$ expression in tenocytes ${ }^{43}$. Furthermore, PS-1018 can be manufactured in a 100 $\mathrm{mm}$ dish format, thereby facilitating downstream experiments. Surface PS-1018 (Fig. 2A) 
induced cell elongation with a profound reduction in cell, and nuclear size, while reducing actin stress fibers (Fig. 2B), similar to surface PS-281. As early as 1 h after cell seeding, we noted that cells on flat surfaces exhibited a diffuse actin pattern with a round cell morphology (Fig. 2C); however, MSCs adhering on the surface PS-1018 displayed different dynamics. Here, MSCs engulfed the micro-topographies with concentrated F-actin stress fiber formation on top of the structures (Fig. 2D), but a more diffuse actin pattern at the bottom and in between the structures (Fig. 2E). Quantification of F-actin levels showed significantly elevated F-actin levels at $1 \mathrm{~h}$ and 2h on PS-1018, with levels peaking at $1 \mathrm{~h}$ and dropping afterward (Supplementary Fig. 2A-B). To probe the early regulatory responses, we exposed MSCs cultured for $2 \mathrm{~h}$ to flat and surface PS-1018 and analyzed the phospho-proteome by mass spectrometry (Supplementary Fig. 3). We detected increased levels of phosphorylated actin in cells cultured on surface PS-1018, indicating active cytoskeletal reorganization ${ }^{44,45}$. Also, we detected increased levels of phosphorylated adenylyl cyclase-associated protein 1 (CAP-1) and drebrin 1 (DBN1), which regulate actin dynamics ${ }^{46,47}$. These observations indicate that MSCs cultured on the microtopography are subjected to dynamic cytoskeletal regulation, characterized by an early adaptation phase involving actin remodeling.

We next investigated genes and proteins, of which it is known that their expression or activity is influenced by actin. First, we observed a 3.6 fold increase in EGR1 mRNA levels compared to flat at $24 \mathrm{~h}$ and no significant difference at $48 \mathrm{~h}$ (Fig. 2F). At earlier time points, we assessed EGR1 protein expression dynamics on both flat and PS-1018 (Fig. 2G). At 2h, we found increased EGR1 levels in the nucleus when MSCs are cultured on PS-1018 compared to flat (Fig $\mathbf{2 H}$ ). Of interest, we measured a slight yet non-significant increase at $24 \mathrm{~h}$. This seemingly contradicts the qPCR and microarray observations on surface PS-281; however, we believe this caused by a subpopulation of cells with high EGR1 levels on the PS-1018 surface that skews the global EGR1 levels measured on RNA level. Equally interesting was the observation that after 2h EGR1 levels on a flat surface were higher than on flat and the PS-1018 surface at $24 \mathrm{~h}$. We contribute this phenomenon to cell-seeding that induces a biomechanical response.

Next, we explored if we could detect alterations in SRF levels after $2 \mathrm{~h}$ and 24h on PS-1018 (Fig. 2I). SRF is an important transcription factor that is associated with changes in actin dynamics 42 and known for inducing transcription of genes with serum response elements in its promoter, which includes FOS, EGR1, and SRF itself ${ }^{48,49}$. Similar to EGR1, high intensities of nuclear SRF were observed at $2 \mathrm{~h}$ (Fig. 2J), followed by a slight yet non-significant decrease at $24 \mathrm{~h}$ compared to the flat surface. 


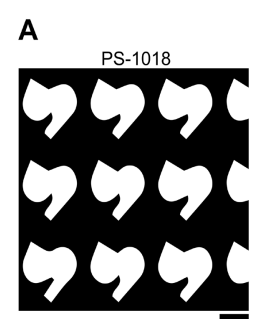

$\mathbf{F}$

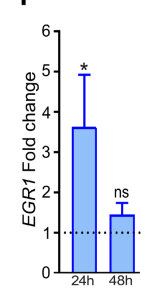

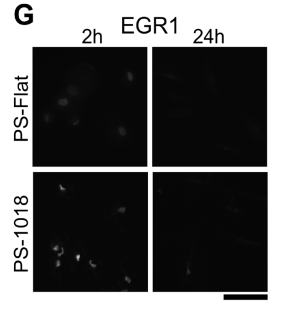

B $\quad$ C
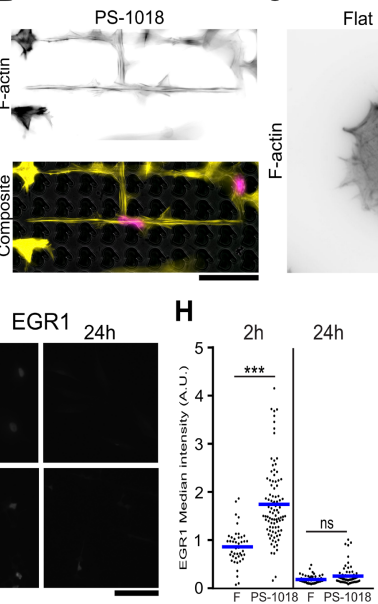

Flat surface $-1 \mathrm{~h}$

D PS-1018-1h-Top view

E PS-1018-1h-Bottom view

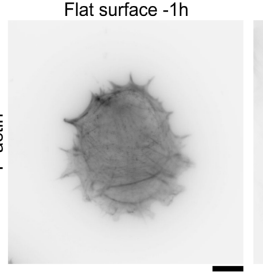
in
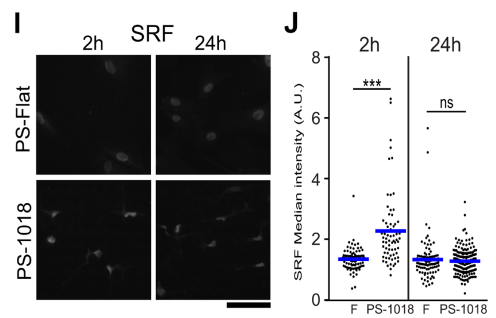

Figure 2: Micro-topographies elevate the early response genes SRF and EGR1 during the cells early adaptation phase. A) In silico design of the PS-1018 surface used in subsequent experiments. Scale bar represent $10 \mu \mathrm{m}$. B) Similar as the PS-281 surface, the PS-1018 surface elicits smaller, elongated morphological characteristics and a reduction in F-actin stress fibers. F-actin was immunolabeled by phalloidin (yellow) and the nucleus counterstained with Hoechst33342 (magenta). Scale bar represent 50 $\mu \mathrm{m}$. C) Immunolabeling of F-actin after $1 \mathrm{~h}$ of cell culture reveals that MSCs on a flat surface exhibit a round morphology with a diffuse F-actin pattern. D-E) Immunolabeling of F-actin of MSCs cultured for $1 \mathrm{~h}$ on the PS-1018 surface reveals an increase in F-actin stress fibers concentrated on the upper part of the micro-topographical structures, while a diffuse pattern is observed at the bottom of the PS-1018 structures. Scale bar represent $10 \mu \mathrm{m}$. F) EGR1 levels are elevated at 24h as measured through qPCR (* $\mathrm{P}<0.05)$, a similar observation as with the PS-281 microarray data. G-H) Immunolabeling of EGR1 demonstrates elevated intensities on the PS-1018 surface at $2 \mathrm{~h}$, with only a few cells on the PS-1018 surface showing elevated levels after 24h. Quantification of EGR1 fluorescent signal confirms the visual observation, with elevated levels measured at $2 \mathrm{~h}(* * * \mathrm{P}<0.001)$, and no significant difference at $24 \mathrm{~h}$. Scale bar represent $100 \mu \mathrm{m}$. I-J) Immunolabeling of SRF demonstrates elevated levels on the PS-1018 surface at $2 \mathrm{~h}(* * * \mathrm{P}<0.001)$, with no significant differences at $24 \mathrm{~h}$. Barplot represent the mean with error bars representing SEM. Dotplots represent intensity levels of individual nuclei. Blue line indicates median. Scale bar represent $100 \mu \mathrm{m}$.

\section{Micro-topographical cues elevate TGF-BR-II and SCX levels in MSCs}

EGR1 elevation on surface PS-1018 is interesting, considering that EGR1 is involved in the expression of the tendon-specific transcription factor scleraxis (SCX) ${ }^{50,51}$, which in previous work was induced in tenocytes on micro-topographies ${ }^{43}$. It is unclear how EGR1 influences SCX but considering that SCX is upregulated in response to TGF- $\beta{ }^{26}$, evidence exists that this is through increased expression of the TGF- $\beta 2$ ligand ${ }^{52}$ or the transforming growth factor- $\beta$ type II receptor (TGF- $\beta$ R-II) ${ }^{53}$. Browsing of the PS-281 transcriptomics data set for TGF- $\beta$ signaling revealed five genes with a significant fold change of more than 1.5 associated with GO biological process "Response to TGF- $\beta$ " (Fig. 3A). These include the previously mentioned FOS gene (4.6 fold change), known for participating with small mothers against decapentaplegic (SMAD) proteins to influence TGF- $\beta$ signaling ${ }^{54}$. Also, an upregulation was observed for the 
TGF- $\beta$ inducible genes Collagen-III (COL-III; 1.6 fold change) ${ }^{55}$, prostate transmembrane protein androgen-induced 1 (PMEPA1; 1.5 fold change) ${ }^{56}$, and matrix remodeling-associated protein 5 (MXRA5; 1.5 fold change) ${ }^{57}$. We also noted a downregulation of neuronal regeneration related protein (NREP; -1.5 fold change), which is related to an expression decrease of the growth factors TGF- $\beta 1$ and TGF- $\beta 2{ }^{58}$. In addition, we noticed an increase in SMAD7 expression (1.41 fold change), an antagonist of the TGF- $\beta$ /SMAD pathway that functions as a negative feedback activator after TGF- $\beta$ signaling ${ }^{59}$. We also mention a 2.81 fold change increase of VCAM1, which is TGF- $\beta$ inducible ${ }^{60}$, yet was not part of the GO list.

Of interest, we also noted (through multiple probes) an upregulation of TGF- $\beta$ R-II (1.9 and 2.0 fold change), an essential component of the TGF- $\beta /$ SMAD signaling pathway. These findings strengthen the hypothesis that micro-topographies sensitize MSCs for TGF- $\beta$ related signaling. We confirmed increased TGF- $\beta$ R-II expression on surface PS-1018 by qPCR after 8 h (Fig. 3B; 1.3 fold change), and reaching a maximum at $24 \mathrm{~h}$ (2.1 fold change). At $48 \mathrm{~h}$, no significant

A
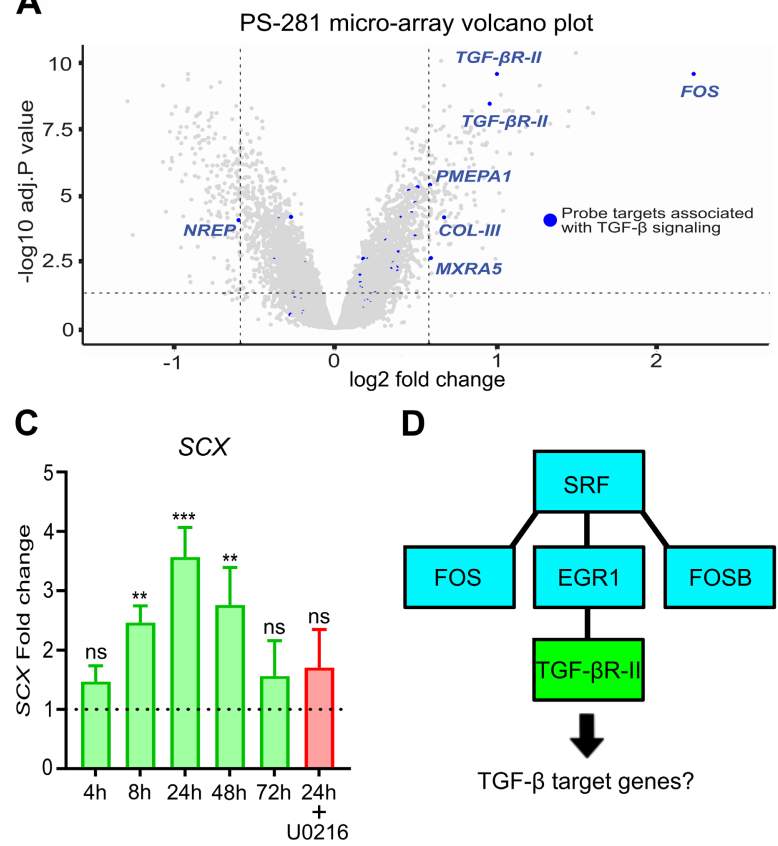

D

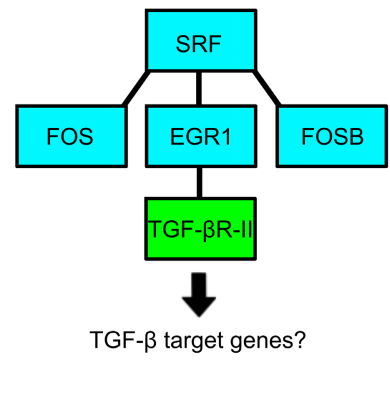

B



Figure 3: Micro-topographies induce elevated levels of TGF- $\beta R-I I$ and the TGF- $\beta$ target gene

SCX. A) Volcano plot of the PS-281 microarray with probe targets associated with TGF- $\beta$ signaling represented in blue. DEG cut-off is determined at a 1.5 fold change and an adjusted $\mathrm{P}$ value of 0.05 . Increased levels of TGF- $\beta$ R-II are observed (1.9 and 2.0 fold increase; *** P<0.001). B) qPCR of MSCs cultured on the PS-1018 surface validates the PS-281 observation regarding TGF- $\beta$ R-II expression, with elevated levels observed at $8 \mathrm{~h}$ and $24 \mathrm{~h}$. The ERK inhibitor U0216 inhibits the topography induced TGF$\beta R-I I$ expression $(* \mathrm{P}<0.05)$. C) The PS-1018 surface induces elevated levels of the TGF- $\beta$ target gene $S C X$. Significant elevated levels were detected at $8 \mathrm{~h}, 24 \mathrm{~h}$ and $48 \mathrm{~h}(* * \mathrm{P}<0.01)$, with a maximum expression at 24h. In addition, the inhibitor U0216 abolishes topography-induced SCX expression. D) Schematic representation of the mechanism involving the activation of SRF and EGR1 leading to an upregulation of TGF- $\beta$ R-II. Barplots represent the mean with error bars representing SEM. 
differences in TGF- $\beta$ R-II levels were detected between PS-1018 and flat. It is known that mitogen-activated protein kinase kinase (MEK) inhibitors, such as U0216, can inhibit the activation of its downstream target EGR1 61,62, which we experimentally verified (Supplementary Fig. 4). Interestingly, U0216 also decreased TGF- $\beta$ R-II expression on the PS1018 surface.

We further observed a 2.5 fold increase of the TGF- $\beta$ inducible gene SCX after 8 hours, with maximum SCX levels after $24 \mathrm{~h}$ (3.5 fold change), which decreased to 2.8 fold after 48 hours. After $72 \mathrm{~h}$, no significant SCX elevation was detected (Fig. 3C). We also found that U0216 reduced topography-induced SCX upregulation. These findings demonstrate that microtopographies enhance SCX levels in MSCs, which could be guided by a general sensitization for TGF- $\beta$ signaling through SRF, EGR1), and TGF- $\beta$ R-II (Fig. 3D).

\section{Surface topography and TGF-62 synergistically induce TGF-6 target genes}

Given that SCX can be induced by both TGF- $\beta 2{ }^{26}$ and topography ${ }^{43}$, we investigated the combined effect of TGF- $\beta 2$ and micro-topographies on TGF- $\beta$ signaling. First, we measured SMAD2/3 phosphorylation (P-SMAD) as an immediate response to TGF- $\beta$ receptor signaling and mediator of TGF- $\beta$ target gene expression. $24 \mathrm{~h}$ time after cell seeding, the timepoint with maximum TGF- $\beta$ R-II expression, we exposed MSCs to TGF- $\beta 2$ and fixed the cells 30 minutes after the treatment (Fig. 4A). Quantification of nuclear P-SMAD levels demonstrated that TGF$\beta 2$ treatment resulted in a 1.2 fold increase in nuclear P-SMAD levels compared to cells cultured on flat (Fig. 4B). Of interest, we observed that TGF- $\beta 2$ treatment of MSCs grown on PS-1018 resulted in a 1.7-fold increase in nuclear P-SMAD levels compared to non-treated cells. Next, we isolated the RNA of MSCs cultured on flat or PS-1018, treated with and without TGF- $\beta 2$ between $4 \mathrm{~h}$ and seven days after cell seeding (Fig. 4C). The most striking and important observation we made is a synergistic induction of SCX expression at $24 \mathrm{~h}$. Whereas SCX levels on PS-1018 were 3.5 fold higher compared to a regular flat surface, TGF- $\beta 2$ stimulation alone resulted in a 14.5 fold increase in SCX levels compared to flat. Of interest, is that the combined exposure of MSCs to PS-1018 and TGF- $\beta 2$ induced SCX 39.9 fold. This synergy was already detected after 8 hours, although at lower levels, and was observed during the whole seven days culture period. It is interesting to note that SCX expression declines over time.

The synergistic response to TGF- $\beta 2$ on surface PS-1018 was not unique to MSCs. We induced a similar biological response in human dermal fibroblasts and C3H10T1/2 cells, a mouse mesenchymal-like cell line that is frequently utilized in differentiation studies (Fig. 4D). These findings demonstrate that the synergy between TGF- $\beta$ signaling and surface topography is reproducible in multiple TGF- $\beta 2$-response cell lines. Besides $S C X$, we also found a similar effect for other TGF- $\beta$ responsive genes. Expression of $a-S M A$, a differentiation marker of smooth muscle cells and myofibroblasts ${ }^{63}$, and the chondrogenic transcription factor $S_{0 X 9}{ }^{64}$ can be induced by TGF- 32 , and display a synergistic effect when combined with micro-topographies (Fig. 4E-F). These observations demonstrate that micro-topographies sensitize cells for TGF$\beta$ signaling. 
A
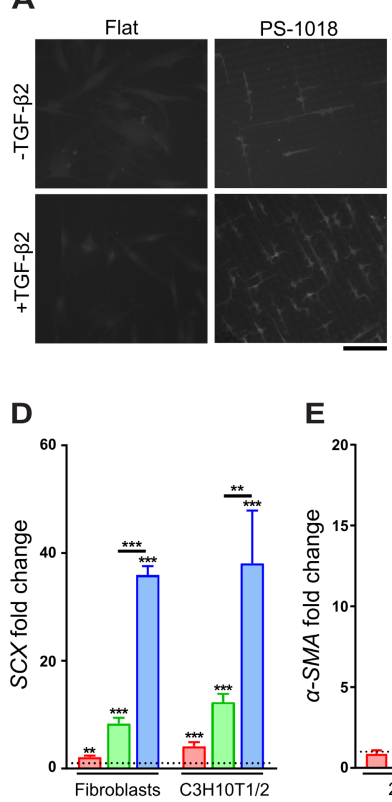

B

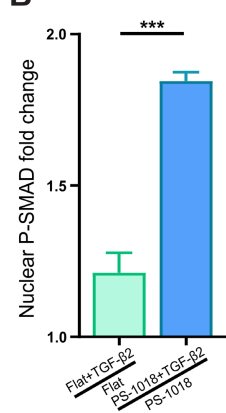

C



$\mathbf{F}$
E



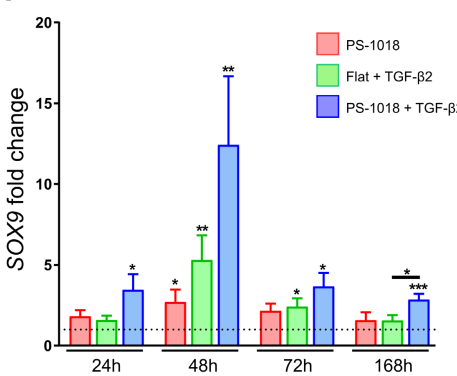

Figure 4: Micro-topographies positively modulate TGF- $\beta$ signaling. A-B) P-SMAD immunolabeling revealed elevated nuclear distribution in MSCs cultured on the PS-1018 compared to flat when treated with TGF- $\beta 2$ for 30 minutes. Scale bar represents $100 \mu \mathrm{m}$. C) TGF- $\beta$ treatment induces elevated SCX expression already $4 \mathrm{~h}$ after cell seeding, while PS-1018 induces an observable increase after $8 \mathrm{~h}$. Also at $8 \mathrm{~h}$, improved SCX levels are observed when combining micro-topographies with TGF- $\beta 2$. Peak SCX levels are reached at $24 \mathrm{~h}$ for all conditions. After $24 \mathrm{~h}$, overall $S C X$ levels drop. Micro-topographies however retain their synergistically effect when combined with TGF- $\beta 2$ at $48 \mathrm{~h}, 72 \mathrm{~h}$ and 7 days. D) Synergistic effect on SCX expression induced by the micro-topography can be reproduced with dermal fibroblasts and the mouse mesenchymal stem cell line C3H10T1/2. E) Improved a-SMA expression is observed when combining both PS-1018 and TGF- $\beta 2$ at $72 \mathrm{~h}$ and 7 days. F) Improved SOX9 expression is observed when combing PS-1018 and TGF- $\beta 2$ at $48 \mathrm{~h}$ and 7 days (* $\mathrm{P}<0.05$; ** $\mathrm{P}<0.01$; $* * * \mathrm{P}<0.001)$. Barplots represent the mean with error bars representing SEM.

\section{Rho/ROCK/SRF signaling is required for topography-induced TGF- $B$ sensitization}

We next set out to investigate the signaling events that occur between surface topographyinduced actin-mediated signaling and transcriptional activation of TGF- $\beta$ target genes, by investigating the synergistic effect in the presence of several small-molecule inhibitors of signal transduction. We confirmed the synergy in the presence of DMSO, the diluent of the inhibitors used in the rest of the study (Fig. 5A), and validated that SCX gene expression is indeed dependent on TGF- $\beta$ receptor activation, using its inhibitor SB431542 ${ }^{65}$ (Fig. 5B). Interestingly, this compound abolished SCX expression in MSCs on PS-1018 alone, which indicates that even without the addition of TGF- $\beta 2$, TGF- $\beta /$ SMAD signaling is occurring. This may hint at autoor paracrine signaling elicited by the MSCs, or TGF- $\beta$ originating from the serum media. 
Next, we studied the effect of the Rho-associated protein kinase (ROCK) inhibitor Y27632, because Rho proteins influence numerous biological responses, including cell shape and actin cytoskeletal rearrangement ${ }^{66}$ and are important for driving cell behavior of cells grown on physical cues. Y27632 did not affect the induction of SCX by TGF- $\beta 2$ on flat control surfaces but did abolish the synergistic effect on the PS-1018 surface (Fig. 5C). Very similar results were obtained with CCG-203971, a compound which inhibits SRF/Myocardin Related Transcription Factor A (MRTF) gene transcription ${ }^{67}$ (Fig. 5D) and blebbistatin (Fig. 5E), an inhibitor of nonmuscle myosin-II ${ }^{68}$ which prevents actin-myosin interaction resulting in subsequent disruption of actin dynamics ${ }^{69}$. Of interest, utilizing the MEK inhibitor U0216 did lower the level of SCX but did not abolish the synergistic effect (Fig. 5F), which indicates that the topography-induced mechanotransduction is not guided through MAPK signaling. These observations demonstrate

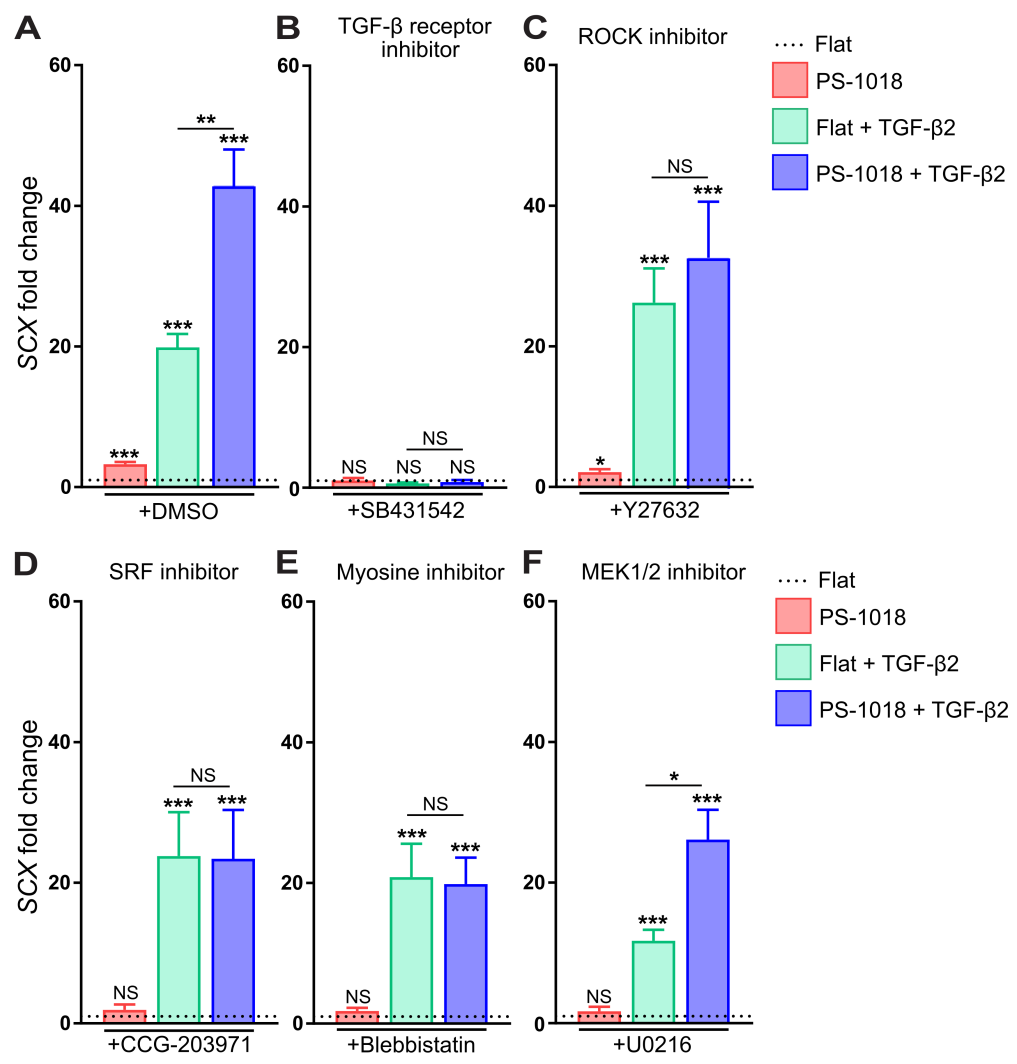

Figure 5: Pathway inhibitors reveal that micro-topographical induced $S C X$ expression requires Rho/ROCK/SRF signaling. A) DMSO does not affect SCX levels in each condition. B) Inhibitors against the TGF- $\beta$ receptor abolish SCX levels on all conditions including PS-1018, emphasizing the involvement of TGF- $\beta$ signaling for micro-topographical induction of SCX. C-E) The Rho/ROCK/SRF inhibitors Y27632, CCG-209371, and blebbistatin abolishes the topography-induced effect on SCX expression. F) The MAPK/MEK inhibitor U0216 reduces overall SCX expression, yet does not reduce the synergistic effect on SCX expression (* $\mathrm{P}<0.05$; *** $\mathrm{P}<0.001)$. Barplots represent the mean with error bars representing SEM. 
that actin dynamics and the Rho/ROCK/SRF signaling pathway is necessary for microtopographies to enhance the expression of $S C X$, but not for basal TGF- $\beta 2$ activity.

\section{PKC activators mimic topography-induced mechanotransduction}

Small molecules that inhibit actin-related signaling were able to block topographic induction of TGF- $\beta 2$ signaling, so vice versa, it may be possible to mimic topographic mechanotransduction with small molecules that mimic actin-related signaling. To find these molecules, we searched the Connectivity Map, a compendium of more than one million gene expression profiles induced by small molecules and genetic perturbations, which is used for determining similarities in gene expression profiles between these perturbations ${ }^{70}$. We previously described that topographyinduced TGF- $\beta 2$ signaling coincides with high levels of F-actin and concomitant SRF signaling after $1 \mathrm{~h}$, but reduced F-actin and genes related to Rho/ROCK/SRF signaling after $24 \mathrm{~h}$. We reasoned that topography mimicking small molecules should, therefore, recapitulate the actin dynamics observed on topographies. The Connectivity Map only contains gene expression data from later time points, and we therefore retrieved small molecules using the gene expression fingerprint of cells in which $\beta$-actin, $S R F$, and FOS genes were knocked down as bait for the search (Fig. 6A-C), because this best reflects the lower Rho/ROCK/SRF signaling axis after 24 hours. Of interest, perturbagen class "Protein Kinase C (PKC) activators" was present in the lists of $\beta$-actin (ACTB), SRF, and FOS and thus resembles gene signatures associated with these knockdowns. Furthermore, in the list of PKC activators, "MEK inhibitors" were present with a negative score, which could indicate a positive involvement for EGR1 (Fig. 6D). Also for tubulin, of which we see a decrease of multiple isoforms in the microarray data, we see an

A

\begin{tabular}{|c|c|}
\hline \multicolumn{2}{|c|}{ ACTB perturbagen class score } \\
\hline Score & Name \\
\hline 96.44 & BCL inhibitor \\
\hline 96.09 & HIF activator \\
\hline 95.04 & Ribonucleotide reductase inhibitor \\
\hline 94.94 & PKC activator \\
\hline
\end{tabular}

PKC perturbagen class score

A B C Score Name

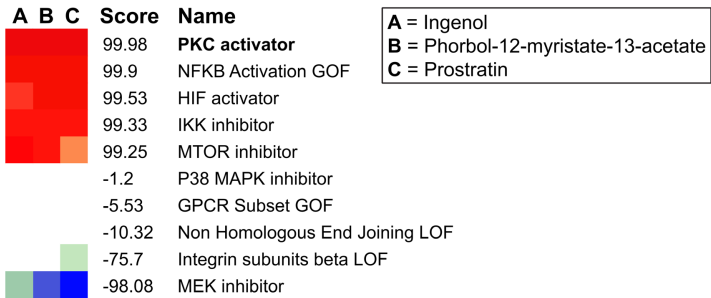

B

SRF perturbagen class score Score Name

97.90 Tubulin inhibitor

95.69 PKC activator

93.87 Wnt family LOF

93.49 Kruppel like transcription factors LOF
C

FOS perturbagen class score

Score Name

94.61 NFKB activation GOF

92.74 PKA inhibitor

88.94 Glycogen synthase kinase inhibitor

88.48 PKC activator

E

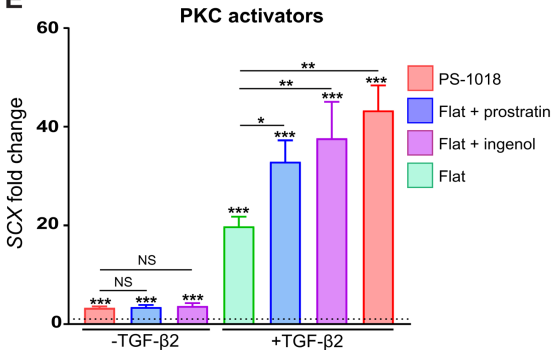

Figure 6: The connectivity map associates the gene signature of PKC activators with topographyinduced mechanotransduction. A) $A C T B$ knockdown gene signature corresponds with a PKC activator score of 94.94. B) $S R F$ knockdown gene signature corresponds with a PKC activator score of 95.69. C) FOS knockdown gene signature corresponds with a PKC activator score of 88.48. D) PKC perturbagen class gene signature corresponds with a MEK inhibitor score of -98.08. E) Through qPCR, we found that the PKC activators prostratin and ingenol reproduce the micro-topographical effect on $S C X$ expression, both with- and without the addition of TGF- $\beta 2\left(* \mathrm{P}<0.05\right.$; ${ }^{* *} \mathrm{P}<0.01$; $\left.* * * \mathrm{P}<0.001\right)$. Barplots represent the mean with error bars representing SEM. 
association with PKC activators (Supplementary Fig. 5), suggesting that treatment of cells with PKC activators leads to similar gene expression profiles as when $A C T B, S R F$, and FOS are knocked-down.

PKC activators can induce actin reorganization ${ }^{71}$, which eventually leads to a decrease in actin stress fibers ${ }^{72}$. Furthermore, they can activate FOS ${ }^{73}$ and EGR $1^{74}$. This makes this perturbagen class an interesting candidate for molecules that can mimic topography-induced TGF- $\beta$ sensitization. We want to mention that in the $\beta$-actin knockdown gene fingerprint list, we found gene signature resemblances with other perturbagens such as cytochalasin- $\mathrm{B}$, a microtubule inhibitor, and cytochalasin-D, an actin polymerization inhibitor (Supplementary Fig. 6). However, as shown with the actin polymerization inhibitor blebbistatin, this compound fails to recapitulate the synergistic effect with TGF- $\beta 2$, since unlike PKC activators, no activation of early response genes is achieved. Also of interest is that an EGR1 knockdown corresponds strongly with the gene signature of a TGF- $\beta$ R-II knockdown, further emphasizing the relationship between these genes (Supplementary Fig. 7).

From the PKC activator component list (Supplementary Fig. 8), ingenol and prostratin were added to MSCs with and without TGF- 32 , and SCX levels were measured after $24 \mathrm{~h}$. Without TGF- $\beta 2$, ingenol and prostratin induced similar SCX levels as the PS-1018 surface (Fig. 6E). In the presence of TGF- $\beta 2$, prostratin induced a 36.2 fold SCX expression compared to a regular flat surface without TGF- $\beta 2$. Ingenol-3 induced a similar response, with slightly higher SCX levels (39.1 fold change) than prostratin. No significant differences between the topography and PKC activators were observed when adding TGF- $\beta 2$. These findings demonstrate that PKC activators strikingly mimic the effect of the micro-topography in inducing SCX levels.

In previous work, we found that tenocytes rapidly lose their phenotypic characteristics in cell culture $^{43}$. Cells transform from a spindle-shape towards a rounded morphology, which coincides with the formation of F-actin stress fibers. We were therefore interested in investigating if PKC activators could improve phenotypic characteristics in tenocyte culture. Therefore, we treated passage one tenocytes with- and without the PKC activators and TGF- $\beta 2$ and found more profound spindle-shaped characteristics in confluent conditions when cells were treated with ingenol, both without and with TGF- $\beta 2$ (Fig. 7A). To further investigate their morphological characteristics, we fixed cells $72 \mathrm{~h}$ after adding the compounds and stained for F-actin, and SCX. Ingenol caused an apparent reduction in F-actin stress fibers and a decrease in F-actin intensity levels (Fig. 7B). Of interest is that TGF- $\beta 2$ induces a more spread out morphology, which coincided with higher F-actin levels. This observation was also abolished by ingenol. We found that TGF- $\beta 2$ was able to increase SCX levels, which could be further amplified by the ingenol treatment (Fig. 7C). These novel findings demonstrate that PKC activators replace the synergistic effects of the micro-topography both in the context of TGF- $\beta$ induced MSC differentiation and phenotypic maintenance of tenocytes. 
A

DMSO


B



Ingenol


TGF- $\beta 2$
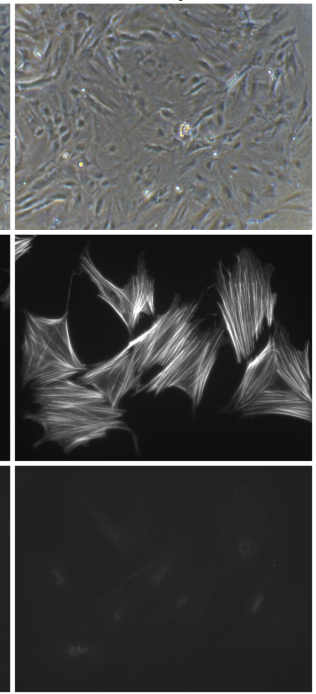

Ingenol+TGF- $\beta 2$
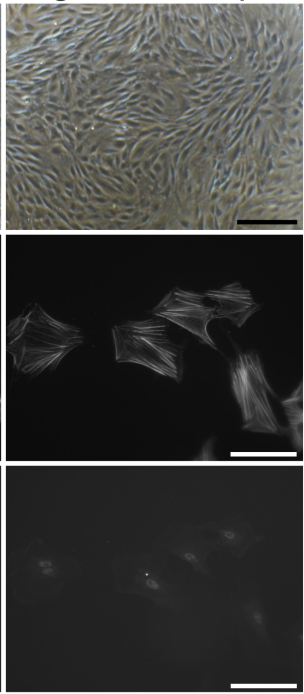

C

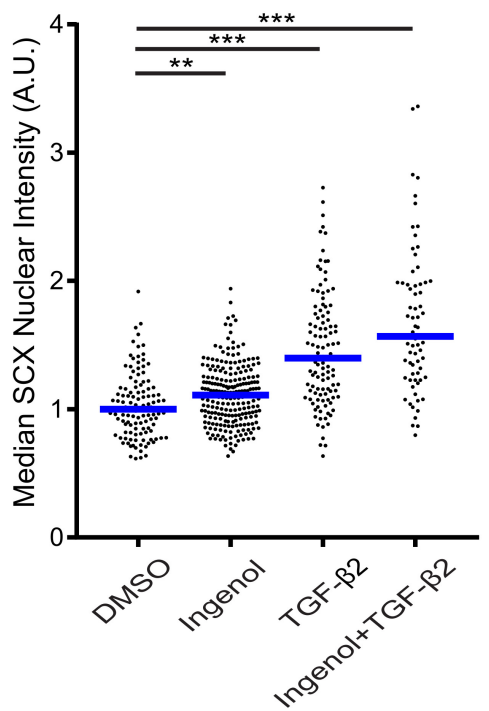

Figure 7: PKC activators reduce F-actin and elevate SCX in tenocytes. A) Ingenol-3 induces elongated cell characteristics in confluent conditions. Furthermore, reduced F-actin levels in tenocytes both with- and without a TGF- $\beta 2$ treatment are observed when treated with ingenol. Similar was with MSCs, SCX levels increase by treating tenocytes with a PKC activator. Scale bar represent $100 \mu \mathrm{m}$. B) F-actin immunolabeling quantification reveals significant lower levels when tenocytes are treated with ingenol, both with- and without TGF- $\beta 2$. C) SCX immunolabeling quantification reveals significant increased levels when cells are treated with ingenol and TGF- $\beta 2$, with a most optimal effect when combining both perturbations $(* * * \mathrm{P}<0.001)$. Dotplots represent intensity levels of individual nuclei. Blue line indicates median. 


\section{Discussion}

We present evidence that TGF- $\beta$ signaling is positively modulated through mechano-induced topographical cues. This work emphasizes the importance of recapitulating the crosstalk typically seen in vivo between mechanical and soluble cues in an in vitro culture setting. In addition, the results of this work put other research from the tissue-engineering field in a new perspective. BMP-2 is a growth factor that improves bone formation in a clinical setting, but only at unphysiologically high levels of the protein ${ }^{75}$. We hypothesize that BMP-2 is presented in the wrong mechanical context, and mechanical fine-tuning can lead towards greater efficacy. Research combining BMP-2 with mechanical loading indeed seems to indicate this ${ }^{76}$. Similarly, VEGF promotes angiogenesis, yet it also leads to vascular disruption ${ }^{77}$. Here it is plausible that the underlying mechanisms will be better controlled by implementing alternative stimulation as well. It is therefore interesting to reconsider growth factor-mediated cell differentiation and cell growth in the context of biomechanical conditions.

In an in vitro context, other studies involving geometrical-induced modulation of tenogenic gene expression do not consider the potential synergistic effects of growth factor signaling in their experimental setup ${ }^{43,78-82}$. An exception is a study documenting improved SCX expression in a dermal fibroblast culture on micro-grooves combined with TGF- $\beta 1{ }^{83}$. Besides microtopographies, other physical cues such as scaffolds also enhance tenogenic characteristics of MSCs and progenitor cells 84,85 , making this an interesting material design parameter for exploring if it might modulate TGF- $\beta$ signaling. In this context, matrix stiffness can influence SCX expression ${ }^{86}$, another physical environmental factor known for modulating TGF- $\beta$ signaling ${ }^{87-89}$. These examples highlight the potential of future research involving biomaterials that investigate interactions with TGF- $\beta$ signaling and other signaling cascades elicited by soluble cues.

In this study, we found mechanobiological signaling similarities with other experimental setups. For example, increased levels of EGR1 during the cell adaptation phase are also found early upon cell stretching together with increased levels of FOS ${ }^{33}$. EGR1 plays a crucial role in tenocyte mechanical signaling and can influence TGF- 3 R-II ${ }^{53}$, SCX, and other tenogenic markers ${ }^{50-52}$. It is therefore not surprising that mechanical stimulation through stretching is studied extensively for inducing MSC differentiation ${ }^{90,91}$, and tenogenic matrix deposition ${ }^{92}$. Furthermore, a clear link exists between mechanical forces and TGF- $\beta$ /SMAD activation in numerous cell types ${ }^{93-95}$. SRF activity plays a central role in mediating actin dynamics ${ }^{14,96}$, which is clearly altered and dynamically modulated by physical cues in this study. A plausible scenario is that SRF binds to serum response elements (SRE) in its promotor and those of FOS ${ }^{48}$ and EGR1 ${ }^{97}$, triggering the biological events we observe. This concept is further emphasized through the use of PKC activators, known to induce SRE activation ${ }^{73}$, and thereby mimicking the effect of the topography. Although physical cues can regulate Rho/ROCK signaling ${ }^{19}$, evidence points towards SRF as a subsequent and essential mediator in the observed biomechanical signaling events 22,24,25. Also, experimental readouts that involve mechanical 
stretching can be blocked by inhibiting the Rho/ROCK/SRF pathway ${ }^{98,99}$. Related to this, the concept that SRF is involved in TGF- $\beta$ signal transduction is not novel. Evidence exists that force-induced activation of $\alpha$-SMA and subsequent fibrosis is blocked by inhibiting the Rho/ROCK pathway and subsequent SRF activity ${ }^{17,100}$.

This research provides novel insights into how physical cues transmit mechanobiological signaling. Especially the dynamical nature of the signaling cascades elicited by the microtopographies is fascinating. Parts of the mechanisms behind the phenomena we observe are however still unknown. For example, we do not rule out that FOS plays a role in TGF- $\beta$ sensitization due to a binding affinity against SMAD2/3 54. Also, despite the apparent requirement of SRF for inducing TGF- $\beta$ sensitivity, other transcription factors might play a role in activating SRE's of EGR1 and FOS in conjunction with SRF ${ }^{101}$. More insights will need to be gained by investigating how much pathway overlap there is between physical cues, PKC activation, and mechanical stretching. The observation that PKC activators mimic topographyinduced biomechanical stimulation is a fascinating concept since it extends the translational perspectives of our experiments. Small molecules inducing the same synergistic effect as microtopographies could be introduced into a clinical context where mechanical loading events typically support tissue repair. For tendon tissue-engineering, this implies the potential to utilize PKC activators for stimulating a growth factor-mediated healing response, which might improve current clinical treatments ${ }^{102}$.

\section{Conclusion}

We demonstrate that micro-topographies influence TGF- $\beta$ signaling in MSCs, leading to increased expression of the differentiation markers SCX, SOX9, and a-SMA. We connect the origin of this mechanobiological signaling with actin remodeling elicited by physical cues, which coincides with subsequent activation of early response genes associated with an upregulation of TGF- $\beta$ R-II. Through extensive pathway analysis, we identify small molecule compounds that mimic the effect of the micro-topography. The results of this study can lead to improved protocols for the differentiation of MSCs or phenotypic maintenance of primary cells involving TGF- $\beta$ signaling. Furthermore, the identification of small molecules that mimic mechanobiological stimulation might be applied in a clinical setting for replacing mechanical stimulation in conjunction with soluble cues.

\section{Materials and Methods}

\section{Surface fabrication}

A detailed description of the surface fabrication procedures is found elsewhere ${ }^{103}$. In short, the inverse pattern of the topographies was etched into a silicon wafer by directional reactive ion etching (DRIE). To facilitate demoulding procedures, the wafer was coated with a layer of Trichloro(1H,1H,2H,2H-perfluorooctyl)silane (FOTS, Sigma-Aldrich). Polydimethylsiloxane (PDMS; Down Corning) was cured on the silicon wafer to generate a positive mould and was 
subsequently used as a template to create a second negative mould in Ormostamp polymer (micro resist technology Gmbh), which serves as the mold for hot embossing the polystyrene (PS) films (Goodfellow). The hot embossing procedure was carried out at $140{ }^{\circ} \mathrm{C}$ for $5 \mathrm{~min}$, and a pressure of $10 \mathrm{Bar}$, with a demoulding temperature of $90^{\circ} \mathrm{C}$. Before cell culture, the PS topographies were treated with oxygen plasma to improve cell adhesion for $30 \mathrm{~s}$ at $0.8 \mathrm{mbar}, 50$ sccm $\mathrm{O}_{2}$, and $100 \mathrm{~W}$. Quality of the fabricated imprints was assessed using a Keyence VKH1XM-131 profilometer.

\section{Cell culture}

Adipose-derived human mesenchymal stem cells (AD-hMSCs) and dermal fibroblasts (DF) used in this study were purchased from Lonza. AD-hMSCs and DF were isolated from a 42-year-old, and 27-year-old female respectively. C3H10T1/2 cells were purchased at ATCC. All methods were performed in accordance with the relevant ethical guidelines and regulations of the University of Maastricht. Basic medium for AD-hMSCs and DF consists of MEM Alpha GlutaMAX, no nucleosides (Gibco). For the culture of C3H10T1/2 cells, DMEM low glucose (Merck) was used as basic media. Basic media was supplemented with $10 \% \mathrm{v} / \mathrm{v}$ fetal bovine serum (FBS), $\quad 0.2 \mathrm{mM}$ ascorbic-acid-2-phosphate (ASAP), and $10 \mathrm{U} / \mathrm{mL}$ Penicillin/Streptomycin. Cells were grown at $37{ }^{\circ} \mathrm{C}$ in a humid atmosphere at $5 \% \mathrm{CO}_{2}$. For experimental purposes, cells at passage 3-4 were seeded at a density of $5000-10000$ cells $/ \mathrm{cm}^{2}$ on flat and the topographical surface. Human TGF- $\beta 2$ (Peprotech), and mouse TGF- $\beta 2$ (R\&D Systems) were included in the media during cell seeding at a final concentration of $20 \mathrm{ng} / \mathrm{ml}$. Pathway inhibitors blebbistatin, Y27632, CCG-203971, U2016, and SB431542 were purchased from Sigma-Aldrich and included in the media at a final concentration of $10 \mu \mathrm{M}$. For inhibitor studies, inhibitors were added to the cell media $1 \mathrm{~h}$ prior and during cell seeding. PKC activators Ingenol, and Prostratin were purchased from Sigma-Aldrich, and added to the medium at a final concentration of $10 \mu \mathrm{M}$ during cell seeding or media replacement.

\section{Microarray study and pathways analysis}

Bone marrow-derived human MSCs were seeded on topography PS-281 for 24 hours in basic medium at a density of 15,000 cells/cm2 in 24 well plates in three replicas. Total RNA was isolated using the Nucleospin RNA isolation kit (Macherey-Nagel). Then, from $100 \mathrm{ng}$ of RNA, cRNA was synthesized using the Illumina TotalPrep RNA amplification kit. Both RNA and cRNA quality was verified on a Bioanalyzer 2100 (Agilent). Microarrays were performed using Illumina HT-12 v4 expression Beadchips. $750 \mathrm{ng}$ of cRNA was hybridized on the array overnight, after which the array was washed and blocked. Then, through the addition of streptavidin Cy-3, a fluorescent signal was developed. Arrays were scanned on an Illumina Beadarray reader, after which raw intensity values were background corrected in BeadStudio (Illumina). Further data processing and statistical testing were performed using the online portal arrayanalysis.org. The probe-level raw intensity values were quantile normalized and transformed using variance stabilization (VSN). A detection threshold of 0.01 was used for reducing the number of false positives. A linear modelling approach with empirical Bayesian methods, as 
implemented in Limma package, was applied for differential expression analysis of the resulting probe-level expression values.

To construct a gene network of the DEGs, we applied an online STRING analysis (https://string-db.org/). Only DEG with a fold change higher than 1.5 and an adjusted p-value lower than 0.05 were included in the list.

For identifying small molecules that mimic topography-induced pathways, we searched for relevant genes affected by the micro-topography in the connectivity map (https://clue.io/).

\section{Phospho proteomics study}

In-liquid digestion

A total of $60 \mu \mathrm{g}$ protein in $50 \mu \mathrm{l} 50 \mathrm{mM}$ ammonium bicarbonate (ABC) with $5 \mathrm{M}$ urea was used. $5 \mu \mathrm{L}$ of dithiothreitol (DTT) $(20 \mathrm{mM}$ final) was added and incubated at room temperature for $45 \mathrm{~min}$. The proteins were alkylated by adding $6 \mu \mathrm{L}$ of IAA solution ( $40 \mathrm{mM}$ final). The reaction was taken place at room temperature for $45 \mathrm{~min}$ in the darkness. The alkylation was stopped by adding $10 \mu \mathrm{L}$ of DTT solution (to consume any unreacted IAA) and incubated at room temperature for $45 \mathrm{~min}$. For the protease digestion, $2 \mu \mathrm{g}$ trypsin/lys $\mathrm{C}$ was added to the protein and incubated at $37^{\circ} \mathrm{C}$ for $2 \mathrm{~h} .200 \mu \mathrm{l}$ of $50 \mathrm{mM} \mathrm{ABC}$ was added to dilute the urea concentration and further incubate at $37^{\circ} \mathrm{C}$ for $18 \mathrm{~h}$. The digestion mix was centrifuge at $2 \times 10^{3} \mathrm{~g}$ for $5 \mathrm{~min}$ and the supernatant collected.

Phospho-peptides were enriched by using TiO2 spin columns according the manufacturers protocol (Thermo Scientific). Samples were subsequently labelled with TMT isobaric mass tagging labelling reagent (10-plex; Thermo Scientific) according to the manufacturer's protocol. In short, $60 \mu \mathrm{g}$ of protein for each sample was used. The TMT labelling reagents were dissolved in $41 \mu \mathrm{l}$ acetonitrile per vial. The reduced and alkylated samples and control were added to the TMT reagent vials. The reaction was incubated for $1 \mathrm{~h}$ at room temperature and quenched for $15 \mathrm{~min}$ by adding $8 \mu \mathrm{l}$ of $5 \%$ hydroxylamine. Equal amounts of the samples and control were combined in a new vial and analysed by liquid chromatography-tandem mass spectrometry (LC$\mathrm{MS} / \mathrm{MS})$.

\section{Protein identification using LC-MS/MS}

A nanoflow HPLC instrument (Dionex ultimate 3000) was coupled on-line to a Q Exactive (Thermo Scientific) with a nano-electrospray Flex ion source (Proxeon). The final concentration of the TMT labeled digest/peptide mixture was $0.33 \mu \mathrm{g} / \mu \mathrm{l}$ and $5 \mu \mathrm{l}$ of this mixture was loaded onto a C18-reversed phase column (Thermo Scientific, Acclaim PepMap C18 column, 75- $\mu \mathrm{m}$ inner diameter x $15 \mathrm{~cm}, 2-\mu \mathrm{m}$ particle size). The peptides were separated with a 90 min linear gradient of $4-68 \%$ buffer B (80\% acetonitrile and $0.08 \%$ formic acid) at a flow rate of 300 $\mathrm{nL} / \mathrm{min}$.

MS data was acquired using a data-dependent top-10 method, dynamically choosing the most abundant precursor ions from the survey scan $(280-1400 \mathrm{~m} / \mathrm{z})$ in positive mode. Survey scans 
were acquired at a resolution of $70 \times 10^{3}$ and a maximum injection time of $120 \mathrm{~ms}$. Dynamic exclusion duration was $30 \mathrm{~s}$. Isolation of precursors was performed with a $1.8 \mathrm{~m} / \mathrm{z}$ window and a maximum injection time of $200 \mathrm{~ms}$. Resolution for HCD spectra was set to 30,000 and the Normalized Collision Energy was $32 \mathrm{eV}$. The under-fill ratio was defined as $1.0 \%$. The instrument was run with peptide recognition mode enabled, but exclusion of singly charged and charge states of more than five.

Database search

The MS data were searched using Proteome Discoverer 2.2 Sequest HT search engine (Thermo Scientific), against the UniProt human database. The false discovery rate (FDR) was set to 0.01 for proteins and peptides, which had to have a minimum length of six amino acids. The precursor mass tolerance was set at 10 parts per million (ppm) and the fragment tolerance at $0.02 \mathrm{Da}$. One miss-cleavage was tolerated, Phosphorylation of serienes, tyrosines and threonines as well as oxidation of methionine were set as a dynamic modification. Carbamidomethylation of cysteines, Tandem mass tag (TMT) reagent adducts (+229.162932 Da) on lysine and peptide amino termini were set as fixed modifications.

\section{Immunocytochemistry}

After cell culture, the cells were washed with phosphate buffered saline (PBS; Merck) and fixed with $4 \%(\mathrm{w} / \mathrm{v})$ paraformaldehyde (Sigma-Aldrich) for $5 \mathrm{~min}$ at $37^{\circ} \mathrm{C}$. After a washing step, cells were permeabilized with $0.01 \%$ (v/v) Triton X-100 (Acros Organics) and blocked with goat serum (1:100; Sigma-Aldrich) in PBT (PBS + 0.02\% Triton-X-100, 0.5\% BSA) for $1 \mathrm{~h}$. Afterwards, cells were incubated with the primary antibody in PBT for $1 \mathrm{~h}$. After a washing step, cells were incubated with a secondary antibody targeting the primary antibody protein of interest and conjugated to an Alexa Fluor (1:500; ThermoFisher) in PBT. After washing, the nucleus was counterstained with Hoechst 33258 (1:1000; Sigma-Aldrich) for $10 \mathrm{~min}$. After a subsequent washing step, surfaces were mounted on glass cover slides with mounting media (Dako). All washing steps were performed in triplicate with PBT. Primary antibodies used in this study are: anti-SCX antibody (1:200; Abcam; ab58655), anti-EGR1 antibody (1:200; ThermoFisher; T.126.1), anti- Phospho-Smad2/3 (1:200; Cell Signaling Technologies; 8828S), and anti-SRF antibody (1:200; Santa Cruz; sc-335).

\section{Image analysis}

Fixed samples were inverted and fluorescent images were acquired through the glass coverslip using a fully automated Nikon Eclipse Ti-U microscope in combination with an Andor Zyla 5.5 4MP camera. Fluorescent images were analyzed through CellProfiler 3.1.8 ${ }^{104}$ applying custommade pipelines. All images were cropped in order to remove out-of-focus objects. Objects touching the border of the subsequent images were filtered out of the dataset. After illumination corrections, morphology of the nucleus was captured by the Otsu adaptive thresholding method applied on the Hoechst 33258 image channel. Subsequently, cell morphology was determined by applying propagation and Otsu adaptive thresholding on the Phalloidin image channel. 
Missegmentation artifacts were removed by applying an arbitrary threshold on nuclei and cell size. After background correction, intensity values of the target of interest were calculated either inside the nuclear or cell area. The image software Fiji was used for image visualization ${ }^{105}$.

\section{Quantitative polymerase chain reaction ( $q P C R)$}

After cell lysis by Trizol (Thermo Fisher), total RNA was isolated using the RNeasy Mini Kit (Qiagen) according to the manufacturer's protocol. Reverse transcription was carried out using an iScript ${ }^{\mathrm{TM}} \mathrm{cDNA}$ synthesis kit (Bio-Rad). Quantitative PCR was performed using the $\mathrm{iQ}^{\mathrm{TM}}$ SYBR ${ }^{\circ}$ Green Supermix (Bio-Rad) in a CFX96 Touch Real-Time PCR Detection System (Bio$\mathrm{Rad})$. The software qBase+ allowed determining relative expression using the $\Delta \Delta \mathrm{Ct}$ method. The geometric mean of the reference genes Glyceraldehyde 3-phosphate dehydrogenase $(G A P D H)$ and TATA-Box Binding Protein (TBP) was applied for normalization. Primer sequences are listed in Supplementary Table 3.

\section{Statistical analysis}

Statistical analysis was performed with GraphPad Prism (GraphPad Prism Software Incl. San Diego, USA). For image analysis where more than two experimental conditions are considered, a two-way ANOVA was applied for determining statistical significance between conditions. Each data point represents a measurement from an individual cell. Image experiments were replicated with similar results. For image analysis with two experimental conditions, such as is the case for the P-SMAD data, a two-sided T-test was applied. For qPCR data, two-sided T-test statistics was applied for determining if the fold change of a condition is significant different than the flat control. One-way ANOVA was applied for determining statistical significance of the fold changes between conditions. qPCR data is based on at least three independent experiments, with each data points representing an independent experiment. Microarray P-values were corrected for multiple testing using the Benjamini and Hochberg method. Genes with a corrected p-value below 0.05 were considered differentially expressed.

\section{MSC osteogenic and adipogenic differentiation}

To assess if the AD-MSCs were multipotent during the experiments, we investigated their potential for differentiation towards the osteogenic and adipogenic lineage. Differentiation of AD-hMSCs towards the osteogenic lineage was achieved by seeding AD-hMSCs at a density of $5 \times 10^{3}$ cells $/ \mathrm{cm} 2$. After $24 \mathrm{~h}$, medium was changed with either a control or mineralization medium. The mineralization media is basic media supplemented with $10 \% \mathrm{v} / \mathrm{v}$ fetal bovine serum (FBS), $10 \mathrm{U} / \mathrm{mL}$ Penicillin/Streptomycin, with $10 \mathrm{nM}$ dexamethasone (Sigma) and 10 $\mathrm{mM} \beta$-glycerol phosphate (Sigma), while control medium includes the same components except dexamethasone. Media was refreshed every 2-3 days and after 21 days, cells were fixed overnight at $4{ }^{\circ} \mathrm{C}$ with $4 \%$ formaldehyde (VWR) in PBS. Afterwards, osteogenesis was assessed through staining mineralized deposits with a $2 \%$ Alizarin Red solution $(\mathrm{pH}=4.2)$ for 2 min. Excess staining was washed off with demineralized water (Supplementary Fig. 9A). 
Differentiation of AD-hMSCs towards the adipogenic lineage was achieved by seeding ADhMSCs at a density of $15 \times 10^{3}$ cells $/ \mathrm{cm} 2$. After $24 \mathrm{~h}$, medium was replaced with either a control or adipogenic medium. The adipogenic media consist of basic media supplemented with $10 \%$ $\mathrm{v} / \mathrm{v}$ fetal bovine serum (FBS), $10 \mathrm{U} / \mathrm{mL}$ Penicillin/Streptomycin, $0.5 \mathrm{mM}$ 3-isobutyl-1methylxanthine (Sigma), $0.2 \mathrm{mM}$ indomethacin (Sigma), $10 \mu \mathrm{g} / \mathrm{mL}$ Insulin (Sigma), and $1 \mu \mathrm{M}$ dexamethasone (Sigma). Control medium consisted solely of basic media with $10 \% \mathrm{v} / \mathrm{v}$ fetal bovine serum (FBS) and $10 \mathrm{U} / \mathrm{mL}$ Penicillin/Streptomycin. Media was refreshed every 2-3 days and after 21 days, cells were fixed overnight at $4{ }^{\circ} \mathrm{C}$ with $3.7 \%$ formaldehyde (VWR), $0.01 \mathrm{~g} / \mathrm{ml}$ $\mathrm{CaCl}_{2} \cdot 2 \mathrm{H}_{2} \mathrm{O}$ (Merck) in PBS. Afterwards, adipogenesis was assessed by rinsing the fixation solution with demineralized water, and subsequently incubating the substrates in a $60 \%(\mathrm{v} / \mathrm{v}) 2$ propanol (VWR) for $5 \mathrm{~min}$. Fat droplets were stained through a freshly filtered solution of $0.3 \%$ (w/v) Oil Red O dissolved in 60\% (v/v) 2-propanol (VWR). Afterwards, the substrates were washed in triplicate with demineralized water (Supplementary Fig. 9B).

\section{Acknowledgments}

SV, NR, FH, DH, AD, AC, and JdB acknowledge the financial support of the Dutch province of Limburg. SV and AD are supported by the European Union's Horizon 2020 Programme (H2020-MSCA-ITN-2015; Grant agreement 676338). PtD is supported by the Cancer Genomics Center Netherlands (CGC.NL). AC gratefully acknowledges her VENI grant (number 15057) from the Dutch Science Foundation (NWO). We thank Freek Bouwman, Edwin Mariman, and Midory Thorikay for expert technical assistance.

\section{Competing Interests}

The authors declare no competing interests.

\section{Data Availability Statement}

The raw/processed data required to reproduce these findings are available to download from DataverseNL (https:// dataverse.nl/privateurl.xhtml?token=f60fcaf3-a828-44db-821aa6ad3124ca6d).

\section{References}

1. Mammoto, T. \& Ingber, D. E. Mechanical control of tissue and organ development. Development 137, 1407-1420 (2010).

2. Goldspink, D. F. et al. Muscle growth in response to mechanical stimuli. Am. J. Physiol. Endocrinol. Metab. 268, 288-297 (1995).

3. Rosager, S., Aagaard, P., Neergaard, K., Kjaer, M. \& Magnusson, S. P. Load-displacement properties of the human triceps surae aponeurosis and tendon in runners and non-runners. Scand. J. Med. Sci. Sport. 12, 90-98 (2002).

4. Potter, R. M. et al. Impact of TGF- $\beta$ inhibition during acute exercise on achilles tendon extracellular matrix. Am. J. Physiol. - Regul. Integr. Comp. Physiol. 312, R157-R164 (2017).

5. Wipff, P. J., Rifkin, D. B., Meister, J. J. \& Hinz, B. Myofibroblast contraction activates latent TGF- $\beta 1$ from the extracellular matrix. J. Cell Biol. 179, 1311-1323 (2007). 
6. Etoc, F. et al. A Balance between Secreted Inhibitors and Edge Sensing Controls Gastruloid SelfOrganization. Dev. Cell 39, 302-315 (2016).

7. Eyckmans, J., Boudou, T., Yu, X. \& Chen, C. S. A Hitchhiker's Guide to Mechanobiology. Dev. Cell 21, 35-47 (2011).

8. Benham-pyle, B. W., Pruitt, B. L. \& Nelson, W. J. Mechanical strain induces E-cadherin dependent Yap1 and $\beta$-catenin activation to drive cell cycle entry. Science (80-. ). 348, 1024-1027 (2015).

9. Aragona, M. et al. A mechanical checkpoint controls multicellular growth through YAP/TAZ regulation by actin-processing factors. Cell 154, 1047-1059 (2013).

10. Maitre, J. L. et al. Asymmetric division of contractile domains couples cell positioning and fate specification. Nature 536, 344-348 (2016).

11. Elbediwy, A. et al. Integrin signalling regulates YAP and TAZ to control skin homeostasis. Dev. 143, 1674-1687 (2016).

12. Hong, J. et al. TAZ , a Transcriptional Modulator of Mesenchymal Stem Cell Differentiation. Science (80-. ). 309, 1074-1078 (2005).

13. Lee, K.-S. et al. Runx2 Is a Common Target of Transforming Growth Factor beta 1 and Bone Morphogenetic Protein 2, and Cooperation between Runx2 and Smad5 Induces Osteoblast-Specific Gene Expression in the Pluripotent Mesenchymal Precursor Cell Line C2C12. Mol. Cell. Biol. 20, 8783-8792 (2000).

14. Sotiropoulos, A., Gineitis, D., Copeland, J. W. \& Treisman, R. Signal-regulated activation of serum response factor is mediated by changes in actin dynamics. Cell 98, 159-169 (1999).

15. Miano, J. M., Long, X. \& Fujiwara, K. Serum response factor: Master regulator of the actin cytoskeleton and contractile apparatus. Am. J. Physiol. - Cell Physiol. 292, 70-81 (2007).

16. Li, S., Wang, D., Wang, Z., Richardson, J. A. \& Olson, E. N. The serum response factor coactivator myocardin is required for vascular smooth muscle development. PNAS 100, 9366-9370 (2003). 17. Zhao, X. H. et al. Force activates smooth muscle $\alpha$-actin promoter activity through the Rho signaling pathway. J. Cell Sci. 120, 1801-1809 (2007).

18. Hu, B., Wu, Z. \& Phan, S. H. Smad3 mediates transforming growth factor- $\beta$-induced $\alpha$-smooth muscle actin expression. Am. J. Respir. Cell Mol. Biol. 29, 397-404 (2003).

19. McBeath, R., Pirone, D. M., Nelson, C. M., Bhadriraju, K. \& Chen, C. S. Cell shape, cytoskeletal tension, and RhoA regulate stem cell lineage commitment. Dev. Cell 6, 483-495 (2004).

20. Kilian, K. A., Bugarija, B., Lahn, B. T. \& Mrksich, M. Geometric cues for directing the differentiation of mesenchymal stem cells. Proc. Natl. Acad. Sci. 107, 4872-4877 (2010).

21. Abagnale, G. et al. Surface topography enhances differentiation of mesenchymal stem cells towards osteogenic and adipogenic lineages. Biomaterials 61, 316-326 (2015).

22. Mitra, A. et al. Cell geometry dictates TNF $\alpha$-induced genome response. Proc. Natl. Acad. Sci. 114, E3882-E3891 (2017).

23. Wang, Y. et al. Bone Morphogenetic Protein-2-Induced Signaling and Osteogenesis Is Regulated by Cell Shape, RhoA/ROCK, and Cytoskeletal Tension. Stem Cells Dev. 21, 1176-1186 (2012).

24. Connelly, J. T. et al. Actin and serum response factor transduce physical cues from the microenvironment to regulate epidermal stem cell fate decisions. Nat. Cell Biol. 12, 711-718 (2010).

25. Jain, N. \& Vogel, V. Spatial confinement downsizes the inflammatory response of macrophages. Nat. Mater. (2018). doi:10.1038/s41563-018-0190-6

26. Havis, E. et al. Transcriptomic analysis of mouse limb tendon cells during development. Development 141, 3683-3696 (2014).

27. Greber, B., Lehrach, H. \& Adjaye, J. Fibroblast growth factor 2 modulates transforming growth factor beta signaling in mouse embryonic fibroblasts and human ESCs (hESCs) to support hESC selfrenewal. Stem Cells 25, 455-64 (2007).

28. Iwasaki, H., Eguchi, S., Ueno, H., Marumo, F. \& Hirata, Y. Mechanical stretch stimulates growth of vascular smooth muscle cells via epidermal growth factor receptor. Am. J. Physiol. Heart Circ. Physiol. 278, H521-9 (2000).

29. Tanabe, Y. et al. Mechanical stretch augments PDGF receptor $\beta$ expression and protein tyrosine phosphorylation in pulmonary artery tissue and smooth muscle cells. Mol. Cell. Biochem. 215, 103-113 (2000). 
30. Spassova, M. A., Gill, D. L., Soboloff, J., Hewavitharana, T. \& Xu, W. A common mechanism underlies stretch activation and receptor activation of TRPC6 channels. Proc. Natl. Acad. Sci. 103, 1658616591 (2006).

31. Singhvi, R. et al. Engineering Cell Shape and Function. Science (80-. ). 264, 696-698 (1994).

32. Unadkat, $\mathrm{H}$. V et al. An algorithm-based topographical biomaterials library to instruct cell fate. Proc. Natl. Acad. Sci. U. S. A. 108, 16565-70 (2011).

33. Eliasson, P., Andersson, T., Hammerman, M. \& Aspenberg, P. Primary gene response to mechanical loading in healing rat Achilles tendons. J. Appl. Physiol. 114, 1519-1526 (2013).

34. Belmont, L. D. \& Mitchison, T. J. Identification of a protein that interacts with tubulin dimers and increases the catastrophe rate of microtubules. Cell 84, 623-631 (1996).

35. Moore, P. B., Huxley, H. E. \& DeRosier, D. J. Three-dimensional reconstruction of F-actin, thin filaments and decorated thin filaments. J. Mol. Biol. 50, 279-296 (1970).

36. Ridley, A. J. \& Hall, A. The small GTP-binding protein tho regulates the assembly of focal adhesions and actin stress fibers in response to growth factors. Cell 70, 389-399 (1992).

37. Gautreau, A., Poullet, P., Louvard, D. \& Arpin, M. Ezrin, a plasma membrane-microfilament linker, signals cell survival through the phosphatidylinositol 3-kinase Akt pathway. PNAS 96, 7300-7305 (1999).

38. Takahashi, $K$. et al. Direct interaction of the Rho GDP dissociation inhibitor with ezrin/radixin/moesin initiates the activation of the Rho small G protein. J. Biol. Chem. 272, 23371-23375 (1997).

39. Watanabe, N., Kato, T., Fujita, A., Ishizaki, T. \& Narumiya, S. Cooperation between mDia1 and ROCK in Rho-induced actin reorganization. Nat. Cell Biol. 1, 136-143 (1999).

40. Somogyi, K. \& Rørth, P. Evidence for tension-based regulation of Drosophila MAL and SRF during invasive cell migration. Dev. Cell 7, 85-93 (2004).

41. Straight, A. F., Field, C. M. \& Mitchison, T. J. Anillin Binds Nonmuscle Myosin II and Regulates the Contractile Ring. Mol. Biol. Cell 16, 193-201 (2005).

42. Piekny, A. J. \& Glotzer, M. Anillin Is a Scaffold Protein That Links RhoA, Actin, and Myosin during Cytokinesis. Curr. Biol. 18, 30-36 (2008).

43. Vermeulen, S. et al. Identification of topographical architectures supporting the phenotype of rat tenocytes. Acta Biomater. 83, 277-290 (2019).

44. Baek, K. et al. Modulation of actin structure and function by phosphorylation of Tyr-53 and profilin binding. Proc. Natl. Acad. Sci. U. S. A. 105, 11748-11753 (2008).

45. Papakonstanti, E. A. \& Strournaras, C. Association of PI-3 Kinase with PAK1 Leads to Actin Phosphorylation and Cytoskeletal Reorganization. Mol. Biol. Cell 13, 2946-2962 (2002).

46. Moriyama, K. \& Yahara, I. Human CAP1 is a key factor in the recycling of cofilin and actin for rapid actin turnover. J. Cell Sci. 115, 1591-1601 (2002).

47. Sharma, S., Grintsevich, E. E., Phillips, M. L., Reisler, E. \& Gimzewski, J. K. Atomic force microscopy reveals drebrin induced remodeling of F-actin with subnanometer resolution. Nano Lett. 11, 825-827 (2011).

48. Treisman, R. Identification of a Protein-Binding Site That Mediates Transcriptional Response of the c-fos Gene to Serum Factors. Cell 46, 567-574 (1986).

49. Schratt, G. et al. Serum response factor is required for immediate-early gene activation yet is dispensable for proliferation of embryonic stem cells. Mol. Cell. Biol. 21, 2933 (2001).

50. Lejard, V. et al. EGR1 and EGR2 involvement in vertebrate tendon differentiation. J. Biol. Chem. 286, 5855-5867 (2011).

51. Guerquin, M. J. et al. Transcription factor EGR1 directs tendon differentiation and promotes tendon repair. J. Clin. Invest. 123, 3564-3576 (2013).

52. Gaut, L. et al. EGR1 regulates transcription downstream of mechanical signals during tendon formation and healing. PLoS One 11,1-16 (2016).

53. Wilder, P. J., Bernadt, C. T., Kim, J.-H. \& Rizzino, A. Stimulation of the murine type II transforming growth factor-beta receptor promoter by the transcription factor Egr-1. Mol. Reprod. Dev. 63, 282-90 (2002).

54. Zhang, Y., Feng, X.-H. \& Derynck, R. Smad3 and Smad4 cooperate with c-Jun/c-Fos to mediate TFG-b-induced transcription. Nature 394, 909-913 (1998). 
55. Fine, A. \& Goldstein, R. H. The effect of transforming growth factor- $\beta$ on cell proliferation and collagen formation by lung fibroblasts. J. Biol. Chem. 262, 3897-3902 (1987).

56. Brunschwig, E. B. et al. PMEPA1, a transforming growth factor- $\beta$-induced marker of terminal colonocyte differentiation whose expression is maintained in primary and metastatic colon cancer. Cancer Res. 63, 1568-1575 (2003).

57. Poveda, J. et al. MXRA5 is a TGF- $\beta 1$-regulated human protein with anti-inflammatory and antifibrotic properties. J. Cell. Mol. Med. 21, 154-164 (2017).

58. Paliwal, S., Shi, J., Dhru, U., Zhou, Y. \& Schuger, L. P311 binds to the latency associated protein and downregulates the expression of TGF- $\beta 1$ and TGF- $\beta 2$. Biochem. Biophys. Res. Commun. 315, 1104 1109 (2004).

59. Stopa, M. et al. Participation of Smad2, Smad3, and Smad4 in Transforming Growth Factor B ( TGF-B)-induced Activation of Smad7. J. Biol. Chem. 275, 29308-29317 (2000).

60. Agassandian, M. et al. VCAM-1 is a TGF- $\beta 1$ Inducible Gene Upregulated in Idiopathic Pulmonary Fibrosis. Cell Signal. 27, 2467-2473 (2015).

61. Jones, N. \& Agani, F. H. Hyperoxia induces Egr-1 expression through activation of extracellular signal-regulated kinase 1/2 pathway. J. Cell. Physiol. 196, 326-333 (2003).

62. Abdel-Latif, M. M. M., Windle, H. J., Davies, A., Volkov, Y. \& Kelleher, D. A new mechanism of gastric epithelial injury induced by acid exposure: the role of Egr-1 and ERK signaling pathways. J. Cell. Biochem. 108, 249-260 (2009).

63. Skalli, O. et al. A monoclonal antibody against $\alpha$-smooth muscle actin: A new probe for smooth muscle differentiation. J. Cell Biol. 103, 2787-2796 (1986).

64. Bi, W., Deng, J. M., Zhang, Z., Behringer, R. R. \& De Crombrugghe, B. Sox9 is required for cartilage formation. Nat. Genet. 22, 85-89 (1999).

65. Inman, G. J. et al. SB-431542 is a potent and specific inhibitor of transforming growth factor- $\beta$ superfamily type I activin receptor-like kinase (ALK) receptors ALK4, ALK5, and ALK7. Mol. Pharmacol. 62, 65-74 (2002).

66. Sit, S. T. \& Manser, E. Rho GTPases and their role in organizing the actin cytoskeleton. J. Cell Sci. 124, 679-683 (2011).

67. Hutchings, K. et al. Pharmacokinetic Optimization of CCG-203971: Novel Inhibitors of the Rho/MRTF/SRF Transcriptional Pathway as Potential Antifibrotic Therapeutics for Systemic Scleroderma. Bioorg Med Chem Lett. 27, 1744-1749 (2017).

68. Limouze, J., Straight, A. F., Mitchison, T. \& Sellers, J. R. Specificity of blebbistatin, an inhibitor of myosin II. J. Muscle Res. Cell Motil. 25, 337-341 (2004).

69. Murthy, K. \& Wadsworth, P. Myosin-II-dependent localization and dynamics of F-actin during cytokinesis. Curr. Biol. 15, 724-731 (2005).

70. Subramanian, A. et al. A Next Generation Connectivity Map: L1000 Platform and the First 1,000,000 Profiles. Cell 171, 1437-1452.e17 (2017).

71. Larsson, C. Protein kinase C and the regulation of the actin cytoskeleton. Cell. Signal. 18, 276284 (2006).

72. Schliwa, M., Nakamura, T., Porter, K. R. \& Euteneuer, U. A tumor promoter induces rapid and coordinated reorganization of actin and vinculin in cultured cells. J. Cell Biol. 99, 1045-1059 (1984).

73. Soh, J.-W., Lee, E. H., Prywes, R. \& Weinstein, I. B. Novel Roles of Specific Isoforms of Protein Kinase C in Activation of the c- fos Serum Response Element. Mol. Cell. Biol. 19, 1313-1324 (1999).

74. Halvorson, L. M., Kaiser, U. B. \& Chin, W. W. The protein kinase C system acts through the early growth response protein 1 to increase LH $\beta$ gene expression in synergy with steroidogenic factor- 1 . Mol. Endocrinol. 13, 106-116 (1999).

75. Shields, L. B. E. et al. Adverse effects associated with high-dose recombinant human bone morphogenetic protein-2 use in anterior cervical spine fusion. Spine (Phila. Pa. 1976). 31, 542-547 (2006).

76. Schwarz, C. et al. Mechanical load modulates the stimulatory effect of BMP2 in a rat nonunion model. Tissue Eng. - Part A 19, 247-254 (2013).

77. Weis, S. M. \& Cheresh, D. A. Pathophysiological consequences of VEGF-induced vascular permeability. Nature 437, 497-504 (2005).

78. Zhu, J. et al. The regulation of phenotype of cultured tenocytes by microgrooved surface structure. Biomaterials 31, 6952-6958 (2010). 
79. Kapoor, A., Caporali, E. H. G., Kenis, P. J. A. \& Stewart, M. C. Microtopographically patterned surfaces promote the alignment of tenocytes and extracellular collagen. Acta Biomater. 6, 2580-2589 (2010).

80. English, A. et al. Substrate topography: A valuable in vitro tool, but a clinical red herring for in vivo tenogenesis. Acta Biomater. 27, 3-12 (2015).

81. Tong, W. Y. et al. Functional replication of the tendon tissue microenvironment by a bioimprinted substrate and the support of tenocytic differentiation of mesenchymal stem cells. Biomaterials 33, 7686-7698 (2012).

82. Zhou, K., Feng, B., Wang, W., Zhang, W. \& Zhou, G. Nanoscaled and microscaled parallel topography promotes tenogenic differentiation of ASC and neotendon formation in vitro. Int. J. Nanomedicine 3867-3881 (2018).

83. Wang, W. et al. Induction of predominant tenogenic phenotype in human dermal fibroblasts via synergistic effect of TGF- $\beta$, and elongated cell shape. Am J Physiol Cell Physiol 310, C357-C372 (2015).

84. Yin, Z. et al. The regulation of tendon stem cell differentiation by the alignment of nanofibers. Biomaterials 31, 2163-2175 (2010).

85. Barber, J. G., Handorf, A. M., Allee, T. J. \& Li, W. J. Braided nanofibrous scaffold for tendon and ligament tissue engineering. Tissue Eng. - Part A 19, 1265-1274 (2013).

86. Sharma, R. I. \& Snedeker, J. G. Biochemical and biomechanical gradients for directed bone marrow stromal cell differentiation toward tendon and bone. Biomaterials 31, 7695-7704 (2010).

87. Park, J. S. et al. The Effect of Matrix Stiffness on the Differentiation of Mesenchymal Stem Cells in Response to TGF- $\beta$. Biomaterials 32, 3921-3930 (2012).

88. Leight, J. L., Wozniak, M. A., Chen, S., Lynch, M. L. \& Chen, C. S. Matrix rigidity regulates a switch between TGF-B1-induced apoptosis and epithelial-mesenchymal transition. Mol. Biol. Cell 23, 781791 (2012).

89. Allen, J. L., Cooke, M. E. \& Alliston, T. ECM stiffness primes the TGFB pathway to promote chondrocyte differentiation. Mol. Biol. Cell 23, 3731-3742 (2012).

90. Kuo, C. K. \& Tuan, R. S. Mechanoactive tenogenic differentiation of human mesenchymal stem cells. Tissue Eng. - Part A. 14, 1615-1627 (2008).

91. Morita, Y., Watanabe, S., Ju, Y. \& Xu, B. Determination of optimal cyclic Uniaxial stretches for stem cell-to-tenocyte differentiation under a wide range of mechanical stretch conditions by evaluating gene expression and protein synthesis levels. Acta Bioeng. Biomech. 15, 71-79 (2013).

92. Huisman, E., Lu, A., McCormack, R. G. \& Scott, A. Enhanced collagen type i synthesis by human tenocytes subjected to periodic in vitro mechanical stimulation. BMC Musculoskelet. Disord. 15, 1-8 (2014).

93. Maeda, T. et al. Conversion of Mechanical Force into TGF-b-Mediated Biochemical Signals. Curr. Biol. 21, 933-941 (2011).

94. Grier, W. K., Moy, A. S. \& Harley, B. A. C. Cyclic tensile strain enhances human mesenchymal stem cell SMAD 2/3 activation and tenogenic differentiation in anisotropic collagen-glycosaminoglycan scaffolds. Eur. Cells Mater. 33, 227-239 (2017).

95. Furumatsu, T. et al. Tensile strain increases expression of CCN2 and COL2A1 by activating TGF- $\beta-S m a d 2 / 3$ pathway in chondrocytic cells. J. Biomech. 46, 1508-1515 (2013).

96. Kuwahara, K., Barrientos, T., Pipes, G. C. T., Li, S. \& Olson, E. N. Muscle-Specific Signaling Mechanism That Links Actin Dynamics to Serum Response Factor. Mol. Cell. Biol. 25, 3173-3181 (2005). 97. Tsai-Morris, C. H., Cao, X. \& Sukhatme, V. P. 5 ' Flanking Sequence and Genomic Structure of Egr-1, a Murine Mitogen Inducible Zinc Finger Encoding Gene. Nucleic Acids Res. 16, 8835-8846 (1988). 98. $\mathrm{Xu}, \mathrm{B}$. et al. RhoA/ROCK, cytoskeletal dynamics, and focal adhesion kinase are required for mechanical stretch-induced tenogenic differentiation of human mesenchymal stem cells. J. Cell. Physiol. 227, 2722-2729 (2012).

99. Wang, J. et al. Smooth muscle actin determines mechanical force-induced p38 activation. J. Biol. Chem. 280, 7273-7284 (2005).

100. Johnson, L. A. et al. Novel Rho/MRTF/SRF Inhibitors Block Matrix-stiffness and TGF- $\beta$ Induced Fibrogenesis in Human Colonic Myofibroblasts. Inflamm Bowel Dis. 20, 154-165 (2014).

101. Gualdrini, F. et al. SRF Co-factors Control the Balance between Cell Proliferation and Contractility. Mol. Cell 64, 1048-1061 (2016). 
102. Wesner, M. et al. A Pilot Study Evaluating the Effectiveness of Platelet-Rich Plasma Therapy for Treating Degenerative Tendinopathies: A Randomized Control Trial with Synchronous Observational Cohort Setting. PLoS One (2016). doi:10.1371/journal.pone.0147842

103. Zhao, Y. et al. High-definition micropatterning method for hard, stiff and brittle polymers. Mater. Sci. Eng. C 71, 558-564 (2017).

104. McQuin, C. et al. CellProfiler 3.0: Next-generation image processing for biology. PLoS Biol. 16, 1-17 (2018).

105. Schindelin, J. et al. Fiji - an Open platform for biological image analysis. Nat. Methods 9, 241 (2009).

\section{Supplementary Figures}



Supplementary Figure 1: STRING gene network representation illustrating RhoA interconnectedness with cytoskeletal genes. Every dot represents a DEG in the gene network. Red dots are DEGs associated with the GO term "cytoskeletal organization". For illustration purposes, RhoA was included in the network to demonstrate the strong relationship with cytoskeletal associated genes.

A

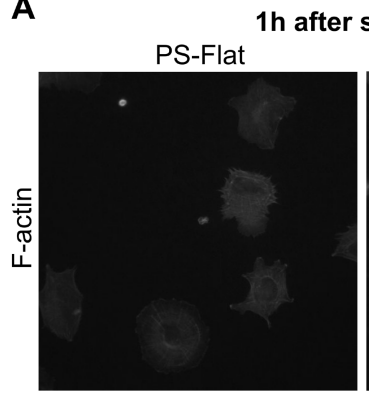
1h after seeding

\section{B}


Supplementary Figure 2: Early attachment of MSCs on microtopographies is characterized by increased F-actin levels. A) Immunolabeling by phalloidin allows visualizing F-actin in MSCs. During the early phase of cell attachment MSCs engulf the micro-topographies which coincides with increased F-actin levels. B) Quantifying F-actin of MSCs across multiple time points reveals that strongest intensities are detected on the PS-1018 surface $1 \mathrm{~h}$ after cell seeding after which intensity levels declines (*** $\mathrm{P}<0.001)$. Scale bar represents $100 \mu \mathrm{m}$. 
A

\begin{tabular}{l|l} 
Accession & \multicolumn{1}{|c}{ Name } \\
\hline P60709 & Actin, cytoplasmic 1 \\
Q01518-1 & Adenylyl cyclase-associated protein 1 \\
Q16643-3 & Isoform 3 of Drebrin
\end{tabular}

B

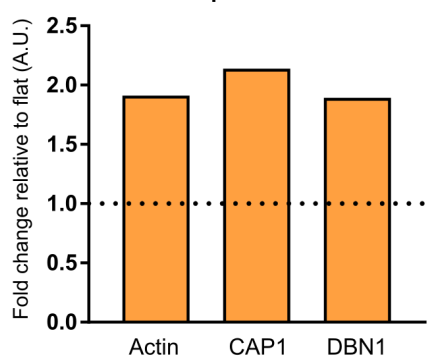

Supplementary Figure 3: Phospho proteomics on MSCs after 1 h of cell culture on PS-1018 reveals active cytoskeletal remodeling. A) Phospho proteomics was able to detect both the phosphorylated and non-phosphorylated form of actin, adenylyl cyclase-associated protein 1, and isoform of drebrin 3. B) P-Proteomics reveals increased levels of phosphorylated actin and the actin associated proteins CAP1 and DBN1 (n=1).

A
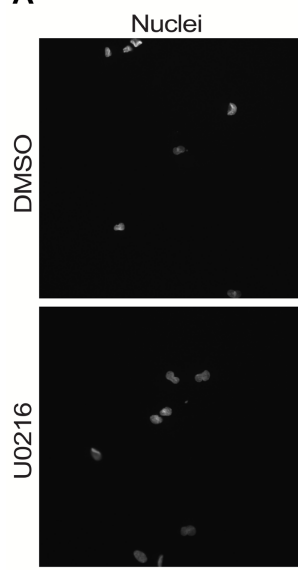
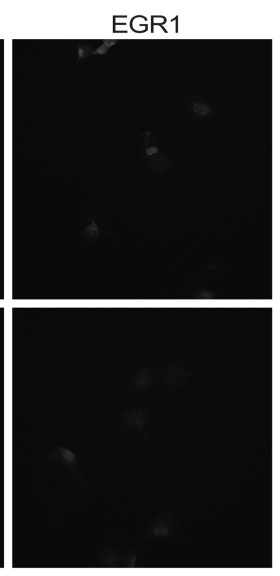

B

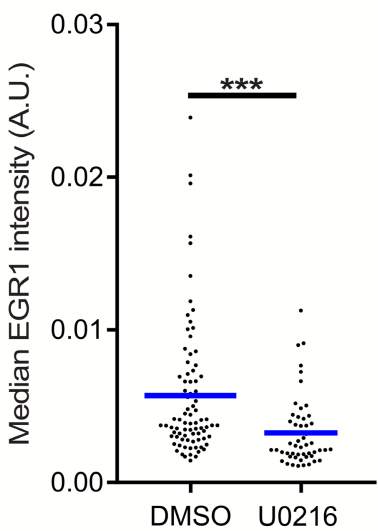

Supplementary Figure 4: MEK1/2 inhibitors downregulate EGR1 levels. AB) Quantifying the EGR1 immunolabeling signal reveals that the MEK1/2 inhibitor reduces EGR1 levels $2 \mathrm{~h}$ after cell seeding on PS-Flat $(* * * \mathrm{P}<0.001)$. 


\section{Tubulin inhibitor perturbagen class score}

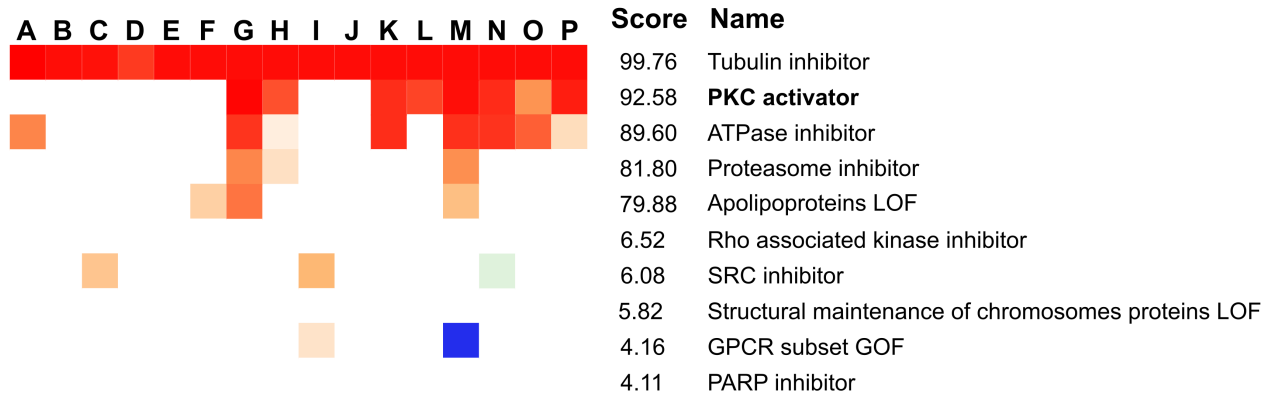

\section{Legend}
A ABT-751
I Paxlitacel
B Albendazole
J Parbendazole
C Docetaxe
K Podophyllotoxin
D Epithilone
L SA-792574
E Fenbedazole
M Vinblastidine
F Flubendazole
N Vinchristine
G Mebendazole
O Vindesin
H Oxibendazole
P Vinorelbine

Supplementary Figure 5: Tubulin inhibitor perturbagen class score. From this list, we notice the presence of PKC activators as inducing gene signatures similar as tubulin inhibitors.

\section{ACTB Compound class score}

$\begin{array}{lll}\text { Score } & \text { Name } & \text { Description } \\ 99.95 & \text { VU-0418946-1 } & \text { hypoxia inducible factor activator } \\ 99.95 & \text { cucurbitacin-i } & \text { inhibitor of STAT3/JAK2 signaling } \\ 99.92 & \text { VU-0418947-2 } & \text { hypoxia inducible factor activator } \\ 99.87 & \text { cytochalasin-d } & \text { actin polymerization inhibitor, actin stabilizer } \\ 99.13 & \text { phorbol-12-myristate-13-acetate } & \text { PKC activator, CD antagonist } \\ 99.04 & \text { JTC-801 } & \text { opioid receptor antagonist } \\ 98.87 & \text { cytochalasin-b } & \text { microtubule inhibitor, phagocytosis inhibitor } \\ 98.8 & \text { quinoclamine } & \text { algicide } \\ 97.31 & \text { ingenol } & \text { PKC activator } \\ 97.16 & \text { obatoclax } & \text { BCL inhibitor, MCL1 inhibitor } \\ 82.55 & \text { U-46619 } & \text { thromboxane receptor agonist } \\ 62.59 & \text { SJ-172550 } & \text { MDM inhibitor } \\ 48.44 & \text { cobalt(II)-chloride } & \text { HSP agonist } \\ 35.14 & \text { VX-222 } & \text { HCV inhibitor, RNA-directed RNA polymerase inhibitor } \\ 31.01 & \text { BRD-A80383043 } & \text { glutamate receptor agonist, glutamate receptor antagonist } \\ 24.65 & \text { 1-monopalmitin } & \text { P glycoprotein inhibitor } \\ 1.13 & \text { PF-04217903 } & \text { C-Met inhibitor, hepatocyte growth factor receptor inhibitor } \\ -5.63 & \text { PKCbeta-inhibitor } & \text { PKC inhibitor } \\ -6.35 & \text { ranitidine } & \text { histamine receptor antagonist } \\ -30.91 & \text { GW-7647 } & \text { PPAR receptor agonist } \\ & & \end{array}$

Supplementary Figure 6: ACTB compound class score. PKC activators ingenol and phorbol-12-myristate-13-acetate are part of the list together with cytochalasin-d, an actin polymerization inhibitor, and cytochalisin-b, a microtubuline inhibitor. These inhibitors give rise to similar gene signature patterns in $A C T B$ knockdown cell lines. 




Supplementary Figure 7: EGR1 genetic connectivity list. From this list, it is clear that there is a strong relationship between EGR1 and the TGF- $\beta$ R-II.

\section{PKC activator compound score}

\begin{tabular}{|lll} 
A B C Score & Name & Description \\
99.93 & phorbol-12-myristate-13-acetate & PKC activator, CD antagonist \\
99.93 & ingenol & PKC activator \\
99.89 & prostratin & NFkB pathway activator, PKC activator \\
99.75 & GR-127935 & serotonin receptor antagonist \\
99.75 & obatoclax & BCL inhibitor, MCL1 inhibitor \\
99.72 & VU-0418947-2 & hypoxia inducable factor activator \\
99.68 & JTC-801 & opioid receptor antagonist \\
99.58 & QS-11 & ARFGAP inhibitor \\
99.58 & VU-0418946-1 & hypoxia inducable factor activator \\
99.54 & malonoben & protein tyrosine kinase inhibitor, tyrosine kinase inhibitor \\
-74.52 & VER-155008 & HSP inhibitor \\
-80.01 & UNC-0321 & histone lyinse methyltransferase inhibitor \\
-84.04 & LY-364947 & TGF beta receptor inhibitor, p38 MAPK inhibitor \\
-86.53 & TWS-119 & glycogen synthase kinase inhibitor \\
-87.9 & MEK1-2-inhibitor & MEK inhibitor \\
-89.27 & PD-184352 & MEK inhibitor, MAP kinase inhibitor \\
-91.51 & AS-703026 & MEK inhibitor \\
-91.8 & AS-605240 & Phosphatidylinositol 3-kinase (PI3K) inhibitor, PI3K inhibitor \\
-92.7 & PD-98059 & MEK inhibitor, MAP kinase inhibitor \\
-95.28 & PD-0325901 & MEK inhibitor, MAP kinase inhibitor, protein kinase inhibitor \\
\hline
\end{tabular}

Supplementary Figure 8: PKC activator compound scores. From this list, we selected ingenol and prostratin to assess their potential to replace micro-topographical induced signaling. MAPK and TGF$\beta R-I I$ inhibitors induced opposite gene signatures. 
A

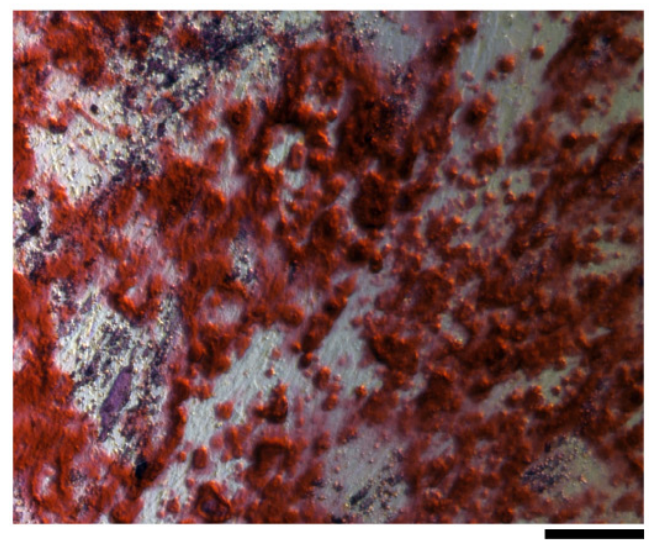

B

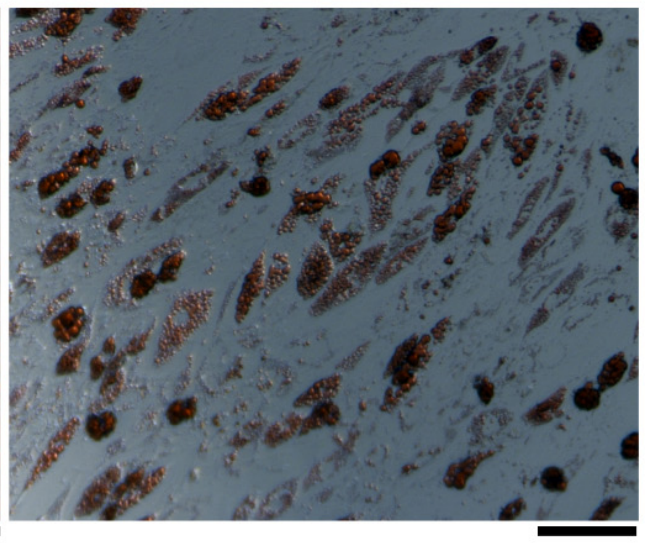

Supplementary Figure 9: Assessment of AD-MSC multipotency. A) After osteogenic induction, mineralized calcium deposits were formed as visualized by Alizarin Red staining. B) After adipogenic induction, oil droplets were formed as visualized by Oil Red O staining. Scale bar represent $100 \mu \mathrm{m}$. 


\section{Chapter VI}

\section{The Covalent State of Immobilized}

RGD Peptides dictates the

\section{Mechanobiological Response of}

\section{Fibroblasts}

$\underline{\text { Gülistan Koçer }}{ }^{a, \dagger}, \underline{\text { Steven Vermeulen }}{ }^{\text {b,c, }}$, Jenny Brinkmannn, Laura BM Gamo, Dennie Hebels ${ }^{\mathrm{b}}$, Aliaksei Vasilevich ${ }^{\mathrm{c}}$, Jeroen van de Peppel $^{\mathrm{d}}$, Shrikrishnan Sankaran ${ }^{a}$, Emanuela Cavatorta ${ }^{a}$, Johannes van Leeuwen ${ }^{\mathrm{d}}$, Jan de Boer ${ }^{\mathrm{c}}$ and Pascal Jonkheijm ${ }^{\mathrm{a}}$

a Laboratory of Biointerface Chemistry, TechMed Centre and MESA+ Institute for Nanotechnology, University of Twente, Enschede, the Netherlands

b Laboratory for Cell Biology-Inspired Tissue Engineering, MERLN Institute, University of Maastricht, Maastricht, The Netherlands

${ }^{\mathrm{c}}$ BioInterface Science Group, Department of Biomedical Engineering, University of Eindhoven, Eindhoven, The Netherlands

d Department of Internal Medicine, Erasmus University Medical Centre, Rotterdam, the Netherlands

†'These authors contributed equally.

Manuscript in preparation 


\section{Abstract}

Integrin-matrix interactions provide essential means of communication between the cell and the surrounding environment. A challenge in the tissue-engineering field is recreating these conditions in the culture dish for optimally controlling cell behavior. Current approaches include the use of matrix coatings or printed peptide sequences such as RGDs that are biologically active against integrin receptor proteins. Implementing these concepts in the culture environment are known for inducing beneficial effects on cell behavior. However, these approaches fail to recapitulate the dynamic physiological environment. Supramolecular surfaces through cucurbit[8]uril chemistry can allow such a dynamical environment through the non-covalent presentation of RGD peptides. In this study, we compare how this dynamic presentation influences cell behavior compared to static RGD that is covalently bound to the surface and conventional fibronectin coatings. We found that these surfaces induced distinct focal adhesion and morphological profiles in fibroblasts. Furthermore, non-covalent RGD presentation induced a more robust mechanobiological response compared to covalent bound RGD and regular fibronectin, as shown by higher levels of the mechanosensitive transcription factors SRF and EGR-1. This study provides a more in-depth understanding on cell response to noncovalent surfaces while substantiating the high potential of these surfaces as better biomimetic models for the culture dish. 


\section{Introduction}

Cell adhesion to the natural extracellular matrix (ECM) is largely mediated by a family of adhesion receptors, the integrins. These transmembrane receptors recognize and interact with specific sequences in ECM proteins (i.e., ligands) and transmit signals to the intracellular space acting as a signaling platform ${ }^{1}$. Integrin-mediated adhesions are tightly linked to the actin cytoskeleton through integrin-cytoskeleton linker proteins such as talin and vinculin, directing actin assembly as well as fibrillar (F)-actin organization and dynamics ${ }^{2-4}$. Furthermore, they are able to regulate cellular processes such as migration, morphology, and signaling events such as transcriptional activity and, eventually, cellular fate and function ${ }^{2,5-8}$. The ECM derived amino acid sequence Arg-Gly-Asp-Ser (RGDS) is the minimal adhesion domain of fibronectin ${ }^{9}$ and has been employed to introduce integrin-mediated effects on a large variety of surface engineered systems for controlling cell behavior ${ }^{10-12}$. Seminal studies by Mrksich and co-workers made use of self-assembled monolayers (SAMs) of alkanethiolates on gold to covalently immobilize RGD ligands. Variations in the ligand affinity towards integrins (i.e., linear vs. cyclic RGD) as well as ligand density on these surfaces had critical effects on cell adhesion and spreading, focal adhesion development, gene expression, and differentiation in cells ${ }^{13-15}$. Furthermore, precise control regarding the positioning and spacing of the RGD ligands, as achieved by Spatz and co-workers on gold nanoparticles, revealed that a critical ligand spacing exists for focal adhesion activation, and enabled studying the effect of ligand (dis)order and immobilization chemistry on cell adhesion and migration ${ }^{16-20}$. These and many other studies have been essential in advancing the design of cell-instructive biointerfaces and to reveal the mechanisms of integrin-mediated cell adhesion as well as cellular fate $10,12,19,21-23$.

Nevertheless, these biointerfaces introduce a static presentation of peptide ligands, in contrast to the dynamic native microenvironment, where instructive cues are presented in a spatiotemporally regulated fashion ${ }^{1024}$. Therefore, incorporation of bioinspired dynamic cues has become an important focus in the design of new biomimetic cell-instructive biointerfaces. Dynamic biointerfaces have been achieved using static ligands where the specific ligand or the surrounding groups were chemically modified with stimuli-responsive elements (e.g., responsive to light and electrochemistry) ${ }^{21,25-30}$. For example, when a light-responsive azobenzene group was introduced to the RGD molecular backbone, ligand availability for integrin binding could be reduced by light-induced trans-to-cis conformational changes of the azobenzene molecules, thereby reversibly preventing cell adhesion 27,30. Yousaf and co-workers have achieved reversibility in the conformation of RGD ligands between linear and cyclic RGD by immobilizing them with redox reactive hydroquines ${ }^{28}$. Furthermore, del Campo and co-workers have developed light-reactive integrin ligands providing dynamic control over ligand availability for influencing cell adhesion and migration ${ }^{26,31}$.

A step further are supramolecular systems, which rely on non-covalent interactions for assembling molecular components, introduce an elegant way for achieving highly biomimetic and dynamic display of bioactive ligands ${ }^{32-35}$. Due to the non-covalent and dynamic nature of 
interactions forming these biointerfaces, they have been suggested to better mimic the ECM 10,36-38. In this context, supramolecular systems that use host-guest interactions introduce a powerful tool for designing dynamic biointerfaces, where the affinity between the host and ligand conjugated guest molecules is tunable $33,34,39,40$. For example, Stupp and coworkers linked the $\beta$-cyclodextrin $(\beta$-CD) host molecule to alginate matrix and used guest molecules naphthoic acid and adamantane for the functionalization of RGDS and non-functional RGES peptides, respectively. Surfaces could be switched from cell adhesive (RGD-surfaces) to cell repellent (RGES-surfaces), due to the higher affinity of adamantane for $\beta$-CD competing with naphtoic acid for binding ${ }^{41}$. In this context, we and others have also demonstrated the possibility to reversibly control cell adhesion by control of light and electrochemical switching on host-guest supramolecular biointerfaces ${ }^{40,42}$.

Recently, we employed a supramolecular cucurbit[8]uril (CB[8])-based host-guest system displaying RGD ligands to cells for studying dynamic cell-generated adhesive forces in comparison to static surfaces ${ }^{36}$. Dynamic surfaces were prepared by first conjugating a thiolated methyl viologen $\left(\mathrm{MV}^{2+}\right)$, that can bind to $\mathrm{CB}[8]$ as the first guest, to the maleimide groups on a SAM. Then, a naphthyl modified RGD (Np-RGD) was introduced as the second guest for binding to $\mathrm{MV}^{2+}$. $\mathrm{CB}[8]$ binary complex, resulting in a ternary complex of $\mathrm{MV}^{2+} \cdot \mathrm{CB}[8] \cdot \mathrm{Np}-$ RGD. For covalent surfaces, a cysteine bearing RGD peptide was directly conjugated to the maleimide groups on the surface. Utilizing this platform, we showed that non-covalent surfaces performed as well as covalent surfaces in sustaining cell adhesion and presented a highly versatile and chemically controlled way to generate new dynamic cell-instructive biointerfaces ${ }^{36}$.

In this study, we employ these non-covalent RGD presenting CB[8]-based surfaces for determining if this ligand presentation alters the behavior of fibroblasts, a cell type that is extensively used for integrin-mediated cell adhesion studies ${ }^{19}$, compared to static covalent surfaces and standard fibronectin coatings. Each of these surfaces induced a distinct mechanobiological profile in fibroblasts, which was visible due to altered morphological characteristics, actin and focal adhesion organization, and gene expression. Non-covalent RGD presenting surfaces induced the strongest response concerning F-actin assembly, which coincided with the expression of mechanosensitive transcription factors such as EGR-1 and SRF. In conclusion, this study provides a more in-depth understanding on cell response to noncovalent surfaces, while substantiating the high potential of these surfaces as better biomimetic models to develop new biointerfaces for biomaterial development and platforms for fundamental cell biological studies 


\section{Results}

\section{Surface analysis of covalent and non-covalent RGD functionalization}

In earlier work, we developed a surface functionalization strategy, where mixed self-assembled monolayers of tetra(ethylene glycol) and maleimide (Mal-EG4) were employed as a base layer to further functionalize gold surfaces with the cell-adhesive RGD peptide via covalent $\left(\mathrm{COV}_{\mathrm{RGD}}\right)$ or non-covalent (supramolecular) (NON_COV $\mathrm{RGD}_{\text {) }}$ approaches ${ }^{36}$. In the $\mathrm{COV}_{\mathrm{RGD}}$ approach, a cysteine bearing RGD (COV $\mathrm{RGD})$ was directly coupled to $\mathrm{Mal}_{-\mathrm{EG}_{4}}$ monolayers. In order to achieve NON_COV RGD surfaces, a ternary complex of a cucurbit[8]uril (CB[8]) host and two aromatic guests, i.e., thiolated methylviologen $\left(\mathrm{MV}^{2+}\right)$ and naphthol-coupled RGD (NON_COV $V_{\text {RGD }}$ ) peptide, were assembled onto the Mal-EG monolayers $^{36}$ (Figure 1). It is important to note that, in this system, the molar density of $\mathrm{Mal}_{-\mathrm{EG}} \mathrm{G}_{4}$ corresponds to ligand density (i.e., RGD density) that is used for cell experiments.

The formation of both monolayers was validated with Fourier-transform infrared reflectionabsorption spectroscopy (FT-IRRAS) (Figure S1). For NON_COV RGD surfaces, peaks of $\mathrm{CB}$ [8] can be observed at $1746 \mathrm{~cm}^{-1}(\mathrm{C}-\mathrm{N})$ and $1470 \mathrm{~cm}^{-1}(\mathrm{C}=\mathrm{O})$, while these peaks were absent on $\mathrm{COV}_{\mathrm{RGD}}$. The peptide peaks were visible on both SAMs around $3300 \mathrm{~cm}^{-1}(\mathrm{~N}-\mathrm{H})$ as well as $1670(\mathrm{C}=\mathrm{N}), 1544(\mathrm{C}-\mathrm{N})$, and $1270 \mathrm{~cm}^{-1}(\mathrm{C}-\mathrm{OH})^{43}$. Peaks in the region between $1195 \mathrm{~cm}^{-1}$ and $1375 \mathrm{~cm}^{-1}$ are characteristic for single bond stretching of C-N, C-C and C-O bonds, found in $\mathrm{CB}[8]$ as well as in $\mathrm{MV}^{2+}$ and $\mathrm{EG}_{4}$ alkane chains. Peaks between $2929 \mathrm{~cm}^{-1}$ and $2865 \mathrm{~cm}^{-1}$ are characteristic for $\mathrm{C}-\mathrm{H}$ stretching vibrations and can be assigned to alkane chains in $\mathrm{Mal}_{-} \mathrm{EG}_{4}$ in both NON_COV $\mathrm{RGD}_{\text {and }}$ COV $\mathrm{RGD}$.

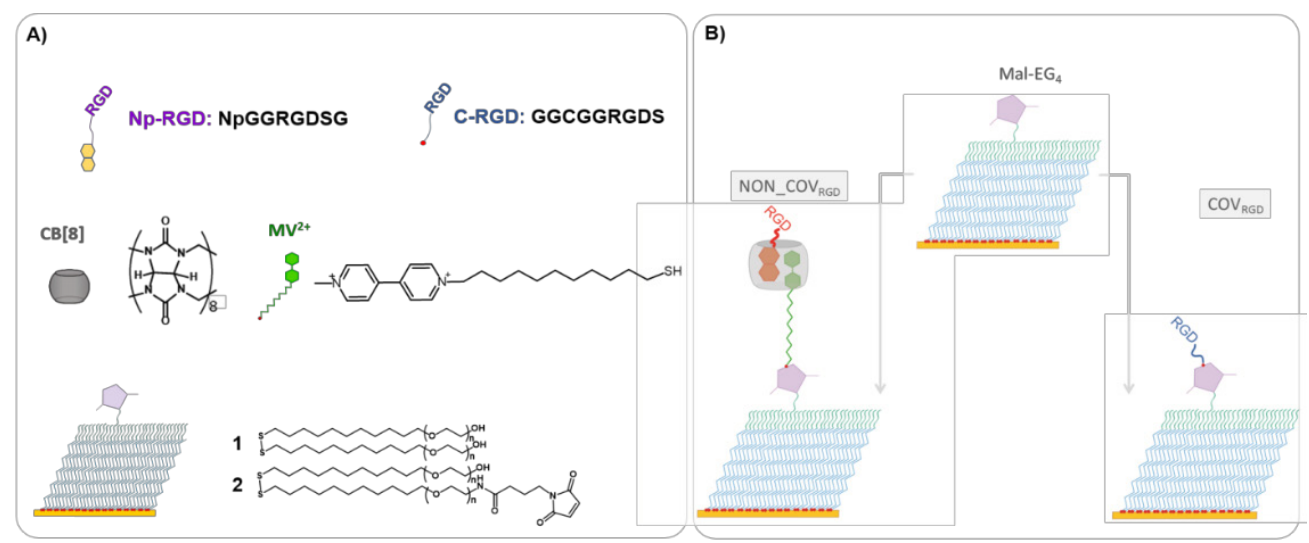

Figure 1: Fabrication scheme. Supramolecular (non-covalent, host-guest) vs. covalent presentation of RGD peptides on thiolated ethylene glycol (EG4) based self-assembled monolayers (SAMs) on gold. A) Components and $\mathbf{B}$ ) surface assembly for NON_COV $\mathrm{RGD}_{\text {and }}$ COV $\mathrm{RGD}_{\mathrm{R}}$ on the surfaces. 


\section{Focal adhesions mature faster on non-covalently bound RGD ligands and fibronectin compared to covalently bound RGD ligands}

In order to have a deeper understanding on how integrin-mediated adhesions are regulated on $\mathrm{COV}_{\mathrm{RGD}}$ and NON_COV RGD surfaces, focal adhesion (FA) dynamics (by studying the localization of focal adhesion protein vinculin in time) ${ }^{3}$ was investigated in response to ligand density, as one extensively studied parameter on traditional covalent surfaces ${ }^{14,23,44}$. Since ligand availability and presentation have been linked to changes in cellular morphology, we also studied F-actin reorganization and cell spreading. Fibronectin coated glass substrates (FN, at $10 \mu \mathrm{g} / \mathrm{mL}$ ), a well-established surface for adhesion studies, were used as a reference surface ${ }^{3,4}$.

Vinculin localization and actin organization (cell spreading) on the different surfaces were studied at 15, 30, 45, 60, and 120min after cell seeding (Figure 2). On FN surfaces, small dotlike vinculin localization (resembling nascent adhesions) was already observed at 15 min, while cells remained mostly spherical as characteristics of initial stages of cell adhesion and spreading 24,45,46. At 30min, distinct vinculin localization in elongating focal adhesions was seen primarily in protruding lamellipodia of spreading cells. Over 45, 60, and 120min, the cell elongation and spreading slowly matured. More visibly, actin stress fibers became more prominent throughout this time, and vinculin adhesive assemblies matured into qualitatively more elongated shapes found throughout the cell, characteristic of FA maturation ${ }^{24}$.

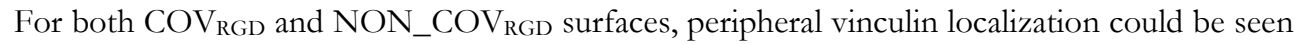
at $30 \mathrm{~min}$ (Figure 3), which acquired more distinguishable structures at $45 \mathrm{~min}$. Interestingly, at 45 min, NON_COV $V_{\text {RGD }}$ surfaces induced more qualitatively elongated focal adhesion (vinculin) structures compared to the cells on $\mathrm{COV}_{\mathrm{RGD}}$ surfaces (with mostly dot-like focal adhesions).

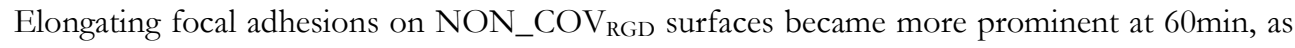
they matured further, a state that was visible on $\mathrm{COV}_{\mathrm{RGD}}$ surfaces only at $120 \mathrm{~min}$. Altogether,

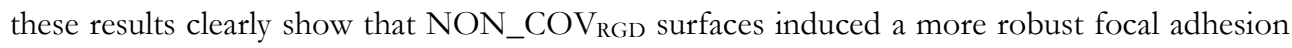
development in time, compared to $\mathrm{COV}_{\mathrm{RGD}}$ surfaces, albeit following a slower development

$15 \mathrm{~min}$

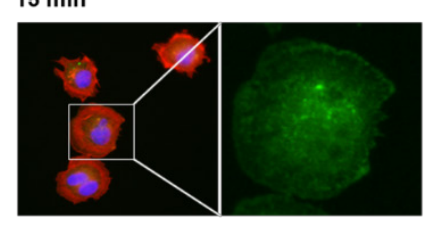

$30 \mathrm{~min}$

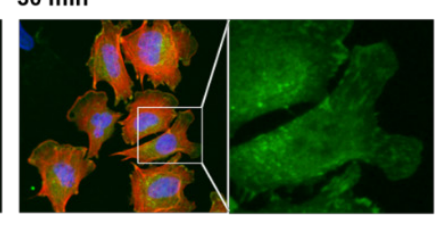

$120 \mathrm{~min}$

$60 \mathrm{~min}$
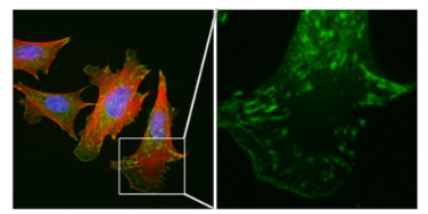

$45 \mathrm{~min}$

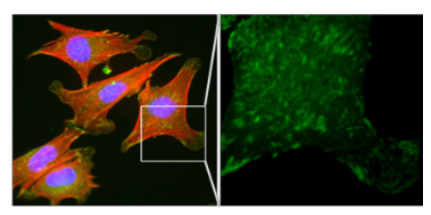

Figure 2: Fluorescence micrographs of NIH3T3 fibroblasts cultured on glass coated with 10 $\mu \mathrm{g} / \mathrm{mL}$ fibronectin at different time points. Vinculin localization became visible at $15 \mathrm{~min}$. Red: Factin, green: vinculin and blue: nucleus. Magnified images show the vinculin staining in more detail. 
compared to FN surfaces. Nevertheless, a more profound clustering of vinculin in these focal adhesions was present on the NON_COV RGD surface, which hints at the possibility of stronger FAK signaling.


Figure 3: Distinct regulation of focal adhesion dynamics in NIH3T3 fibroblasts cultured on $\mathbf{C O V}_{\mathbf{R G D}}$ and NON_COV $\mathbf{R G D}_{\text {RG }}$ surfaces. Red: F-actin, green: vinculin and blue: nucleus. Magnified images show the vinculin staining in more detail. 


\section{The (non-)covalent state of RGD surfaces induces distinct morphological characteristics and F-actin development dynamics in NIH3T3 fibroblasts}

After determining that these surfaces induce differences in focal adhesion formation and dynamics, we wanted to investigate if cell shape characteristics, an important determinant of cell behavior ${ }^{47,48}$, was altered as well. We, therefore, seeded NHT3T fibroblasts on FN, COV RGD, $_{\text {, }}$ and the NON_COV RGD surfaces and cultured them for 25min, 1h, 2h, and $4 \mathrm{~h}$ (Fig. 4). Afterward, cells were fixed and fluorescently labeled with F-actin and DAPI for cytoskeleton and nuclear visualization. Images were analyzed through CellProfiler ${ }^{49}$, which allowed detailed quantification of morphological characteristics on the single-cell level. Principal component analysis revealed that the parameterization of morphological parameters could distinguish cells grown on fibronectin, $\mathrm{COV}_{\mathrm{RGD}}$, and NON_COV $\mathrm{RGD}$ at different time points (Fig. S2).

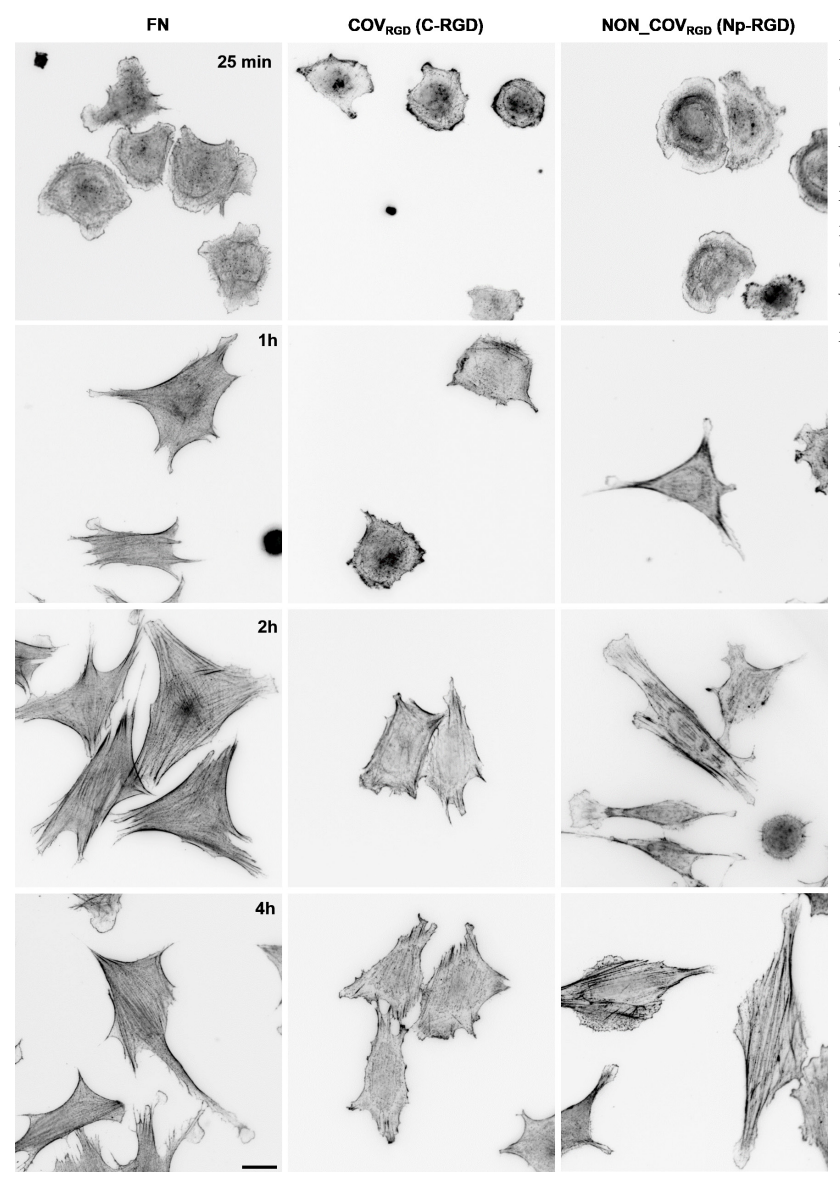

Figure 4. F-actin development dynamics of NIH3T3 fibroblasts cultured on the different surfaces. Visual observations reveals a latency in cell spreading and a round cellular morphology for NIH3T3 fibroblasts cultured on $\mathrm{COV}_{\mathrm{RGD}}$. F-actin staining was accomplished through Phalloidin immunolabeling. Scale bar represents $100 \mu \mathrm{m}$. 
A

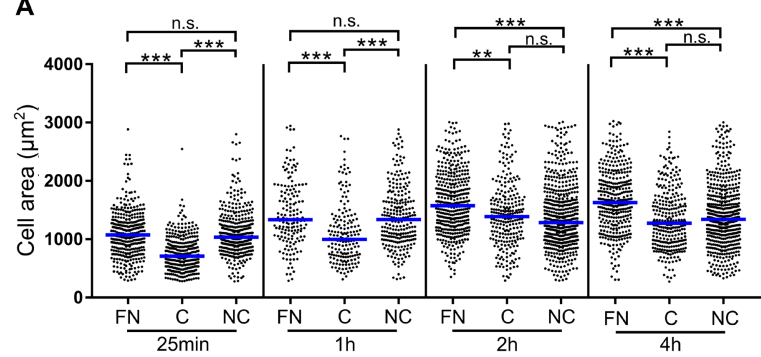

Figure 5. Cell morphology is dependent on the covalent state of RGD presentation. A) Cell area quantification reveals that cells seeded on the $\mathrm{COV}_{\mathrm{RGD}}$ (C) surfaces have diminished cell spreading compared to cells seeded on FN and NON_COV $V_{\text {RGD }}$ (NC) peptides. After 4h, cell area between $C_{\text {RGD }}$ and NON_COV $V_{\text {RGD }}$

B

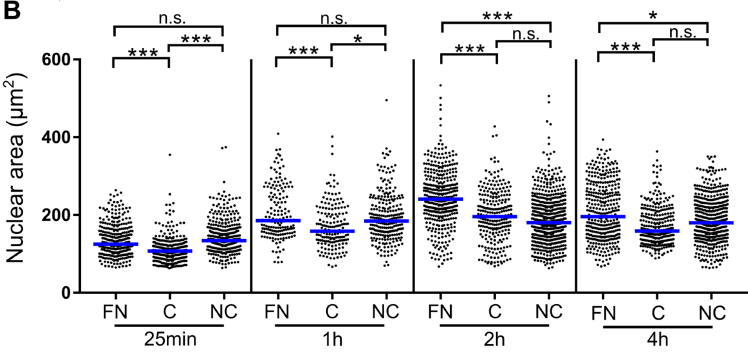

C



peptides becomes similar with the highest cell spreading observed on FN. B) Nuclear area follows a similar dynamics as cell area. C) Cell solidity quantification reveals that besides cell spreading also less protrusions are present on the $\mathrm{COV}_{\mathrm{RGD}}$ surface after 25 min. This effect remained present after 4h. Each dot represents the quantification of a single cell. The blue line represent median intensity levels of each condition. ( ${ }^{*} \mathrm{p}<0.05$; ${ }^{*} \mathrm{p}<0.01$;

Already during the early phase of cell attachment, we observed morphological differences between the cells cultured on the three surfaces. At $25 \mathrm{~min}$, cells grown on the COV RGD surface experienced a latency in cell spreading compared to cells attaching on either fibronectin or

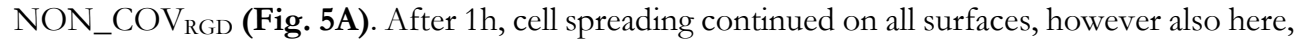
the $\mathrm{COV}_{\mathrm{RGD}}$ surface induced slower cell spreading. Only after two hours, cells grown on

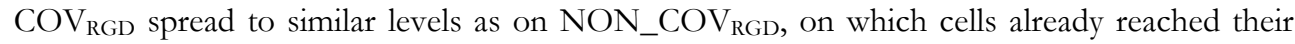
maximum spreading after $1 \mathrm{~h}$. Only for cells grown on FN, spreading continued to increase. Therefore, cells cultured on $\mathrm{FN}$ exhibit the largest spreading after $2 \mathrm{~h}$ and $4 \mathrm{~h}$ compared to the $\mathrm{COV}_{\mathrm{RGD}}$ and NON_COV $\mathrm{RGD}_{\mathrm{B}}$ surface. Nuclear spreading is known to correlate with cell spreading ${ }^{50}$, and also in our experimental setup, we observed that nuclear spreading coincided with differences in cell spreading elicited by the surfaces (Fig. 5B).

Besides cell spreading, other morphologic parameters can distinguish cells grown on the surfaces. Cell solidity represents the number of pixels located in the cell relative to the pixels in the convex hull, which is based on the area formed by drawing straight lines between the outmost cell boundaries (Fig. S3). This morphological parameter allows assessing protrusions levels of the cell. Of interest is that cell protrusions are already observable during the first $25 \mathrm{~min}$ of cell 


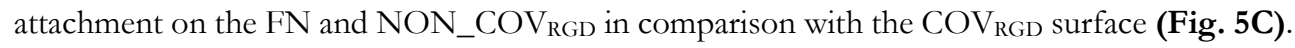
At $1 \mathrm{~h}$, solidity is sharply decreased for all surfaces, which can be interpreted with increased protrusion formation due to spreading. Cells cultured on $C_{\text {RGD }}$ kept exhibiting the highest solidity values compared to cells grown on FN and NON_COV to be due to decreased roundness and diminished protrusion formation. The FN surface induces slightly lower solidity levels compared to NON_COV RGD. These effects stabilize at $2 \mathrm{~h}$, with no


$\mathrm{COV}_{\mathrm{RGD}}$ being consistently highest (Fig. 5D). These observations confirm that the covalent state of RGD peptides profoundly changes focal adhesion characteristics and cell morphology.

\section{The (non-)covalent state of RGD peptides dictates the activation of early response transcription factors}

Because alterations in cell shape are known for inducing distinct gene expression patterns ${ }^{51}$, we investigated how the covalent state of RGD peptides influences the NIH3T3 fibroblast gene signature at these early time points. Due to the observations that RGD peptides exert their influence already at the early stages of cell-material contact, we applied microarray sequencing on RNA extracted from cells cultured on these surfaces for 30,60, and 120min. Already at $30 \mathrm{~min}$, differentially expressed genes (DEGs) on $\mathrm{COV}_{\mathrm{RGD}}$ and $\mathrm{NON}_{-} \mathrm{COV}_{\mathrm{RGD}}$ surfaces compared to FN are detectable (Fig. 6A). At 60min, NON_COV RGD surfaces induced 33 DEGs, and 19 DEGs were detected on the COV RGD surface compared to FN. Three DEGs are shared between the surfaces compared to $\mathrm{FN}$ at $30 \mathrm{~min}$, and eight DEGs at $60 \mathrm{~min}$. A common DEG between COV RGD and NON_COV RGD $_{\text {at }} 30 \mathrm{~min}$ is the transcription factor Fos with a fold change higher than two on both surfaces (Fig. 6B). Other early transcription factors become apparent after 60min with a fold change of 2.29 for Egr-1 on NON_COV RGD (Fig. 6C) and 1.37 on the $\mathrm{COV}_{\mathrm{RGD}}$ surface. Of interest, after $60 \mathrm{~min}$, only NON_COV $\mathrm{RGD}_{\text {D }}$ elicited significantly higher levels of Fos together with other early response transcriptional factors such as Egr-2 and JunB (Fig. 6D). These observations demonstrate that these surfaces elicit a distinct response in the early gene expression profiles of NIH3T3 fibroblasts.

Besides the expression of early response genes, we were interested in identifying pathway

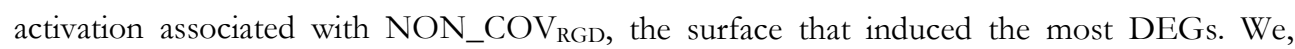
therefore, applied pathway analysis on the microarray data at $60 \mathrm{~min}$ to compare DEGs of

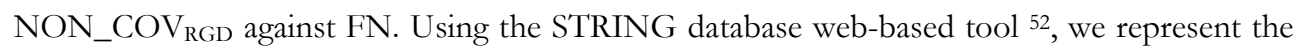
15 most significantly enriched gene ontology (GO) terms for "biological process", two GO terms for "cellular component", and seven KEGG pathways in Fig. 6E-G. For GO "biological process", we mainly find an upregulation for genes associated with metabolic activity, and responses to external stimuli (Fig. 6E). This wide variety in identified pathways is likely due to the association of early response genes with multiple cellular processes. For example, Egr-1 is activated through a plethora of stimulations like stress treatments ${ }^{53}$, viral infection ${ }^{54}$, growth factor signaling ${ }^{55,56}$, and mechanical stimulation ${ }^{57}$. Of which the latter is also known to acti vate Egr-2, Fos, and JunB ${ }^{57}$. In light of this, GO cellular compound analysis highlights the AP-1 
complex (Fig. 6F), which consists of both Fos and JunB ${ }^{58}$. Similar to Egr-1, AP-1 is implicated in a variety of biological processes, including proliferation ${ }^{59}$ and differentiation ${ }^{60}$.

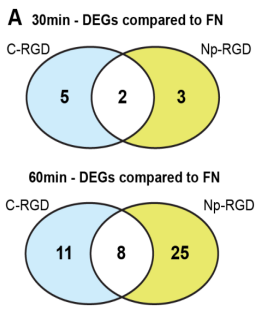

$E$

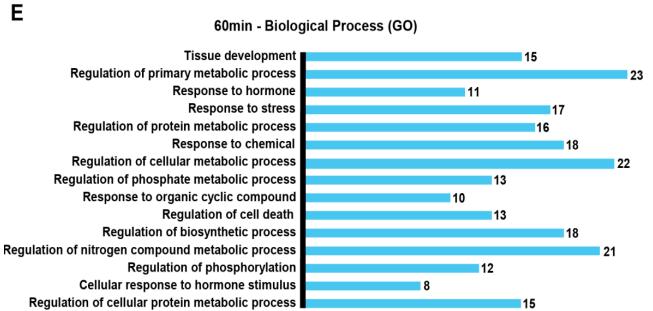

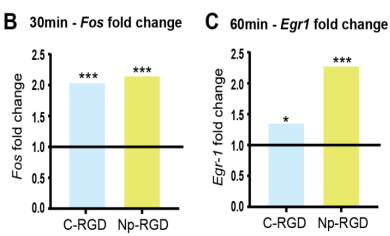

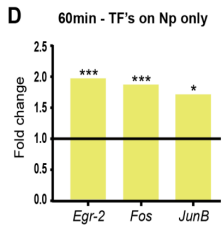

F



G

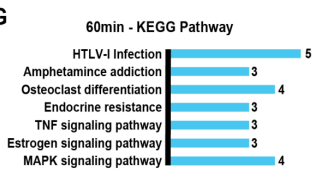

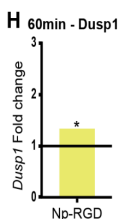

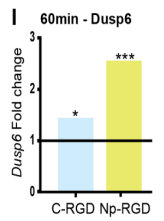

$J$

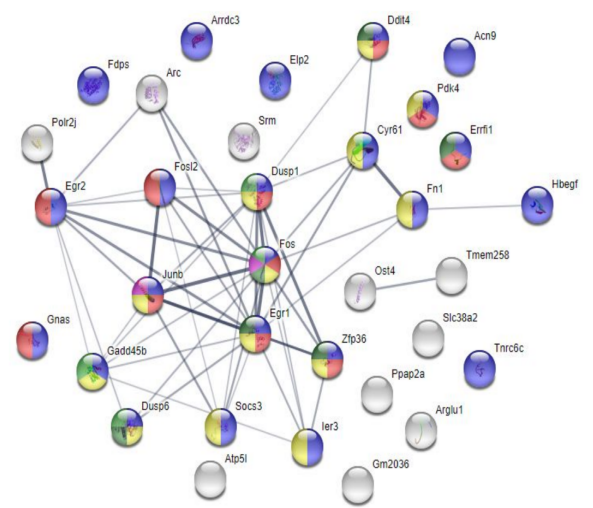

Primary Metabolic Process

Response to hormone

Regulation of cell death

Response to growth factor

AP-1 Complex

MAPK Signaling Pathway

Figure 6: Microarray study reveals a distinct gene expression signature in NIH3T3 fibroblasts. A) Distinct and common DEGs were identified both for COV $\mathrm{RGD}_{\mathrm{R}}$ surface and the NON_COV $V_{\text {RGD }}$ surface compared to FN. B) The transcription factor Fos is commonly upregulated on NON_COV RGD and the COV RGD surface. C) Also, the transcription factor Egr-1 is significantly upregulated on both $\mathrm{COV}_{\mathrm{RGD}}$ and $\mathrm{NON} \mathrm{COV}_{\mathrm{RGD}}$, with the highest elevation detected on the NON_COV $V_{\text {RGD }}$ surface. D) At 60 minutes, we see an upregulation of the transcription factors Egr-

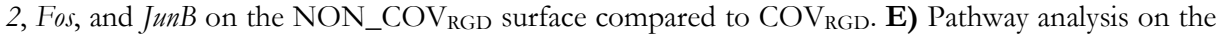

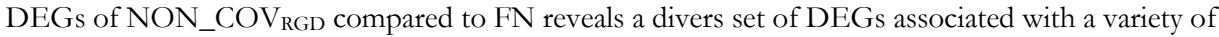
biological processes, including metabolic processes and cell signaling. F) GO pathway analysis focusing on cellular components reveals an association with the AP-1 transcription complex. G) KEGG pathway analysis of DEGs from NON_COV ${ }_{\text {RGD }}$ are associated with a divers set of pathways, including the MAPK signaling pathway. H) After 60 minutes, a slight upregulation is observed for

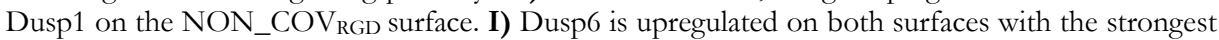

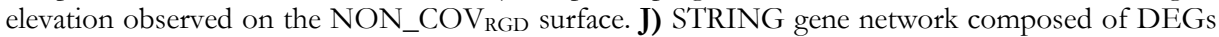
of NON_COV RGD compared to FN at 60 minutes reveals a gene hub of the key transcriptional factors Egr1, Fos, JunB which are associated with a variety of biological processes. $(* \mathrm{P}<0.05$; $* * \mathrm{P}<$ $0.01 ; * * * \mathrm{P}<0.001)$. 
KEGG pathway analysis highlights the involvement of these transcription factors in viral infection, addiction, differentiation, TNF, estrogen, and MAPK signaling (Fig. 6G). Furthermore, we found a slight elevation of Dusp1 expression on the NON_COV RGD surface

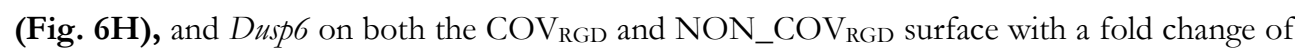
1.46 and 2.57 respectively (Fig. 6I). Both are involved in deactivating MAPK after a MAPK signaling event ${ }^{61}$. Visualization of the DEG network based on known protein-protein interactions indeed reveals a strong hub network around the early response transcription factors, which play a central role in both the GO and pathway results (Fig. 6J).

Next, we focused on gene expression differences between NIH3T3 fibroblasts grown on the $\mathrm{COV}_{\mathrm{RGD}}$ and NON_COV $\mathrm{RGD}_{\mathrm{R}}$ surfaces. For this, we further employed microarray studies after a $120 \mathrm{~min}$ culture on these surfaces, the time point after which morphological characteristics became stabilized. At $120 \mathrm{~min}$, gene expression differences become more profound between the


DEGs (Fig. 7A). Due to the higher number of DEGs, we applied ClueGO to cluster redundant biological terms in functionally grouped networks to improve biological interpretation ${ }^{62}$. This approach allowed the identification of several biological processes related to the apoptotic signaling pathway, immune system response, transcription, differentiation, reactive oxygen species, and growth factors (Fig. 7B). Again when using STRING, we see a cluster of transcription factors with high connectivity with each other and other proteins that are associated with these biological processes (Fig. 7C). Of interest, we could not detect JunB at this time point, which might be due to higher levels of Dusp1, Dusp6 observed at $60 \mathrm{~min}$, and Dusp4 at $120 \mathrm{~min}$. These genes contribute to the negative regulation of the MAPK pathway and junB. We also detected a higher presence of other transcription factors, including Klf2, Klf4, and Klf6, which are associated with a variety of biological processes, including differentiation and proliferation 63,64. Also, $B c l 6$ and $B c l 10$ as DEGs are present, which play a role in modulating immune signaling, preventing apoptosis, and the production of reactive oxygen species ${ }^{65-67}$. Of interest, we also observed an increase in $S r f$ known to be regulated by actin dynamics ${ }^{68}$, together with Egr-1 (Fig. 7D). $S r f$ is a crucial transcription factor and can bind to a multitude of gene targets ${ }^{69}$. $S r f$ can furthermore control the expression of the early transcription factors Fos, JunB, Egr-1, and is also auto-regulatory, depending on the binding with co-transcription factors ${ }^{69-71}$. These observations demonstrate a more robust mechanotransduction event elicited by the NON_COV RGD surface. 
A DEGs induced by NC-RGD compared to C-RGD

\section{B}


C

D
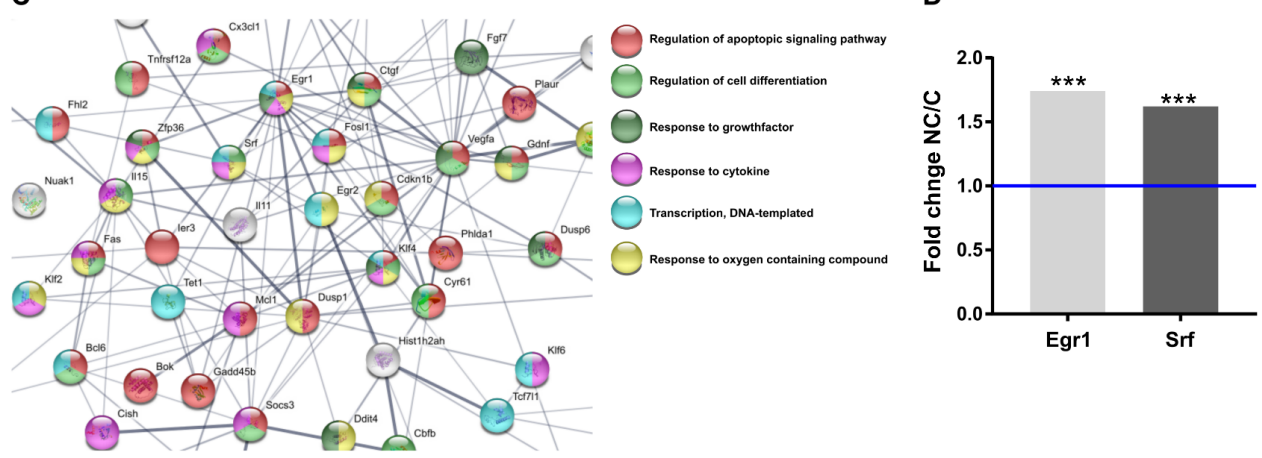


significant DEGs were upregulated and 69 were downregulated on the NON_COV RGD surface compared to $\mathrm{COV}_{\mathrm{RGD}}$. B) Pathway analysis reveals a large diversity in signaling pathways activation. C) Partial STRING representation of the DEGs color-coded with their association with GO biological pathways reveals a central interaction hub of transcription factors. D) Key transcription factors Egr-1 and Srf are upregulated on the NON_COV ${ }_{\text {RGD }}$ surface compared to COV $V_{\text {RGD }}$. 


\section{Non-Covalent RGD Surfaces elicit the Strongest Response in SRF, EGR-I, and F- actin Levels}

Due to the vital role of SRF and EGR-1 as mechanosensitive transcriptional factors, we further investigated their protein levels through immunostaining in NIH3T3 fibroblasts grown on FN,

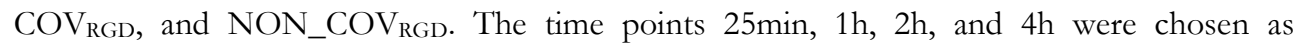
incubation times, after which cells were fixed and immunolabeled against either EGR-1 or SRF for protein quantification (Fig. 8A). Elevated EGR-1 levels were already detected after 25min for cells grown on both $\mathrm{COV}_{\mathrm{RGD}}$ and NON_COV $\mathrm{RGD}_{\mathrm{B}}$ compared to the FN surface (Fig. 8B). No significant difference was detected between $\mathrm{COV}_{\mathrm{RGD}}$ and NON_COV $\mathrm{RGD}_{\text {at }}$ at this time point. Similar to the microarray data, elevated EGR-1 levels were present for cells grown on both the $\mathrm{COV}_{\mathrm{RGD}}$ and NON_COV $\mathrm{RGD}_{\text {s }}$ surfaces at $1 \mathrm{~h}$ compared to FN. At $2 \mathrm{~h}$, protein levels further coincided with the microarray data, with EGR-1 levels on $\mathrm{COV}_{\mathrm{RGD}}$ dropping towards similar

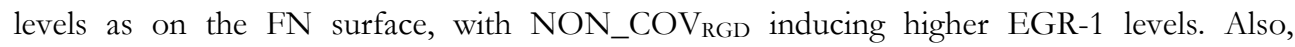
NON_COV RGD $_{\text {e }}$ elicited the most robust response in EGR-1 levels compared to COV RGD at $1 \mathrm{~h}$ and $2 \mathrm{~h}$. Of interest is that overall EGR-1 levels drop between 25min and $4 \mathrm{~h}$ on all surfaces, including $\mathrm{FN}$, indicating that the seeding procedure itself played a crucial role in eliciting higher EGR-1 levels. After 4h, only NON_COV RGD elevated EGR-1 levels compared to COV $\mathrm{RGD}_{\text {and }}$ FN. EGR-1 quantification on NON_COV $\mathrm{RGD}_{\text {is }}$ is characterized by single-cell heterogeneity, especially at $4 \mathrm{~h}$, with a subpopulation of NIH3T3 fibroblasts exhibiting high EGR-1 levels which contribute to the significant elevation towards the other surfaces.

SRF immunolabeling followed a similar trend with clear differences between the surfaces (Fig. 8C). Of interest is that already after $25 \mathrm{~min}$, both $\mathrm{COV}_{\mathrm{RGD}}$ and NON_COV $\mathrm{RGD}_{\text {display elevated }}$ nuclear SRF levels compared to FN, with the strongest response noticeable for NON_COV $\mathrm{RGD}_{\mathrm{B}}$ (Fig. 8D). After 25min, SRF levels dropped, again indicating an important role of cell seeding

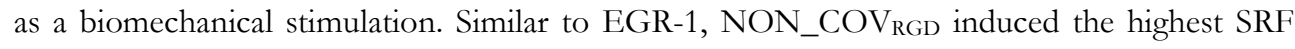
levels compared to both $\mathrm{COV}_{\mathrm{RGD}}$ and the FN surface on all time points. For cells grown on the $\mathrm{COV}_{\mathrm{RGD}}$ surface, higher levels were observed compared to the FN surface, yet reached equality at $4 \mathrm{~h}$.

Because of the relationship between actin dynamics and SRF ${ }^{68}$, we were interested in measuring F-actin levels across the different conditions to investigate similar trends. Visually, we found profound increased levels of F-actin for NIH3T3 fibroblasts grown on both the COV RGD and NON_COV $V_{\text {RGD }}$ surfaces after 4hours (Fig. 9A). F-actin quantification revealed a gradual increase of F-actin intensity levels on all surfaces that reached a peak at $2 \mathrm{~h}$ (Fig. 9B). After 4h, F-actin intensity levels dropped again. Across all time points, slight yet significant elevations of F-actin were observed for the NON_COV RGD $_{\text {surface compared to COV }}$ RD and FN surface.

In conclusion, here we unprecedentedly demonstrate that the (non-)covalent state of RGD presentation influences cell shape, F-actin structure, and coincides with an early gene and protein expression response in NIH3T3 cells. Here, non-covalent RGD presentation enhances cellular dynamics, induces faster focal adhesion maturation and more robust F-actin levels, as well as 
increasing the expression of transcription factors known to be involved in early growth response and actin signaling.

$\triangle$
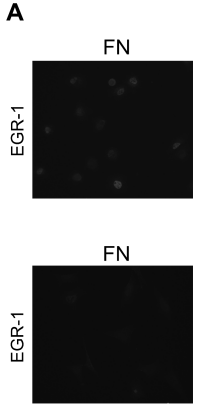

C

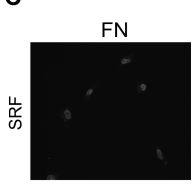

FN

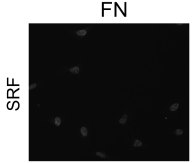

25 min - EGR-1

C

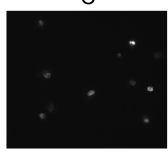

4h - EGR-1

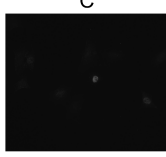

25 min - SRF



NC

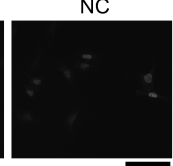

NC

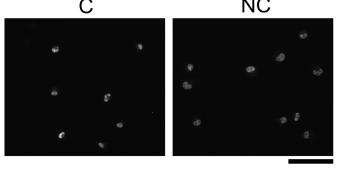

4h - SRF

C

NC
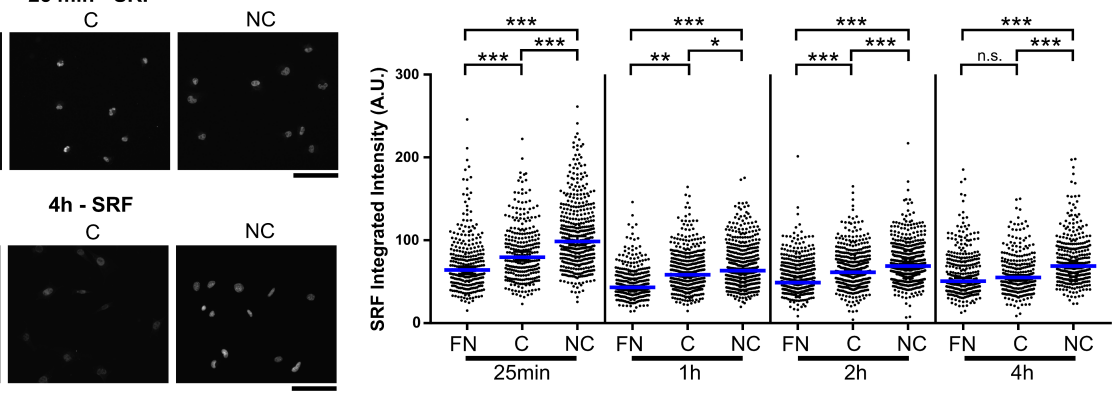

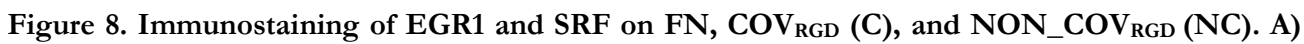
Elevated EGR-1 levels are present on both $\mathrm{C}$ and NON_COV RGD surfaces at $25 \mathrm{~min}$. This trend was still present at $4 \mathrm{~h}$, yet with slightly higher levels on the NON_COV ${ }_{\mathrm{RGD}}$ surfaces. B) Immunostaining quantification confirmed this observation with no statistical differences between $C_{\text {RGD }}$ and


EGR-1 levels dropped. After 4h, EGR-1 levels dropped on all surfaces while highest levels remained observed on the NON_COV RGD surface. C) Following a similar trend, the highest levels of SRF were

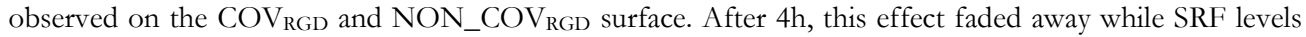
remaining highest on the $\mathrm{NON}_{-} \mathrm{COV}_{\mathrm{RGD}}$ surface. D) Immunostaining quantification confirmed this trend with NON_COV $V_{\text {RGD }}$ levels remaining significant highest across all conditions $(* \mathrm{p}<0.05 ; * * \mathrm{p}<0.01$; $* * *$ $\mathrm{p}<0.001)$. Each dot represents the quantification of a single cell. The blue line represent median intensity levels of each condition. $\left({ }^{*} \mathrm{p}<0.05 ;{ }^{* *} \mathrm{p}<0.01 ;{ }^{* * *} \mathrm{p}<0.001\right)$. 
A

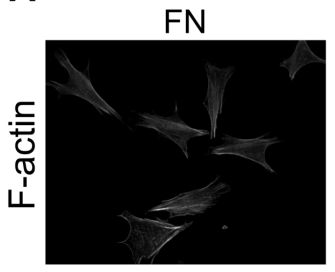

C-RGD

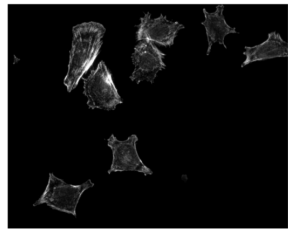

NC-RGD



B

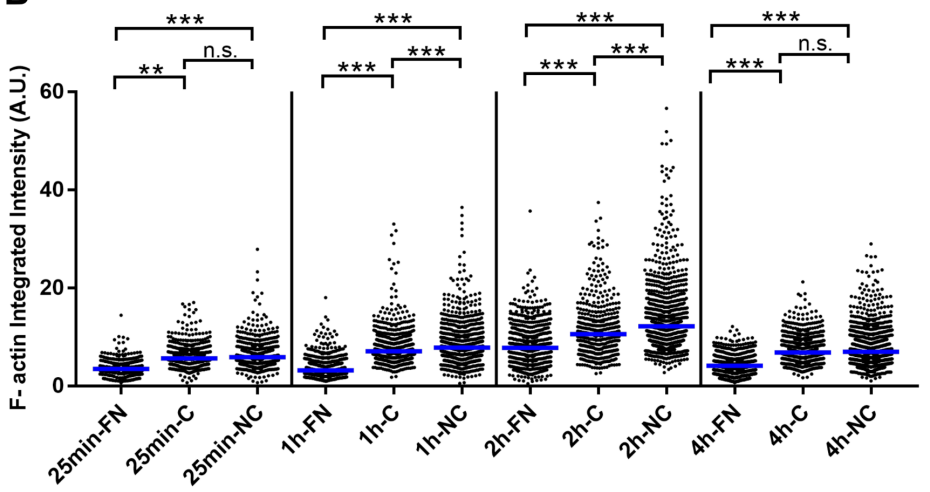

Figure 9: F-actin levels follow a similar pattern as the SRF and EGR-1 dynamics observed on the RGD surfaces. A) Visual observation of F-actin staining through phalloidin reveals increased intensity levels in NIH3T3 fibroblasts on the $\mathrm{COV}_{\mathrm{RGD}}$ and NON_COV $\mathrm{RGD}_{\mathrm{R}}$ surface after $2 \mathrm{~h}$. B) Immunofluorescent quantification indeed reveals that both $\mathrm{COV}_{\mathrm{RGD}}$ and NON_COV $\mathrm{RGD}_{\text {induce }}$

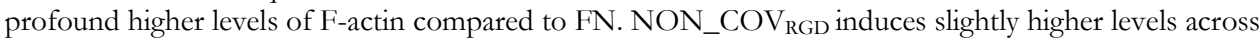
all conditions compared to the $\mathrm{COV}_{\mathrm{RGD}}$ surface. After $2 \mathrm{~h}$ of culture time, F-actin levels decrease on all conditions. Scale bar represent $100 \mu \mathrm{m}$. $\left(^{*} \mathrm{p}<0.05\right.$; ** $\mathrm{p}<0.01$; $\left.{ }^{* *} \mathrm{p}<0.001\right)$. Each dot represents the quantification of a single cell. The blue line represent median intensity levels of each condition. $\left({ }^{*} \mathrm{p}<0.05\right.$; ** $\left.\mathrm{p}<0.01 ;{ }^{* * *} \mathrm{p}<0.001\right)$.

\section{Discussion}

In this work, we move beyond classical matrix coatings and peptide imprints through presenting cells with dynamically active RGD peptides. How these peptides are modified in the culture dish can have different effects on cell behavior, as shown by studies where RGD spacing is altered ${ }^{72,73}$, where RGD organization follows ordered or disordered patterns ${ }^{74}$, or are presented as either a cyclic or linear RGD peptides ${ }^{75}$. Here, we further add to the design space RGD peptides that are either covalent or non-covalently presented and influence cell behavior. Non-covalent presented RGD peptides might reflect more physiologically relevant conditions compared to the standard covalent bound RGD peptides or coatings used in experimental setups. Therefore, these surfaces can be considered as a novel and valuable tool to guide cell behavior in the culture dish.

The effect of the non-covalent bound RGD peptides on the phenotype of the cell already becomes clear during the early stages of cell attachment. Focal adhesion maturation is more profound on these surfaces compared to covalently bound RGD peptides. This could indicate a 
stronger mechanical coupling between the cells and the surface. A multitude of integrins, part of the focal adhesion complex, are known to bind to these RGD sequences ${ }^{76}$, leading to cell adhesion, spreading, and other signaling events that influence proliferation and differentiation 77,78. Although we did not investigate the long-term consequences of these RGD peptides on the phenotype of the cells, exciting possibilities might arise with other cell types, such as for embryonic stem cells, of which is known that their phenotypic maintenance or differentiation potential can be altered depending on the matrix coating applied ${ }^{79}$. In this regard, replacing RGD with other signaling peptides found in matrix proteins such as collagen ${ }^{80}$ or laminin ${ }^{81}$ might further expand the design space of this platform.

The interaction of the NIH3T3 cells with the surface substrates offers novel insights into the elicited mechanobiology. Of interest, the type of peptide binding (fibronectin, covalent, or noncovalent) influences focal adhesion dynamics, cell shape, F-actin levels, and gene expression profiles. Since focal adhesions are protein complexes that are tightly connected with the cytoskeleton, it comes as no surprise that the different focal adhesion dynamics we observe on the surfaces coincide with different $\mathrm{F}$-actin architectures ${ }^{82}$. We believe the increased formation of focal adhesions and F-actin stress fibers on non-covalent RGD surfaces to be related to increased Rho activity, which affects the downstream activity of the transcription factor SRF, thereby given rise to its higher levels compared to other surfaces ${ }^{83,84}$. In this regard, it is interesting to mention that the elevated levels of SRF we quantified were highest at the early moments of cell attachment and before the most convincing F-actin and focal adhesion formation. There exist a tight relationship between altered actin dynamics and SRF activity ${ }^{68}$. In this experimental setup, it could be that SRF mediated the F-actin and focal adhesion formation since SRF is known to target a multitude of actin-related genes ${ }^{6,85}$. Additional research is, however, required to elucidate the exact mechanobiological mechanisms that are elicited by the surfaces. This platform can thereby be regarded as an exciting tool to study pathway signaling and dynamics associated with focal adhesion, actin, and SRF signaling.

Additional evidence for increased SRF activity on these surfaces is evident by the upregulation of FOS, known to be regulated by SRF through the presence of SRE elements in its promoter 86. Similarly, evidence exists that SRF is also involved in EGR1 regulation ${ }^{70}$. Both EGR1 and FOS are transcription factors associated with mechanobiological events such as stretching ${ }^{57}$ and control a wide variety of processes such as proliferation ${ }^{59}$ and differentiation ${ }^{60,87}$. It is, therefore, not surprising that these genes show a high interconnectedness with each other and other genes in the generated gene networks. In this study, we show that increased focal adhesion dynamics and F-actin formation coincides with higher levels of the transcription factors SRF, EGR1, and FOS, amongst others. Thereby clearly demonstrating that a non-covalent presentation of RGD peptides presentation has a profound impact on the mechanobiological response in these cells. In the future, Chip sequencing can elucidate the binding partners of these transcription factors, thereby allowing greater insights in the biomechanical consequences that this platform exerts. Furthermore, follow-up experiments with other cell types and long-term culturing can elucidate 
what other functional consequences are of applying non-covalent bound peptides in the culture environment.

\section{Materials and Methods}

\section{RGD Synthesis}

Thiol-functionalized methyl viologen (4, 4'-bipyridinium, 1-dodecyl-1'-methyl-, bromide iodide, MVSH) was synthesized and characterized as previously reported ${ }^{88}$. Peptides NpGGRGDSG

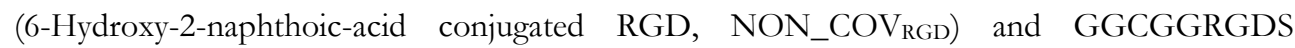
$\left(C_{\text {RGD }}\right.$ ) was synthesized using an automatic solid phase synthetic robot (Syro II, Multisyntech) following standard Fmoc procedures on a Rink amide resin. Purification was done by reversed phase high-performance liquid chromatography using water/acetonitrile gradient (10/90\% to 0/100\% with $0.1 \%$ Trifluoroacetic acid (TFA), RP-HPLC, C18 Column, Waters), followed by analysis with analytical HPLC and mass spectrometry.

\section{FT-IRRAS}

For the measurements, $200 \mathrm{~nm}$ gold coated Si wafers of sizes $2 \mathrm{x} 2 \mathrm{~cm}$ were functionalized with NON_COV $V_{\text {RGD }}$ and $\mathrm{COV}_{\mathrm{RGD}}$ as described above. Fourier Transform Infrared Reflection Absorption Spectroscopy (FT-IAS) was carried out using a Thermo Scientific TOM optical module. Polarized FT-IRRAS spectra were obtained by 1000 scans at $2 \mathrm{~cm}^{-1}$.

\section{Surface preparation for cell experiments}

$20 \mathrm{~nm}$ gold coated surfaces (Ssens, Enschede, Netherlands) were cleaned with piranha solution $\left(95 \% \mathrm{H}_{2} \mathrm{SO}_{4}\right.$ and $\left.30 \% \mathrm{H}_{2} \mathrm{O}_{2}(3: 1 \mathrm{v} / \mathrm{v})\right)$ for $15 \mathrm{~s}$, rinsed thoroughly in milliQ water and rinsed in absolute ethanol. Freshly cleaned substrates were incubated overnight (16-24h) in $1 \mathrm{mM}$ ethanolic solution of tetra(ethylene glycol) disulfide (HO-EG4-C11-S-S-C11-EG4-OH, referred as EG4-EG4, Prochimia) and maleimide-terminated mixed disulfide (HO-EG4-C11-S-S-C11EG6-OCH ${ }_{2}-\mathrm{CONH}$-maleimide, referred as EG4-maleimide, Prochimia) at defined molar ratios $(0.05 \%, 0.1 \%$ or $1 \%)$, protected under argon atmosphere. After overnight incubation, EG4Maleimide surfaces were thoroughly rinsed in absolute ethanol, were dried under $\mathrm{N}_{2}$ flow and further incubated and reacted with either freshly prepared $100 \mu \mathrm{M}$ MVSH in $0.1 \mathrm{Xphosphate}$ buffered saline (PBS) (first step for noncovalent RGD functionalization, referred as

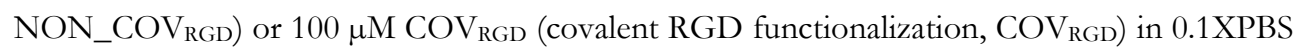
for $1 \mathrm{~h}$ at room temperature (RT). Subsequently surfaces were rinsed thoroughly in milliQ water, dried under $\mathrm{N}_{2}$ stream and $\mathrm{COV}_{\mathrm{RGD}}$ surfaces were stored under a nitrogen atmosphere before use. MVSH conjugated surfaces were further incubated with $50 \mu \mathrm{M}$ NON_COV $\mathrm{RGD}$


hydrate, Sigma) was dissolved in milliQ water at $80^{\circ} \mathrm{C}$ by sonication (for at least $30 \mathrm{~min}$ and then was brought to room temperature), and then a $50 \mu \mathrm{M} 0.1 \mathrm{XPBS}$ solution of $\mathrm{CB}[8]$ was prepared




MVSH surfaces that were functionalized with NON_COV $\mathrm{RGD}_{\mathrm{C}} \mathrm{CB}[8]$ were then gently and briefly rinsed with milliQ water. Next to RGD functionalized surfaces, as a control surface, piranha cleaned glass substrates (as above) were coated with fibronectin (FN, Sigma) at 10 $\mu \mathrm{g} / \mathrm{mL}$ (in $1 \mathrm{xPBS}$ ) for at least $1 \mathrm{~h}$ at room temperature, before cell seeding (see below). Surfaces were always used for cell experiments immediately after preparation.

\section{Cell culture}

NIH3T3 fibroblasts (ATCC ${ }^{\circledR}$ CRL-1658 ${ }^{\mathrm{TM}}$ ) were maintained in Dulbecco's Modified Eagle's Medium (DMEM, Sigma) supplemented with $2 \mathrm{mM} \mathrm{L-Glutamine} \mathrm{(Sigma),} 100 \mathrm{U} / \mathrm{mL}$ penicillin and $100 \mu \mathrm{g} / \mathrm{mL}$ streptomycin (Sigma) and 10\% v/v Fetal Bovine Serum (FBS, Sigma) and used between passage 15 and 30. Cells were sub-cultured on COV RGD, NON_COV RGD or FN surfaces at 10,000 cells $/ \mathrm{cm}^{2}$ in serum free basic medium (without FBS) for microarray analysis and gene expression profiling or for immunocytochemical analysis of cell adhesion, spreading, actin organization as well as protein expression analysis of microarray validation (also via immunocytochemistry).

\section{Immunocytochemical assays}

Cells on the surfaces were fixated with cold 4\% paraformaldehyde (PFA) for $10 \mathrm{~min}$ at room temperature (RT), rinsed in PBS (3X, 5 min each) left in PBS with 0.05\% sodium azide and kept at $4^{\circ} \mathrm{C}$ before immunocytochemical assays. For the experiments that are explained in Figures 1 3 and Figure S3 and S4, PFA fixed cells were permeabilized in 0.5\% Triton X-100 (Acros Organics) in PBS (Sigma) for $10 \mathrm{~min}$ at room temperature (RT). After permeabilization, cells were incubated with $0.1 \%$ Triton X-100 and 5\% (w/v) bovine serum albumin (BSA, Sigma) solution in PBS for $1 \mathrm{~h}$, to block nonspecific sites. Afterwards, cells were incubated with a solution of AlexaFluor-568 phalloidin (Molecular Probes, Thermo Fisher Scientific, 1:100) and monoclonal anti-human vinculin-FITC antibody (anti-hVIN-1, Sigma, 1:200) in $0.1 \%$ Triton-X100 and $5 \%(\mathrm{w} / \mathrm{v}) \mathrm{BSA}$ in PBS for $1 \mathrm{~h}$ at room temperature, in the dark. Cells were gently washed with PBS 3X, 10 min each. For characterization of cell polarization, after blocking non-specific sites, cells were incubated with, rabbit anti-Giantin antibody (Golgi marker, BioLegend, 1:400) in $0.1 \%$ Triton-X-100 and $5 \%(\mathrm{w} / \mathrm{v})$ BSA for $1 \mathrm{~h}$ at room temperature and cells were washed 2X, 0.1\% Triton-X-100 and 5\% (w/v) BSA, 10 min each. Then, cells were incubated with a solution of AlexaFluor-568 phalloidin (Molecular Probes, Thermo Fisher Scientific, 1:100) and secondary antibody (AlexaFluor-488 goat-anti-rabbit IgG, 1:400) in 0.1\% Triton-X-100 and 5\% $(\mathrm{w} / \mathrm{v}) \mathrm{BSA}$ in PBS for $1 \mathrm{~h}$ at room temperature, in the dark. Cells were washed $2 \mathrm{X}$ with $0.1 \%$ Triton-X-100 and 5\% (w/v) BSA in PBS and $1 \mathrm{X}$ with PBS. In both cases, nuclear staining was performed with DAPI nuclear stain in PBS (1:1000) for $10 \mathrm{~min}$ and after that cells were washed $2 \mathrm{X}$ with PBS (10 min each).

For the validation of microarray data, PFA fixed cells were permeabilized with $0.1 \%$ Triton-X100 in PBS for $10 \mathrm{~min}$, at RT. Then, cells were incubated with PBT solution $(0.02 \%$ Triton-X100 and $0.5 \%$ (v/v) goat serum (Sigma) in PBS) supplemented with $5 \%(\mathrm{v} / \mathrm{v})$ goat serum for $1 \mathrm{~h}$, 
to block nonspecific sites. Cells were then incubated with rabbit polyclonal anti-SRF antibody (G-20, Santa Cruz, 1:100) or rabbit monoclonal anti-EGR1 antibody (T.126.1, Thermo Fisher Scientific, 1:100) in PBT solution for $1 \mathrm{~h}$ at RT. After that cells were rinsed 3X in PBT, 10 min each. Cells were subsequently incubated with secondary antibody (AlexaFluor-488 goat-antirabbit IgG, 1:500) and AlexaFluor-568 phalloidin (1:60) for $1 \mathrm{~h}$ in PBT solution, and rinsed 3X in PBT, 10 min each. Nuclear staining was performed with DAPI (1:500) in PBT for 10 min. Cells were rinsed $1 \mathrm{X}$ with PBT and $1 \mathrm{X}$ with PBS, 10 min each and left in PBS. Finally surfaces were mounted with an aqueous mounting medium (Aqua Poly/Mount, Polysciences, Inc.) on coverslips and left to dry overnight at room temperature, in the dark. Then, cells were put at $4{ }^{\circ} \mathrm{C}$ and kept at dark before image acquisition.

\section{Image and Statistical Analysis}

For the experiments described in Figures 1-3, immunolabeled cells were imaged using an inverted fluorescence microscope with corresponding excitation and emission filters (Olympus, 1X71, Melville NY, USA). For shape analysis, 5-7 images taken per substrate condition. 2 independent experiments were done per condition. Fluorescence microscopy images of actin and DAPI were used for Cell Profiler based shape analysis.

For validation of microarray data and time point analysis of protein expression, at least 2 independent experiments were performed in duplicates per time point, validating surface preparation for each experiment based on the trends achieved in cellular morphology (or protein expression). Fluorescent images of the stained samples were acquired using an automated Nikon Eclipse Ti-U microscope with an Andor Zyla 5.5 4MP camera. For protein quantification and determining cell morphological characteristics, 10 random images of each sample were taken with a 20x objective (Nikon, NA=1.2). For detailed actin analysis, a 40x oil objective (Nikon, $\mathrm{NA}=1.3)$ was applied. Images were analyzed through CellProfiler 3.1.8 with a custom-made pipeline ${ }^{49}$. After illumination correction, nuclei morphology was captured using the Otsu adaptive thresholding method based on the DAPI staining. Subsequently, cell morphology was determined by applying Otsu adaptive thresholding and propagation algorithms based on the Phalloidin staining. Cells touching the border of the image were filtered out of the dataset. In addition, mislabeled cellular objects were identified by failure of the object to propagate in the Phalloidin channel and were subsequently removed. For quantification of F-Actin, SRF and EGR-1, the protein intensity values of each pixel inside the segmented nuclear or cellular area are summarized to calculate the integrated protein values of each individual cell.

Single cell parameter quantification from CellProfiler was further analyzed through $\mathrm{R}^{89}$, whereby image artifacts were removed from the data by applying arbitrary thresholds. PCA ${ }^{90}$ was performed in $\mathrm{R}$ through the function prcomp and visualized through the packages ggfortify ${ }^{91}$ and ggplot2 ${ }^{92}$.

GraphPad Prism 7.03 was applied for visualization of single cell protein quantification, morphological characteristics and determining statistical significance between the different 
conditions. Integrated protein intensities across different pertubations are considered statistical significant (P value lower then 0.05) after non-parametrical ANOVA test (Kruskal-Wallis).

\section{RNA Isolation}

After culturing cells on different surfaces (in triplicates), total RNA was isolated using the RNA isolation kit (Bioline, Isolate II). Then, from $190 \mathrm{ng}$ RNA, cRNA was synthesized using the Illumina ${ }^{\circledR}$ TotalPrep ${ }^{\mathrm{TM}}$ RNA amplification kit. Both RNA and cRNA quality were verified on a Bioanalyzer 2100 (Agilent).

\section{Microarray experiments and data analysis}

Microarrays were performed using Illumina HT-12 v4 expression Beadchips. Briefly, $750 \mathrm{ng}$ of cRNA was hybridized on the array overnight, after which the array was washed and blocked. Then, by addition of streptavidin $\mathrm{Cy}-3$, a fluorescent signal was developed. Arrays were scanned on an Illumina Beadarray reader and raw intensity values were background corrected in BeadStudio (Illumina). Further data processing and statistical testing were performed using $\mathrm{R}$ and Bioconductor statistical software. The probe-level raw intensity values were quantile normalized and transformed using variance stabilization (VSN). A linear modeling approach with empirical Bayesian methods, as implemented in Limma package was applied for differential expression analysis of the resulting probe-level expression values. $P$-values were corrected for multiple testing using the Benjamini and Hochberg method. Genes were considered differentially expressed when a corrected $p$-value below 0.05 was reached. The raw and normalized gene expression data and associated metadata have been uploaded in the Compendium for Biomaterial Transcriptomics.

To construct a gene network of the DEGs, we applied an online STRING analysis (https://string-db.org/). DEGs were further also included in the software ClueGo to cluster redundant biological terms in functionally grouped networks.

\section{Author Contributions}

t These authors contributed equally. J.B. and P.J. were involved in the conceptual development of this research. J.B. synthesized the NON_COV ${ }_{\text {RGD }}$ peptide and together with L.B.M.G. fabricated the surfaces and performed the study on the temporal evolution of cell spreading, focal adhesion development and cell polarization using fluorescence microscopy imaging with image analysis. J.B. fabricated the surfaces and performed the cell experiments for the microarray study, and together with G.K., isolated and amplified RNA, and together with J.v.d.P., performed the microarray experiments. J.v.d.P. and J.v.L. supervised the microarray equipment. D.H. analyzed the microarray data including pathway analysis. G.K. fabricated the surfaces and performed the cell experiments for microarray validation and time point analysis of SRF and EGR-1 expression, cell morphology, actin organization and inhibition experiments on NON_COV $V_{\text {RGD }}$ surfaces, performed immunocytochemistry for these experiments, was involved in the design of these experiments and prepared the figures. S.V. performed fluorescence 
microscopy imaging, image and data analysis for microarray validation and time point experiments, was involved in the design of these experiments and prepared the figures. A.V. was involved in image and data analysis. S.S. performed the expression and analysis of LifeAct GFP. E.C. synthesized and characterized the thiolated methylviologen and $C_{\text {RGD }}$ peptide. G.K., S.V., J.d.B. and P.J. wrote the manuscript.

\section{Acknowledgements}

The European Research Council is acknowledged for funding through Starters Grant 259183 (to P.J.) as well as P4.02 Superdices of the research program of the BioMedical Materials institute co-funded by the Dutch Ministry of Economic Affairs, Agriculture and Innovation (to P.J.) and Netherlands Organization for Scientific Research (NWO) through VIDI program 723.012.106 (to P.J.). J.d.B and S.V. acknowledge the financial support of the Dutch province of Limburg and the European Union's Horizon 2020 Program (H2020-MSCA-ITN-2015; Grant agreement 676338).

\section{References}

1. Campbell, I. D. \& Humphries, M. J. Integrin structure, activation, and interactions. Cold Spring Harb. Perspect. Biol. 3, 1-14 (2011).

2. Geiger, B., Spatz, J. P. \& Bershadsky, A. D. Environmental sensing through focal adhesions. Nat. Rev. Mol. Cell Biol. 10, 21-33 (2009).

3. Humphries, J. D. et al. Vinculin controls focal adhesion formation by direct interactions with talin and actin. J. Cell Biol. 179, 1043-1057 (2007).

4. Geiger, B. \& Yamada, K. M. Molecular architecture and function of matrix adhesions. Cold Spring Harb. Perspect. Biol. 3, 1-21 (2011).

5. $\quad$ Palecek, S. P., Loftus, J. C., Ginsberg, M. H., Lauffenburger, D. A. \& Horwitz, A. F. Integrinligand binding properties govern cell migration speed through cell-substratum adhesiveness. Nature 385, 537-540 (1997).

6. Olson, E. N. \& Nordheim, A. Linking actin dynamics and gene transcription to drive cellular motile functions. Nat. Rev. Mol. Cell Biol. 11, 353-65 (2010).

7. Parsons, J. T., Horwitz, A. R. \& Schwartz, M. A. Cell adhesion: Integrating cytoskeletal dynamics and cellular tension. Nat. Rev. Mol. Cell Biol. 11, 633-643 (2010).

8. Crowder, S. W., Leonardo, V., Whittaker, T., Papathanasiou, P. \& Stevens, M. M. Material Cues as Potent Regulators of Epigenetics and Stem Cell Function. Cell Stem Cell (2016). doi:10.1016/j.stem.2015.12.012

9. Leiss, M., Beckmann, K., Girós, A., Costell, M. \& Fässler, R. The role of integrin binding sites in fibronectin matrix assembly in vivo. Curr. Opin. Cell Biol. 20, 502-507 (2008).

10. Koçer, G. \& Jonkheijm, P. About Chemical Strategies to Fabricate Cell-Instructive Biointerfaces with Static and Dynamic Complexity. Advanced Healthcare Materials 7, 1-32 (2018).

11. Mas-Moruno, C. et al. $\alpha v \beta 3$ - or $\alpha 5 \beta 1$-Integrin-Selective Peptidomimetics for Surface Coating. Angewandte Chemie - International Edition 55, 7048-7067 (2016).

12. Karimi, F., O'Connor, A. J., Qiao, G. G. \& Heath, D. E. Integrin Clustering Matters: A Review of Biomaterials Functionalized with Multivalent Integrin-Binding Ligands to Improve Cell Adhesion, Migration, Differentiation, Angiogenesis, and Biomedical Device Integration. Adv. Healthc. Mater. 7, 128 (2018).

13. Kato, M. \& Mrksich, M. Using Model Substrates to Study the Dependence of Focal Adhesion Formation on the Affinity of Integrin-Ligand Complexes. Biochemistry 43, 2699-2707 (2004).

14. Kilian, K. A. \& Mrksich, M. Directing stem cell fate by controlling the affinity and density of ligand-receptor interactions at the biomaterials interface. Angew. Chemie - Int. Ed. 51, 4891-4895 (2012). 
15. Sobers, C. J., Wood, S. E. \& Mrksich, M. A gene expression-based comparison of cell adhesion to extracellular matrix and RGD-terminated monolayers. Biomaterials 52, 385-394 (2015).

16. Arnold, M. et al. Activation of integrin function by nanopatterned adhesive interfaces. ChemPhysChem 5, 383-388 (2004).

17. Cavalcanti-Adam, E. A. et al. Cell spreading and focal adhesion dynamics are regulated by spacing of integrin ligands. Biophys. J. 92, 2964-2974 (2007).

18. Arnold, M. et al. Induction of cell polarization and migration by a gradient of nanoscale variations in adhesive ligand spacing. Nano Lett. 8, 2063-2069 (2008).

19. Liu, Y. et al. Nanoparticle tension probes patterned at the nanoscale: Impact of integrin clustering on force transmission. Nano Lett. 14, 5539-5546 (2014).

20. Pallarola, D. et al. Interface immobilization chemistry of cRGD-based peptides regulates integrin mediated cell adhesion. Adv. Funct. Mater. 24, 943-956 (2014).

21. Gooding, J. J., Parker, S. G., Lu, Y. \& Gaus, K. Molecularly engineered surfaces for cell biology: From static to dynamic surfaces. Langmuir 30, 3290-3302 (2014).

22. Diaz, C., Neubauer, S., Rechenmacher, F., Kessler, H. \& Missirlis, D. Recruitment of $\alpha \nu \beta 3$ integrin to $\alpha 5 \beta 1$ integrin-induced clusters enables focal adhesion maturation and cell spreading. J. Cell Sci. $133,(2020)$.

23. Frith, J. E., Mills, R. J. \& Cooper-White, J. J. Lateral spacing of adhesion peptides influences human mesenchymal stem cell behaviour. J. Cell Sci. 125, 317-327 (2012).

24. Valdembri, D. \& Serini, G. Regulation of adhesion site dynamics by integrin traffic. Curr. Opin. Cell Biol. 24, 582-591 (2012).

25. Ng, C. C. A. et al. Using an electrical potential to reversibly switch surfaces between two states for dynamically controlling cell adhesion. Angew. Chemie - Int. Ed. 51, 7706-7710 (2012).

26. Salierno, M. J., García, A. J. \& Del Campo, A. Photo-activatable surfaces for cell migration assays. Adv. Funct. Mater. 23, 5974-5980 (2013).

27. Kadem, L. F. et al. Rapid Reversible Photoswitching of Integrin-Mediated Adhesion at the Single-Cell Level. Adv. Mater. 28, 1799-1802 (2016).

28. Lamb, B. M. \& Yousaf, M. N. Redox-switchable surface for controlling peptide structure. J. Am. Chem. Soc. 133, 8870-8873 (2011).

29. Luo, W. \& Yousaf, M. N. Tissue morphing control on dynamic gradient surfaces. J. Am. Chem. Soc. 133, 10780-10783 (2011).

30. Liu, D., Xie, Y., Shao, H. \& Jiang, X. Using azobenzene-embedded self-assembled monolayers to photochemically control cell adhesion reversibly. Angew. Chemie - Int. Ed. 48, 4406-4408 (2009).

31. Farrukh, A. et al. Photoactivatable Adhesive Ligands for Light-Guided Neuronal Growth. ChemBioChem 19, 1271-1279 (2018).

32. Goor, O. J. G. M., Hendrikse, S. I. S., Dankers, P. Y. W. \& Meijer, E. W. From supramolecular polymers to multi-component biomaterials. Chem. Soc. Rev. 46, 6621-6637 (2017).

33. Brinkmann, J. et al. About supramolecular systems for dynamically probing cells. Chem. Soc. Rev. 43, 4449-4469 (2014).

34. An, Q. et al. A supramolecular system for the electrochemically controlled release of cells. Angew. Chemie - Int. Ed. 51, 12233-12237 (2012).

35. Freeman, R. et al. Instructing cells with programmable peptide DNA hybrids. Nat. Commun. 8, (2017).

36. Sankaran, S., Jaatinen, L., Brinkmann, J., Zambelli, T. \& Jonkheijm, P. Cell Adhesion on Dynamic Supramolecular Surfaces Probed by Fluid Force Microscopy-Based Single-Cell Force Spectroscopy. ACS Nano 11, 3867-3874 (2017).

37. Uhlenheuer, D. A., Petkau, K. \& Brunsveld, L. Combining supramolecular chemistry with biology. Chem. Soc. Rev. 39, 2817-2826 (2010).

38. Boekhoven, J. \& Stupp, S. I. 25th anniversary article: Supramolecular materials for regenerative medicine. Adv. Mater. 26, 1642-1659 (2014).

39. Neirynck, P. et al. Supramolecular control of cell adhesion via ferrocene-cucurbit[7]uril hostguest binding on gold surfaces. Chem. Commun. 49, 3679-3681 (2013).

40. Ren, T. et al. Dual Responsive Surfaces Based on Host-Guest Interaction for Dynamic Mediation of Cell-Substrate Interaction and Cell Migration. Adv. Mater. Interfaces 4, 6-11 (2017). 
41. Boekhoven, J., Pørez, C. M. R., Sur, S., Worthy, A. \& Stupp, S. I. Dynamic Display of Bioactivity through Host - Guest Chemistry. Angew. Chemie - Int. Ed. 52, 12077-12080 (2013).

42. Voskuhl, J., Sankaran, S. \& Jonkheijm, P. Optical control over bioactive ligands at supramolecular surfaces. Chem. Commun. 50, 15144-15147 (2014).

43. Barth, A. Infrared spectroscopy of proteins. Biochim. Biophys. Acta - Bioenerg. 1767, 1073 1101 (2007).

44. Le Saux, G. et al. Spacing of integrin ligands influences signal transduction in endothelial cells. Biophys. J. 101, 764-773 (2011).

45. Lawson, C. et al. FAK promotes recruitment of talin to nascent adhesions to control cell motility. J. Cell Biol. 196, 223-232 (2012).

46. Gauthier, N. C., Masters, T. A. \& Sheetz, M. P. Mechanical feedback between membrane tension and dynamics. Trends Cell Biol. 22, 527-535 (2012).

47. Singhvi, R. et al. Engineering Cell Shape and Function. Science (80-. ). 264, 696-698 (1994).

48. McBeath, R., Pirone, D. M., Nelson, C. M., Bhadriraju, K. \& Chen, C. S. Cell shape, cytoskeletal tension, and RhoA regulate stem cell lineage commitment. Dev. Cell 6, 483-495 (2004).

49. McQuin, C. et al. CellProfiler 3.0: Next-generation image processing for biology. PLoS Biol. 16, $1-17$ (2018).

50. Vishavkarma, R. et al. Role of actin filaments in correlating nuclear shape and cell spreading. PLoS One 9, (2014).

51. Kilian, K. A., Bugarija, B., Lahn, B. T. \& Mrksich, M. Geometric cues for directing the differentiation of mesenchymal stem cells. Proc. Natl. Acad. Sci. 107, 4872-4877 (2010).

52. Szklarczyk, D. et al. STRING v10: protein-protein interaction networks, integrated over the tree of life. Nucleic Acids Res. 43, D447-52 (2015).

53. Lim, C. P., Jain, N. \& Cao, X. Stress-induced immediate-early gene, egr-1, involves activation of p38/JNK1. Oncogene 16, 2915-2926 (1998).

54. Li, S.-W. et al. SARS coronavirus papain-like protease induces Egr-1-dependent up-regulation of TGF- $\beta 1$ via ROS/p38 MAPK/STAT3 pathway. Sci. Rep. 6, 25754 (2016).

55. Gregg, J. \& Fraizer, G. Transcriptional Regulation of EGR1 by EGF and the ERK Signaling Pathway in Prostate Cancer Cells. Genes and Cancer 2, 900-909 (2011).

56. Bhattacharyya, S. et al. Smad-independent transforming growth factor- $\beta$ regulation of early growth response-1 and sustained expression in fibrosis: Implications for scleroderma. Am. J. Pathol. 173, 1085-1099 (2008).

57. Eliasson, P., Andersson, T., Hammerman, M. \& Aspenberg, P. Primary gene response to mechanical loading in healing rat Achilles tendons. J. Appl. Physiol. 114, 1519-1526 (2013).

58. Rauscher, F. J., Voulalas, P. J., Franza, B. R. \& Curran, T. Fos and Jun bind cooperatively to the AP-1 site: reconstitution in vitro. Genes Dev. 2, 1687-1699 (1988).

59. Güller, M. et al. C-Fos overexpression increases the proliferation of human hepatocytes by stabilizing nuclear Cyclin D1. World J. Gastroenterol. 14, 6339-6346 (2008).

60. Mehic, D., Bakiri, L., Ghannadan, M., Wagner, E. F. \& Tschachler, E. Fos and Jun proteins are specifically expressed during differentiation of human keratinocytes. J. Invest. Dermatol. 124, 212-220 (2005).

61. Owens, D. M. \& Keyse, S. M. Differential regulation of MAP kinase signalling by dual-specificity protein phosphatases. Oncogene 26, 3203-3213 (2007).

62. Bindea, G. et al. ClueGO: A Cytoscape plug-in to decipher functionally grouped gene ontology and pathway annotation networks. Bioinformatics 25, 1091-1093 (2009).

63. Pearson, R., Fleetwood, J., Eaton, S., Crossley, M. \& Bao, S. Krüppel-like transcription factors: A functional family. Int. J. Biochem. Cell Biol. 40, 1996-2001 (2008).

64. Nemer, M. \& Horb, M. E. The KLF family of transcriptional regulators in cardiomyocyte proliferation and differentiation. Cell Cycle 6, 117-121 (2007).

65. Kumagai, T. et al. The proto-oncogene Bcl6 inhibits apoptotic cell death in differentiationinduced mouse myogenic cells. Oncogene 18, 467-475 (1999).

66. Kurosu, T., Fukuda, T., Miki, T. \& Miura, O. BCL6 overexpression prevents increase in reactive oxygen species and inhibits apoptosis induced by chemotherapeutic reagents in B-cell lymphoma cells. Oncogene 22, 4459-4468 (2003). 
67. Zhou, H. et al. Bcl10 activates the NF- $x$ B pathway through ubiquitination of NEMO. Nature 427, 167-171 (2004).

68. Sotiropoulos, A., Gineitis, D., Copeland, J. W. \& Treisman, R. Signal-regulated activation of serum response factor is mediated by changes in actin dynamics. Cell 98, 159-169 (1999).

69. Selvaraj, A. \& Prywes, R. Expression profiling of serum inducible genes identifies subset of SRF target genes that are MKL dependent. BMC Mol. Biol. 5, 1-15 (2004).

70. Schratt, G. et al. Serum response factor is required for immediate-early gene activation yet is dispensable for proliferation of embryonic stem cells. Mol. Cell. Biol. 21, 2933 (2001).

71. Gualdrini, F. et al. SRF Co-factors Control the Balance between Cell Proliferation and Contractility. Mol. Cell 64, 1048-1061 (2016).

72. Massia, S. P. An RGD spacing of $440 \mathrm{~nm}$ is sufficient for integrin alpha V beta 3- mediated fibroblast spreading and $140 \mathrm{~nm}$ for focal contact and stress fiber formation. J. Cell Biol. 114, 1089-1100 (2004).

73. Kuen, Y. L. et al. Nanoscale adhesion ligand organization regulates osteoblast proliferation and differentiation. Nano Lett. 4, 1501-1506 (2004).

74. Huang, J. et al. Impact of Order and Disorder in RGD Nanopatterns on Cell Adhesion. Nano Lett. 9, 1111-1116 (2009).

75. Kämmerer, P. et al. Immobilisation of linear and cyclic RGD-peptides on titanium surfaces and their impact on endothelial cell adhesion and proliferation. Eur. Cells Mater. 21, 364-372 (2016).

76. $\quad$ Plow, E. F., Haas, T. A., Zhang, L., Loftus, J. \& Smith, J. W. Ligand binding to integrins. J. Biol. Chem. 275, 21785-21788 (2000).

77. Abdul Kafi, M., El-Said, W. A., Kim, T. H. \& Choi, J. W. Cell adhesion, spreading, and proliferation on surface functionalized with RGD nanopillar arrays. Biomaterials 33, 731-739 (2012).

78. Kantlehner, M. et al. Surface coating with cyclic RGD peptides stimulates osteoblast adhesion and proliferation as well as bone formation. Angew. Chemie (International Ed. English) 39, 107-114 (2000).

79. Hayashi, Y. et al. Integrins Regulate Mouse Embryonic Stem Cell Self-Renewal. Stem Cells 25, 3005-3015 (2007).

80. Kim, J. K. et al. A novel binding site in collagen type III for integrins $\alpha 1 \beta 1$ and $\alpha 2 \beta 1$. J. Biol. Chem. 280, 32512-32520 (2005).

81. Tomaselli, K. J., Damsky, C. H. \& Reichardt, L. F. Interactions of a neuronal cell line (PC12) with laminin, collagen IV, and fibronectin: Identification of integrin-related glycoproteins involved in attachment and process outgrowth. J. Cell Biol. 105, 2347-2358 (1987).

82. Ciobanasu, C., Faivre, B. \& Le Clainche, C. Actin dynamics associated with focal adhesions. Int. J. Cell Biol. (2012). doi:10.1155/2012/941292

83. Hill, C. S., Wynne, J. \& Treisman, R. The Rho family GTPases RhoA, Rac1, and CDC42Hs regulate transcriptional activation by SRF. Cell 81, 1159-1170 (1995).

84. Liu, H. W. et al. The RhoA/Rho kinase pathway regulates nuclear localization of serum response factor. Am. J. Respir. Cell Mol. Biol. 29, 39-47 (2003).

85. Miano, J. M., Long, X. \& Fujiwara, K. Serum response factor: Master regulator of the actin cytoskeleton and contractile apparatus. Am. J. Physiol. - Cell Physiol. 292, 70-81 (2007).

86. Treisman, R. Identification of a Protein-Binding Site That Mediates Transcriptional Response of the c-fos Gene to Serum Factors. Cell 46, 567-574 (1986).

87. Guerquin, M. J. et al. Transcription factor EGR1 directs tendon differentiation and promotes tendon repair. J. Clin. Invest. 123, 3564-3576 (2013).

88. Stoffelen, C., Voskuhl, J., Jonkheijm, P. \& Huskens, J. Angewandte Dual Stimuli-Responsive Self-Assembled Supramolecular Nanoparticles. Angew. Commun. 53, 3400-3404 (2014).

89. R Core Team. R: A language and environment for statistical computing. R Found. Stat. Comput. 90. Wold, S., Esbensen, K. I. M. \& Geladi, P. Principal Component Analysis. Chemom. Intell. Lab. Syst. 2, 37-52 (1987).

91. Tang, Y., Horikoshi, M. \& Li, W. ggfortify: Unified Interface to Visualize Statistical Result of Popular R Packages. R J. 8.2, 478-489 (2016).

92. Wickham, H. ggplot2: Elegant Graphics for Data Analysis. Springer-Verlag New York (2016). 


\section{Supplementary Figures}
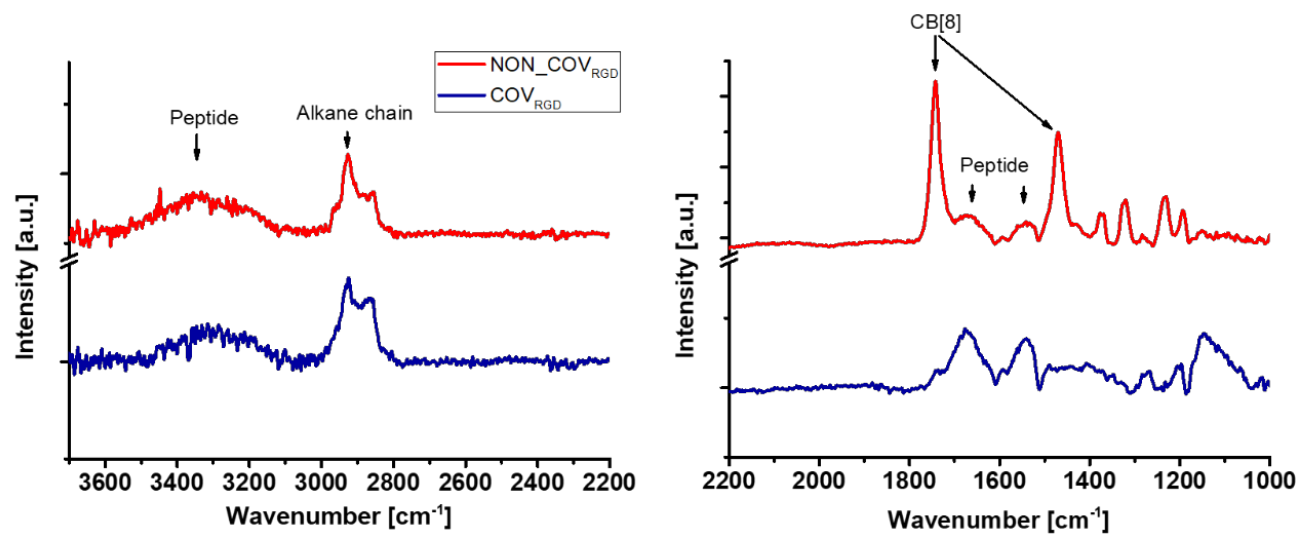

Figure S1: Monolayer validation through FT-IRRAS. Illustrated are FT-IRRAS spectra of COV RGD (blue) and NON_COV RGD (red). CB [8] peaks at $1477 \mathrm{~cm}^{-1}$ and $1740 \mathrm{~cm}^{-1}$ and RGD peptide at $3300 \mathrm{~cm}^{-1}$.

A

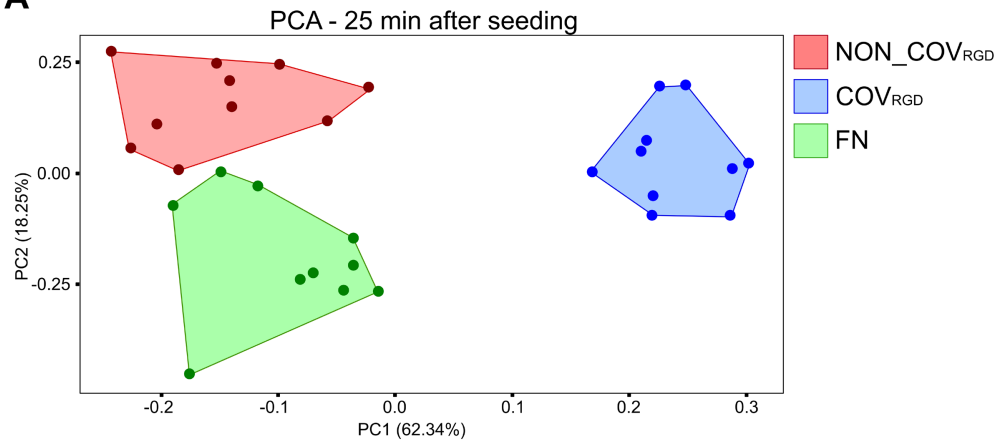

B

PCA - 4h after seeding



Figure S2: PCA analysis based on morphological parameters distinguishes cells grown on the different surfaces. A) Already after $25 \mathrm{~min}$, NIH3T3 fibroblasts can be classified in distinct clusters. B) Also after $4 \mathrm{~h}$ of culture on the surfaces, morphological characteristics of the NIH3T3 fibroblasts were different between the surfaces. 

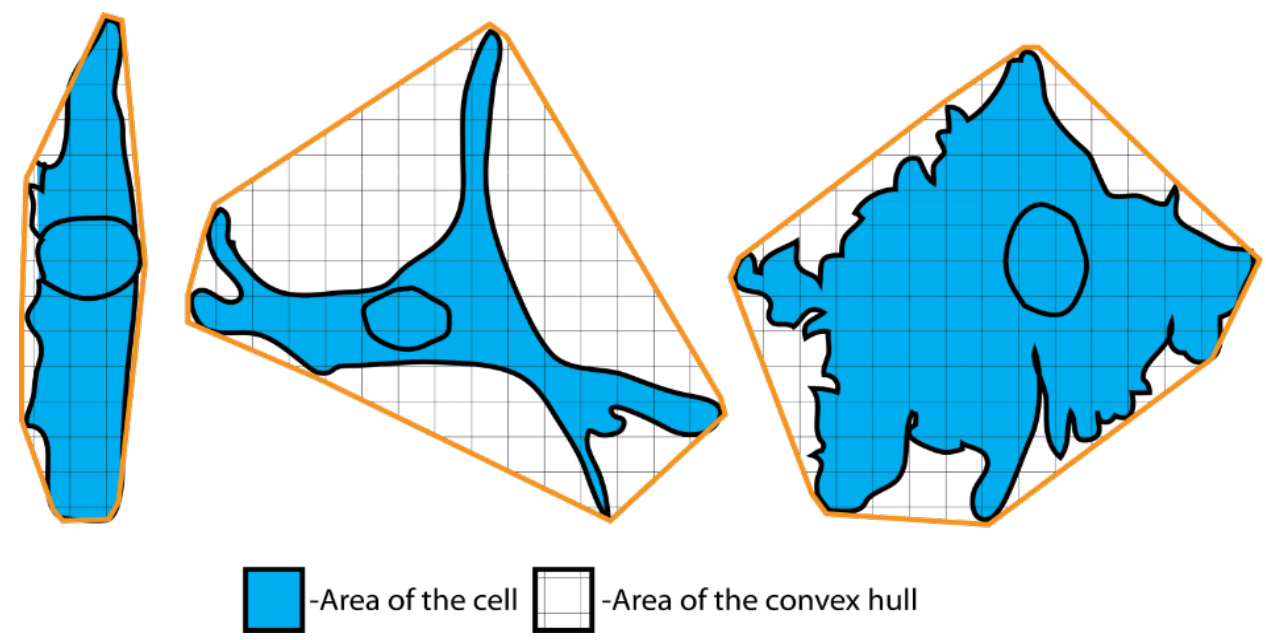

-Area of the cell $\square$-Area of the convex hull

Figure S3: Illustration of solidity, a Cell Profiler morphological parameter. Solidity is defined as the proportion of the pixels in the convex hull that are also in the region. Solidity levels are low for cells that have a branched morphology. 


\section{Chapter VII}

\section{Epigenetic Regulation of \\ Mesenchymal Stem Cells by Micro-}

\section{Topographies}

Steven Vermeulen ${ }^{1,2}$, Bart Van Puyvelde ${ }^{3}$, Laura Bengtsson del Barrio ${ }^{1}$, Ben van der Veer ${ }^{1}$, Maarten Dhaenens ${ }^{3}$, Jan de Boer ${ }^{2}$

${ }^{1}$ Laboratory for Cell Biology-Inspired Tissue Engineering, MERLN Institute, University of Maastricht, Maastricht, The Netherlands

${ }^{2}$ BioInterface Science Group, Department of Biomedical Engineering and Institute for Complex Molecular Systems, University of Eindhoven, Eindhoven, The Netherlands.

${ }^{3}$ Laboratory of Pharmaceutical Biotechnology, Department of Pharmaceutics, Ghent University, Belgium

Manuscript in preparation 


\section{Abstract}

Physical structures are known for their ability to control cell and nuclear morphology. Since the shape of the nucleus regulates chromatin architecture, gene expression, and cell identity, surface structures can play an essential role in controlling cell phenotype. In this study, we explore how micro-topographies influence nuclear morphology, their effect on histone modifications, and the expression on histone-associated proteins through histone mass spectrometry and microarray analysis. We noticed that nuclear confinement is associated with a loss of both histone acetylation and nucleoli abundance. Pathway analysis revealed a substantial reduction in gene expression associated with chromosome organization and the cell cycle. We connect these findings with a quiescent-like phenotype in mesenchymal stem cells, as shown by the retainment of multipotency on micro-topographies after long-term culture conditions. Furthermore, the influence of micro-topographies on nuclear morphology and proliferation was dynamic in origin, as shown by a re-adaptation on the flat surface after topographical culture conditions. These findings provide novel insights on how biophysical signaling influences nuclear organization and subsequent cell phenotype. 


\section{Introduction}

Primary cells exposed to the in vitro culture environment can quickly lose morphological characteristics and phenotypic marker expression, such as is the case for chondrocytes and tenocytes ${ }^{1,2}$. This phenomenon also occurs for multipotent cells, such as mesenchymal stem cells (MSCs), leading to a gradual decrease of multipotency after multiple passages ${ }^{3}$. This problematic nature of the standard culture environment impedes tissue-engineering applications since tissue or organ construction requires a significant number of cells. However, different approaches exist to alleviate this problematic phenomenon. The addition of growth factors can improve multipotency in MSCs ${ }^{4}$. Coating the surface with matrix compounds can alter the differentiation of embryonic stem cells or the phenotypic maintenance of primary cells 5 . Furthermore, changing physical properties such as elasticity can improve phenotypic maintenance of stem cells ${ }^{6}$, or enhance their differentiation ${ }^{7}$. An interesting and further extension of biomaterial properties capable of directing cell fate are physical cues, which have gained popularity in recent years for cell culture applications.

Physical cues can mimic essential aspects of the physiological environment. For example, scaffolds or gels mimic 3D environments, which results in signaling events otherwise absent on a regular flat surface ${ }^{8}$. Physical cues change morphologic characteristics, which leads to profound alterations in cell phenotype. In this context, the use of adhesive islands can profoundly alter cell area and morphology, leading to altered differentiation events in MSCs 9,10. Similar changes in morphology and phenotype are induced by surface structures such as grooves ${ }^{11}$ and topographies ${ }^{12}$. The biological pathways elicited by these structures are usually associated with cytoskeleton dynamics. An important consideration is that physical cues also have a direct effect on nuclear shape and resulting phenotype. Distinguishing if physical cues exert their effect either through cell or nuclear morphology changes are challenging to assess due to the strong relationship between the two parameters ${ }^{13,14}$. Nevertheless, nuclear shape alterations are associated with epigenetic changes, such as chromatin organization or histone modifications, which result in altered gene expression ${ }^{15,16}$. Therefore, utilizing physical cues such as microtopographies are an excellent tool for elucidating how modifying nuclear shape effects epigenetic and the resulting functional consequences.

\section{Results}

\section{Micro-topographies elicit a broad nuclear confinement and deformation diversity in MSCs}

It is well established that physical cues alter cellular morphological characteristics $10,17,18,19$, including those elicited by topographical structures 20-22. However, despite the strong relationship between cell and nuclear shape, the effect of physical cues on nuclear architecture has not yet been thoroughly investigated. We, therefore, aimed to investigate how different physical cues in micro-topographical dimensions affect nuclear morphology. For this, we utilized 
the TopoChip, of which 2176 different surface topographies were created in silico by using three primitives in unique combinations (Fig. 1A). Before seeding MSCs on the TopoChips, we assessed the quality of the substrate using profilometric imaging. This allowed confirmation of the spatial dimensions of the topographical features and the walls separating the individual units (Fig. 1B). After a positive quality assessment, MSCs were seeded on eight TopoChips and cultured for 48h, after which F-actin and DNA were fluorescently labeled (Fig. 1C). Since the TopoChip platform contains four flat surfaces and each micro-topography in duplicate, a total of 32 flat surfaces and 16 replicas of the same micro-topography were included in the analysis. High-content imaging of each unit enabled the identification of 41,540 cells. We observed a broad cellular morphological heterogeneity between the individual units, similar to previous TopoChip studies involving tenocytes ${ }^{2}$ and MSCs ${ }^{23}$. Furthermore, we found a diversity of nuclear morphologies as well, with varying degrees of nuclear area and deformation (Fig. 1D).

A

A Feature construction

B



Figure 1: Micro-topographies create a large variety in cell- and nuclear morphological characteristics. A) Through a circle, rectangle, and triangle, we created a large variety in surface design. B) Profilometric image of the TopoChip platform. A $2 \times 2 \mathrm{~cm}^{2}$ chip with 2176 unique micro-topographies with a height of 10 $\mu \mathrm{m}$, and walls of $30 \mu \mathrm{m}$. C) Illustration of

C
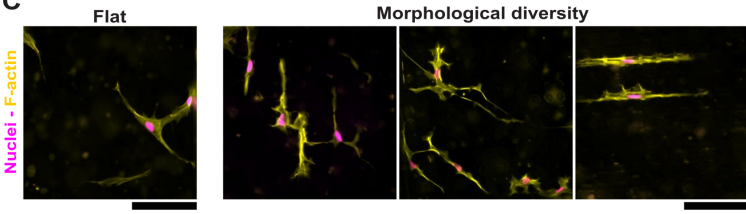

D

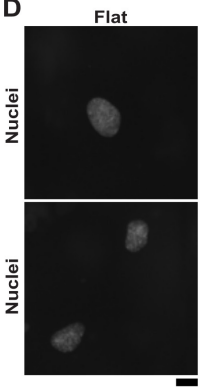

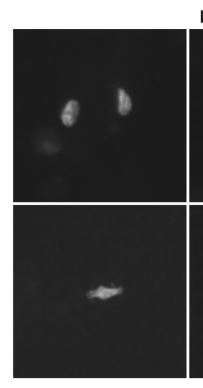

Nuclear diversity

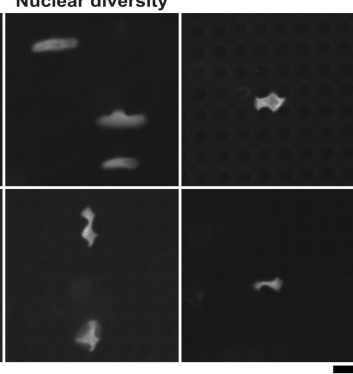
morphological diversity elicited by microtopographies. Depicted are MSCs cultured on the flat control surface and three microtopographical surfaces. F-actin was stained with Phalloidin conjugated with an Alexa Fluor. The nucleus was counterstained with Hoechst33258. Scale bar represents $100 \mu \mathrm{m}$. D) Representative images of nuclear deformation occurring on microtopographies. Depicted are nuclei of MSCs cultured on the flat control surface and a random sample of MSCs cultured on micro-topographies. In general, microtopographies elicit smaller nuclei, which different degrees of elongation and branching. Scale bar represents $10 \mu \mathrm{m}$.

Next, we quantified this diversity by measuring nuclear morphological characteristics through CellProfiler ${ }^{24}$. We provide the reader in Supplementary Fig. 1 a visual explanation of the morphological parameters. Both cell and nuclear area were decreased for the majority of MSCs grown on the micro-topographies, with 984 of 2176 micro-topographies (45\%) reaching significant lower cell area (Fig. 2A) and 580 of 2176 micro-topographies (26\%) inducing lower nuclear area (Fig. 2B). Plotting nuclear and cell areas indeed revealed a correlation between these two parameters (Supplementary Fig. 2), a known phenomenon also observed on other 
platforms such as for adhesive islands ${ }^{14}$. Also, we found through quantifying the morphological parameters compactness and eccentricity, a global trend for micro-topographies to induce nuclear elongation. Here, we determined that 1692 micro-topographies (77\%) induced significantly higher compactness levels, while 1670 micro-topographies (76\%) induced significantly higher eccentricity levels compared to a flat surface (Fig. 2C-D). Besides elongation, we were interested in determining nuclear branching or deformation through measuring solidity levels. Here, we determined that 982 micro-topographies (45\%) induced significantlylower solidity levels and thus induced nuclear deformation (Fig. 2E). These findings demonstrate that micro-topographical diversity elicits diversity in nuclear morphology.

Next, we characterized the relationship between the feature parameters of the microtopographies and morphological outcomes. A complete overview of the surface parameters such as pattern area, the number of primitives, or feature area, is documented in Supplementary Table 1. We employed random forest algorithms to create models that associate nuclear morphological parameters with the feature descriptors. We used $75 \%$ of the lowest 200 and $75 \%$ of the highest 200 scoring TopoUnits associated with inducing a morphological characteristic. The accuracy of the model was assessed on the remaining 25\%. Models were trained with 10fold cross-validation. When assessing which feature characteristics induced either low or high nuclear area, we found that pattern area and wavenumber 0.1 (WN0.1), a Fourier transformation algorithm, were important determinants for the prediction model, with an area under the curve (AUC) of 94\% and a 93\% accuracy (Supplementary Fig. 3). Plotting the WN0.1 and pattern area of micro-topographies inducing the smallest and largest nuclear area indeed allows a separation between the top and lowest 200 micro-topographies (Fig. 2F). Of interest is the existence of a subpopulation of cells with a sizeable nuclear area induced by a large pattern area and an intermediate WN0.1. This is an indication of very dense patterns where cells have little room to settle between the structures. The same parameters were also significant determinants for influencing compactness (Fig. 2G) and solidity levels (Fig. 2H). Of interest here, is that WN0.1 can achieve this separation, independent of pattern area, indicating that the distance between the features is essential to achieve elongation and deformation of the nuclei. 

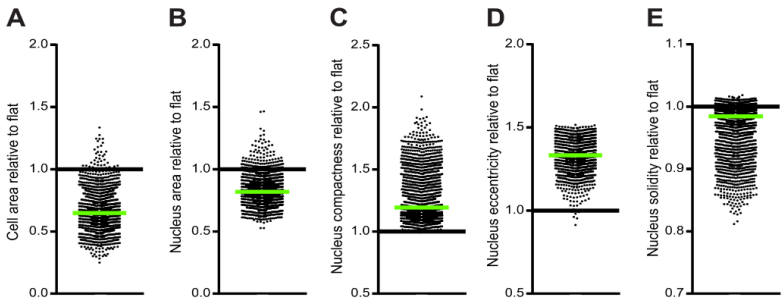

F

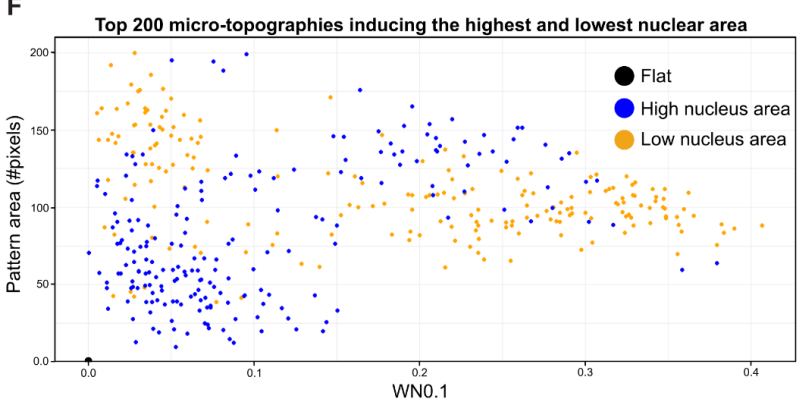

G Top 200 micro-topographies inducing the highest and lowest nuclear compactness


Figure 2: Pattern area and wavenumber 0.1 promote nuclear deformation. A-E) Microtopographies induce a spectrum of different cell and nuclear sizes, elongation (compactness and eccentricity), and branching (solidity), with flat residing on the far side of the spectra. Each dot represents the median quantified values of an individual micro-topography. The black line represents the flat surface, and the green line represents the median of all individual values. F) A large pattern area generally promotes a smaller nuclear size. An exception was found with surface patterns exhibiting a high pattern area, and intermediate WN0.1, characteristic of high-density patterns. G-H) Increased elongation (increased compactness), and branching (lower solidity), was found on micro-topographies with intermediate pattern areas and high WN0.1, characteristic for surfaces that allow spacing in between the micro-topographies. 


\section{Nuclear confinement is associated with reduced histone 3 and histone 4 acetylation}

After determining that micro-topographies influence nuclear morphology, we wanted to investigate how this affects the histone landscape. For this, we selected the PS-1018 surface, which we can fabricate in a $100 \mathrm{~mm}$ dish format, allowing sufficient cell numbers for downstream experiments. This surface induces elongated morphology in MSCs and a substantial reduction in nuclear area (Supplementary Figure 4). We further employed histone massspectrometry that allowed us to profile histone modifications on both the histone $3(\mathrm{H} 3)$ and histone 4 (H4) tail. $48 \mathrm{~h}$ after cell seeding, we performed mass-spectrometry on the chromatin fraction and were able to detect ten histone modifications on the $\mathrm{H} 3$ tail (Fig. 3A), and six histone modifications on the $\mathrm{H} 4$ tail (Fig. 3B). On the $\mathrm{H} 3$ tail, we found an increase in $\mathrm{H} 3 \mathrm{~K} 9 \mathrm{Me}$, a decrease in $\mathrm{H} 3 \mathrm{~K} 9 \mathrm{Me} 2$ and $\mathrm{H} 3 \mathrm{~K} 27 \mathrm{me} 2$, with no observed difference in $\mathrm{H} 3 \mathrm{~K} 9 \mathrm{Me} 3$ between flat and PS-1018. These observations are associated with alterations in gene transcription speed ${ }^{25,26}$. The majority of significant differences were measured on the $\mathrm{H} 4$ tail, where acetylation levels were decreased for H4K2, H4K5, H4K8, and H4K12, indicative for chromatin compaction ${ }^{27}$. For H4K2, we also observed higher dimethylation levels. Besides a $48 \mathrm{~h}$ time point, we also quantified histone modifications at a $4 \mathrm{~h}$ and $24 \mathrm{~h}$ time point. When quantifying the total amount of $\mathrm{H} 4$ acetylation, we noticed that the decreased levels already becomes apparent $4 \mathrm{~h}$ after cell seeding (Fig. 3C). Of interest, after $48 \mathrm{~h}$, we noticed an increase in global H4 acetylation both on the flat and PS-1018 surface. Nevertheless, throughout all time points, H4 acetylation remained lowest on the PS-1018 surface.

Due to the observed decrease in global H4 acetylation levels, we were interested in exploring if this effect was also apparent for the $\mathrm{H} 3$ tail since not all acetylation sites were detected with histone mass-spectrometry. For this, we employed immunostaining with antibodies targeting global acetylation on both the $\mathrm{H} 3$ and $\mathrm{H} 4$ tail, while targeting $\mathrm{H} 3 \mathrm{~K} 9 \mathrm{Me} 2$ as extra validation of the mass-spectrometry data (Fig. 3D). Through immunostaining, we found a decrease in $\mathrm{H} 3$ acetylation intensity levels when MSC are cultured on the PS-1018 surface (Fig. 3E). A similar observation was apparent for $\mathrm{H} 4$ acetylation levels, of which a more substantial decrease was observed compared to $\mathrm{H} 3$ acetylation (Fig. 3F). For $\mathrm{H} 3 \mathrm{~K} 9 \mathrm{Me} 2$ we found a slight decrease in intensity levels (Fig. 3G). Of interest is the heterogeneous $\mathrm{H} 3 \mathrm{~K} 9 \mathrm{Me} 2$ intensity levels between MSCs cultured on the PS-1018 surface. Furthermore, we observed that some MSCs exhibit high H3K9Me2 intensity levels at the nuclear periphery through a heat map visualization (Supplementary Figure 5), which could indicate altered interaction dynamics between chromatin and the nuclear periphery ${ }^{28}$. In general, this data associates the nuclear deformation that micro-topographies elicit most substantially with reduced histone acetylation levels. 


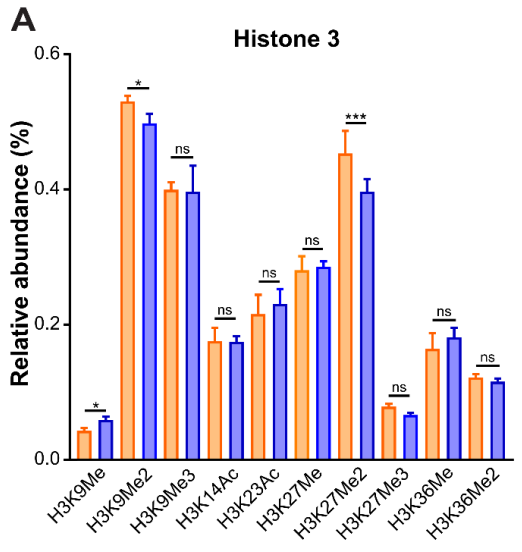

D

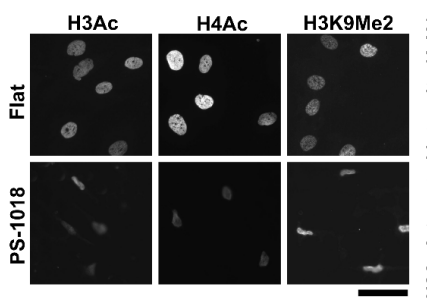

B



Histone 4

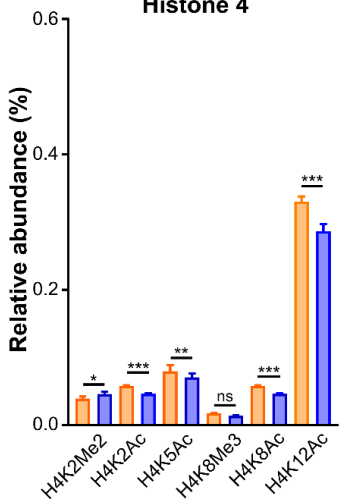

$\mathbf{F}$

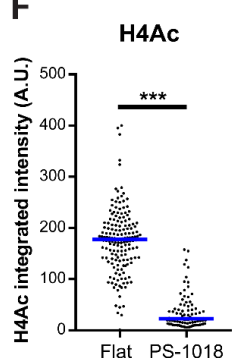

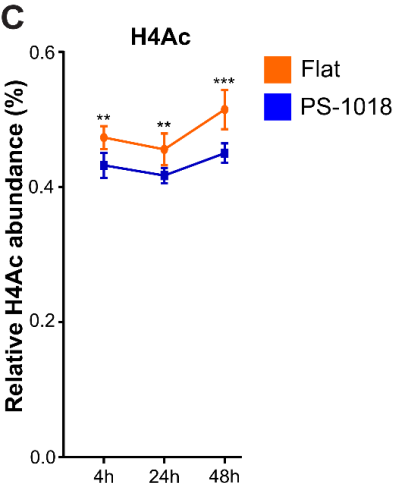



Figure 3: Histone mass-spectrometry and immunostaining reveal a decline in histone acetylation for MSCs cultured on the micro-topography PS-1018. A) Histone mass-spectrometry performed on $\mathrm{H} 3$ reveals an $\mathrm{H} 3 \mathrm{~K} 9 \mathrm{Me} / \mathrm{Me} 2$ ratio increase and a decrease in $\mathrm{H} 3 \mathrm{~K} 27 \mathrm{Me} 2$ when $\mathrm{AD}$ hMSCs are cultured on the PS-1018 surface after 48h. B) Histone mass-spectrometry performed on H4 reveals a general decrease in acetylation levels, with a slight increase in H4K2 dimethylation. Bar plot represents mean with error bars representing SD. C) Global $\mathrm{H} 4$ acetylation levels are already decreased 4h after seeding on the PS-1018 surface. Acetylation levels slightly increase after 48h for MSCs cultured on both the flat and PS-1018 surface compared to the $4 \mathrm{~h}$ and $24 \mathrm{~h}$ time point. D) Immunolabeled images of global H3/H4 acetylation, and H3K9Me2 of MSCs cultured on flat and PS-1018 after 48h. Scale bar represents $50 \mu \mathrm{m}$. E) Immunolabeling quantification reveals a general decrease in $\mathrm{H} 3$ acetylation levels. F) Immunolabeling quantification reveals a general decrease in $\mathrm{H} 4$ acetylation levels. G) Immunolabeling quantification reveals a decrease in $\mathrm{H} 3 \mathrm{~K} 9 \mathrm{Me} 2$ levels. Each dot represents individual nuclei. The blue line indicates the median. $(* \mathrm{P}<0.05 ; * * \mathrm{P}<0.01 ; * * * \mathrm{P}<0.001)$.

\section{Micro-topographical diversity can lead to specific levels of histone 3 acetylation}

Since different surface feature parameters correlate to nuclear morphology, and microtopographies decrease acetylation levels, we were interested in the correlation between nuclear shape and histone acetylation. We selected six different topographies, seeded MSCs on them, and immunolabeled $\mathrm{H} 3$ acetylation after 48 hours of culture. Intensity level quantification demonstrated a general decrease in $\mathrm{H} 3$ acetylation (Figure 4A), similar to the PS-1018 surface. Of interest is that different levels of acetylation were observed in MSCs on different topographies. We also included the compounds trichostatin A (TSA), and valproic acid (VPA) as a reference, both HDAC inhibitors known to increase histone acetylation levels ${ }^{29,30}$. These 
compounds indeed increased acetylation levels in MSCs compared to a flat surface. The design of these surfaces is found in Figure 4B, where we see that surface PS-463 exhibits a low pattern area, with PS-2113, PS-340, PS-990 exhibiting intermediate pattern areas, with high-density patterns apparent on surface PS-818 and PS-1476. Visual inspection of the H3 acetylation staining reveals that nuclear area is strongly influenced through this patterned area, as shown before with the Topochip screen (Figure 4C).

A

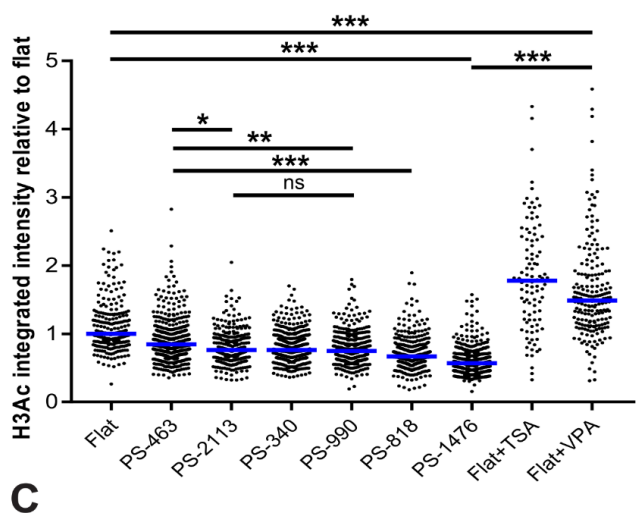

B

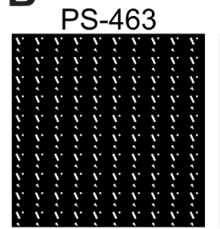

PS-990



PS-2113



PS-818

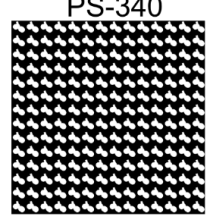

PS-1476

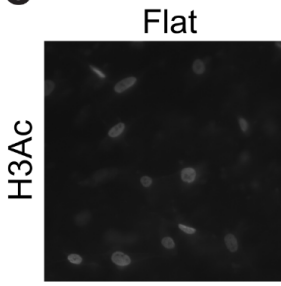

PS-463
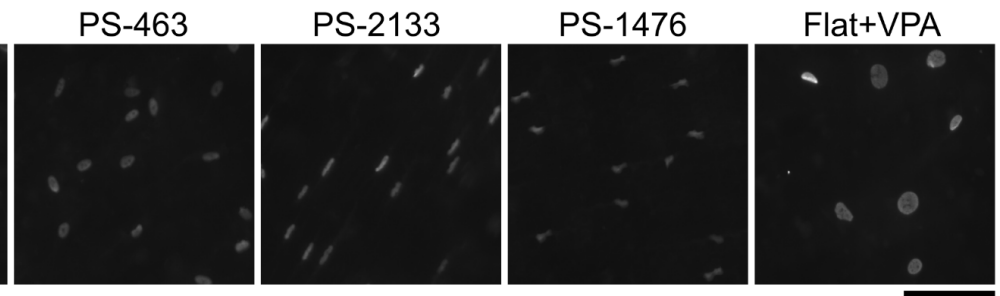

Figure 4: H3 acetylation levels can be differentially regulated by micro-topographies. A) Quantification of immunolabeled $\mathrm{H} 3$ acetylation on six different micro-topographies reveals different levels of histone acetylation in MSCs. Surface PS-463 elicits the least difference in acetylation levels compared to a flat surface, while PS-1476 elicits the strongest reduction. No significant difference was observed between PS-2133, PS-340, and PS-PS-990. HDAC inhibitors TSA and VPA induce elevated acetylation levels $*$ P $<0.05$; ** $\mathrm{P}<0.01 ; \mathrm{P}<0.001)$. Each dot represents individual nuclei. The blue line represents the median. B) In silico design of the micro-topographies used in this screen. PS-463 contains patterns with a low pattern area. PS-1476 contains patterns with a high pattern area. C) MSCs cultured on PS-463 exhibit smaller nuclei compared to MSCs on flat, yet with the same round morphology. Nuclei of MSCs grown on PS-2133 exhibit smaller, and elongated morphology with PS-1476 inducing the smallest and deformed nuclei. Scale bar represents $50 \mu \mathrm{m}$. 


\section{Micro-topographies induce chromatin condensation and a loss of nucleoli}

\section{structure}

After assessing that micro-topographies induce nuclear confinement, which coincides with profound reductions in global acetylation levels, we were interested in determining how other nuclear structures are affected. Foremost, we visualized chromatin condensation through creating a heat map of the Hoechst33258 signal on the PS-1018 surface (Fig. 5A). We found increased chromatin condensation levels as to be expected with the lower acetylation levels we observed ${ }^{31}$. Next, we wanted to investigate how micro-topographies influence the number of nucleoli. For this, we stained the nucleoli with nucleophosmin (NPM1), which plays a function in essential biological processes such as ribosome biogenesis ${ }^{32}$, mRNA processing ${ }^{33}$, and chromatin remodeling ${ }^{34}$. We also stained fibrillarin (FBL), a ribonucleoprotein involved in prerRNA processing and ribosome assembly ${ }^{35}$, and nucleolin (NCL), a nucleolar protein which is also associated with pre-rRNA processing ${ }^{36}$ and chromatin condensation ${ }^{37}$. Of interest, when comparing the immunostainings between flat and surface PS-1018, we determined a reduction in nucleoli through all immunostainings (Fig. 5B-D). For NPM1, also a more diffuse presence in the nucleus with a slight translocation towards the cytoplasm was present. These findings indicate sharp alterations in nucleolar function, which warrants further investigation, and is to our knowledge the first observation made in the context of biomaterials.

A

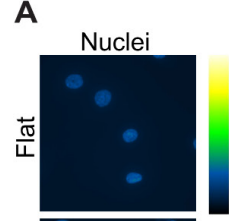

B


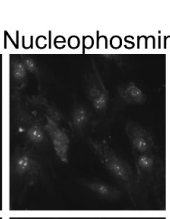

C
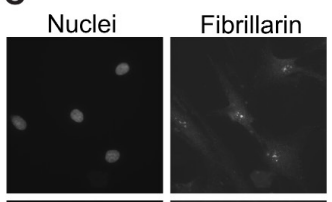

D


Figure 5: Micro-topographies induces chromatin condensation and a reduction of nucleoli. A) Heat map representation of Hoechst 33258 intensity levels reveals that the PS-1018 surface induces chromatin condensation. B-D) Immunostaining of the nucleoli markers nucleophosmin, fibrillarin, and nucleolin reveal a decrease in the number of nucleoli present when MSCs were cultured on the PS-1018 surface. Nuclei counterstained with Hoechst. Scale bar represents $50 \mu \mathrm{m}$.

\section{Micro-topographies induce a distinct proteomic and transcriptomic profile that influences chromosome organization}

We analyzed through mass-spectrometry differences in the presence of the proteins between MSCs cultured on flat and the PS-1018 surface. In total, we detected 221 proteins with a significant 1.3 fold change. We first examined how many proteins were associated with the GO term "Structural constituent of the ribosome" to extend the previous observation concerning the reduced presence of nucleolus-associated proteins. We found a higher expression of 20 ribosomal proteins in MSCs cultures on PS-1018 compared to flat and one ribosomal protein with a lower presence (Fig. 6A). An overview of these proteins is found in Supplementary 160 
Table 2. This is an interesting finding considering that the alterations in nucleoli-associated proteins might disrupt rRNA processing and ribosome assembly. This might indicate an increased synthesis of ribosomal proteins to compensate for the reduction in ribosomal assembly in the nucleoli. However, since we also report a general decrease in protein synthesis for MSCs grown on micro-topographies in another study (manuscript submitted), which is associated with reduced ribosomal assembly, we believe this to be related to ribosomal protein functions independent from the ribosome ${ }^{38}$. Next, we searched our microarray data library of MSCs cultured on micro-topographies for additional information if these proteins are also regulated at the transcriptional level. For this, we selected microarray data from surface PS-281, which exhibit similarities with the PS-1018 surface concerning morphological characteristics such as nuclear deformation and elongation (Supplementary Figure 6). Here, we report a downregulation of five, and the upregulation of one ribosomal protein, indicating that the control of ribosomal protein synthesis might be differentially regulated between the transcriptomic and proteomic level (Figure 6B).

Next, we assessed how micro-topographies influence proteins involved in chromosome organization. Of interest, we found in the proteomics data six proteins exhibiting a lower presence and ten proteins exhibiting a higher presence on the PS-1018 surface compared to flat associated with the GO term "chromosome organization" (Figure 6C). Among these proteins, we detected a lower abundance of High-Mobility Group A Protein 1 (HMGA1; -1.86 fold change), which is involved in heterochromatin organization and senescence ${ }^{39}$. Also, for High Mobility Group Nucleosome Binding Domain 1 (HMGN1; -1.94 fold change) and 3 (HMGN3; -2.52 fold change), we could detect lower protein levels on the PS-1018 surface. Both HMGN1 and HMGN3 are associated with modifying chromatin structure and influencing transcription 40,41. We also report a reduced expression of the linker histone H1.X (-1.51 fold change), which accumulates in the nucleolus during mitosis and plays a role in chromosome alignment ${ }^{42}$. In the list of proteins with increased expression cultured on the PS-1018 surface, we noted the chaperonin-containing T-complex 2 (CCT2; 4.44 fold change), CCT5 (2.79 fold change), and CCT6A (1.34 fold change), chaperone proteins which can target telomere-associated proteins thereby assisting in telomere maintenance ${ }^{43}$. Related to this, we find increased expression of $\mathrm{X}$ Ray Repair Cross Complementing 6 (XRCC6; 2.25 fold change), which plays a role in DNA repair and telomere maintenance as well ${ }^{44}$. In the list, we also detected a higher presence of Methyl-CpG Binding Domain Protein 2 (MBD2; 3.51 fold change), which represses transcription from methylated promoter regions ${ }^{45}$. Of interest, this protein also recruits HDAC proteins ${ }^{46}$, which might thereby contribute to the lower acetylation levels we observe.

To gain further insight into how micro-topographies influence chromosome organization, we again selected microarray data from surface PS-281 and investigated the presence of genes associated with the GO term "chromosome organization" (Figure 6D). A list of these genes is 

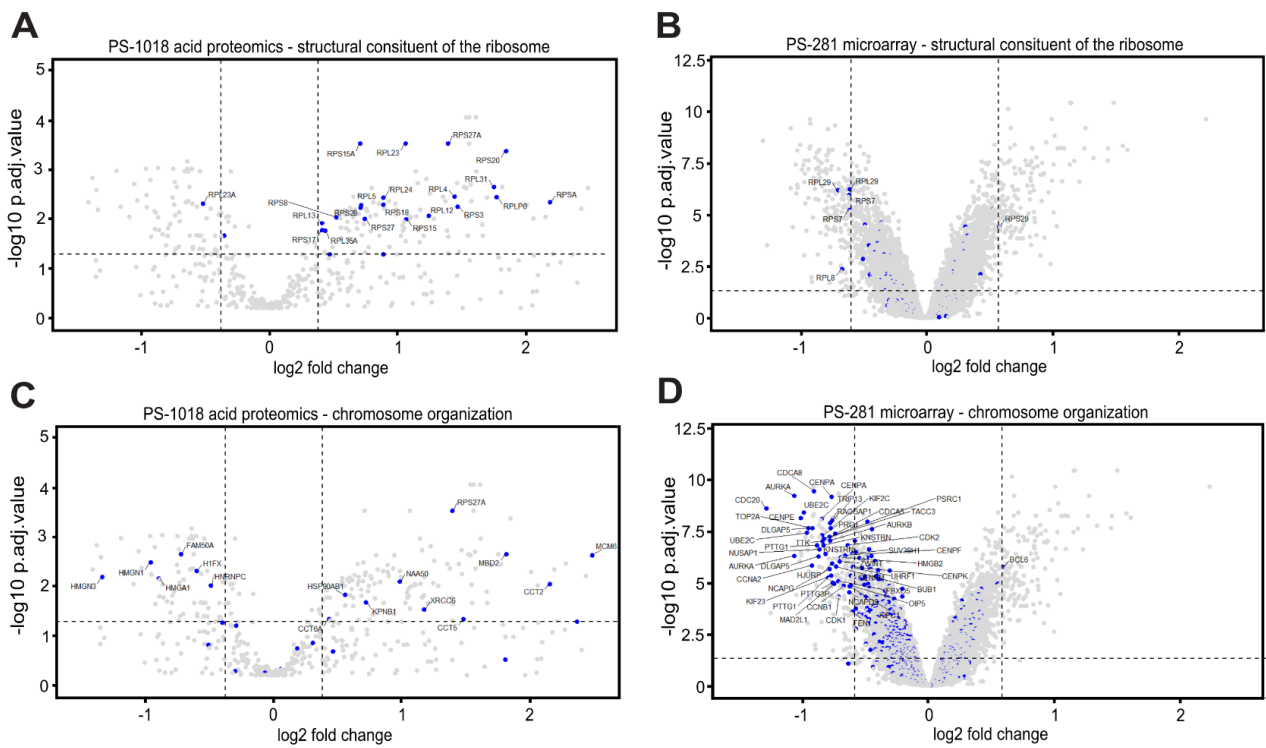

Figure 6: Micro-topographies strongly affect expression levels of genes associated with ribosomal and chromosome organization. A-B) Volcano plot representation of proteomics performed on proteins derived from the acid extraction of MSCs cultured on the PS-1018 surface. Highlighted in blue are proteins associated with the GO term "structural constituent of the ribosome", and "chromosome organization". DEG cut-off was determined at a 1.3 fold change and an adjusted P-value of 0.05 . C-D) Volcano plot representation of microarray performed on isolated RNA derived from MSCs cultured on the PS-281 surface. Highlighted in blue are proteins associated with the GO term "structural constituent of the ribosome", and "chromosome organization". DEG cut-off is determined at a 1.5 fold change and an adjusted P-value of 0.05 .

found in Supplementary Table 3. Here, we found a downregulation of 41 genes and an upregulation of one gene associated with this GO term. In the list of the downregulated genes, we find aurora kinase A (AURKA; -2.09 fold change), and AURKB (-1.71 fold change), both proteins located near the centrosome where they are involved in chromosome movement and organization 47,48 . We also detected a downregulation of multiple centromere proteins (CENP), including CENPA (-1.81 fold change), CENPE (-2.01 fold change), CENPF (-1.57 fold change), CENPK (-1.51 fold change), and CEMPM (-1.53 fold change). These proteins are specialized $\mathrm{H} 3$ variants located at the centrosome, providing unique chromatin regions distinct from traditional euchromatin and heterochromatin ${ }^{49}$. Related to this, we observed a downregulation of Holliday Junction Recognition Protein (HJURP; -1.7 fold change), which is involved in incorporating CENPA in centromeres ${ }^{50}$. We also detected a downregulation of the histonemodifying enzyme suppressor of variegation 3-9 homolog 1 (SUV39H1; -1.52 fold change), which might be involved in the lower levels of $\mathrm{H} 3 \mathrm{~K} 9 \mathrm{Me} 2$ we observed with the histone mass spectrometry data ${ }^{51}$. Related to this, polycomb complex protein regulator of cytokinesis 1 (PRC1; -1.78 fold change) was downregulated, known for its role in the spatial organization of chromatin ${ }^{52}$, and playing a crucial role in cytokinesis ${ }^{53}$. These examples demonstrate that the nuclear deformation that micro-topographies elicit coincides with an altered profile of proteins 
involved in chromatin maintenance and chromosome formation, which is indicative of reduced cell division.

\section{Micro-topographies lower the proliferation potential of MSCs and induce quiescent-like conditions}

A majority of the genes associated with chromosome organization are associated with proliferation. For example, the CENP proteins that play essential roles in centromere formation are also required for proper cell division. This is further emphasized by compiling a differential gene network of the PS-281 microarray data through the STRING database. Of the 248 genes, 85 were associated with the GO term "cell cycle" and clustered together due to their high connectivity with each other based on integration of all publicly available sources of proteinprotein interaction information (Fig. 7A). Amongst this list, we notice a downregulation of cyclin A2 (CCNA2; -1.89 fold change), and cyclin B1 (CCNB1; -1.59 fold change), which are strongly associated with cell division ${ }^{54}$. Reduced proliferation speed of cells on microtopographies was observed before by our group ${ }^{55}$, and is also reported to occur on other biomaterials as well such as on adhesive islands ${ }^{17}$, and reported to be associated with chromatin condensation ${ }^{13}$. Since a considerable amount of these genes are associated with the p53 transcription factor (Supplementary Figure 7), we wondered if micro-topographies initiate a reduced expression of cell cycle genes through p53 activation. For this, we utilized HEK293T cells that exhibit reduced p53 activity through binding with the large- $T$ antigen, and the p53 null cell line SaOS-2, and compared their proliferation speed on micro-topographies with MSCs and U2OS cells. Of interest, we observed significantly reduced proliferation speeds for all cell types (Fig. 7B), indicating that the upstream pathways leading towards a reduction of cell cycle genes are p53 independent, thus warranting further investigation in the pathways involved.

Next, we were interested in determining if the phenotypical changes that micro-topographies exert are permanent or reversible when MSC are cultured back on a flat surface. Determining this is important for understanding the implications of an MSC culture on micro-topographies and how these can be harnessed for tissue engineering applications. For this, we cultured MSCs for three weeks on the PS-1018 surface, after which we reseeded them on a flat surface. Of interest, we found that MSCs cultured from the PS-1018 surface on a flat surface retained a spread-out morphology, indistinguishable from MSCs that were solely cultured on a flat surface (Fig. 7C). Subsequently, we tested if proliferation speed could return towards normal levels. We found through a Cyquant proliferation assay that MSCs after three weeks of culture on the micro-topographies could retain similar proliferation speeds as MSCs cultured on a flat surface (Fig. 7D), indicating that specific phenotypical characteristics that micro-topographies exert on MSCs are reversible.

After determining that micro-topographies induce lower and reversible proliferation, a higher presence of proteins associated with telomere maintenance, and a reduction of nucleoli, which is associated with longevity ${ }^{56}$, we hypothesized that micro-topographies promote maintenance of multipotency in MSCs. For this, we seeded MSCs for three weeks on the PS-1018 surface, 
after which we seeded them on a flat surface and assessed their potential to differentiate towards the osteogenic and adipogenic lineage compared to cells that were cultured for three weeks on a regular flat surface. Of interest, we found that MSCs on the long-term PS-1018 surface had an improved differentiation potential, as shown by an increased presence of mineralization and fat deposits compared to MSCs cultured on flat (Fig. 7E). These findings suggest that microtopographies induce a quiescent-like, multipotent phenotype in MSCs, which coincides with an epigenetic signature associated with lower proliferation speeds and cell division.

A

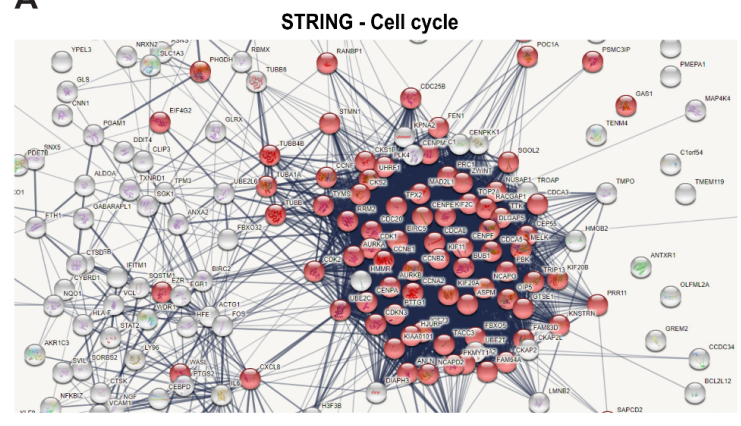

C

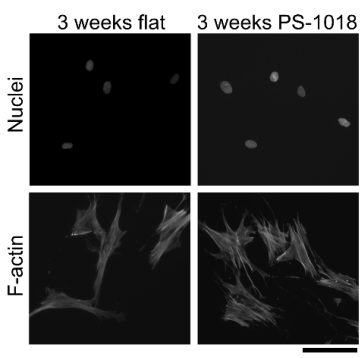

B

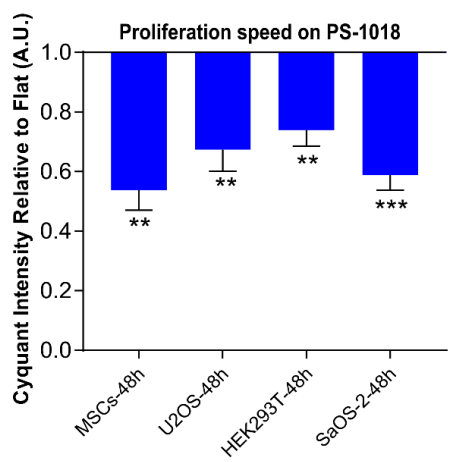

E
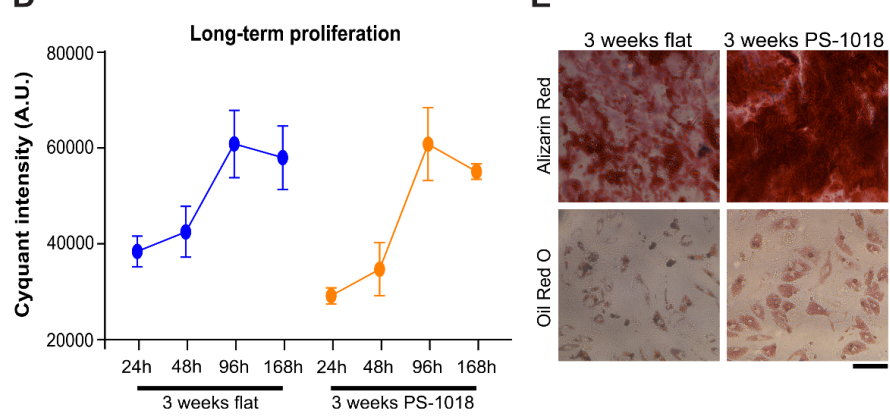

Figure 7: Micro-topographies lower proliferation speed and elicit a reversible phenotype with retained multipotency. A) STRING gene network list compiled from the PS-281 microarray data hints towards a strong association with cell cycle genes. Depicted is a STRING gene network of which red dots are associated with the GO term "cell cycle". B) micro-topographies lower proliferation speed for multiple cell types, as shown for MSCs, U2OS, HEK293T, and the p53-/- cell line Saos-2. C) MSCs cultured for 3 weeks on the micro-topography reversibly adapt their spindle-shaped morphology back towards a spread out morphology characteristic for cells grown on a flat surface. D) MSCs cultured for 3 weeks on micro-topographies retain adapt normal proliferation speeds compared to cells cultured on flat. E) MSCs retain a higher degree of multipotency when cultured on micro-topographies for 3 weeks compared to MSCs cultured on a flat surface. Scale bars represent $100 \mu \mathrm{m}$. 


\section{Discussion}

Our research demonstrates that micro-topographies alter nuclear area, affect histone acetylation levels, and both chromosomal-associated and histone-modifying proteins. Specifically, we found that micro-topographies induce lower histone acetylation levels. This is in contrast to previous reports where fibroblasts cultured on micro-grooves show elevated histone acetylation levels ${ }^{57}$. This demonstrates that biomaterials can profoundly differ in the epigenetic landscape they induce and could be used as a benchmark in the future to predict the response of cells on biomaterials. An interesting follow-up study in this regard is to investigate if the reprogramming efficiency of fibroblasts towards iPSCs is hindered on our micro-topographies due to lower acetylation levels. On adhesive islands, a similar link is confirmed between cell area and acetylation ${ }^{58}$. Furthermore, 3D cultures lead to a reduction in nuclear size, subsequently reducing acetylation levels ${ }^{16}$. These observations are extendable beyond physical cues, e.g., changing the chemistry on the surface leads to a reduction in nuclear area and subsequent chromatin compaction ${ }^{59}$, while altering stiffness levels reduces nuclear area and acetylation ${ }^{60}$. These studies demonstrate that biomaterials have a profound impact on nuclear architecture, and further research is needed to elucidate the biological impact they elicit. In this regard, the observation that micro-topographies can fine-tune acetylation levels is interesting if certain applications require a specific level of global genomic acetylation levels.

A novel finding in this study is the reduced presence of nucleoli when cells are cultured on microtopographies. Nucleoli are associated with rRNA processing and are directly involved in ribosome synthesis. Although we did not directly measure ribosome content, their presence is directly associated with proliferation since cell division requires sufficient production of ribosomal components for protein synthesis. This supports our hypothesis that microtopographies induce a quiescent-like phenotype, which is characterized by a loss of cell cycle progression. How physical cues initiate the pathways behind this phenomenon is subject to future investigation. Applications that can arise from this concept can be the maintenance of cellular identity, as we have demonstrated in this study by the maintenance of MSC multipotency. In this regard, lower acetylation levels are associated with a more naïve state, such as in ESCs ${ }^{61,62}$, which is also known to coincide with reduced proliferation speed ${ }^{63}$. Growing sufficient cell numbers for tissue generation might, therefore, be challenging with micro-topographical cell culture. However, since MSCs can control the behavior of other cells through paracrine signaling, micro-topographies could be interesting for co-culture applications, or the harvest of cell media for clinical applications ${ }^{64}$. In this context, depending on the structural properties of the micro-topographies, growth factor composition can be regulated ${ }^{65}$.

Although we used state-of-the-art histone mass-spectrometry, other techniques can further increase the biological consequences that micro-topographies elicit on the epigenetic landscape. For example, ChIP-Seq can provide information on which parts of the genome are affected by the reduced acetylation and other modifications we measured. Furthermore, it would be interesting to investigate how micro-topographies affect chromatin folding and structure. Since 
we observed higher intensity levels of $\mathrm{H} 3 \mathrm{~K} 9 \mathrm{Me} 2$ on the periphery on some cells, utilizing techniques that identify DNA that comes into contact with the nuclear periphery can give us more insights into the structural organization of the genome ${ }^{28}$. Also, other techniques, such as Hi-C or advanced SEM imaging, can allow us to gain additional insights into this organization 66,67 . In the future, utilizing these techniques in a biomaterial context will be useful for gaining essential insights into how biomaterials affect the epigenetic landscape.

\section{Materials and Methods}

\section{Surface fabrication}

A detailed description of the surface fabrication procedures can be found elsewhere ${ }^{68}$. In short, the inverse pattern of the topographies was etched into a silicon wafer by directional reactive ion etching (DRIE). To facilitate demolding procedures, the wafer was coated with a layer of Trichloro(1H,1H,2H,2H-perfluorooctyl)silane (FOTS, Sigma-Aldrich). Polydimethylsiloxane (PDMS; Down Corning) was cured on the silicon wafer to generate a positive mold and was subsequently used as a template to create a second negative mold in Ormostamp polymer (micro resist technology Gmbh), which serves as the mold for hot embossing the polystyrene (PS) films (Goodfellow). The hot embossing procedure was carried out at $140{ }^{\circ} \mathrm{C}$ for $5 \mathrm{~min}$, and a pressure of $10 \mathrm{Bar}$, with a demolding temperature of $90^{\circ} \mathrm{C}$. Before cell culture, the PS topographies were treated with oxygen plasma to improve cell adhesion for $30 \mathrm{~s}$ at $0.8 \mathrm{mbar}, 50 \mathrm{sccm} \mathrm{O}$, and 100 W. Quality of the fabricated imprints was assessed using a Keyence VK-H1XM-131 profilometer.

\section{Cell culture}

Adipose-derived human mesenchymal stem cells (AD-hMSCs) used in this study were purchased from Lonza. AD-hMSCs were isolated from a 42-year-old female. All methods were performed in accordance with the relevant ethical guidelines and regulations of the University of Maastricht. Basic medium for AD-hMSCs consists of MEM Alpha GlutaMAX, no nucleosides (Gibco). Basic media was supplemented with 10\% v/v fetal bovine serum (FBS; Sigma), $0.2 \mathrm{mM}$ ascorbicacid-2-phosphate (ASAP), and $10 \mathrm{U} / \mathrm{mL}$ Penicillin/Streptomycin. Cells were grown in a humid atmosphere at $37^{\circ} \mathrm{C}$ and $5 \% \mathrm{CO}_{2}$.

\section{Microarray study and pathways analysis}

Bone marrow-derived human MSCs were seeded on topography PS-281 for 24 hours in basic medium at a density of 15,000 cells $/ \mathrm{cm}^{2}$ in 24 well plates in three replicas. Total RNA was isolated using the Nucleospin RNA isolation kit (Macherey-Nagel). Then, from $100 \mathrm{ng}$ of RNA, cRNA was synthesized using the Illumina TotalPrep RNA amplification kit. Both RNA and cRNA quality was verified on a Bioanalyzer 2100 (Agilent). Microarrays were performed using Illumina HT-12 v4 expression Beadchips. $750 \mathrm{ng}$ of cRNA was hybridized on the array overnight, after which the array was washed and blocked. Then, through the addition of streptavidin Cy-3, a fluorescent signal was developed. Arrays were scanned on an Illumina 
Beadarray reader, after which raw intensity values were background corrected in BeadStudio (Illumina). Further data processing and statistical testing were performed using the online portal arrayanalysis.org. The probe-level raw intensity values were quantile normalized and transformed using variance stabilization (VSN). A detection threshold of 0.01 was used for reducing the number of false positives. A linear modeling approach with empirical Bayesian methods, as implemented in Limma package, was applied for differential expression analysis of the resulting probe-level expression values. P-values were corrected for multiple testing using the Benjamini and Hochberg method. Genes with a corrected p-value below 0.05 were considered differentially expressed.

To construct a gene network of the DEGs, we applied an online STRING analysis (https://string-db.org/). Only DEGs from the microarray data with a fold change higher than 1.5 and an adjusted p-value lower than 0.05 were included in the list.

\section{Histone isolation and propionylation}

Adipose-derived mesenchymal stem cells were seeded on flat and PS-1018 surfaces and cultured for $4,24,48$, and 72 hours in basic medium at a density of 15,000 cells $/ \mathrm{cm}^{2}$ in 6 -well plate format. Cells from a plate were pooled together, after which the cell pellet was flash frozen. This process was repeated 5 times to obtain 6 replicas. Histone extraction was performed using a direct acid protocol as described elsewhere ${ }^{69}$. Therefore, 2 million cells were resuspended in 250 $\mu \mathrm{l}$ of $0.4 \mathrm{~N} \mathrm{HCl}$ and incubated for 4 hours on a rotator at $4{ }^{\circ} \mathrm{C}$. The histones were precipitated with 33\% trichloroacetic acid (TCA) while incubated on ice for 30 minutes. Afterwards, the precipitated histones were washed twice with ice cold acetone to remove residual TCA. A small amount of the extracted histone fraction, corresponding to 200.000 cells was quantified by gelelectrophoresis using a 8-16\% TGX gel (Biorad) and a commercially available bovine histone standard (Roche). The remaining purified histones of each sample were vacuum dried and propionylated ${ }^{70,71}$. In short, histones were dissolved in $20 \mu \mathrm{L} 1 \mathrm{M}$ triethylammonium bicarbonate (TEAB) buffer (Sigma-Aldrich), pH 8.5 before $20 \mu \mathrm{L}$ of propionylation reagent $(1: 79(\mathrm{v} / \mathrm{v})$ of a propionic anhydride and 2-propanol mixture) was added, for an incubation of 30 minutes at room temperature. This was followed by adding $20 \mu \mathrm{l}$ milliQ water (Merck Millipore) for 30 minutes at $37^{\circ} \mathrm{C}$. Histones were then digested overnight at $37^{\circ} \mathrm{C}$ using trypsin (Promega) at an enzyme/histone ratio of 1:20 (m/m) in $500 \mathrm{mM}$ TEAB, supplemented with $\mathrm{CaCl}_{2}$ and ACN to a final concentration of $1.0 \mathrm{mM}$ and $5 \%$ respectively. Subsequently, the propionylation reaction was carried out again to cap peptide $\mathrm{N}$-termini. Overpropionylation at serine, threonine and tyrosine was reversed by resuspending the vacuum dried sample in $50 \mu \mathrm{L} 0.5 \mathrm{M} \mathrm{NH}_{2} \mathrm{OH}$ and 15 $\mu \mathrm{L} \mathrm{NH} \mathrm{NH}_{4} \mathrm{OH}$ at $\mathrm{pH} 12$ for 20 minutes at room temperature after which $30 \mu$ pure formic acid was added. Finally, the samples were vacuum dried again and stored for LC-MS analysis.

\section{Liquid chromatography and mass spectrometry analysis}

The samples were resuspended in $0.1 \%$ Formic Acid (FA) in water to achieve a histone concentration of $200 \mathrm{ng} / \mu \mathrm{L}$. Internal standards Beta-Galactosidase (B-gal) (Sciex) and MPDS2 
(Waters) were spiked in a concentration of $12.5 \mathrm{fmol} / \mu \mathrm{L}$. Quality control (QC) samples were made by mixing $1 \mu \mathrm{l}$ of each biological sample. Histones were analysed by micro-RPLC-MS using a nanoACQUITY UPLC system (Waters) coupled to a Synapt G2-Si Q-TOF (Waters) mass spectrometer. An ACQUITY Symmetry C18 $180 \mu \mathrm{m}$ x $20 \mu \mathrm{m}$ trap column (Waters) was combined with an ACQUITY M-Class CSH ${ }^{\text {TM }}$ C18 300 $\mu \mathrm{m}$ x $100 \mathrm{~mm}$ column (Waters) at a flow rate of $5 \mu \mathrm{L} / \mathrm{min}(0.1 \%$ FA spiked with $3 \%$ DMSO). A 90 minute gradient from $3-40 \%$ ACN in $0.1 \% \mathrm{FA}$ was applied with a total scan time of 105 minutes per sample. The sample list was randomized and interspersed with QC injections. Each cycle consisted in one full MS1 scan (m/z 50-5000) of 200 millseconds (ms), followed by an MS2 scan of $100 \mathrm{~ms}$. The 10 most intense precursor ions were selected from a single MS survey scan for MS2 fragmentation.

\section{Mass spectrometry data analysis}

Data analysis was done as described earlier ${ }^{72}$. The raw data was imported and aligned in Progenesis QI for Proteomics (QIP) 4.1 (Nonlinear Dynamics, Waters) before feature detection was performed. For identification, the five tandem MS2 spectra closest to the elution apex were selected for each feature and exported as .mascot generic format file (.mgf) to search with Mascot 2.6.1 (Matrix Science). The obtained .mgf is searched using three types of searches: (a) a standard search for the identification of non-propionylated histones and to validate the presence of the internal standards B-gal and MPDS2; (b) an error tolerant search to identify all the proteins in the sample; (c) multiple sequential searches with six different PTM sets for the identification of modified histone peptides. The different PTM sets were selected using an established concept, to reduce the ambiguity in annotation. The following parameters were set in all three searches: two missed cleavages, peptide mass tolerance of $10 \mathrm{ppm}$ and a fragment mass tolerance of 50 ppm. For search a) trypsin is set as digestion enzyme, while $\operatorname{ArgC}$ (only cleaves after arginine residues) is set for the error tolerant search (b) and the sequential searches (c). Next, the identification files were exported as .xml files and imported in Progenesis QIP. Manual validation of the annotated histone features was performed to resolve isobaric near-coelution. Afterwards, the same procedure for identification was repeated and finally these identification results were also imported in Progenesis QIP.

\section{Immunocytochemistry}

After cell culture, cells were washed with phosphate-buffered saline (PBS; Merck) and fixed with $4 \%\left(\mathrm{w} / \mathrm{v}\right.$ ) paraformaldehyde (Sigma-Aldrich) for $5 \mathrm{~min}$ at $37^{\circ} \mathrm{C}$. After a washing step, cells were permeabilized with $0.01 \%$ (v/v) Triton X-100 (Acros Organics) and blocked with goat serum (1:100; Sigma-Aldrich) in PBT (PBS + 0.02\% Triton-X-100, 0.5\% BSA) for 1 h. Afterward, cells were incubated with the primary antibody in PBT for $1 \mathrm{~h}$. After a washing step, cells were incubated with a secondary antibody conjugated to an Alexa Fluor (1:500; ThermoFisher) in PBT. In conjunction, Phalloidin conjugated to an Alexa Fluor (1:500; ThermoFisher) in PBT was added to the sample for $1 \mathrm{~h}$. After washing, the nucleus was counterstained with Hoechst33258 (1:1000; Sigma-Aldrich) for $10 \mathrm{~min}$. After a subsequent washing step, surfaces were mounted on glass cover slides with mounting media (Dako). All washing steps were 
performed in triplicate with PBT. Primary antibodies used in this study are: anti-acetyl-histone H3 antibody (1:200; Millipore; 06-599), anti-acetyl histone H4 antibody (1:200; Abcam; ab177790), anti-H3K9Me2 (1:200; Abcam; ab1220), anti-nucleophosmin antibody (1:200; Abcam; ab37659), anti-fibrillarin (1:100; Abcam; ab4566), and anti-nucleolin antibody (1:200; ThermoFisher; ZN004).

\section{Image analysis}

Fixed samples were inverted, and fluorescent images were acquired through the glass coverslip using a fully automated Nikon Eclipse Ti-U microscope in combination with an Andor Zyla 5.5 4MP camera. Fluorescent images were analyzed through CellProfiler 3.1.8 ${ }^{24}$, applying custommade pipelines. After illumination corrections, the morphology of the nucleus was captured by the Otsu adaptive thresholding method applied on the Hoechst 33258 image channel. Subsequently, cell morphology was determined by applying propagation and Otsu adaptive thresholding on the Phalloidin image channel. Cells touching the border of the image were filtered out of the dataset. Missegmentation artifacts were removed by applying an arbitrary threshold on nuclei and cell area. After background correction, the intensity values of the target of interest were calculated inside the nuclear area. The imaging software Fiji was used for image visualization ${ }^{73}$.

\section{MSC osteogenic and adipogenic differentiation}

To assess if the AD-MSCs were multipotent during the experiments, we investigated their potential for differentiation towards the osteogenic and adipogenic lineage. Differentiation of AD-hMSCs towards the osteogenic lineage was achieved by seeding AD-hMSCs at a density of $5 \times 10^{3}$ cells $/ \mathrm{cm} 2$. After $24 \mathrm{~h}$, the medium was changed with either a control or mineralization medium. The mineralization media is basic media supplemented with $10 \% \mathrm{v} / \mathrm{v}$ fetal bovine serum (FBS), $10 \mathrm{U} / \mathrm{mL}$ Penicillin/Streptomycin, with $10 \mathrm{nM}$ dexamethasone (Sigma) and 10 $\mathrm{mM} \beta$-glycerol phosphate (Sigma), while control medium includes the same components except for dexamethasone. The media was refreshed every 2-3 days, and after 21 days, cells were fixed overnight at $4{ }^{\circ} \mathrm{C}$ with $4 \%$ formaldehyde (VWR) in PBS. Afterward, osteogenesis was assessed through staining mineralized deposits with a $2 \%$ Alizarin Red solution $(\mathrm{pH}=4.2)$ for 2 min. Excess staining was washed off with demineralized water (Supplementary Fig. 9A).

Differentiation of AD-hMSCs towards the adipogenic lineage was achieved by seeding ADhMSCs at a density of $15 \times 10^{3}$ cells $/ \mathrm{cm} 2$. After $24 \mathrm{~h}$, the medium was replaced with either a control or adipogenic medium. The adipogenic media consist of basic media supplemented with $10 \% \mathrm{v} / \mathrm{v}$ fetal bovine serum (FBS), $10 \mathrm{U} / \mathrm{mL}$ Penicillin/Streptomycin, $0.5 \mathrm{mM} 3$-isobutyl-1methylxanthine (Sigma), $0.2 \mathrm{mM}$ indomethacin (Sigma), $10 \mu \mathrm{g} / \mathrm{mL}$ Insulin (Sigma), and $1 \mu \mathrm{M}$ dexamethasone (Sigma). The control medium consisted solely of basic media with $10 \% \mathrm{v} / \mathrm{v}$ fetal bovine serum (FBS) and $10 \mathrm{U} / \mathrm{mL}$ Penicillin/Streptomycin. The media was refreshed every 2-3 days, and after 21 days, cells were fixed overnight at $4{ }^{\circ} \mathrm{C}$ with $3.7 \%$ formaldehyde (VWR), 0.01 $\mathrm{g} / \mathrm{ml} \mathrm{CaCl}_{2} \cdot 2 \mathrm{H}_{2} \mathrm{O}$ (Merck) in PBS. Afterward, adipogenesis was assessed by rinsing the fixation 
solution with demineralized water, and subsequently incubating the substrates in a $60 \%(\mathrm{v} / \mathrm{v}) 2$ propanol (VWR) for $5 \mathrm{~min}$. Fat droplets were stained through a freshly filtered solution of $0.3 \%$ (w/v) Oil Red O dissolved in 60\% (v/v) 2-propanol (VWR). Afterward, the substrates were washed in triplicate with demineralized water (Supplementary Fig. 9B).

\section{Statistical analysis}

Statistical analysis was performed with GraphPad Prism (GraphPad Prism Software Incl. San Diego, USA). For image analysis, a two-sided T-test was applied to determine significant intensity differences elicited between flat and topography. Each data point represents a measurement from an individual cell. Image experiments were replicated with similar results. For histone mass-spectrometry data, one-way ANOVA was applied to compare relative histone abundancies.

\section{Acknowledgments}

SV, LBB, and BV acknowledge the financial support of the Dutch province of Limburg. SV is supported by the European Union's Horizon 2020 Programme (H2020-MSCA-ITN-2015; Grant agreement 676338).

\section{Data Availability Statement}

The data that support the findings of this study are available from the corresponding author upon reasonable request.

\section{Competing Interests}

The authors declare no competing interests.

\section{References}

1. Holtzer, H., Abbot, J., Lash, J. \& S., H. The Loss of Phenotypic Traits by Differentiated Cells in vitro, I. Dedifferentiation of Cartilage Cells. PNAS 46, 1533-1542 (1960).

2. Vermeulen, S. et al. Identification of topographical architectures supporting the phenotype of rat tenocytes. Acta Biomater. 83, 277-290 (2019).

3. Yang, Y. K., Ogando, C. R., See, C. W., Chang, T. \& Barabino, G. A. Changes in phenotype and differentiation potential of human mesenchymal stem cells aging in vitro. Stem Cell Res. Ther. 9, 131 (2018).

4. Eom, Y. W. et al. The role of growth factors in maintenance of stemness in bone marrow-derived mesenchymal stem cells. Biochem. Biophys. Res. Commun. 445, 16-22 (2014).

5. Flaim, C. J., Chien, S. \& Bhatia, S. N. An extracellular matrix microarray for probing cellular differentiation. Nat. Methods 2, 119-125 (2005).

6. Gilbert, P. et al. Substrate elasticity regulates skeletal muscle stem cell self-renewal in culture. Science (80-. ). 329, 1078-1081 (2011).

7. Engler, A. J., Sen, S., Sweeney, H. L. \& Discher, D. E. Matrix Elasticity Directs Stem Cell Lineage Specification. Cell 126, 677-689 (2006).

8. Shao, Y. et al. Self-organized amniogenesis by human pluripotent stem cells in a biomimetic implantation-like niche. Nat. Mater. (2016). doi:10.1038/NMAT4829 
9. McBeath, R., Pirone, D. M., Nelson, C. M., Bhadriraju, K. \& Chen, C. S. Cell shape, cytoskeletal tension, and RhoA regulate stem cell lineage commitment. Dev. Cell 6, 483-495 (2004).

10. Kilian, K. A., Bugarija, B., Lahn, B. T. \& Mrksich, M. Geometric cues for directing the differentiation of mesenchymal stem cells. Proc. Natl. Acad. Sci. 107, 4872-4877 (2010).

11. Abagnale, G. et al. Surface topography enhances differentiation of mesenchymal stem cells towards osteogenic and adipogenic lineages. Biomaterials 61, 316-326 (2015).

12. Hulshof, F. F. B. et al. Mining for osteogenic surface topographies: In silico design to in vivo osseo-integration. Biomaterials 137, 49-60 (2017).

13. Versaevel, M., Grevesse, T. \& Gabriele, S. Spatial coordination between cell and nuclear shape within micropatterned endothelial cells. Nat. Commun. 3, 1-11 (2012).

14. Guo, M. et al. Cell volume change through water efflux impacts cell stiffness and stem cell fate. PNAS c, 1-10 (2017).

15. Ikeda, H., Sone, M., Yamanaka, S. \& Yamamoto, T. Structural and spatial chromatin features at developmental gene loci in human pluripotent stem cells. Nat. Commun. 8, 1-13 (2017).

16. Beye, J. Le, Xu, R., Sun-Young, L. \& Celeste M. Nelson1, Aylin Rizki1, Jordi Alcaraz1, and M. J. B. Cell shape regulates global histone acetylation in human mammary epithelial cells. Exp Cell Res 454, 42-54 (2007).

17. Singhvi, R. et al. Engineering Cell Shape and Function. Science (80-. ). 264, 696-698 (1994).

18. Werner, M. et al. Surface Curvature Differentially Regulates Stem Cell Migration and Differentiation via Altered Attachment Morphology and Nuclear Deformation. Adv. Sci. 4, 1-11 (2017).

19. Jain, N. \& Vogel, V. Spatial confinement downsizes the inflammatory response of macrophages. Nat. Mater. (2018). doi:10.1038/s41563-018-0190-6

20. Unadkat, H. V et al. An algorithm-based topographical biomaterials library to instruct cell fate. Proc. Natl. Acad. Sci. U. S. A. 108, 16565-70 (2011).

21. Moe, A. A. K. et al. Microarray with micro- and nano-topographies enables identification of the optimal topography for directing the differentiation of primary murine neural progenitor cells. Small 8 , 3050-3061 (2012).

22. Hu, J. et al. High-Throughput Mechanobiology Screening Platform Using Micro- and Nanotopography. Nano Lett. 55, 9557-9561 (2017).

23. Hulsman, M. et al. Analysis of high-throughput screening reveals the effect of surface topographies on cellular morphology. Acta Biomater. 15, 29-38 (2015).

24. McQuin, C. et al. CellProfiler 3.0: Next-generation image processing for biology. PLoS Biol. 16, 1-17 (2018).

25. Barski, A. et al. Resource High-Resolution Profiling of Histone Methylations in the Human Genome. Cell 129, 823-837 (2007).

26. Ferrari, K. J. et al. Polycomb-Dependent H3K27me1 and H3K27me2 Regulate Active Transcription and Enhancer Fidelity. Mol. Cell 53, 49-62 (2014).

27. Ruan, $\mathrm{K}$. et al. Histone $\mathrm{H} 4$ acetylation required for chromatin decompaction during DNA replication. Sci. Rep. 5, 1-10 (2015).

28. Kind, J. et al. Single-cell dynamics of genome-nuclear lamina interactions. Cell 153, 178-192 (2013).

29. Vigushin, D. M. et al. Trichostatin A is a histone deacetylase inhibitor with potent antitumor activity against breast cancer in vivo. Clin. Cancer Res. 7, 971-976 (2001).

30. Göttlicher, M. et al. Valproic acid defines a novel class of HDAC inhibitors inducing differentiation of transformed cells. EMBO J. 20, 6969-6978 (2001).

31. Görisch, S. M., Wachsmuth, M., Tóth, K. F., Lichter, P. \& Rippe, K. Histone acetylation increases chromatin accessibility. J. Cell Sci. 118, 5825-5834 (2005).

32. Savkur, R. S. \& Olson, M. O. J. Preferential cleavage in pre-ribosomal RNA by protein B23 endoribonuclease. Nucleic Acids Res. 26, 4508-4515 (1998).

33. Murano, K., Okuwaki, M., Hisaoka, M. \& Nagata, K. Transcription Regulation of the rRNA Gene by a Multifunctional Nucleolar Protein, B23/Nucleophosmin, through Its Histone Chaperone Activity. Mol. Cell. Biol. 28, 3114-3126 (2008).

34. Okuwaki, M., Matsumoto, K., Tsujimoto, M. \& Nagata, K. Function of nucleophosmin/B23, a nucleolar acidic protein, as a histone chaperone. FEBS Lett. 506, 272-276 (2001). 
35. Toiiervey, D., Lehtonen, H., Jansen, F., Kern, H. \& Hurt, E. C. Temperature sensitive mutations demonstrate roles for yeast fibrillarin in pre-rRNA processing and ribosome assembly. Cell 72, 443-457 (1993).

36. Ginisty, H., Amalric, F. \& Bouvet, P. Nucleolin functions in the first step of ribosomal RNA processing. EMBO J. 17, 1476-1486 (1998).

37. Ma, N. et al. Nucleolin functions in nucleolus formation and chromosome congression. J. Cell Sci. 120, 2091-2105 (2007).

38. Zhou, X., Liao, W. J., Liao, J. M., Liao, P. \& Lu, H. Ribosomal proteins: Functions beyond the ribosome. J. Mol. Cell Biol. 7, 92-104 (2015).

39. Chicas, A. et al. A Novel Role for High-Mobility Group A Proteins in Cellular Senescence and Heterochromatin Formation. Cell 126, 503-514 (2006).

40. Murphy, K. J. et al. HMGN1 and 2 remodel core and linker histone tail domains within chromatin. Nucleic Acids Res. 45, 9917-9930 (2017).

41. West, K. L. et al. HMGN3a and HMGN3b, Two Protein Isoforms with a Tissue-specific Expression Pattern, Expand the Cellular Repertoire of Nucleosome-binding Proteins. J. Biol. Chem. 276, 25959-25969 (2001).

42. Takata, H. et al. H1.X with different properties from other linker histones is required for mitotic progression. FEBS Lett. 581, 3783-3788 (2007).

43. Freund, A. et al. Proteostatic control of telomerase function through TRiC-mediated folding of TCAB1. Cell 159, 1389-1403 (2014).

44. Hsu, H. L., Gilley, D., Blackburn, E. H. \& Chen, D. J. Ku is associated with the telomere in mammals. Proc. Natl. Acad. Sci. U. S. A. 96, 12454-12458 (1999).

45. Hendrich, B. \& Bird, A. Identification and Characterization of a Family of Mammalian MethylCpG Binding Proteins. Mol. Cell. Biol. 18, 6538-6547 (1998).

46. Müller-Tidow, C. et al. Loss of expression of HDAC-recruiting methyl-CpG-binding domain proteins in human cancer. Br. J. Cancer 85, 1168-1174 (2001).

47. Liu, D., Vader, G., Vromans, M. J. M., Lampson, M. A. \& Lens, S. M. A. Sensing Chromosome Bi-Orientation Kinase from Kinetochore Substrates. Science 323, 1350-1353 (2009).

48. Giet, R. et al. Drosophila Aurora A kinase is required to localize D-TACC to centrosomes and to regulate astral microtubules. J. Cell Biol. 156, 437-451 (2002).

49. Sullivan, B. A. \& Karpen, G. H. Centromeric chromatin exhibits a histone modification pattern that is distinct from both euchromatin and heterochromatin. Nat Struct Mol Biol. 11, 1076-1083 (2004).

50. Foltz, D. R. et al. Centromere-Specific Assembly of CENP-A Nucleosomes Is Mediated by HJURP. Cell 137, 472-484 (2009).

51. Wang, T. et al. Crystal Structure of the Human SUV39H1 Chromodomain and Its Recognition of Histone H3K9me2/3. PLoS One 7, (2012).

52. Schoenfelder, S. et al. Polycomb repressive complex PRC1 spatially constrains the mouse embryonic stem cell genome. Nat. Genet. 47, 1179-86 (2015).

53. Jiang, W. et al. PRC1: A human mitotic spindle-associated CDK substrate protein required for cytokinesis. Mol. Cell 2, 877-885 (1998).

54. Gong, D. \& Ferrell, J. E. The Roles of Cyclin A2 , B1 , and B2 in Early and Late Mitotic Events. Mol. Biol. Cell 21, 3149-3161 (2010).

55. Beijer, N. R. M. et al. Dynamic adaptation of mesenchymal stem cell physiology upon exposure to surface micropatterns. Sci. Rep. 9, 1-14 (2019).

56. Tiku, V. \& Antebi, A. Nucleolar Function in Lifespan Regulation. Trends Cell Biol. 28, 662-672 (2018).

57. Downing, T. L. et al. Biophysical regulation of epigenetic state and cell reprogramming. Nat. Mater. 12, 1154-1162 (2013).

58. Jain, N., Iyer, K. V., Kumar, A. \& Shivashankar, G. V. Cell geometric constraints induce modular gene-expression patterns via redistribution of HDAC3 regulated by actomyosin contractility. Proc. Natl. Acad. Sci. 110, 11349-11354 (2013).

59. Vergani, L., Grattarola, M. \& Nicolini, C. Modifications of chromatin structure and gene expression following induced alterations of cellular shape. Int. J. Biochem. Cell Biol. 36, 1447-1461 (2004).

60. Killaars, A. R. et al. Extended Exposure to Stiff Microenvironments Leads to Persistent Chromatin Remodeling in Human Mesenchymal Stem Cells. Adv. Sci. 1801483, (2019). 
61. De Clerck, L. et al. Untargeted histone profiling during naive conversion uncovers conserved modification markers between mouse and human. Sci. Rep. 9, 1-11 (2019).

62. Mierlo, G. Van et al. Integrative Proteomic Profiling Reveals PRC2- Dependent Epigenetic Crosstalk Maintains Ground- State Pluripotency. Cell Stem Cell 24, 1-15 (2019).

63. Jasencakova, Z., Walter, J., Schubert, I., Meister, A. \& Turner, B. M. Histone H4 Acetylation of Euchromatin and Heterochromatin Is Cell Cycle Dependent and Correlated with Replication Rather Than with Transcription. Plant Cell 12, 2087 (2007).

64. Doorn, J., Moll, G., Le Blanc, K., van Blitterswijk, C. \& de Boer, J. Therapeutic Applications of Mesenchymal Stromal Cells: Paracrine Effects and Potential Improvements. Tissue Eng. Part B Rev. 18, 101-115 (2012).

65. Leuning, D. G. et al. The cytokine secretion profile of mesenchymal stromal cells is determined by surface structure of the microenvironment. Sci. Rep. 8, 1-9 (2018).

66. Stevens, T. J. et al. 3D structures of individual mammalian genomes studied by single-cell Hi-C. Nature 544, 59-64 (2017).

67. Chen, B. et al. Three-dimensional positioning and structure of chromosomes in a human prophase nucleus. Sci. Adv. 3, 1-9 (2017).

68. Zhao, Y. et al. High-definition micropatterning method for hard, stiff and brittle polymers. Mater. Sci. Eng. C 71, 558-564 (2017).

69. Govaert, E. et al. Extracting histones for the specific purpose of label-free MS. Proteomics 16, 2937-2944 (2016).

70. Meert, P., Govaert, E., Scheerlinck, E., Dhaenens, M. \& Deforce, D. Pitfalls in histone propionylation during bottom-up mass spectrometry analysis. Proteomics 15, 2966-2971 (2015).

71. Meert, P. et al. Tackling aspecific side reactions during histone propionylation: The promise of reversing overpropionylation. Proteomics 16, 1970-1974 (2016).

72. Willems, S. et al. Flagging False Positives Following Untargeted LC-MS Characterization of Histone Post-Translational Modification Combinations. J. Proteome Res. 16, 655-664 (2017).

73. Schindelin, J. et al. Fiji - an Open platform for biological image analysis. Nat. Methods 9, 241 (2009). 


\section{Supplementary Figures}

A

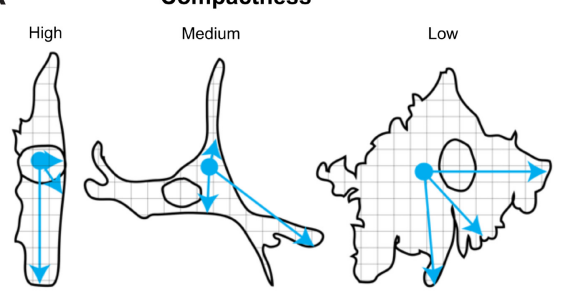

B



C

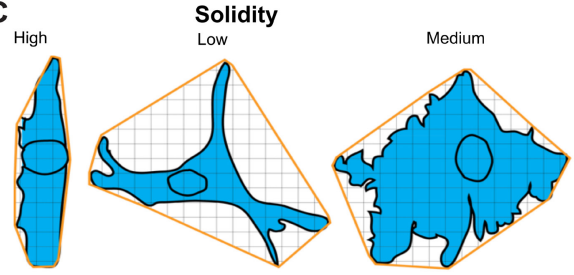

$\square$-Area of the cell $\square$-Area of the convex hul

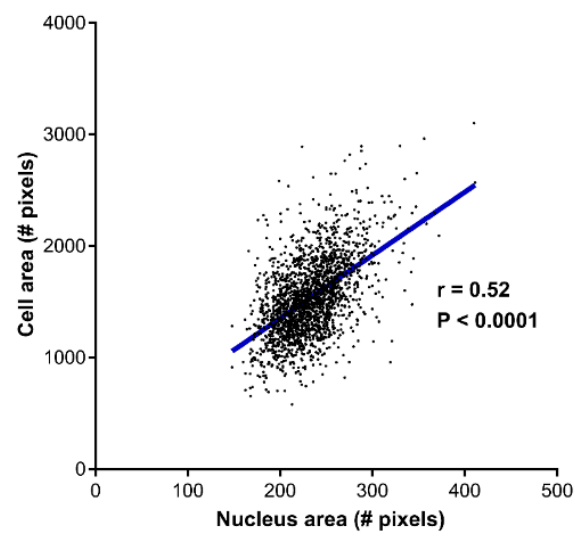

Supplementary Figure 1: Schematic illustrations of the morphological parameters compactness, eccentricity, and solidity. A-B) Elongated cells exhibit high compactness and eccentricity values. C) Branched cells typically exhibit low solidity values.

Supplementary Figure 2: Micro-topographies that induce lower cell area also induce lower nuclear area. Each dot represents the median cell and nuclear size elicited by an individual microtopography. Pearson $r=0.52, \mathrm{P}<0.0001$. 
A

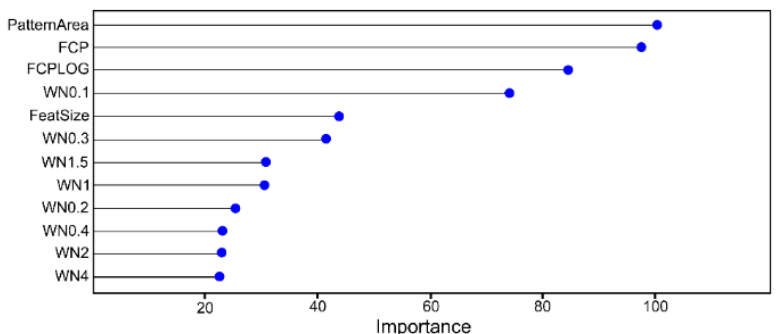

B

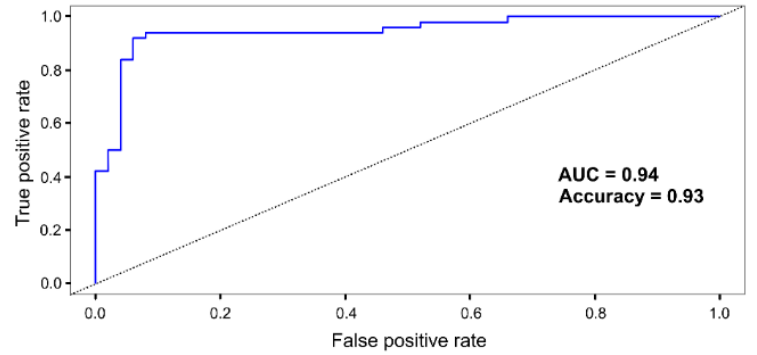

Supplementary Figure 3: Random forest algorithms parameters. A) Random forest algorithms associate pattern area, FCP, FCPLOG, and WN0.1 as the most important feature parameters for determining the nuclear area. B) These parameters could be predicted with a high area under the curve (AUC) of 0.94 , and an accuracy of 0.93 .

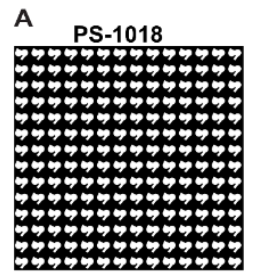

B

Nuclei


Supplementary Figure 4: Surface PS-1018 induces elongated morphological characteristics in MSCs. A) In silico design of the PS-1018 surface. B) MSCs cultured on the PS-1018 surface exhibit elongated characteristics and a mild reduction in cell and nuclear size. F-actin stained with Phalloidin and nuclei counterstained with 



Supplementary Figure 5: Immunostaining of $\mathrm{H} 3 \mathrm{~K} 9 \mathrm{Me} 2$ reveals a heterogeneous intensity pattern when MSCs are cultured on the PS1018 surface. H3K9Me2 levels of MSCs exhibiting elevated intensity levels are more located at the nuclear periphery. Scale bar represents $50 \mu \mathrm{m}$.
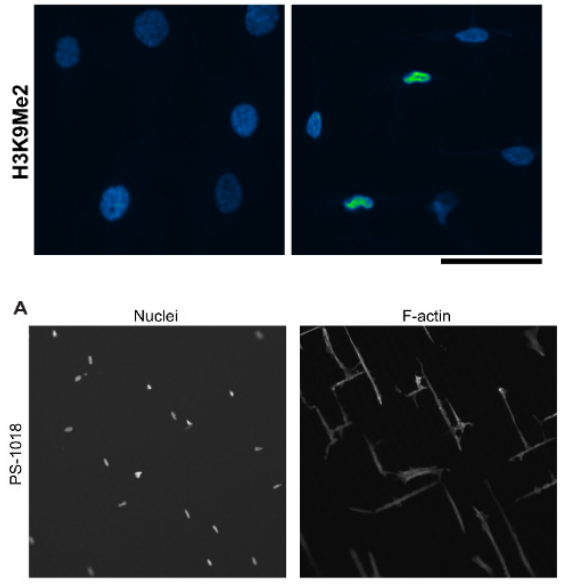

Supplementary Figure 6: Comparison of morphological characteristics of MSCs cultured on the PS-1018 and PS-281 surface. A) Morphological characteristics of MSCs culture on the PS-1018 platform. B) Morphological characteristics of MSCs culture on the PS-281 platform. F-actin stained with Phalloidin and nuclei counterstained with Hoechst33258. Scale bar represents $200 \mu \mathrm{m}$.

B
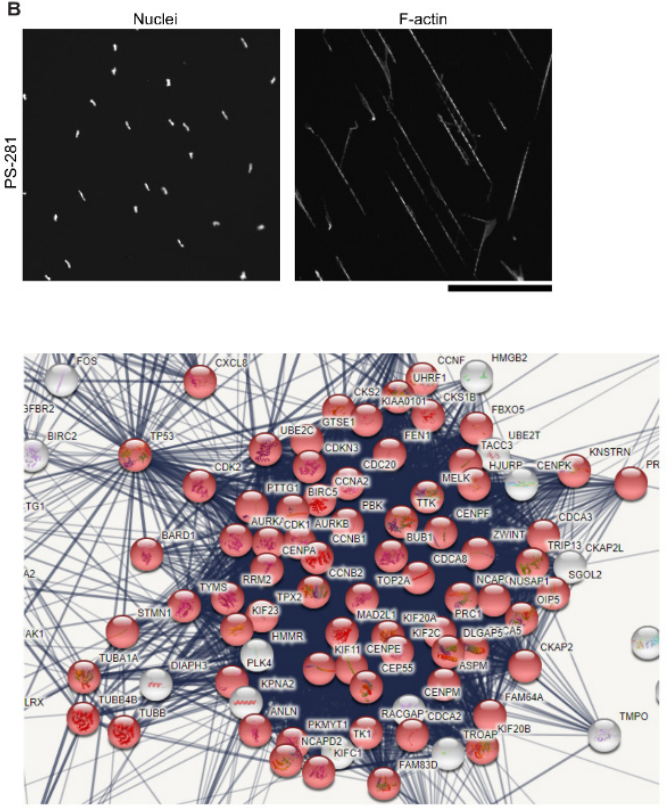

Supplementary Figure 7: STRING gene network of the microarray data set of surface PS281. Highlighted in red are DEGs associated with the GO term "cell cycle". TB53 (p53) is included in the network to demonstrate the interconnectedness of $\mathrm{p} 53$ with the DEGs. 


\section{Chapter VIII}

\section{Extending Biomaterial Surface}

\section{Topographical Design Space through} Natural Surface Reproduction

Steven Vermeulen, ${ }^{1,2}$, Floris Honig ${ }^{1}$, Aliaksei Vasilevich ${ }^{2}$, Nadia Roumans ${ }^{1}$, Manuel Romero ${ }^{3}$, Paul Williams ${ }^{3}$, Aurélie Carlier ${ }^{1}$, Jorge Alfredo Uquillas ${ }^{2}$, Jan de Boer $^{2}$

${ }^{1}$ Laboratory for Cell Biology-Inspired Tissue Engineering, MERLN Institute, University of Maastricht, Maastricht, The Netherlands

${ }^{2}$ BioInterface Science Group, Department of Biomedical Engineering and Institute for Complex Molecular Systems, University of Eindhoven, Eindhoven, The Netherlands.

${ }^{3}$ School of Life Sciences, Centre for Biomolecular Sciences, University of Nottingham, NG7 2RD Nottingham, The United Kingdom

Submitted for publication 


\section{Abstract}

Surface topography guides cell behavior and is a tool to endow biomaterials with bioactive properties. The large number of possible designs makes it challenging to find the optimal surface structure to induce a specific cell response. The TopoChip platform is currently the largest collection of topographies with 2176 in silico designed micro-topographies. Still, it is exploring only a small part of design space due to boundary conditions of the design algorithm and the surface engineering strategy. Inspired by diversity of natural surfaces, we assessed whether to which extend we could expand topographical design space and cellular responses using natural surfaces. To this end, we replicated twenty-six plant and insect surfaces in polystyrene and quantified their surface properties using white light interferometry and image analysis. Next, we quantified mesenchymal stem cell morphology and the pattern of Pseudomonas aeruginosa attachment and compared it to previous data from TopoChip screens. Our results show that natural surfaces extended the TopoChip design space and induced MSC morphologies and bacterial attachment patterns not previously observed on the TopoChip. In the future, we will train our design algorithms with results obtained by natural surface imprint experiments to further explore design space and bio-active properties of surface topography. 


\section{Introduction}

Biological surfaces are interfaces between an organism and its environment and is the location where the organism deals with the chemical and physical reality of the outside world. Through evolution, bio-interfaces have acquired functional characteristics to support survival, which can be of chemical nature, as seen in plant waxes that decrease moisture loss ${ }^{1}$ and protect against UV radiation. ${ }^{2}$ On the other hand, topographical characteristics give surfaces very interesting and useful properties. For example, the setae on gecko feet allow movement on smooth vertical walls ${ }^{3}$, and setae mimetics are used as dry and reversible adhesive for both robotic ${ }^{4}$ and biomedical applications. ${ }^{5}$ Superhydrophobicity, a material property observed on certain plant surfaces such as the Holy Lotus and Red Rose, is caused by hierarchical micro- and nanostructures, and results in self-cleaning surfaces. ${ }^{6,7}$ Mosquitos use specialized superhydrophobic nanostructures on their eyes to prevent the nucleation of fog droplets ${ }^{8}$, and the tooth-like scales on shark skin provide drag reduction, anti-biofouling, and superoleophobicity which protects against oil spills. ${ }^{9,10}$ Antimicrobial nano-structures on cicada wings may reduce the infection risk of implants ${ }^{11,12}$ and through inspiration from the Nepenthes pitcher plant, lubrication fluids cover micro/nanostructured medical devices with repellent and self-cleaning surfaces. ${ }^{13-16}$ These fascinating evolutionary and bioengineered structures demonstrate the richness in natural surface topographical bioactivity and begs research to use topographical design to improve the performance of materials for industrial and clinical applications.

Surface topography is currently used as a design tool to improve biomaterials in prosthetic dentistry, tissue-engineering and regenerative medicine but also to improve biocompatibility of medical devices. ${ }^{17-19}$ Surface topography has a very large design space, which we define as the universe of surface architectures, and ranges from randomly introduced roughness ${ }^{20}$ to designed groove patterns. ${ }^{21}$ Additionally, topographies exist as pillars ${ }^{22}$ or complex geometries ${ }^{23,24}$, while curvature provides convex shapes. ${ }^{25}$ All these structures, found both in micro- and nanometer dimensions, are known to affect the cells that are in contact with them.

The large topographical design space complicates the quest for the optimal topography for a specific application. To this end, high-throughput topography screening (HTS) platforms were developed and many novel bioactive surfaces have been discovered. ${ }^{26-28}$ However, HTS of platforms still have their limitations because their design strategy only covers a small part of the whole design space. For example, structures in both nano- and micrometer dimensions are rarely present in the same platform and variable roughness levels are not included in a high-throughput setting. Man-made designed surface structures are frequently presented in an organized pattern, yet disorder also profoundly influences cell behavior. ${ }^{29,30}$ The bottleneck in producing a more diverse spectrum of surface topographies is not in the in silico design possibilities, where algorithms such as neural networks could aid the design and fine tuning of surface topographies ${ }^{24}$, but rather the technical limitations in surface topography manufacturing. Even though state of the art techniques such as two-photon stereo-lithography can handle the fabrication of 
complex shapes ${ }^{31}$, its limited writing speed remains unsuitable for high-throughput applications. Photolithography, on the other hand, is mostly a 2D technique unsuitable to design complex structures. We hypothesized that we can increase design space by using natural surfaces as a mold, and solvent casting as a microfabrication technique to replicate them into materials of interest, in our case tissue culture polystyrene. Many reports describe the replication of natural surfaces ${ }^{32-36}$, and in this work we used a quantitative approach to compare design space coverage with artificially generated surface structures.

We investigated the combination of multiple natural surfaces in one platform for the creation of a novel architectural design sub-space not commonly found in artificial platforms. We sampled a diverse set of 26 plant and animal surfaces, reproduced their structures in polystyrene, and investigated their potential for controlling cell behavior. We demonstrated that the structural diversity from the natural surfaces surpasses that of the micro-topographical TopoChip platform and show stem cell and bacterial bioactivity in this array of natural surface topographies not seen before.

\section{Results and discussion}

\section{Natural surface architectures exhibit a wide design variety}

We selected sixteen plant and ten insect surfaces with a diverse set of surface properties based on reported phenomena such as super-hydrophobicity, anti-fouling or light reflection (see Figure S1 and Figure S2). Observation of the unprocessed specimens using scanning electron microscopy revealed an interesting variety in surface topography. For example, the Calla lily petal surface exhibited interconnected cuticular folds with ridges of $1 \mu \mathrm{m}$ in height (Fig. 1a, top row, first left panel), which is very different from the petal surface of the Red Rose, which has parallel-aligned hierarchical structures of $20 \mu \mathrm{m}$ high micropapillae and nanofolds (Figure 1a, top row, center panel). Holy Lotus, known for its superhydrophobicity, has heptagonal inclinations, a nano rough surface and convex micro curvature resulting in a $10 \mu \mathrm{m}$ high pillar in the center (Figure 1a, top row, first panel right). On the rice surface, we find pillar structures with longitudinal ridges (Figure 1a, bottom row, first panel left). The Huechys incarnata wing is an interesting case, where merged pillars were present, which gave rise to a variable structure size between $500 \mathrm{~nm}$ and $5 \mu \mathrm{m}$ in diameter (Figure 1a, bottom row, center panel). Variable pillar sizes could be found on cicadas such as the Yanga adriana. (Figure 1a, bottom row, first panel right). We classified plant surfaces in five groups: a) cuticular folds with low elevation, b) cuticular folds with high elevation, c) oriented structures, d) complex structures and e) microroughness (Figure S3). Three classes were observed on animal surfaces: a) nanopillars, b) pits, and c) curved surfaces (Figure S4).

\section{Natural surface architectures can be imprinted with high fidelity into polystyrene}

We chose polystyrene, the standard material, used in the culture dish, as reference chemistry in which to compare cell-material interaction. In order to transfer the natural surface structures into polystyrene (PS), we used a relatively easy and fast technique that only requires the use of 
glass slides, binder clips, and a conventional oven (Figure 1b). ${ }^{37}$ PDMS was cast upon the natural surface, after which it was cured at room temperature for $24 \mathrm{~h}$. The PDMS containing the

a



b



C

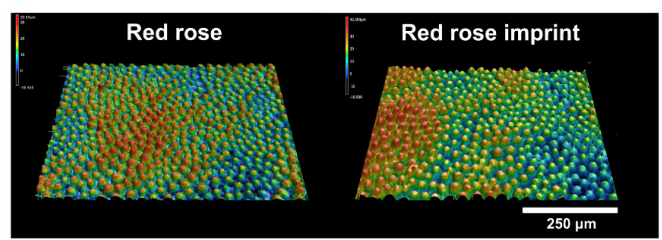

d

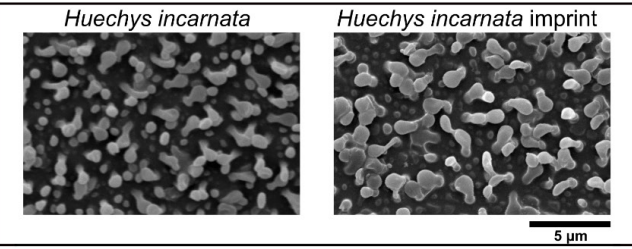

Figure 1. Natural surfaces exhibit a large topographical variety. a) Examples of SEM images of leaves and insect wings utilized in this study. b) Fabrication scheme to imprint natural surface designs onto polystyrene. c) Leave imprints can be transferred to polystyrene with high fidelity as seen in the profilometric images of Red Rose and its imprint counterpart. d) Insect wing imprints can be transferred to polystyrene with high fidelity as seen in the profilometric images of Huechys incarnata and its imprint counterpart. negative imprint of the natural topographies was peeled off the natural surface (Figure 1b, top row and Figure S5A) and a 'sandwich' was created containing glass slides, the PDMS imprint and a polystyrene sheet (Figure 1b, bottom row and Figure S5B). The construct was placed in the oven and after half an hour, the construct was cooled to room temperature and the PS imprint peeled off the PDMS.

We next compared the profilometric images of the imprints and original specimens and found that the imprints were successfully transferred with high fidelity from natural surfaces into PS. Examples are provided for the leaves of the Red Rose (Figure 1c), and Huechys incarnata (Figure 1d). Sub-micron structures present on the cuticular folds of the Red Rose and Holy Lotus were also successfully replicated (Figures S6 and S7).

After observing the correct imprinting of natural surface topographies onto PS, we assessed the superhydrophobic properties of both the Red Rose and Holy Lotus imprints by measuring the water contact angle. No significant differences were seen between the natural and PS imprints (Figure S8). The rose petal effect, resulting in pinning of a water droplet, was demonstrated by inverting and tilting the imprint. The Lotus effect, which is characteristic of the rapid rolling of water droplets on the surface was also seen on the PS Holy Lotus imprint (data not shown). These examples demonstrate that also the biophysical properties of natural topographies can be replicated on PS. 


\section{Natural surface architectures occupy a different part of the design space and induce distinct cell morphology compared to TopoChip micro-topographies}

The objective of this study was to determine whether natural surface topographies occupy a different part of design space than our library of randomly generated TopoChip topographies. ${ }^{23}$ We plotted 199 randomly sampled TopoChip surfaces and the twenty-six natural surfaces using principal component analysis (PCA), based on shape features extracted using CellProfiler software. ${ }^{38} \mathrm{PCA}$ is a dimension reduction technique that allows visual representation of variation in data, with distance between dots representing dissimilarity. Interestingly, TopoChip topographies formed four distinct clusters: a cluster that represents micro-topographies with low pattern areas, clusters with intermediate pattern areas, and two clusters with a high pattern area yet distinguishable by micro-topographies that exhibit either small or large feature sizes (Figure 2a). In Figure 2b, we represent examples of in silico design of such surfaces and in Figure 2c, a profilometric example of the PS-1018 surface is presented, which exhibits an intermediate pattern area. On the other hand, natural surfaces formed a third 'linear' cluster with micro- and nanotopographies with low and high heights, with distinct pattern densities, demonstrating that natural surfaces represent an unexplored part of design space (Figure 2d).

Based on published reports, cell shape and surface topography are highly correlated ${ }^{39-41}$, and we wondered if a larger design space also leads to yet unobserved cell shape features. To this end, we seeded hMSCs both on PS imprints of the natural surfaces and onto 28 TopoChip topographies which were selected to span the whole range of cell shape variation on the TopoChip. ${ }^{42}$ F-actin and DNA were stained, and quantitative cell and nucleus area, and cell compactness and solidity data were extracted for all surfaces (Figure 3). ${ }^{38}$ In Figure 3a, the effect of topography on MSC cell and nucleus area is compared relative to MSCs on flat PS. In general, hMSCs seeded on natural surfaces show substantially smaller cell and nuclear sizes



d

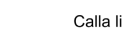

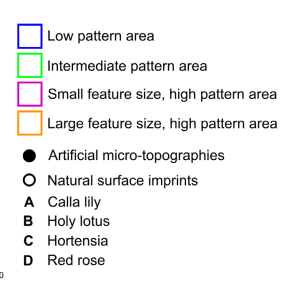

Holy lotus

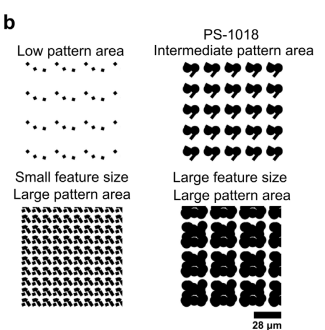

$\overline{28 \mu \mathrm{m}}$



Red rose

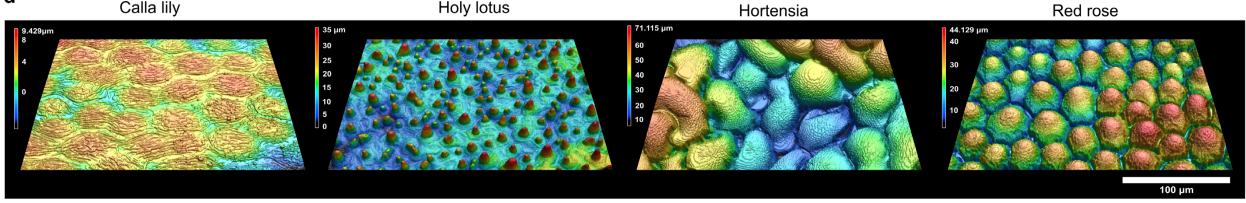

Figure 2. Natural surfaces expand the topographical design space. a) Principal component analysis of the TopoChip and natural surfaces images reveals distinct clustering representing different material design. b) In silico design of artificial micro-topograpies of different clusters. c) Profilometric image of surface PS-1018, exhibiting an intermediate pattern area. d) Examples of Profilometric images of natural surface topographies. 
compared to cells cultured on flat with the strongest effects seen for cells cultured on microtopographies. Cell compactness relates to cell elongation, and cell solidity is inversely correlated to cell branching and radial filopodia protrusion. A high compactness value indicates a more elongated cell, and a high solidity value indicates that the cell is less branched and less filopodial protrusions extend radially. Here, we see a strong distinction between cells cultured on natural and artificial topographies. Natural surfaces reduced cell branching and radial filopodia protrusion with most cells on natural surfaces with solidity values of more than one (Figure 3b). Furthermore, cells were more elongated on artificial micro-topographies. Representative fluorescence images of cells grown on flat, onion, and TopoChip surface 11 facilitated the visualization of size differences (Figure 3c), while the Shining Leaf Shafer Beetle and surface 3 assisted in visualizing differences in solidity and compactness (Figure 3d). In general, the analysis of cell compactness and solidity between designed and natural surfaces shows that natural topographies induced cell morphologies distinct from those observed on the TopoChip.

\section{Natural surface topographies induce distinct profiles of Pseudomonas aeruginosa attachment}

Next, we investigated differences in bacteria attachment patterns between designed and natural surface imprints to gain novel understandings in bacteria-material interaction. Pseudomonas aeruginosa was chosen as a model bacteria since it is frequently associated with infections after surgical procedures and urinary-tract infections in hospitals. ${ }^{43-46}$ Psendomonas aeruginosa were cultured on the natural surfaces and on PS TopoChips for $4 \mathrm{~h}^{47}$ and assessed for bacterial attachment using fluorescent microscopy. Quantification of bacterial attachment will be discussed in future studies; here we focus on differences in distribution of bacterial attachment using the texture parameters in CellProfiler software ${ }^{38}$. The spatial relation features (SRF) 1 and 2 derived from the natural surfaces and 26 randomly sampled TopoChip are plotted in Figure 3e, which clearly demonstrated that the natural surfaces occupy a different area of distribution than the TopoChip surfaces. In general, we noted more uniform and ordered bacterial distribution on TopoChip surfaces (Figure 3f). 



e



f

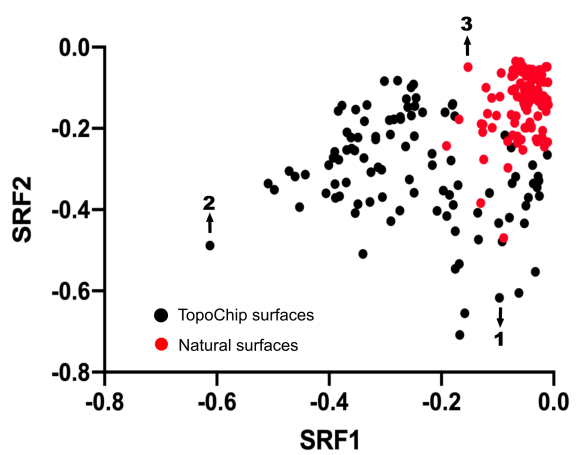

Bacterial attachment

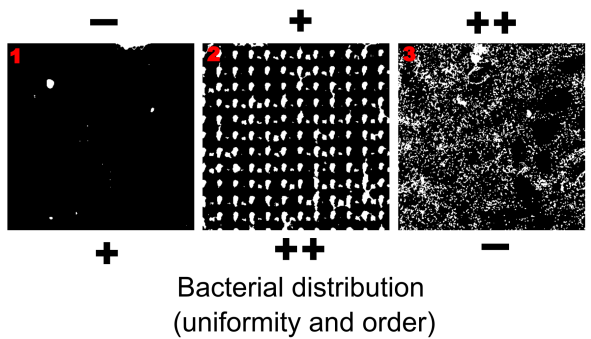

Figure 3. TopoChip and natural surfaces elicit distinct effects on the shape of MSCs and the attachment distribution of Pseudomonas aeruginosa. a) Cell and nucleus area quantification of hMSCs on TopoChip and natural surfaces. b) Cell compactness and solidity quantification of hMSCs on TopoChip and natural surfaces. c) Visual representation of the change in cell and nucleus area of hMSCs on TopoChip and natural surfaces. d) Visual representation of the change in cell compactness and solidity of hMSCs on TopoChip and natural surfaces. e) Spatial relationship feature plot of Psendomonas aeruginosa on TopoChip and natural surfaces. $\mathbf{f}$ ) Visual representation of the change in bacterial attachment and distribution of Pseudomonas aeruginosa on TopoChip and natural surfaces. 


\section{Conclusion}

In this work, we transferred features of natural surfaces to cell culture platforms to increase the topographical design space. We demonstrated that natural surfaces (i) can be transferred with high fidelity in polystyrene, (ii) occupy an unexplored area of topographical design space relative to the TopoChip, (iii) uniquely alter the size and shape of adipose-derived human mesenchymal stem cells, and (iv) affect the spatial distribution of Pseudomonas aeruginosa. The TopoChip design only encompasses a local minimum in the total material design space. With natural surfaces we can step out of our local minimum and bring new and unique features into the design space. In this manuscript, we used only twenty-six natural surfaces, a minuscule subset of all the plants and insects on the planet. In future work, we will further expand the topographical design space at the Tiputini Biodiversity Station in the Ecuadorian Amazon rainforest, one of the most biodiverse tropical forests in the world ${ }^{48-51}$, and scan natural surfaces with a portable profilometer based on interferometry to obtain digital representation of natural surfaces..$^{52}$ Afterwards, we would like to explore deep learning algorithms developed in house 53,54 and elsewhere 55 that decouple complex topographical information of natural surfaces, and provide guides in selecting surfaces that cover uncharted topographical territory. Two-photon lithography can then be used to generate the surfaces and analyze their bio-active properties.

\section{Materials and methods}

\section{Fabrication of artificial micro-topographies in the TopoChip.}

A detailed description describing the fabrication of the micro-topographical surfaces can be found elsewhere ${ }^{56}$. In brief, the inverse pattern was etched on a silicon wafer by directional reactive ion etching (DRIE). To facilitate subsequent demoulding procedures, the silicon waver was coated with a layer of perfluorooctyltrichlorosilane (FOTS, Sigma-Aldrich). Polydimethylsiloxane (PDMS; Sylgard ${ }^{\circledR} 184$ Elastomer Kit, Dow Corning) in a 10:1 silicon/curing agent ratio (w/w) was cured on the waver at $85{ }^{\circ} \mathrm{C}$ for 6 hours to generate a positive mold. OrmoStamp hybrid polymer (micro-resist technology Gmbh) was subsequently poured on the PDMS imprint together with a borofloat waver containing a layer of Ormoprime (micro-resist technology Gmbh). UV treatment allowed the cross-linking of a final negative Ormostamp imprint, which is used as a template for the hot embossing step at $140{ }^{\circ} \mathrm{C}$ for 10 minutes to generate a polystyrene imprint.

\section{Fabrication of natural surface topographies in the TopoChip}

Dried insects used in this study were purchased from the supplier "The Bugmaniac" (http://www.thebugmaniac.com). The Holy Lotus (Nelumbo nucifera), Rice (Oryza sativa), Plaintain Lily (Hosta sp.), and Hardy Canna (Thalia dealbata) were kindly donated from the botanical garden "Hortus Botanicus" of Leiden (https://www.hortusleiden.nl). Other plants were purchased at the local flower-shop. Replication of natural surfaces was achieved through a two-step fabrication process. ${ }^{37}$ The first step included the fabrication of a polydimethylsiloxane 
(PDMS) mold, which contains the negative imprint of the natural surface. A sample of the fresh natural surface of choice was fixed onto a petri dish using double-sided adhesive tape (Double Fix, Bison). The silicon resin and curing agent (Sylgard ${ }^{\circledR} 184$ Elastomer Kit, Dow Corning) were mixed in a 10:1 ratio (w/w) and cast onto the natural surface. Subsequently, the liquid mixture was degassed and cured for 48 hours at room temperature to prevent heat damage. Finally, the solid PDMS mold was separated from the natural surface. In the second step, the PDMS mold served as a template for transferring the structures into PS using hot embossing. To achieve this, an assembly was made in which a $190 \mu \mathrm{m}$ thick PS film was placed on the PDMS mold clamped between two Teflon sheets and microscope slides to apply constant pressure. To cross the glasstransition of the PS film, the entire system was inserted in an oven for $1 \mathrm{~h}$ at $150{ }^{\circ} \mathrm{C}$. Afterward, the assembly was removed from the oven and cooled down to room temperature to allow the PS film to solidify. Finally, the PS surface with transferred surface topography was carefully peeled from the PDMS mold.

\section{Scanning electron microscopy imaging}

Scanning electron microscopy was used for examination of the surface topography of natural surfaces and quality control of their respective PS replicas. Preparation of fresh natural samples consisted of fixation ( $2 \%$ glutaraldehyde in $0.1 \mathrm{M}$ cacodylate buffer) for $1 \mathrm{hr}$. Subsequently, the samples were washed three times in cacodylate $(0.1 \mathrm{M})$ before dehydration by immersion in a graduated series of ethanol in water $(50 \%, 70 \%, 90 \%, 100 \%)$. Then, samples were critical point dried (EM CPD300, Leica) using 15 exchange cycles with slow gas out and heating speed settings. All samples were mounted on SEM stubs using carbon conductive adhesive tape. Finally, samples were sputter-coated with gold (Sputter Coater 108auto, Cressington) for 100 seconds prior to imaging using a scanning electron microscope (XL-30, Philips) at 10kV.

\section{Cell culture}

Adipose-derived human mesenchymal stem cells (AD-hMSCs) were purchased from Lonza, which were isolated from a 42-year-old female. Basic medium for AD-hMSCs consisted of MEM Alpha GlutaMAX (Gibco), fetal bovine serum (FBS, 10\% v/v), ascorbic-acid-2-phosphate (ASAP, $0.2 \mathrm{mM}$ ), and penicillin/streptomycin $\left(10 \mathrm{U} \mathrm{mL}^{-1}\right)$. Cells were grown at $37^{\circ} \mathrm{C}$ in a humid atmosphere at $5 \% \mathrm{CO}_{2}$. For experimental purposes, cells were seeded on flat and topographical surfaces at a density of 10000 cells $/ \mathrm{cm}^{2}$ unless stated otherwise.

\section{Fluorescent imaging}

After cell culture, cells were washed with phosphate-buffered saline (PBS, Sigma-Aldrich) before fixation in formaldehyde $(3.6 \% \mathrm{v} / \mathrm{v})$ for $10 \mathrm{~min}$ at $37^{\circ} \mathrm{C}$. After, cells were washed three times with PBS and permeabilized by the addition of Triton-X-100 $(0.1 \% \mathrm{v} / \mathrm{v})$ in PBS for $10 \mathrm{~min}$. Following this, samples were blocked using serum (1:100) in PBT $(0.02 \%$ Triton-X-100, PBS \& $0.6 \%$ bovine serum albumin) for $1 \mathrm{hr}$. After another washing step, samples were incubated for 1 $\mathrm{h}$ in Phalloidin with a fluorochrome attached to visualize F-actin (1:500, ThermoFisher). Finally, 
Hoechst 33258 (1:1000, ThermoFisher) was used for visualization of nuclei. After three subsequent washing steps, the samples were mounted in Mowiol.

\section{Human mesenchymal stem cell morphology analysis}

Fixed and stained samples were inverted, and fluorescent images were acquired through the glass coverslip using a fully automated Nikon Eclipse Ti-U microscope in combination with an Andor Zyla5.5 four megapixels camera. Fluorescent images were analyzed through CellProfiler 3.1.8 ${ }^{38}$, applying custom-made pipelines. All images were cropped in order to remove out-of-focus objects. Objects touching the border of the subsequent images were filtered out of the dataset. After illumination corrections, the morphology of the nucleus was captured by the Otsu adaptive thresholding method applied on the Hoechst 33258 image channel. Subsequently, cell morphology was determined by applying propagation and Otsu adaptive thresholding on the Phalloidin image channel. Missegmentation artifacts were removed by applying an arbitrary threshold on nuclei and cell size. For visualization purposes, we enhanced the brightness and contrast of the images representing cellular morphologies. The imaging software Fiji was used for image visualization. ${ }^{57}$

\section{Bacteria imaging and data acquisition}

The opportunistic human pathogen Pseudomonas aeruginosa PAO1 was chosen to test performance of natural topography replicas against bacterial surface attachment. PAO1 was routinely grown at $37^{\circ} \mathrm{C}$ in lysogeny broth (LB) or LB agar. Tryptic soy broth (TSB) supplemented with human serum $(10 \% \mathrm{v} / \mathrm{v})$ was used as the growth medium for bacterial topographies attachment assays. Prior to incubation with bacteria, topographies were washed by dipping in distilled water and sterilized in ethanol $(70 \% \mathrm{v} / \mathrm{v})$. The air-dried samples were placed in petri dishes $(60 \mathrm{~mm} \times 13$ $\mathrm{mm}$ ) and incubated statically at $37^{\circ} \mathrm{C}$ in $10 \mathrm{ml}$ of growth medium inoculated with diluted (optical density: OD600 nm = 0.01) bacteria from overnight cultures. After 4 h incubation, topography samples were removed from PAO1 cultures and washed in PBS ( $\mathrm{pH}$ 7.4) to remove loosely attached cells. After rinsing with distilled water, attached cells were stained with SYTO9 $(50 \mu \mathrm{M}$; Molecular Probes, Life Technologies) for $30 \mathrm{~min}$ at room temperature. After staining, topographies were rinsed with distilled water, air-dried and mounted on a glass slide using Prolong antifade reagent (Life Technologies). Topographies were then imaged by confocal laser scanning using a Zeiss LSM 700 microscope (Carl Zeiss, Germany) and $488 \mathrm{~nm}$ laser as light source. Since the bacterial cells may attach at different heights on the micro-patterns, images were initially acquired as $\sim 75 \mu \mathrm{m}$ range Z-stacks ( $1.5 \mu \mathrm{m}$ steps - 50 slides) from the TUs using a 10x objective (Zeiss, EC Plan-Neofluar 10x/0.30 Ph 1). To categorize topographies influencing bacterial attachment, the fluorescence signal from each topography image $(n=12)$ was used for quantification. The mean fluorescence intensity of maximum intensity projections for each Zstack was measured using Fiji-Image 2.0.0 software (National Institutes of Health, US) and all values were normalized to the average fluorescence intensity of the flat surface control. 


\section{PCA analysis}

Greyscale images of the natural surfaces were captured through profilometric imaging through a Keyence VK-H1XM-131 profilometer. Height profiles were represented as grayscale images, where black represented the bottom of the surfaces and white top. For artificial surfaces, pixel values 0 correspondent to the bottom and 10 to the top of the pillars, which matches their height in $\mu \mathrm{m}$. Since the natural surfaces height profile gradually varied from the bottom to the top of the surface, the pixel values also were counted and were arranged in a range from 0 (bottom) to the height value as measured by profilometry. As the input for the algorithm, we have used pixel values directly, 250000 values per surface. Resulting images contained information about the height of the elements as well as their structure on the area of $200 \times 200 \mu \mathrm{m}$ with resolution 500x500 pixels. Profilometry software was further used to correct for sample tiltness. We further corrected images by applying local contrast enhancement algorithm as implemented in sci-kit python 3.7.3 package. Intensity values in the image were normalized further by subtracting minimum pixel value and dividing by max pixel intensity value per surface. The final image was obtained by multiplying a resulting matrix by height value as measured with the profilometer.

\section{Texture quantification}

Spatial distribution of bacteria attachment on the TopoChip and natural surfaces was quantified by image texture features, which were extracted from the thresholded images. Image analysis was performed in CellProfiler 3.0.0.

\section{Texture Feature Selection}

To distinguish texture features that were the most discriminative between TopoChip and natural surfaces, we employed a binary classification algorithm. Particularly we trained the Extreme Gradient Boosting (EGB) model on a hold-out subset that contained over 500 images of the bacteria attachment on either TopoChip or natural surfaces. The accuracy of the trained model was validated using the training set and the accuracy value was 0.98 . An accuracy of 1.0 corresponds to a perfectly performing classification algorithm. We further quantified the importance of the features with the Mean Decrease Accuracy (MDA) algorithm that measures how model accuracy decreases when a feature is excluded from the prediction.

\section{Spatial relationship features}

We identified that the texture feature 'InfoMeas1 for scales 64 and 2' were the most important for distinguishing bacteria attachment between TopoChip and natural surfaces. 'InfoMeas1' is a measure of the total amount of information contained within a region of pixels derived from the recurring spatial relationship between specific intensity values. ${ }^{58}$. We rename them in the text and Figure 3e as Spatial Relationship Feature 1 (SRF1) and Spatial Relationship Feature 2 (SRF2). For visualization purposes, we transformed SRF1 by taking its cubic root. The scatter plot in Figure 3 f represents 100 randomly selected surfaces from both TopoChip and natural groups. 


\section{Acknowledgements}

SV, NH, NR, and JdB acknowledge the financial support of the Dutch province of Limburg. SV is grateful for the support of the European Union's Horizon 2020 Programme (H2020-MSCAITN-2015; Grant agreement 676338). We thank the botanical garden "Hortus Botanicus" of Leiden, the Netherlands, for the donation of plant species, and Louay Waked for his help in material fabrication.

\section{References}

1. Radler, F. Reduction of Loss of Moisture by the Cuticle Wax Components of Grapes. Nature 207, 835-837 (1965).

2. $\quad$ Long, L. M., Prinal Patel, H., Cory, W. C. \& Stapleton, A. E. The maize epicuticular wax layer provides UV protection. Funct. Plant Biol. 30, 75-81 (2003).

3. Gao, H., Wang, X., Yao, H., Gorb, S. \& Arzt, E. Mechanics of hierarchical adhesion structures of geckos. Mech. Mater. 37, 275-285 (2005).

4. Yu, J. et al. Gecko-inspired dry adhesive for robotic applications. Adv. Funct. Mater. 21, 3010 3018 (2011).

5. Palacio, M. L. B., Bhushan, B. \& Schricker, S. R. Gecko-inspired fibril nanostructures for reversible adhesion in biomedical applications. Mater. Lett. 92, 409-412 (2013).

6. Barthlott, W. \& Neinhuis, C. Purity of the sacred lotus, or escape from contamination in biological surfaces. Planta 202, 1-8 (1997).

7. Feng, L. et al. Petal effect: A superhydrophobic state with high adhesive force. Langmuir 24, 4114-4119 (2008).

8. Gao, X. et al. The dry-style antifogging properties of mosquito compound eyes and artificial analogues prepared by soft lithography. Adv. Mater. 19, 2213-2217 (2007).

9. Pu, X., Li, G. \& Huang, H. Preparation, anti-biofouling and drag-reduction properties of a biomimetic shark skin surface. Biol. Open 1-8 (2016). doi:10.1242/bio.016899

10. Bhushan, B. Biomimetics inspired surfaces for drag reduction and oleophobicity/philicity. Beilstein J. Nanotechnol. 2, 66-84 (2011).

11. Watson, G. S. et al. A gecko skin micro/nano structure - A low adhesion, superhydrophobic, anti-wetting, self-cleaning, biocompatible, antibacterial surface. Acta Biomater. 21, 109-122 (2015).

12. Pogodin, S. et al. Biophysical model of bacterial cell interactions with nanopatterned cicada wing surfaces. Biophys. J. 104, 835-840 (2013).

13. Wong, T. S. et al. Bioinspired self-repairing slippery surfaces with pressure-stable omniphobicity. Nature (2011). doi:10.1038/nature10447

14. Epstein, A. K., Wong, T. S., Belisle, R. A., Boggs, E. M. \& Aizenberg, J. Liquid-infused structured surfaces with exceptional anti-biofouling performance. Proc. Natl. Acad. Sci. U. S. A. (2012). doi:10.1073/pnas.1201973109

15. Wilson, P. W. et al. Inhibition of ice nucleation by slippery liquid-infused porous surfaces (SLIPS). Phys. Chem. Chem. Phys. (2013). doi:10.1039/c2cp43586a

16. Leslie, D. C. et al. A bioinspired omniphobic surface coating on medical devices prevents thrombosis and biofouling. Nat. Biotechnol. (2014). doi:10.1038/nbt.3020

17. Cooper, L. F. A role for surface topography in creating and maintaining bone at titanium endosseous implants. J. Prosthet. Dent. (2000). doi:10.1067/mpr.2000.111966

18. Wennerberg, A. \& Albrektsson, T. Effects of titanium surface topography on bone integration: A systematic review. Clin. Oral Implants Res. 20, 172-184 (2009).

19. Tuomi, J. T. et al. In vitro cytotoxicity and surface topography evaluation of additive manufacturing titanium implant materials. J. Mater. Sci. Mater. Med. (2017). doi:10.1007/s10856-017-58631

20. Jaggy, M. et al. Hierarchical Micro-Nano Surface Topography Promotes Long-Term Maintenance of Undifferentiated Mouse Embryonic Stem Cells. Nano Lett. 15, 7146-7154 (2015). 
21. Abagnale, G. et al. Surface topography enhances differentiation of mesenchymal stem cells towards osteogenic and adipogenic lineages. Biomaterials 61, 316-326 (2015).

22. Kolind, K. et al. A combinatorial screening of human fibroblast responses on micro-structured surfaces. Biomaterials 31, 9182-9191 (2010).

23. Unadkat, H. V et al. An algorithm-based topographical biomaterials library to instruct cell fate. Proc. Natl. Acad. Sci. U. S. A. 108, 16565-70 (2011).

24. Hulshof, F. F. B. et al. NanoTopoChip: High-throughput nanotopographical cell instruction. Acta Biomater. 62, 188-198 (2017).

25. Werner, M. et al. Surface Curvature Differentially Regulates Stem Cell Migration and Differentiation via Altered Attachment Morphology and Nuclear Deformation. Adv. Sci. 4, 1-11 (2017).

26. Hook, A. L. et al. High throughput methods applied in biomaterial development and discovery. Biomaterials 31, 187-198 (2010).

27. Yliperttula, M., Chung, B. G., Navaladi, A., Manbachi, A. \& Urtti, A. High-throughput screening of cell responses to biomaterials. European Journal of Pharmaceutical Sciences (2008). doi:10.1016/j.ejps.2008.04.012

28. van der Boon, T. A. B. et al. Well Plate Integrated Topography Gradient Screening Technology for Studying Cell-Surface Topography Interactions. Adv. Biosyst. (2020). doi:10.1002/adbi.201900218

29. Dalby, M. J. et al. The control of human mesenchymal cell differentiation using nanoscale symmetry and disorder. Nat. Mater. 6, 997-1003 (2007).

30. Huang, J. et al. Impact of Order and Disorder in RGD Nanopatterns on Cell Adhesion. Nano Lett. 9, 1111-1116 (2009).

31. Spagnolo, B. et al. Three-dimensional cage-like microscaffolds for cell invasion studies. Sci. Rep. 5, 1-10 (2015).

32. Wang, Y. et al. Benchtop micromolding of polystyrene by soft lithography. Lab Chip (2011). doi:10.1039/c1lc20281b

33. van Loosdrecht, M. C. M., Norde, W., Lyklema, J. \& Zehnder, A. J. B. Hydrophobic and electrostatic parameters in bacterial adhesion. Aquat. Sci. (1990). doi:10.1007/bf00878244

34. Hung, O. S., Thiyagarajan, V. \& Qian, P. Y. Preferential attachment of barnacle larvae to natural multi-species biofilms: Does surface wettability matter? J. Exp. Mar. Bio. Ecol. (2008). doi:10.1016/j.jembe.2008.04.011

35. Cheng, Y., Feng, G. \& Moraru, C. I. Micro-and nanotopography sensitive bacterial attachment mechanisms: A review. Frontiers in Microbiology (2019). doi:10.3389/fmicb.2019.00191

36. Huang, J. \& Kunitake, T. Nano-precision replication of natural cellulosic substances by metal oxides. J. Am. Chem. Soc. (2003). doi:10.1021/ja037419k

37. Goral, V. N., Hsieh, Y. C., Petzold, O. N., Faris, R. A. \& Yuen, P. K. Hot embossing of plastic microfluidic devices using poly(dimethylsiloxane) molds. J. Micromechanics Microengineering 21, (2011). 38. McQuin, C. et al. CellProfiler 3.0: Next-generation image processing for biology. PLoS Biol. 16, 1-17 (2018).

39. Ermis, M., Antmen, E. \& Hasirci, V. Micro and Nanofabrication methods to control cellsubstrate interactions and cell behavior: A review from the tissue engineering perspective. Bioactive Materials (2018). doi:10.1016/j.bioactmat.2018.05.005

40. Biela, S. A., Su, Y., Spatz, J. P. \& Kemkemer, R. Different sensitivity of human endothelial cells, smooth muscle cells and fibroblasts to topography in the nano-micro range. Acta Biomater. (2009). doi:10.1016/j.actbio.2009.04.003

41. Bach Q. Le et al. Micro-Topographies Promote Late Chondrogenic Differentiation Markers in the ATDC5 Cell Line. Tissue Eng. Part A 23, 458-469 (2017).

42. Vermeulen, S. et al. Surface topography is a context-dependent activator of TGF- $\beta$ signaling in mesenchymal stem cells. BioRxiv (2020).

43. Arciola, C. R., An, Y. H., Campoccia, D., Donati, M. E. \& Montanaro, L. Etiology of implant orthopedic infections: A survey on 1027 clinical isolates. Int. J. Artif. Organs (2005). doi:10.1177/039139880502801106

44. Mittal, R., Aggarwal, S., Sharma, S., Chhibber, S. \& Harjai, K. Urinary tract infections caused by Pseudomonas aeruginosa: A minireview. Journal of Infection and Public Health (2009). doi:10.1016/j.jiph.2009.08.003 
45. Bitsori, M., Maraki, S., Koukouraki, S. \& Galanakis, E. Pseudomonas aeruginosa urinary tract infection in children: Risk factors and outcomes. J. Urol. (2012). doi:10.1016/j.juro.2011.09.035

46. Gaynes, R. \& Edwards, J. R. Overview of Nosocomial Infections Caused by Gram-Negative Bacilli. Healthc. Epidemiol. 41, 848-854 (2005).

47. Kurmoo, Y. et al. Real time monitoring of biofilm formation on coated medical devices for the reduction and interception of bacterial infections. Biomater. Sci. (2020). doi:10.1039/c9bm00875f

48. Marsh, L. K. Primate species at the Tiputini Biodiversity Station, Ecuador. Neotrop. Primates (2004). doi:10.1016/j.joca.2010.01.017

49. Bogaert, J., Ceulemans, R. \& Salvador-Van Eysenrode, D. Decision Tree Algorithm for Detection of Spatial Processes in Landscape Transformation. Environ. Manage. (2004). doi:10.1007/s00267-003-0027-0

50. Zook, D. Tropical rainforests as dynamic symbiospheres of life. Symbiosis (2010). doi:10.1007/s13199-010-0071-5

51. Blake, J. G. et al. Temporal activity patterns of terrestrial mammals in lowland rainforest of Eastern Ecuador. Ecotropica (2012).

52. Salbut, L., Pakuła, A., Tomczewski, S. \& Styk, A. Portable profilometer based on low-coherence interferometry and smart pixel camera. in Speckle 2010: Optical Metrology (2010). doi:10.1117/12.871532 53. Vasilevich, A. S., Carlier, A., de Boer, J. \& Singh, S. How Not To Drown in Data: A Guide for Biomaterial Engineers. Trends Biotechnol. 35, 743-755 (2017).

54. Vasilevich, A. \& de Boer, J. Robot-scientists will lead tomorrow's biomaterials discovery. Current Opinion in Biomedical Engineering (2018). doi:10.1016/j.cobme.2018.03.005

55. Aggour, K. S. et al. Artificial intelligence/machine learning in manufacturing and inspection: A GE perspective. MRS Bulletin (2019). doi:10.1557/mrs.2019.157

56. Zhao, Y. et al. High-definition micropatterning method for hard, stiff and brittle polymers. Mater. Sci. Eng. C 71, 558-564 (2017).

57. Schindelin, J. et al. Fiji - an Open platform for biological image analysis. Nat. Methods 9, 241 (2009).

58. Haralick, R. M., Dinstein, I. \& Shanmugam, K. Textural Features for Image Classification. IEEE Trans. Syst. Man Cybern. (1973). doi:10.1109/TSMC.1973.4309314 


\section{Supplementary Figures}



Calla lily

Zantedeschia aethiopica

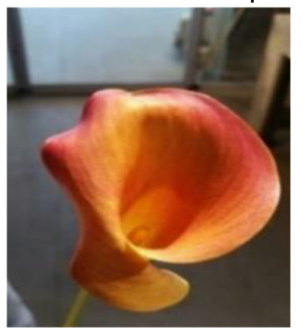

Sedum

Euphorbia sp.



Elephants ear Alocasia poly



Holy lotus Nelumbo nucifera

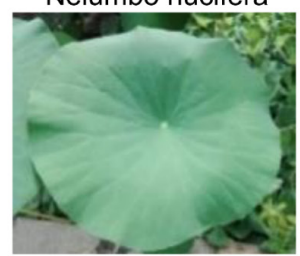

Hortensia

Hydrangea sp.

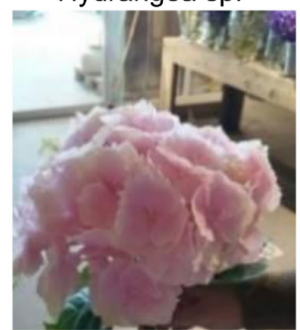

Reedmace

Typha latifola

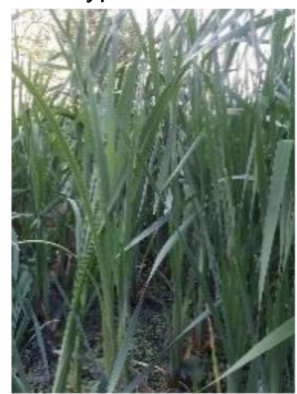

Heliconia

Heliconia psittacorum

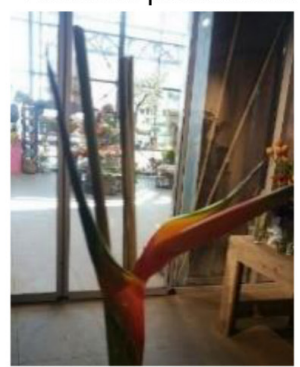

Rice

Oryza sativa



Red rose

Rosa rehd

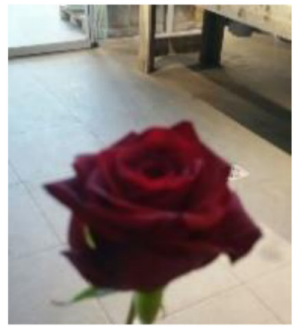

Onion

Allium sp.



Pineapple lily

Eucomis regia



Venus flytrap

Dionaea muscipula

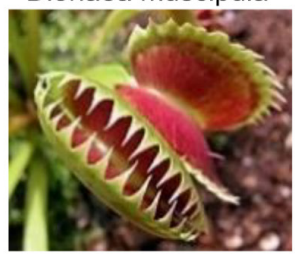

Curcuma

Curcuma longa

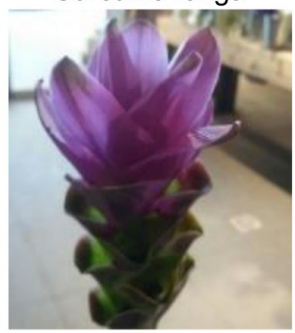

Hardy canna

Thalia dealbata

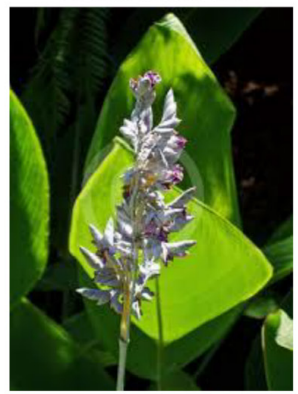

Laceleaf

Anthurium sp.



Supplementary Figure 1. Photographs of the sixteen plants used to obtain PS replicas of their leave's topography. 
Metallic wood boring beetle Shining leaf chafer beetle Megloxantha bicolor



Chrysophora chrysochlora

Cidada red

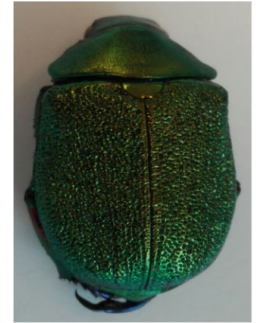

Gaeana festiva

Giant cicada

Quesada gigas



Yanga adriana

Yanga grandidieri


Cidada orange

Becquartina electa



Huechys incarnata

Leaf's insect

Gray's leaf

Phyllium westwoodii

Phyllium bioculatum
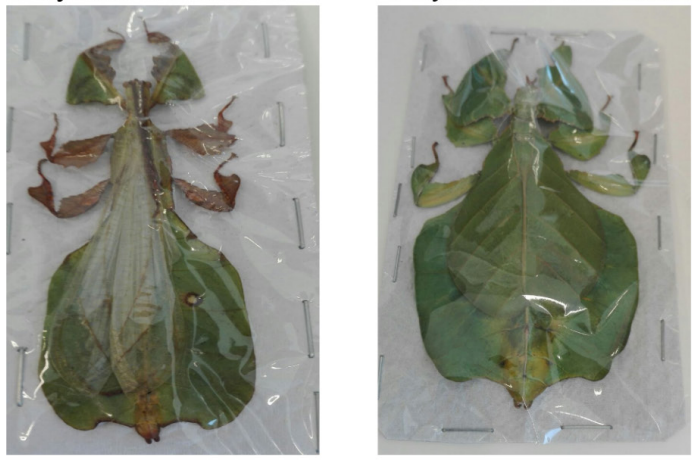

Supplementary Figure 2. Photographs of the ten insects used to obtain PS replicas of their wings. 
A



B

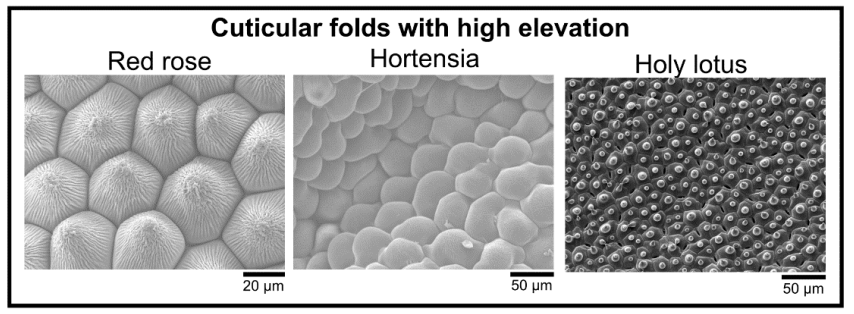

C


Supplementary Figure 3. SEM images of plant surfaces revealed a broad diversity in surface topography. A) Structures with low elevation are present on the Laceleaf, Calla lily, Curcuma, Sedum, Heliconia, Plaintain lily, and Hardy canna. B) Cuticular folds with complex and hierarchical structures are found on the Red rose, Hortensia, and Holy lotus. C) Oriented structures are found on the Onion, Reedmace, and Rice. D) Complex structures are found on the Pineapple lilly and Venus flytrap. E) Crystalline waxes present on the Elephants Ear give rise to micro-roughness structures. 
A


A



Supplementary Figure 4. SEM images of insect surfaces expanded the design space with nanotopographies, wells, and curvature. A) Nanopillars with different dimensions are found on different cicada species. B) Beetles exhibit pit-like structures on their surface, as shown for the Shining leaf chafer beetle and the metallic-wood boring beetle. C) Surfaces containing curvature are found on insects resembling leaves of plants, as shown for the Leaf's insect.
B

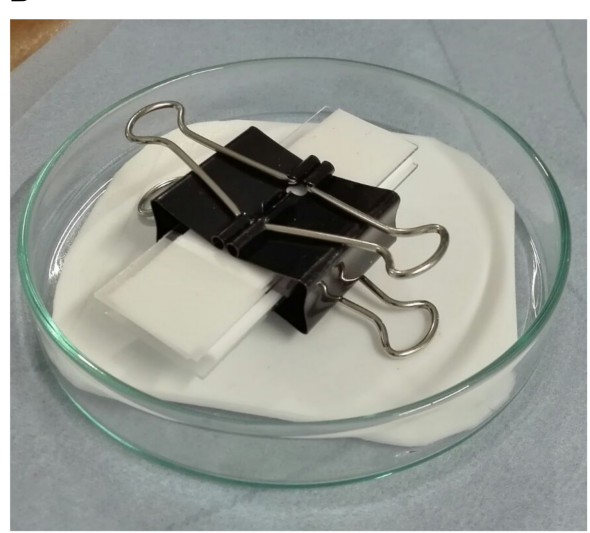

Supplementary Figure 5. Easy 'sandwich' imprinting fabrication. A) PDMS is poured on the Red rose imprint, after which it is peeled-off after curing for 24h. B) Afterwards, Teflon sheets, PDMS, and PS are sandwiched and pressured together with binders before placing the 'sandwich' in the oven. 
A



B



Supplementary Figure 6: The Red rose topography can be transferred with high fidelity to polystyrene. SEM images of the red rose polystyrene imprint at A) low, and B) high magnification.

A

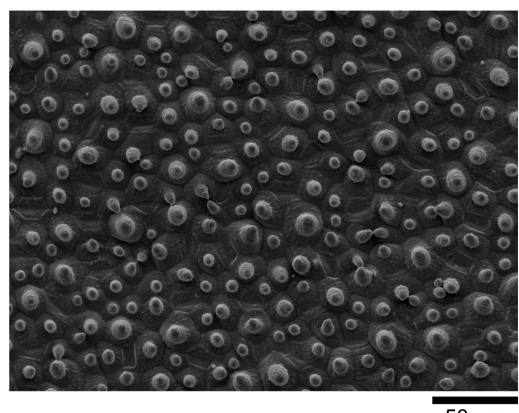

B

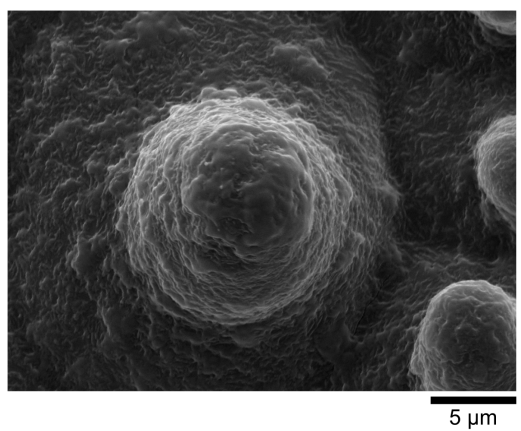

Supplementary Figure 7: The Holy Lotus topography can be transferred with high fidelity to polystyrene. SEM images of Holy Lotus polystyrene imprint at A) low, and $\mathbf{B}$ ) high magnification.

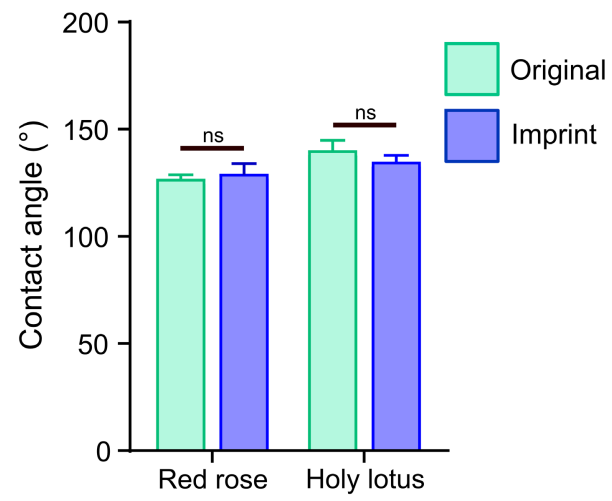

Supplementary Figure 8: No difference in the water contact angle was seen between the original surface and the PS imprints of red rose and Holy Lotus. 


\section{Chapter IX - Discussion}

\section{Modulation of biochemical signaling}

through shaping the cells mechanical environment

Steven Vermeulen ${ }^{1,2}$ and Jan de Boer ${ }^{2}$

${ }^{1}$ Laboratory for Cell Biology-Inspired Tissue Engineering, MERLN Institute, University of Maastricht, Maastricht, The Netherlands

${ }^{2}$ BioInterface Science Group, Department of Biomedical Engineering and Institute for Complex Molecular Systems, University of Eindhoven, Eindhoven, The Netherlands.

Manuscript in preparation 


\section{Cells undergo phenotypical changes in vitro}

Standard cell culture conditions comprise gas plasma etched polystyrene as substrate, a $21 \%$ atmospheric oxygen environment, and cell culture media, which is supplemented with $10 \%$ fetal bovine serum, a situation that is only sparsely recapitulating the native conditions found in normal physiology. Therefore, primary cells in culture undergo rapid changes in phenotype and often lose expression of phenotypical markers. For instance, tenocytes, the primary cell type of the tendon, already fail to keep phenotypical characteristics such as their spindle-shaped morphology and tenogenic marker expression after a few passages in standard cell culture conditions 1,2. Similar observations are reported for chondrocytes 3, cardiomyocytes 4, hepatocytes ${ }^{5}$, and MSCs, the latter of which lose their multipotency ${ }^{6}$. Similarly, without an appropriate feeder layer or media composition, embryonic stem cells (ESCs) will lose their pluripotency ${ }^{7}$. However, the fact that we can culture ES cells and iPSCs in vitro indefinitely is proof of principle for the concept that it is possible to define cell culture conditions that can sustain cell phenotypes. Significant focus has been put on defining the chemical composition of media, i.e. supplementation of media with small molecules and proteins, to provide the cells with the right signals. Furthermore, cell lines such as the neuronal-like cell line PC12 require a collagen or laminin coating as substrate to attach and proliferate ${ }^{8}$ due to the expression of $\alpha_{1} \beta_{1}$ and $\alpha_{3} \beta_{1}$ integrins that bind to these matrix proteins ${ }^{9}$. Fibronectin, one of the main proteins present in FBS, does not promote PC12 cell attachment ${ }^{10}$. It illustrates the critical role of cellsubstrate properties for culturing cells, but how do we select the right set of signals for the phenotypic maintenance of our favorite cell type?

\section{Lessons learned from developmental biology and tissue physiology}

Cells receive a wide variety of signals from their environment, both from soluble and matrixrelated cues. For example, peptide sequences found in matrix proteins such as fibronectin, collagen, and laminin, interact with integrin proteins of the cell, which lead to activation of signaling pathways ${ }^{11,12}$. Embryonic stem cell behavior can, as such, be modulated by coating culture substrates with matrix proteins that either guide differentiation or promote pluripotency 13. Soluble cues, such as growth factors and cytokines, modulate cell signaling by binding to receptor molecules on the cell membrane and is an essential means of communication. This signaling is transmitted either through autocrine signaling, where the secreted biochemical binds to receptors of the same cell or through paracrine signaling where the biochemical binds to receptors of other cells. These concepts occur in a multitude of cellular events. For example, vascular endothelial growth factor (VEGF) is secreted by cancer cells that, through autocrine signaling, activate the VEGF receptor (VEGFR), thereby establishing a strong auto feedback loop stimulating their growth and survival ${ }^{14}$. Furthermore, VEGF also elicits paracrine signaling in endothelial cells, which results in the vascularization of the tumor tissue ${ }^{15}$. Understanding these signaling pathways is essential for developing novel therapeutics. Also, in the adult 
organism, paracrine signaling is an important strategy for tissue homeostasis, as seen in mesenchymal stem cells (MSCs) that secrete immunomodulatory, anti-apoptotic, and proangiogenic growth factors ${ }^{16}$.

In addition to biochemical signaling, mechanical cues trigger cellular signaling events and are essential for proper tissue maintenance and developmental processes. Already before embryogenesis, an actin spring generates pressure, which allows a sperm cell to penetrate and activate the egg cell ${ }^{17}$. Hydrostatic pressure through osmosis triggers the activation of oocytes through $\mathrm{Ca}^{2+}$ signaling, which is related to cell volume changes when the cell passes into narrow oviducts ${ }^{18}$. Cell volume through osmosis can be applied in the culture dish as well because it affects the differentiation potential of MSCs ${ }^{19}$. Tissue organization during embryonic gastrulation uses actomyosin-dependent cell-cortex mechanical tension ${ }^{20}$. The first heartbeats in the embryo exert biomechanical forces in the form of shear stress and are responsible for the development of the cardiovascular system ${ }^{21}$. Also, the interaction between the cell and the mechanical properties of the surrounding environment profoundly affects cell behavior. This is demonstrated in experiments where low stiffness levels, such as in the human brain, facilitates the differentiation of MSCs towards neurons, while a harder environment promotes osteogenesis ${ }^{22}$, in concordance with the high stiffness of bone tissue. Furthermore, stretching promotes matrix formation in tendon tissue ${ }^{23}$ and can facilitate the differentiation of MSCs towards mechanosensitive cells such as tenocytes ${ }^{24}$. The response is often mediated via mechanoresponsive signaling pathways, of which the YAP pathway is the most well-known ${ }^{25,26}$. These examples demonstrate that similar to growth factors, mechanical stimulation is crucial for proper development and tissue homeostasis.

A fascinating interplay occurs between pathways that govern biomechanical and biochemical signaling, which allows fine-tuned physiological responses. Myocytes, the primary cell type of the muscle, secretes insulin-like growth factor (IGF) upon mechanical stimulation, which leads to autocrine activation of muscle growth ${ }^{27}$. For proper tendon homeostasis, both mechanical stimulation and TGF- $\beta$ signaling are required ${ }^{28,29}$. Wound healing is mediated through the differentiation of fibroblasts towards myofibroblasts. This process is regulated both through the mechanical properties of the surrounding tissue ${ }^{30}$ and TGF- $\beta$ signaling ${ }^{31}$, with evidence existing of a modulatory effect on TGF- $\beta$ signaling through these mechanical properties ${ }^{32}$. Also, during embryonic development, interactions occur between physical and biochemical cues. An interesting example is seen during gastruloid self-organization, where the response to TGF- $\beta$ ligands depends on cell polarization, which is associated with the cellular localization of receptors on the membrane ${ }^{33}$. During follicle growth, aggregation of dermal progenitors through contractility-driven cellular pulling triggers the activation of $\beta$-catenin, which induces the expression and secretion of BMP2, leading to paracrine signaling in the adjacent tissue ${ }^{34}$.

These observations are achieved due to significant cross-talk between biomechanical pathways and those activated by soluble cues. This can occur due to direct physical interaction between components of both pathways. TAZ, for instance, influences TGF- $\beta$ signaling through controlling the nucleocytoplasmic shuttling of SMAD and recruiting them to TGF- $\beta$ responsive 
elements in the promoter ${ }^{35}$. This interaction is essential for ESC self-renewal, and without the presence of TAZ, TGF- $\beta$ signaling is inhibited. FOS, a protein that is upregulated upon mechanical stimulation ${ }^{36}$, can, together with JUN form complexes with SMAD proteins, to regulate transcription ${ }^{37}$. Evidence exists that Wnt can be regulated biomechanically, for example, through YAP/TAZ associated pathways ${ }^{38}$. This can be achieved through other mechanisms besides forming transcriptional complexes, such as through the inhibition of $\beta$-catenin phosphorylation elicited by TAZ. Of interest is that Wnt signaling influences growth factor related signaling during development and for maintaining tissue homeostasis. This cross-talk can be quite divers as shown for Wnt and BMP signaling. Depending on the cell-type and timing during development, synergistic, or antagonistic effects of the two pathways on gene expression levels and phenotypes are possible ${ }^{39}$. Again, this cross-talk can be achieved through physical interactions between pathways, for example, through Wnt/GSK-3 $\beta$ that influences the phosphorylation of SMAD1 and subsequent activity ${ }^{40}$. Another example of the convergence of such signaling pathways is found between BMP and Notch signaling, of which the latter is associated with cell-cell interactions. Here, activation of both pathways can lead to synergistic effects on target gene expression levels ${ }^{41}$.

Integrin receptors control cell-matrix interactions and thereby play important roles in cell signaling shaped by the mechanical properties of the environment. Of interest is that also here, cross-talk exists between integrins and signaling pathways elicited by soluble cues. For example, when incorporating VEGF on a matrix, a more robust response is initiated in endothelial cells compared to soluble VEGF alone ${ }^{42}$. This is due to the association of the VEGF receptor with $\beta 1$ integrins, which was improved through membrane receptor clustering, allowing a prolonged internalization of VEGFR2. In this context, other interactions exist between integrins and receptors. For example, the interaction between $\alpha \mathrm{v} \beta 3$ integrin and the PDGF $\beta$ receptor improves the biological activity of PDGF $\beta{ }^{43}$. Insulin-like growth factor receptor (IGFR) activity is modulated through $\beta 1$ integrins ${ }^{44}$, while $\alpha 5 \mathrm{v} 1$ integrins enhance EGF signaling ${ }^{45}$. Signaling by FGF-2 requires co-binding between $\alpha_{\mathrm{v}} \beta 3$ and FGFR for inducing proliferation in endothelial cells ${ }^{46}$. The cross-talk between growth factors and integrins might also function through the upregulation of integrins due to growth factor signaling itself. For example, hepatocyte growth factor (HGF) upregulates $\alpha 2 \beta 1$ integrins in kidney cells leading to different matrix binding affinities ${ }^{47}$. Also, growth factor and integrin signaling can be activated independently from each other, and lead to pathway conversion inducing synergistic effects in gene expression. An example is found for $\alpha 6 \beta 4$ integrin, which affects VEGF expression through the PI3K/Akt pathway ${ }^{48}$. VEGF, however, is also regulated by HIF1- $\alpha$ expression and epithelialmesenchymal-transmission. Synergistic effects on VEGF expression are thereby achievable in this pathway architecture when two or more of the upstream affecters are activated.

Understanding these concepts is important for tissue-engineering purposes. For example, the differentiation of ESCs or iPSCs towards primary cells will follow the same in vivo developmental stages, while phenotypical maintenance of cell types require similar signaling events as found in normal physiology. Could, therefore, applying both biomechanical and biochemical concepts in 
the culture dish, guide stem cells through the same signaling programs found in the embryo, and thereby improve differentiation?

\section{Biomaterials to engineer growth factor signaling}

In this thesis, we investigated how topographies influence the phenotype of tenocytes, describe mechanobiological pathways and epigenetic alterations elicited by them, and demonstrate that topographies from natural origin can further expand the topographical design space. However, the work described in this thesis which is likely to find the most impact in a tissue-engineering context, and warrants follow-up studies, is how micro-topographies influence other signaling pathways such as TGF- $\beta$ signaling. We observed an upregulation of the TGF- $\beta$ receptor II (TGF$\beta R-I I)$ in MSC grown on micro-topographies and demonstrated that this leads to improved TGF$\beta$ signaling. Interestingly, we have unpublished data showing an upregulation of the plateletderived growth factor receptor (PDGFR), members of the tumor necrosis factor receptor superfamily (TNFRS), and of ERBB receptor feedback inhibitors (ERRFI1), of which the latter belongs to a family of tyrosine receptor kinases. Furthermore, a downregulation is observed for proteins that interact with the thyroid hormone receptor. We also noted topography-dependent upregulation of different integrins such as integrin alpha 2 (ITGA2), integrin alpha 10 (ITGA10), integrin alpha 11 (ITGA11), integrin beta 1 (ITGB1), and integrin beta 2 (ITGB2). The increased presence of these integrins might influence growth factor-related signaling due to altered binding dynamics with growth factor receptors. In addition to differential regulation of receptors or proteins that interact with them, we also observe an upregulation of nerve growth factor (NGF) expression, an observation that might prove useful for MSC co-culture applications with neurons. We previously also demonstrated through multiplex ELISA that the secretome profile of MSCs strongly correlates to the specific micro-topography on which it is cultured ${ }^{49}$. All these observations indicate that micro-topographies might influence cell physiology through pathways that modify the activity of growth factor and cytokine signaling. We hypothesize that this effect of mechanotransduction on growth factor-related signaling is not only limited to microtopographies, but other physical cues as well. In this context, in-depth investigations in online repositories such as cBiT that contain microarray and RNA sequencing data of cells grown on biomaterials ${ }^{50}$, can provide researchers with a wealth of clues on how physical cues influence biological pathways.

Based on literature, we know that biomaterials influence a wide variety of mechanotransduction pathways that might influence growth factor related signaling. We previously mentioned that cotranscription factors YAP and TAZ are involved in mechanosensitive signaling to control organ growth and proliferation ${ }^{51,52}$. Stretching forces influence signaling through YAP translocation into the nucleus ${ }^{53}$, similar to physical cues such as adhesive islands ${ }^{54}$, the stiffness of the environment 55, and grooves in nano dimensions that translocate TAZ 56. Alterations in YAP/TAZ signaling can be tightly associated with alterations in actin dynamics. Related to this, the transcriptional activity of serum response factor (SRF) is modified through altered actin dynamics that affect its coactivator Myocardin Related Transcription Factor A (MRTFA) ${ }^{57}$. SRF 
is known to target a multitude of genes ${ }^{58}$, including $\alpha$-SMA ${ }^{59}$, which is also a TGF- $\beta$ target gene 60. Synergistic effects on $\alpha$-SMA expression are therefore possible through combining mechanical forces such as stretching with TGF- $\beta$. Furthermore, an interesting cross-talk is possible between the mechanosensitive transcription factors TAZ and MRTFA that fine-tune mechanical and growth factor signaling ${ }^{61}$. Wnt signaling, which we previously discussed to be related to growth factor signaling, is modulated through changing roughness levels of the substrate ${ }^{62}$, through spheroid formation by micro-wells ${ }^{63}$ and chitosan membranes ${ }^{64}$. Organoid formation through micro-wells is also known to influence Notch signaling ${ }^{65}$, of which the pathway influences growth factor and cytokine signaling in neural stem cells ${ }^{66}$. These examples demonstrate the diversity of how biomechanical stimulation initiates mechanosensitive pathways that influence growth factor related signaling.

Next, we expand the possibilities on how mechanotransduction, in particular by biomaterials, can influence growth factor related signaling and describe the resulting phenotypical consequences. We further apply a distinction in three types of biomechanical signaling events: 1) Modulation of the secretion profile of soluble cues that lead to autocrine or paracrine signaling. 2) Modulating the sensitivity of the cell against soluble cues through modifying receptor expression. 3) Modulating the cell's response against soluble cues by altering receptor activity through binding with other membrane proteins such as integrins and cadherins. In Figure 1, we provide the reader with an overview of the mechanisms discussed in this chapter. 


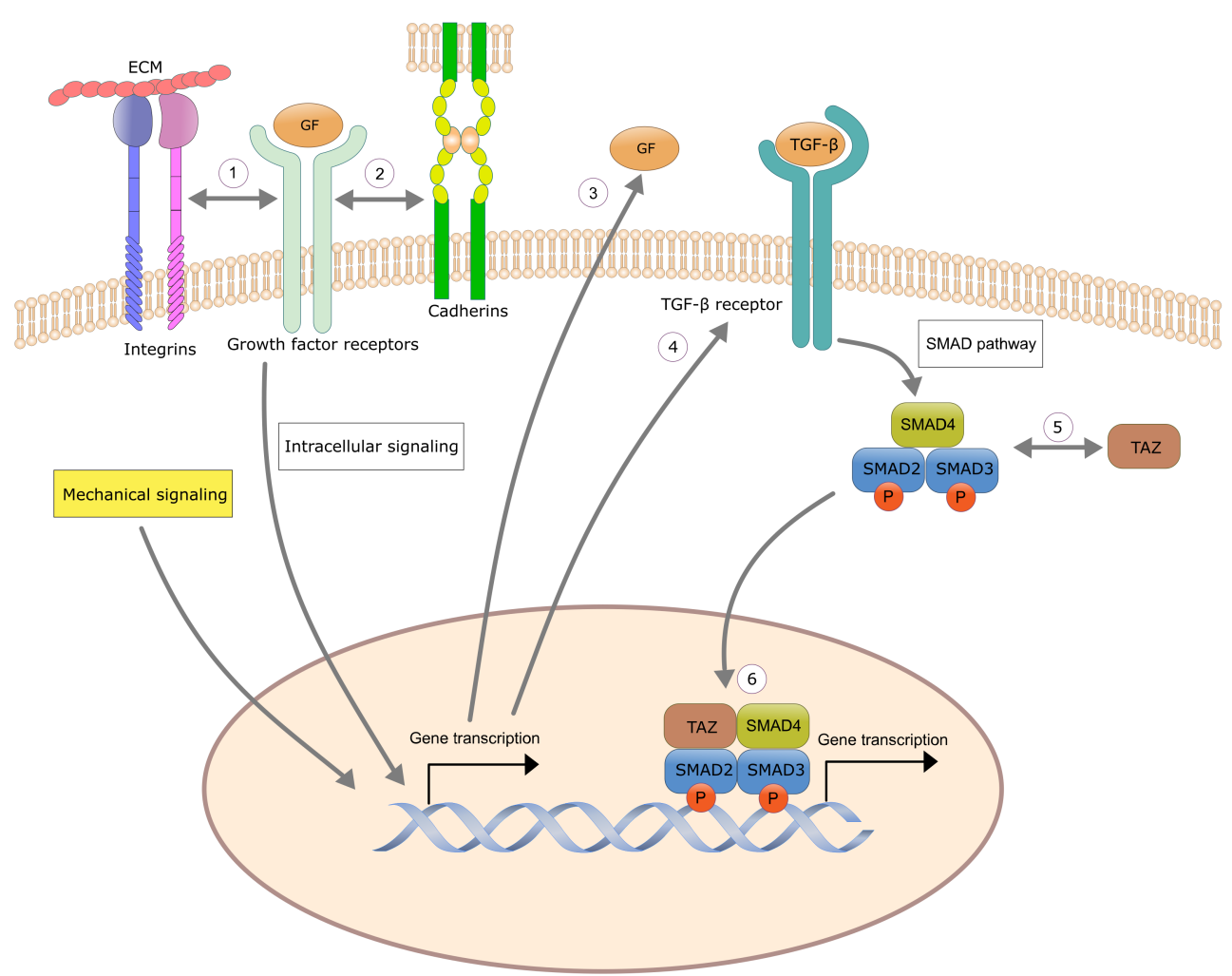

Figure 1: Overview of signal transduction mechanisms discussed in this chapter. 1) Integringrowth factor receptor cross-talk. 2) Cadherin-growth factor receptor cross-talk. 3) Modulation of the cells secretome profile due to mechanical stimuli. 4) Modulation of the cells sensitivity against growth factor signaling due to mechanical stimuli through alterations of growth factor receptor expression. 5) Interaction between mechanosensitive and growth factor related pathways. Depicted is TAZ that can interact with SMAD transcriptional complexes, which facilitates their nuclear transport. 6) Convergence between mechanosensitive and growth factor related pathways on transcriptional level. Depicted is the interaction between TAZ and SMAD proteins influencing their transcription.

\section{The mechanical environment determines the cells secretome profile}

We previously mentioned that mechanical stretching allows the secretion of IGF in myocytes, thereby stimulating their growth 27,67,68. The concept where mechanical stimulation elicits the secretion of growth factors can be mimicked in artificial environments as well. An example is found for MSCs, which upon stretching forces secrete different TGF- $\beta$ isoforms ${ }^{69}$, with a similar effect observed for tenocytes ${ }^{70}$. The secreted TGF- $\beta$ might be beneficial for inducing collagen synthesis, as shown for ligament cells ${ }^{71}$ and chondrocytes ${ }^{72}$. In vitro, it also improves differentiation protocols, as shown in a study that establishes a link between mechanical 
stimulation and TGF- $\beta$ secretion, which leads to autocrine signaling enhancing the differentiation of MSCs towards a tenogenic phenotype ${ }^{73}$.

Besides TGF- $\beta$, secretion of other growth factors through mechanical stretching is reported for satellite cells that secrete hepatocyte growth factor (HGF), thereby activating autocrine signaling events that lead to proliferation ${ }^{74}$. For aortic smooth muscle cells, hepatoma-derived growth factor (HDGF), which acts as a mitogen during embryogenesis and tissue repair, is secreted after mechanical stimulation ${ }^{75}$. Related to this, mechanical stimulation upregulates the cytokine IL-6 and downregulates IL-10 expression in epithelial cells mediated through the gene SOCS3, which can be a therapeutic target after lung injury by restoring the balance between pro-inflammatory and anti-inflammatory cytokines ${ }^{76}$. For vascular smooth muscle cells, mechanical stretching induces vascular endothelial (VEGF) and fibroblast growth factor (FGF) secretion, both involved in vascular development and angiogenesis ${ }^{77}$. We also report that chemokines such as SDF- $1 \alpha$ are secreted upon mechanical stimulation, thereby stimulating MSCs engaged in regenerating skin tissue ${ }^{78}$.

The deposition of growth factors in the tissue matrix might be regarded as indirect auto-or paracrine signaling when mechanical stimulation releases them and stimulates cells. Mechanically induced TGF- $\beta$ release can lead to fibrosis when the physical properties of the tissue have changed ${ }^{79}$. Similarly, physical forces are known to release TGF- $\beta$ from the tendon matrix, which, in physiological conditions, maintains the expression of Scleraxis, a tendon marker. However, upon destabilization of the ECM, excessive release of active TGF- $\beta$ leads can lead to tenocyte death ${ }^{80}$.

Besides mechanical stretching, physical cues such as the elasticity of the substrate elicits biomechanical signaling. Also here, multiple experiments demonstrate that introducing these in the cell culture landscape affects the secretion of soluble cues. For example, varying elasticity levels of hydrogels that mimic those of the brain or muscles elicits different secretome profiles in MSCs ${ }^{81}$. On a hard substrate, IL-8, a mediator in immune reactions, and VEGF were markedly upregulated compared to a soft substrate. This is a relevant finding for therapeutical purposes when MSCs are administered in vivo. The authors found that the cytoskeleton organization plays a vital role in this effect, as demonstrated by reduced cytoskeleton tension through the ROCK inhibitor Y27632 that abolished the observed effects. Cell shape and subsequent cytoskeleton organization are known to be profoundly affected by the elasticity levels of the substrate. The landmark paper of Disher illustrates this concept, where mimicking the tissue elasticity levels facilitates the differentiation of MSCs towards cell types of those tissues ${ }^{22}$. Of interest, through microarray profiling, an association between secreted soluble cues and elasticity was detected. Here, stiff elasticity levels were associated with osteogenic markers, while neurogenic markers were associated with the lowest elasticity levels. Reducing cytoskeletal tension through blebbistatin abolishes the effect of different elasticities. In another study, modulation of the MSC secretome through changing elasticity levels had beneficial effects on the proliferation of hematopoietic stem and progenitor cells (HSPCs), raising the possibility of using this concept for therapeutic applications such as HSPCs recovery ${ }^{82}$. 
Also other physical cues alter the cell's secretome. This is demonstrated by the TopoWell plate constructed in our group, where each well consists of micro-topographical structures in different dimensions. Through multiplex ELISA, we demonstrated a differential regulation of the secretome by these structures, both for bone marrow and kidney perivascular MSCs ${ }^{49}$. This is interesting for co-culture systems or therapeutical applications with these MSCs. For example, VEGF secretion was enhanced by specific structures, thereby raising the possibility to improve vascularization of tissue-engineering constructs when implementing these structures. In a similar approach, the multi-architecture chip (MARC), a plate-based screening platform with structures in both the micro- and nanodimensions, identified surface dimensions that either reduced or elevated IL-2 secretion in T-cells, with $200 \mathrm{~nm}$ gratings improving their proliferation potential 83. Also, different substrate roughness influences secretion, as shown for MG63 cells, which demonstrate elevated levels of TGF- $\beta 1$ and prostaglandin E1, which play essential roles in osteogenesis ${ }^{84}$. In this regard, nano-topographies enhance BMP-2 levels, thereby improving osteoblast differentiation through autocrine signaling ${ }^{85}$. Also coating scaffolds with calcium phosphate ceramics improves BMP-2 expression, although here it is uncertain if this caused by the topography or calcium/phosphate signaling from the materials ${ }^{86}$.

In a 3D environment, scaffolds with wavy patterns alter TGF- $\beta$ secretion in MSCs compared to scaffolds without patterns ${ }^{87}$. Similarly, hydrogels with different compositions can elicit secretome profile differences in multiple cell lines. Here, it was shown that alginate and PEG induce similar profiles, while eliciting different profiles compared to agarose and matrigel ${ }^{88}$. Micro-wells in small dimensions induce cellular confinement, which is associated with a reduced expression of inflammatory cytokines in macrophages ${ }^{89}$. Of interest, the authors related this to a reduction in SRF-MRTFA activity caused by reduced actin polymerization. Cells in a 3D environment, independent of the surrounding chemistry, can experience different growth factor signaling. This is demonstrated in a study where increased BMP autocrine signaling was observed compared to $2 \mathrm{D}$ when amniogenesis is initiated in iPSCs ${ }^{90}$. The biomechanical mechanisms behind these phenomena still need investigation. These examples demonstrate that the mechanical context plays essential roles in the secretion of soluble cues, which influence cell behavior in either an autocrine- or paracrine manner.

\section{The mechanical environment influences pathway sensitivity through modulating receptor expression}

Up- or downregulation of growth factor receptor expression is the second mechanism of action of biomaterial-induced modulation of biochemical signaling. For example, in normal physiology, retinal endothelial cells secrete more VEGF, yet also upregulate VEGF receptor expression after mechanical stimulation, a mechanism associated with hypertension ${ }^{91}$. Since VEGF plays a role in diabetic retinopathy, the observations might explain part of the clinical manifestations. Similarly, mechanical stimulation upregulates platelet-derived growth factor receptor (PDGF-R) in pulmonary artery-derived smooth muscle cells, reflecting mechanotransduction mechanisms occurring in the pulmonary artery ${ }^{92}$. For cells in the heart valve, which receive constant 
mechanical input, serotonin receptor (5HTR) expression depends on this signaling, relating biomechanics with proliferation and matrix deposition ${ }^{93}$. Here, ERK1/2 signaling was activated downstream of 5HTR.

Substrate elasticity also affects the cell's sensitivity against soluble cues. This was shown for microvascular endothelial cells, where elasticity upregulates the VEGF receptor, thereby sensitizing these cells for VEGF signaling and increasing their angiogenic potential ${ }^{94}$ through Rho signaling. Besides stretching and elasticity, beneficial effects on cell phenotype are possible when combining soluble cues with surface structures. Our group demonstrated this by adding TGF- $\beta 2$ in an MSC culture on micro-topographies ${ }^{95}$. Here, synergistic effects on TGF- $\beta$ sensitive genes are observed such as Scleraxis $(S C X)$ and $\alpha$-Smooth Muscle Cell Actin (a-SMA), which was linked to a higher expression of the TGF- $\beta$ Receptor II (TGF- $\beta$ R-II). Actin reorganization and Rho signaling events were crucial for the biomechanical signaling the microtopographies elicit, a phenomenon also associated with mechanical stretching ${ }^{96}$.

Similarly, as seen with the previous examples that switching from $2 \mathrm{D}$ to $3 \mathrm{D}$ influences secretion levels of growth factors, such an environment also influences receptor expression. This is shown for a sarcoma tumor cell line that in a scaffold environment upregulates insulin growth factor receptor (IGFR). The altered downstream signaling cascade makes these cells more resistant against clinical drug targets, thereby establishing a more realistic model for developing novel therapeutics ${ }^{97}$. Also inflammatory models are more realistic in a 3D environment as shown for spheroids that exhibited increased levels of lipopolysaccharide (LPS) receptors that modify the inflammatory response ${ }^{98}$. Receptors can also be downregulated as shown for TGF- $\beta$ receptors in a fibroblasts spheroid culture that exhibited decreased sensitivity against TGF- $\beta 1{ }^{99}$.

\section{Biomaterials mediate cross talk between membrane receptor proteins}

We previously mentioned that integrin expression is differentially regulated between cell types ${ }^{8,9}$, it is therefore a logical consequence that when designing a biomaterial this is factored into account. A material can have a high level of complexity to mimic a physiological environment, however without the presentation of appropriate binding sites, cells will be unable to attach. Furthermore, besides the observation that integrins on their own activate signaling cascades, the interaction between integrins and growth factor receptors is an important consideration in controlling cell behavior. This is suggested in a study where combinations of matrix molecules and growth factors attached in the matrix provides more beneficial effects compared to each single perturbation. In this case, improved neuronal stem cell differentiation is achieved through specific combinations of matrix proteins and growth factors ${ }^{100}$. In this regard, growth factors bound on matrix proteins such as fibronectin and hyaluronic acid allows an improvement in the clinical efficacy of wound healing ${ }^{101,102}$. Engineering VEGF binding domains in growth factors besides VEGF allows improved matrix binding such as in fibronectin. Through this, growth factors indeed improve their efficacy, facilitate receptor activation, and induce a more potent phenotypical response ${ }^{103}$. Research in the group of Jeff Hubble further explored this concept 206 
by utilizing ELISA assays to explore the affinity of different growth factors and their domains against different matrix proteins. Also here, engineering growth factors domains with high matrix affinities allows a stronger binding of these growth factors in the matrix, which leads to improved tissue healing. For example, bone healing could be improved through engineered BMP2 bound to the matrix ${ }^{104}$. Clinical trials involving BMP2 are often associated with off-targets effects due the large amounts that need to be administered ${ }^{105}$. Therefore, this approach is a promising alternative since less BMP2 is needed for achieving the most optimal clinical outcome. For a detailed discussion of the biology behind these phenomena and biomaterial applications that harness these concepts, we refer the reader to a recent review ${ }^{106}$.

Besides choosing appropriate matrix compositions, physical cues such as nano-topographies can also modify focal adhesion/integrin signaling ${ }^{107}$, which hints at a possibility to influence growth factor-related signaling events. This is demonstrated by a study documenting increased colocalization between $\beta 5$ integrin and the BMP receptor $1 \mathrm{a}$ through disordered nanotopographical patterns ${ }^{108}$. The co-localization was associated with increased sensitivity against BMP signaling, leading to improved osteogenesis. Another instance where topographical patterns influence receptor signaling is through calcium phosphate ceramics ${ }^{109}$. Culturing MSCs on this material allows improved TGF- $\beta$ receptor localization in the cilia, which exhibited an increased prevalence and length through the roughness levels of the substrate. This phenomena resulted in increased osteogenic differentiation in MSCs.

Besides integrins, we mention that other membrane proteins such as cadherins, which are cell adhesion molecules responsible for cell-to-cell binding, alter receptor activity through physical interaction. This is demonstrated for cadherin-catenin complexes with the EGF receptor, that modulating its signaling ${ }^{110}$. Also, a link exists between $\mathrm{N}$-cadherin and the FGF receptor, thereby raising the prospect of $\mathrm{N}$-cadherin being a therapeutic target against metastasis ${ }^{111}$. Vascular-endothelial cadherin attenuates VEGF receptor phosphorylation by VEGF ${ }^{112}$, a phenomenon also occurring between E-cadherin and the EGF receptor ${ }^{113,114}$. In ovarian cancer cells, P-cadherin cooperates with insulin-like growth factor receptor, thereby promoting metastasis through gonadotropin-releasing hormone ${ }^{115}$. Since cadherin activity is modulated through cell-cell adhesion ${ }^{116}$, these signaling regulation become important in confluent cell culture conditions. However, also in spheroids/organoids cultures that exhibit stronger cell-tocell binding due to their 3D environment, and which can be modulated by microwells ${ }^{117}$, this can be an important consideration to be taken into account. For example, in a spheroid culture of pancreatic tumor cells, it was shown that $\mathrm{N}$-cadherin and keratinocyte growth factor formed complexes that modulated tumor invasiveness ${ }^{118}$.

\section{Lessons learned from biomaterial science}

The concepts described in this discussion section are of great significance not only for the fields of tissue engineering or regenerative medicine. For novel drug discoveries against pathologies such as cancer or Alzheimer's disease, high-throughput screenings with advanced machine learning algorithms are becoming more the norm and can lead to exciting discoveries 
119. However, what if these screens are only able to capture a fraction of possible biological discoveries because the standard cell culture environment shape the expression of the repertoire of biochemical signaling proteins in a non-physiological way? To illustrate this, I demonstrated that micro-topographies alter the sensitivity against TGF- $\beta$ signaling ${ }^{95}$. Adhesive islands are known to modify the response of $3 \mathrm{~T} 3$ cells against $\mathrm{TNF} \alpha{ }^{120}$, while micro-wells alter inflammatory pathways in macrophages ${ }^{89}$. Considering these examples, it would not be surprising if many compounds lose their biological efficacy because cells are not put in a proper context that mimics physiological conditions.

By 2050, it is estimated that more than $20 \%$ of the world population will be older than 65 years 121. This global trend of an increased life expectancy is unfortunately associated with a rise in elderly-associated diseases, which are complex in origin. Commonly reported diseases are arthritis, diabetes, and brain diseases such as vascular dementia or Alzheimer's disease. This puts a tremendous burden on societal socio-economic infrastructure, emphasizing that there is an increased need for finding appropriate therapeutics. In this context, moving beyond the culture dish towards more appropriate physiological conditions can improve experimental outcomes. For example, in research performed prior to the work described in this thesis, I investigated the functional consequences of pathological mutations associated with Alzheimer's disease. In vitro assays can provide insight into the functional consequences of highly pathological mutations, such as for presenilin-1 and presenilin-2 mutations ${ }^{122}$. However, the reason why particular mutations are pathogenic might be more challenging to understand for risk factor genes such as Clusterin $(C L U)$, which only offer minor contributions to disease pathogenicity. When investigating six different CLU mutations that were associated with pathogenicity, for only three this could be coupled with a structural protein deficit ${ }^{123}$. Might it be that for these other mutations, their pathogenicity might become clear through applying physical cues, such as scaffolds or topographies?

\section{Conclusion}

In this chapter, we have demonstrated that both mechanical and biochemical cues interact at multiple levels both inside and outside the cell. This consideration is important for tissueengineering applications where we aim to understand and apply these processes. The function of growth factors and other soluble cues can be profoundly altered or improved when applying an appropriate mechanical environment, a consideration that is still easily neglected today. Especially for disease modelling and the search for novel therapeutical drugs, these concepts are of the utmost importance. In the future, the construction of more complicated and dynamic biomaterials that fully harness the interplay between biomechanical and chemical cues will help creating new opportunities for the tissue-engineering field, disease models, and personalized medicine. 


\section{References}

1. Vermeulen, S. et al. Identification of topographical architectures supporting the phenotype of rat tenocytes. Acta Biomater. 83, 277-290 (2019).

2. Yao, L., Bestwick, C. S., Bestwick, L., Maffulli, N. \& Aspden, R. M. Phenotypic drift in human tenocyte culture. Tissue Eng. 12, 1843-1849 (2006).

3. Holtzer, H., Abbot, J., Lash, J. \& S., H. The Loss of Phenotypic Traits by Differentiated Cells in vitro, I. Dedifferentiation of Cartilage Cells. PNAS 46, 1533-1542 (1960).

4. Bird, S. D. et al. The human adult cardiomyocyte phenotype. Cardiovasc. Res. 58, 423-434 (2003).

5. Kocarek, T., Schuetz, E. \& Guzelian, P. Expression of multiple forms of cytochrome P450 mRNAs in primary cultures of rat hepatocytes maintained on matrigel. Mol. Pharmacol. 43, 328-334 (1993).

6. Yang, Y. K., Ogando, C. R., See, C. W., Chang, T. \& Barabino, G. A. Changes in phenotype and differentiation potential of human mesenchymal stem cells aging in vitro. Stem Cell Res. Ther. 9, 131 (2018).

7. James A. Thomson, Joseph Itskovitz-Eldor, S. S. S., Michelle A. Waknitz, Jennifer J. Swiergiel, V. S. M. \& Jones, J. M. Embryonic Stem Cell Lines Derived from Human Blastocysts. Science (80-. ). 282, 1145-1147 (1998).

8. Tomaselli, K. J., Damsky, C. H. \& Reichardt, L. F. Interactions of a neuronal cell line (PC12) with laminin, collagen IV, and fibronectin: Identification of integrin-related glycoproteins involved in attachment and process outgrowth. J. Cell Biol. 105, 2347-2358 (1987).

9. Tomaselli, K. J. et al. A neuronal cell line (PC12) expresses two $\beta 1$-class integrins- $\alpha 1 \beta 1$, and $\alpha 3 \beta 1$ that recognize different neurite outgrowth-promoting domains in laminin. Neuron 5, 651-662 (1990).

10. Grinnell, F. \& Feld, M. K. Fibronectin adsorption on hydrophilic and hydrophobic surfaces detected by antibody binding and analyzed during cell adhesion in serum-containing medium. J. Biol. Chem. 257, 4888-4893 (1982).

11. Jokinen, J. et al. Integrin-mediated cell adhesion to type I collagen fibrils. J. Biol. Chem. 279, 31956-31963 (2004).

12. Belkin, A. M. \& Stepp, M. A. Integrins as receptors for laminins. Microsc. Res. Tech. 51, 280 301 (2000).

13. Hayashi, Y. et al. Integrins Regulate Mouse Embryonic Stem Cell Self-Renewal. Stem Cells 25, 3005-3015 (2007).

14. Weigand, M., Hantel, P., Kreienberg, R. \& Waltenberger, J. Autocrine vascular endothelial growth factor signalling in breast cancer. Evidence from cell lines and primary breast cancer cultures in vitro. Angiogenesis 8, 197-204 (2005).

15. Carmeliet, P. VEGF as a key mediator of angiogenesis in cancer. Oncology 69, 4-10 (2005).

16. Doorn, J., Moll, G., Le Blanc, K., van Blitterswijk, C. \& de Boer, J. Therapeutic Applications of Mesenchymal Stromal Cells: Paracrine Effects and Potential Improvements. Tissue Eng. Part B Rev. 18, 101-115 (2012).

17. Shin, J. H. et al. Force of an actin spring. Biophys. J. 92, 3729-3733 (2007).

18. Horner, V. L. \& Wolfner, M. F. Mechanical stimulation by osmotic and hydrostatic pressure activates Drosophila oocytes in vitro in a calcium-dependent manner. Dev. Biol. 316, 100-109 (2008).

19. Guo, M. et al. Cell volume change through water efflux impacts cell stiffness and stem cell fate. PNAS c, 1-10 (2017).

20. Krieg, M. et al. Tensile forces govern germ-layer organization in zebrafish. Nat. Cell Biol. 10, 429-436 (2008).

21. Adamo, L. et al. Biomechanical forces promote embryonic haematopoiesis. Nature 459, $1131-$ 1135 (2009).

22. Engler, A. J., Sen, S., Sweeney, H. L. \& Discher, D. E. Matrix Elasticity Directs Stem Cell Lineage Specification. Cell 126, 677-689 (2006).

23. Huisman, E., Lu, A., McCormack, R. G. \& Scott, A. Enhanced collagen type i synthesis by human tenocytes subjected to periodic in vitro mechanical stimulation. BMC Musculoskelet. Disord. 15, 1-8 (2014). 
24. Chen, X. et al. Force and scleraxis synergistically promote the commitment of human ES cells derived MSCs to tenocytes. Sci. Rep. 2, 977 (2012).

25. Halder, G., Dupont, S. \& Piccolo, S. Transduction of mechanical and cytoskeletal cues by YAP and TAZ. Nat. Rev. Mol. Cell Biol. 13, 591-600 (2012).

26. Panciera, T., Azzolin, L., Cordenonsi, M. \& Piccolo, S. Mechanobiology of YAP and TAZ in physiology and disease. Nat. Rev. Mol. Cell Biol. (2017). doi:10.1038/nrm.2017.87

27. Goldspink, D. F. et al. Muscle growth in response to mechanical stimuli. Am. J. Physiol. Endocrinol. Metab. 268, 288-297 (1995).

28. Rosager, S., Aagaard, P., Neergaard, K., Kjaer, M. \& Magnusson, S. P. Load-displacement properties of the human triceps surae aponeurosis and tendon in runners and non-runners. Scand. J. Med. Sci. Sport. 12, 90-98 (2002).

29. Potter, R. M. et al. Impact of TGF- $\beta$ inhibition during acute exercise on achilles tendon extracellular matrix. Am. J. Physiol. - Regul. Integr. Comp. Physiol. 312, R157-R164 (2017).

30. Kollmannsberger, P., Bidan, C. M., Dunlop, J. W. C., Fratzl, P. \& Vogel, V. Tensile forces drive a reversible fibroblast-to-myofibroblast transition during tissue growth in engineered clefts. Sci. Adv. 4, (2018).

31. Desmouliere, A., Geinoz, A., Gabbiani, F. \& Gabbiani, G. Transforming growth factor- $\beta 1$ induces $\alpha$-smooth muscle actin expression in granulation tissue myofibroblasts and in quiescent and growing cultured fibroblasts. J. Cell Biol. 122, 103-111 (1993).

32. Thannickal, V. J. et al. Myofibroblast differentiation by transforming growth factor- $\beta 1$ is dependent on cell adhesion and integrin signaling via focal adhesion kinase. J. Biol. Chem. 278, 12384 12389 (2003).

33. Etoc, F. et al. A Balance between Secreted Inhibitors and Edge Sensing Controls Gastruloid SelfOrganization. Dev. Cell 39, 302-315 (2016).

34. Shyer, A. E., Rodrigues, A. R., Schroeder, G. G., Kassianidou, E. \& Harland, R. M. Emergent Cellular Self-Organization and Mechanosensation Initiate Follicle Pattern in the Avian Skin. Science (80-. ). 357, 811-815 (2018).

35. Varelas, X. et al. TAZ controls Smad nucleocytoplasmic shuttling and regulates human embryonic stem-cell self-renewal. Nat. Cell Biol. 10, 837-848 (2008).

36. Eliasson, P., Andersson, T., Hammerman, M. \& Aspenberg, P. Primary gene response to mechanical loading in healing rat Achilles tendons. J. Appl. Physiol. 114, 1519-1526 (2013).

37. Zhang, Y., Feng, X.-H. \& Derynck, R. Smad3 and Smad4 cooperate with c-Jun/c-Fos to mediate TFG-b-induced transcription. Nature 394, 909-913 (1998).

38. Varelas, X. et al. The Hippo Pathway Regulates Wnt/ $\beta$-Catenin Signaling. Dev. Cell 18, 579-591 (2010).

39. Itasaki, N. \& Hoppler, S. Crosstalk between Wnt and bone morphogenic protein signaling: A turbulent relationship. Dev. Dyn. 239, 16-33 (2010).

40. Fuentealba, L. C. et al. Integrating Patterning Signals: Wnt/GSK3 Regulates the Duration of the BMP/Smad1 Signal. Cell 131, 980-993 (2007).

41. Takizawa, T., Ochiai, W., Nakashima, K. \& Taga, T. Enhanced gene activation by Notch and BMP signaling cross-talk. Nucleic Acids Res. 31, 5723-5731 (2003).

42. Chen, T. T. et al. Anchorage of VEGF to the extracellular matrix conveys differential signaling responses to endothelial cells. J. Cell Biol. 188, 595-609 (2010).

43. Schneller, M., Vuori, K. \& Ruoslahti, E. $\alpha \mathrm{v} \beta 3$ integrin associates with activated insulin and PDGF $\beta$ receptors and potentiates the biological activity of PDGF. EMBO J. 16, 5600-5607 (1997).

44. Goel, H. L. et al. Selective modulation of type 1 insulin-like growth factor receptor signaling and functions by $\beta 1$ integrins. J. Cell Biol. 166, 407-418 (2004).

45. Lee, J. W. \& Juliano, R. L. The $\alpha 5 \beta 1$ integrin selectively enhances epidermal growth factor signaling to the phosphatidylinositol-3-kinase/Akt pathway in intestinal epithelial cells. Biochim. Biophys. Acta - Mol. Cell Res. 1542, 23-31 (2002).

46. Sahni, A. \& Francis, C. W. Stimulation of endothelial cell proliferation by FGF-2 in the presence of fibrinogen requires aVb3. Blood 104, 3635-3641 (2004).

47. Chiu, S. J., Jiang, S. T., Wang, Y. K. \& Tang, M. J. Hepatocyte growth factor upregulates $\alpha 2 \beta 1$ integrin in Madin-Darby canine kidney cells: Implications in tubulogenesis. J. Biomed. Sci. 9, 261-272 (2002). 
48. Mercurio, A. M., Bachelder, R. E., Bates, R. C. \& Chung, J. Autocrine signaling in carcinoma: VEGF and the $\alpha 6 \beta 4$ integrin. Semin. Cancer Biol. 14, 115-122 (2004).

49. Leuning, D. G. et al. The cytokine secretion profile of mesenchymal stromal cells is determined by surface structure of the microenvironment. Sci. Rep. 8, 1-9 (2018).

50. Hebels, D. G. A. J., Carlier, A., Coonen, M. L. J., Theunissen, D. H. \& de Boer, J. cBiT: A transcriptomics database for innovative biomaterial engineering. Biomaterials 149, (2017).

51. Zhao, B. et al. Inactivation of YAP oncoprotein by the Hippo pathway is involved in cell contact inhibition and tissue growth control. Genes Dev. 21, 2747-2761 (2007).

52. Aragona, M. et al. A mechanical checkpoint controls multicellular growth through YAP/TAZ regulation by actin-processing factors. Cell 154, 1047-1059 (2013).

53. Elosegui-Artola, A. et al. Force Triggers YAP Nuclear Entry by Regulating Transport across Nuclear Pores. Cell 171, 1397-1410.e14 (2017).

54. Nardone, G. et al. YAP regulates cell mechanics by controlling focal adhesion assembly. Nat. Commun. 8, (2017).

55. Dupont, S. et al. Role of YAP / TAZ in mechanotransduction. Nature 474, 179-183 (2011).

56. Abagnale, G. et al. Surface Topography Guides Morphology and Spatial Patterning of Induced Pluripotent Stem Cell Colonies. Stem Cell Reports 9, 654-666 (2017).

57. Miralles, F., Posern, G., Zaromytidou, A. I. \& Treisman, R. Actin dynamics control SRF activity by regulation of its coactivator MAL. Cell 113, 329-342 (2003).

58. Miano, J. M., Long, X. \& Fujiwara, K. Serum response factor: Master regulator of the actin cytoskeleton and contractile apparatus. Am. J. Physiol. - Cell Physiol. 292, 70-81 (2007).

59. McDonald, O. G., Wamhoff, B. R., Hoofnagle, M. H. \& Owens, G. K. Control of SRF binding to $\mathrm{CArG}$ box chromatin regulates smooth muscle gene expression in vivo. J. Clin. Invest. 116, 36-48 (2006). 60. Hu, B., Wu, Z. \& Phan, S. H. Smad3 mediates transforming growth factor- $\beta$-induced $\alpha$-smooth muscle actin expression. Am. J. Respir. Cell Mol. Biol. 29, 397-404 (2003).

61. Speight, P., Kofler, M., Szászi, K. \& Kapus, A. Context-dependent switch in chemo/mechanotransduction via multilevel crosstalk among cytoskeleton-regulated MRTF and TAZ and TGF $\beta$-regulated Smad3. Nat. Commun. 7, (2016).

62. Abaricia, J. O., Shah, A. H., Chaubal, M., Hotchkiss, K. M. \& Olivares-Navarrete, R. Wnt signaling modulates macrophage polarization and is regulated by biomaterial surface properties. Biomaterials 243, (2020).

63. Azarin, S. M. et al. Modulation of Wnt/ $\beta$-catenin signaling in human embryonic stem cells using a 3-D microwell array. Biomaterials 33, 2041-2049 (2012).

64. Hsu, S. hui \& Huang, G. S. Substrate-dependent Wnt signaling in MSC differentiation within biomaterial-derived 3D spheroids. Biomaterials 34, 4725-4738 (2013).

65. Chen, K. et al. A Notch positive feedback in the intestinal stem cell niche is essential for stem cell self-renewal. Mol. Syst. Biol. 13, 927 (2017).

66. Nagao, M., Sugimori, M. \& Nakafuku, M. Cross Talk between Notch and Growth Factor/Cytokine Signaling Pathways in Neural Stem Cells. Mol. Cell. Biol. 27, 3982-3994 (2007).

67. Perrone, C. E., Fenwick-Smith, D. \& Vandenburgh, H. H. Collagen and Stretch Modulate Autocrine secretion of Insulin-like Growth Factor-1 and Insulin Growth Factor Binding Proteins from Differentiatied Skeletal Muscle Cells. J. Biol. Chem. 270, 2099-2106 (1995).

68. McKoy, G. et al. Expression of insulin growth factor-1 splice variants and structural genes in rabbit skeletal muscle induced by stretch and stimulation. J Physiol 516 ( Pt 2, 583-592 (1999).

69. Riser, B. L. et al. Cyclic stretching force selectively up-regulates transforming growth factor- $\beta$ isoforms in cultured rat mesangial cells. Am J Pathol 148, 1915-1923 (1996).

70. Skutek, M., Van Griensven, M., Zeichen, J., Brauer, N. \& Bosch, U. Cyclic mechanical stretching modulates secretion pattern of growth factors in human tendon fibroblasts. Eur. J. Appl. Physiol. 86, 4852 (2001).

71. Tetsuya Nakatani, Takashi Marui *, Toshiaki Hitora, Minoru Doita, Kotaro Nishida, M. K. Mechanical stretching force promotes collagen synthesis by cultured cells from human ligamentum flavum via transforming growth factor-b1. J. Orthop. Res. 20, 1380-1386 (2002).

72. Furumatsu, T. et al. Tensile strain increases expression of CCN2 and COL2A1 by activating TGF- $\beta-S m a d 2 / 3$ pathway in chondrocytic cells. J. Biomech. 46, 1508-1515 (2013). 
73. Grier, W. K., Moy, A. S. \& Harley, B. A. C. Cyclic tensile strain enhances human mesenchymal stem cell SMAD 2/3 activation and tenogenic differentiation in anisotropic collagen-glycosaminoglycan scaffolds. Eur. Cells Mater. 33, 227-239 (2017).

74. Koltzscher, M., Neumann, C., Kö, S. \& Gerke, V. Release of Hepatocyte Growth Factor from Mechanically Stretched Skeletal Muscle Satellite Cells and Role of pH and Nitric Oxide. Mol. Biol. Cell 13, 2909-2918 (2002).

75. Chen, P.-H. et al. Cyclic mechanical stretch up-regulates hepatoma-derived growth factor expression in cultured rat aortic smooth muscle cells. Biosci. Rep. 38, BSR20171398 (2018).

76. Hokenson, M. A. et al. Reduced IL-10 Production in Fetal Type II Epithelial Cells Exposed to Mechanical Stretch Is Mediated via Activation of IL-6-SOCS3 Signaling Pathway. PLoS One 8, (2013).

77. Quinn, T. P. et al. Cyclic mechanical stretch induces VEGF and FGF-2 expression in pulmonary vascular smooth muscle cells. Am J Physiol Lung Cell Mol Physiol 94118, 897-903 (2018).

78. Zhou, S. B., Wang, J., Chiang, C. A., Sheng, L. L. \& Li, Q. F. Mechanical stretch upregulates SDF- $1 \alpha$ in skin tissue and induces migration of circulating bone marrow-derived stem cells into the expanded skin. Stem Cells 31, 2703-2713 (2013).

79. Froese, A. R. et al. Stretch-induced activation of transforming growth factor-b1 in pulmonary fibrosis. Am. J. Respir. Crit. Care Med. 194, 84-96 (2016).

80. Maeda, T. et al. Conversion of Mechanical Force into TGF-b-Mediated Biochemical Signals. Curr. Biol. 21, 933-941 (2011).

81. Seib, F. P., Prewitz, M., Werner, C. \& Bornhäuser, M. Matrix elasticity regulates the secretory profile of human bone marrow-derived multipotent mesenchymal stromal cells (MSCs). Biochem. Biophys. Res. Commun. 389, 663-667 (2009).

82. Liu, F. D., Pishesha, N., Poon, Z., Kaushik, T. \& Van Vliet, K. J. Material Viscoelastic Properties Modulate the Mesenchymal Stem Cell Secretome for Applications in Hematopoietic Recovery. ACS Biomater. Sci. Eng. 3, 3292-3306 (2017).

83. Hu, J. et al. High-Throughput Mechanobiology Screening Platform Using Micro- and Nanotopography. Nano Lett. 55, 9557-9561 (2017).

84. Kieswetter, K. et al. Surface roughness modulates the local production of growth factors and cytokines by osteoblast-like MG-63 cells. J. Biomed. Mater. Res. 32, 55-63 (1996).

85. Castro-Raucci, L. M. S. et al. Titanium with Nanotopography Induces Osteoblast Differentiation by Regulating Endogenous Bone Morphogenetic Protein Expression and Signaling Pathway. J. Cell. Biochem. 117, 1718-1729 (2016).

86. Wang, J. et al. Nano-hydroxyapatite coating promotes porous calcium phosphate ceramicinduced osteogenesis via BMP/SMAD signaling pathway. Int. J. Nanomedicine 14, 7987-8000 (2019).

87. Chen, H. et al. From fiber curls to mesh waves: A platform for fabrication of hierarchically structured nanofibers mimicking natural tissue formation. Nanoscale 30, (2019).

88. Regier, M. C. et al. The Influence of Biomaterials on Cytokine Production in 3D Cultures. Biomacromolecules 18, 709-718 (2017).

89. Jain, N. \& Vogel, V. Spatial confinement downsizes the inflammatory response of macrophages. Nat. Mater. (2018). doi:10.1038/s41563-018-0190-6

90. Shao, Y. et al. Self-organized amniogenesis by human pluripotent stem cells in a biomimetic implantation-like niche. Nat. Mater. (2016). doi:10.1038/NMAT4829

91. Suzuma, I. et al. Cyclic stretch and hypertension induce retinal expression of vascular endothelial growth factor and vascular endothelial growth factor receptor-2: potential mechanisms for exacerbation of diabetic retinopathy by hypertension. Diabetes 50, 444-454 (2001).

92. Tanabe, Y. et al. Mechanical stretch augments PDGF receptor $\beta$ expression and protein tyrosine phosphorylation in pulmonary artery tissue and smooth muscle cells. Mol. Cell. Biochem. 215, 103-113 (2000).

93. Balachandran, K. et al. Aortic Valve Cyclic Stretch Causes Increased Remodeling Activity and Enhanced Serotonin Receptor Responsiveness Kartik. Ann Thorac Surg. 92, 147-153 (2011).

94. Mammoto, A. et al. A mechanosensitive transcriptional mechanism that controls angiogenesis. Nature 26, 1103-1108 (2009).

95. Vermeulen, S. et al. Surface topography is a context-dependent activator of TGF- $\beta$ signaling in mesenchymal stem cells. BioRxiv (2020). 
96. Asparuhova, M. B., Gelman, L. \& Chiquet, M. Role of the actin cytoskeleton in tuning cellular responses to external mechanical stress. Scand. J. Med. Sci. Sport. 19, 490-499 (2009).

97. Fong, E. L. S. et al. Modeling Ewing sarcoma tumors in vitro with 3D scaffolds. Proc. Natl. Acad. Sci. U. S. A. 110, 6500-6505 (2013).

98. Liu, J. et al. Three-dimensional spheroid cultures of A549 and HepG2 cells exhibit different lipopolysaccharide (LPS) receptor expression and LPS-induced cytokine response compared with monolayer cultures. Innate Immun. 17, 245-255 (2011).

99. Kunz-Schughart, L. A., Wenninger, S., Neumeier, T., Seidl, P. \& Knuechel, R. Three-dimensional tissue structure affects sensitivity of fibroblasts to TGF- 31 . Am. J. Physiol. - Cell Physiol. 284, 209-219 (2003).

100. Nakajima, M. et al. Combinatorial protein display for the cell-based screening of biomaterials that direct neural stem cell differentiation. Biomaterials 28, 1048-1060 (2007).

101. Xie, Y., Upton, Z., Richards, S., Rizzi, S. C. \& Leavesley, D. I. Hyaluronic acid: Evaluation as a potential delivery vehicle for vitronectin:growth factor complexes in wound healing applications. J. Control. Release 153, 225-232 (2011).

102. Upton, Z. et al. Human pilot studies reveal the potential of a vitronectin: Growth factor complex as a treatment for chronic wounds. Int. Wound J. 8, 522-532 (2011).

103. Wijelath, E. S. et al. Heparin-II Domain of Fibronectin Is a Vascular Endothelial Growth FactorBinding Domain. Circ. Res. 99, 853-860 (2006).

104. Martino, M. M. et al. Growth factors engineered for super-affinity to the extracellular matrix enhance tissue healing. Science (80-. ). 343, 885-888 (2014).

105. James, A. W. et al. A Review of the Clinical Side Effects of Bone Morphogenetic Protein-2. Tissue Eng. - Part B Rev. 22, 284-297 (2016).

106. Dalby, M. J. \& Salmeron-sanchez, M. Receptor control in mesenchymal stem cell engineering. Nat. Mater. 3, (2018).

107. Yim, E. K. F., Darling, E. M., Kulangara, K., Guilak, F. \& Leong, K. W. Nanotopographyinduced changes in focal adhesions, cytoskeletal organization, and mechanical properties of human mesenchymal stem cells. Biomaterials 31, 1299-1306 (2010).

108. Yang, J. et al. Nanotopographical Induction of Osteogenesis through Adhesion, Bone Morphogenic Protein Cosignaling, and Regulation of MicroRNAs. ACS Nano 8, 9941-53 (2014).

109. Zhang, J. et al. Topography of calcium phosphate ceramics regulates primary cilia length and TGF receptor recruitment associated with osteogenesis. Acta Biomater. 57, 487-497 (2017).

110. Hoschuetzky, H., Aberle, H. \& Kemler, R. $\beta$-Catenin mediates the interaction of the cadherincatenin complex with epidermal growth factor receptor. J. Cell Biol. 127, 1375-1380 (1994).

111. Suyama, K., Shapiro, I., Guttman, M. \& Hazan, R. B. A signaling pathway leading to metastasis is controlled by N-cadherin and the FGF receptor. Cancer Cell 2, 301-314 (2002).

112. Lampugnani, M. G. et al. Contact inhibition of VEGF-induced proliferation requires vascular endothelial cadherin, $\beta$-catenin, and the phosphatase DEP-1/CD148. J. Cell Biol. 161, 793-804 (2003).

113. Qian, X., Karpova, T., Sheppard, A. M., McNally, J. \& Lowy, D. R. E-cadherin-mediated adhesion inhibits ligand-dependent activation of diverse receptor tyrosine kinases. EMBO J. 23, 1739-1748 (2004).

114. Perrais, M., Chen, X., Perez-Moreno, M. \& Gumbiner, B. M. E-Cadherin Homophilic Ligation Inhibits Cell Growth and Epidermal Growth Factor Receptor Signaling Independently of Other Cell Interactions. Mol. Biol. Cell 18, 2013-2025 (2007).

115. Cheung, L. W. T. et al. P-cadherin cooperates with insulin-like growth factor-1 receptor to promote metastatic signaling of gonadotropin-releasing hormone in ovarian cancer via p120 catenin. Oncogene 30, 2964-2974 (2011).

116. Maître, J. L. \& Heisenberg, C. P. Three functions of cadherins in cell adhesion. Curr. Biol. 23, 626-633 (2013).

117. Fennema, E., Rivron, N., Rouwkema, J., van Blitterswijk, C. \& De Boer, J. Spheroid culture as a tool for creating 3D complex tissues. Trends in Biotechnology 31, 108-115 (2013).

118. Deramaudt, T. B. et al. N-Cadherin and Keratinocyte Growth Factor Receptor Mediate the Functional Interplay between Ki-RASG12V and p53V143A in Promoting Pancreatic Cell Migration, Invasion, and Tissue Architecture Disruption. Mol. Cell. Biol. 26, 4185-4200 (2006). 
119. Simm, J. et al. Repurposing High-Throughput Image Assays Enables Biological Activity Prediction for Drug Discovery. Cell Chem. Biol. 25, 611-618.e3 (2018).

120. Mitra, A. et al. Cell geometry dictates TNF $\alpha$-induced genome response. Proc. Natl. Acad. Sci. 114, E3882-E3891 (2017).

121. Ferruci, L., Giallauria, F. \& Guralnik, J. Epidemiology of Aging. Radiol Clin North am. 46, 1-19 (2009).

122. Samir Kumar-Singh, Jessie Theuns, C. V. B. Mean Age-of-Onset of Familal Alzheimers Disease Caused by Presenlin Mutations Correlates With Both Increased AB42 and Decresed AB40. Hum. Mutat. 27, 686-695 (2006).

123. Bettens, K. et al. Reduced secreted clusterin as a mechanism for Alzheimer-associated CLU mutations. Mol. Neurodegener. 10, 30 (2015). 


\section{Chapter X - Valorization}

Steven Vermeulen ${ }^{1,2}$ and Jan de Boer ${ }^{2}$

${ }^{1}$ Laboratory for Cell Biology-Inspired Tissue Engineering, MERLN Institute, University of Maastricht, Maastricht, The Netherlands

${ }^{2}$ BioInterface Science Group, Department of Biomedical Engineering and Institute for Complex Molecular Systems, University of Eindhoven, Eindhoven, The Netherlands. 


\section{Introduction}

In this chapter, we discuss the relevance of our research findings in a clinical/commercial context. Besides broadening our fundamental understanding of mechanobiology, an additional aim is to translate the scientific output into practice by exploring the commercialization possibilities of our research. This, we define as "Valorization". In Chapter 4, we discussed how the phenotype of tenocytes could be improved by culturing them on micro-topographies. In Chapter 5, we found that micro-topographies attenuate TGF- $\beta$ signaling in MSCs, which can lead to improved differentiation protocols. Furthermore, we found small molecules that mimic biomechanical signaling, which could be applied in a clinical setting. We also mention that the longer retainment of multipotency in MSCs described in Chapter 7 might be useful for harvesting secreted growth factors or creating appropriate co-culture conditions. In Chapter 8, we demonstrate that natural surface structures expand the design space of our regular microtopographies while eliciting distinct cell and bacterial behavior. In this Valorization Chapter, we therefore explore how these natural and artificial surface topographies can be commercialized.

\section{Social relevance of the research results and target audience}

The research results described in this thesis do not document if micro-topographies can be directly applied in a clinical setting. For example, would tenocytes cultured on microtopographies give rise to better tissue-engineering constructs for clinical applications compared to tenocytes cultured on regular polystyrene? Does a similar possibility exist for cells differentiated on micro-topographies? In addition, can coating natural and artificial topographies on medical implants inhibit bacterial infection? These questions are not answered in this thesis since clinical trials involving medical implants can take a significant amount of time ${ }^{1}$. Nevertheless, the results do show the potential of micro-topographies eventually being utilized in such clinical settings. Here, we would like to refer the reader to an article published in our group that demonstrated improved osteo-integration of titanium implants in rabbits when coated with micro-topographies ${ }^{2}$. Micro-topographies also allow guiding cell behavior, such as retaining the phenotype of cells or improving the differentiation of stem cells. This is not only supported by the research performed in this thesis, yet also by other research groups, which we discussed in Chapter 2 and 3. A possibility would be to harness micro-topographies to improve cell culture protocols in the culture dish for tissue-engineering purposes. For example, autologous stem cells can be harvested and cultured on micro-topographies for maintaining their phenotype for prolonged times. Work performed by Gilbert and co-workers nicely demonstrate this concept by utilizing hydrogels as a biomaterial ${ }^{3}$. In this work, muscle stem cells are isolated and put on an elastic hydrogel that promotes self-maintenance. This allowed growing them in sufficient cell numbers in vitro, after which implanting them in injured muscle improved tissue regeneration compared to cells cultured in standard conditions. If micro-topographies can play 
a similar role, with direct clinical applications, then this would increase their commercial and social value. However, additional research is required through animal models and eventually in humans, to assess the clinical feasibility of the surface structures utilized in this thesis. Therefore, we will mainly focus on describing the possibility of commercializing surface structures for research purposes.

\section{The product}

A key feature in commercializing surface structures for research purposes is creating a platform that offers the most optimal structural diversity, while ensuring the creation of phenotypical diversity. As described in Chapter 8, natural surface structures expand the design of our microtopographical library ${ }^{4}$, while creating novel phenotypical diversity. Therefore, when creating a novel platform, some of these natural surfaces should be included so that the design space is optimally expanded (Figure 1). However, despite the 2176 unique micro-topographies from the TopoChip and the natural surfaces explored in this thesis, such a platform would still fall short in representing true surface diversity. For example, grooves are not present in this design, which are known to induce specific and distinct phenotypes compared to our micro-topographies. For example, as shown in Chapter 7, micro-topographies lower histone acetylation levels, while micro-grooves are known to elevate them ${ }^{5}$. Furthermore, surface roughness, wells, or curvature are also not included. In addition, structures in nanodimensions ${ }^{6}$, a combination of both ${ }^{7}$, and disorder instead of ordered structures ${ }^{8}$, are not included.

As a product suitable for commercialization, we would like to present surfaces in a 24 -well format size to enable sufficient cell numbers for multiple experimental setups such as qPCR/microarray/RNAseq, western blot/ELISA, and ICC. Researchers can perform screens
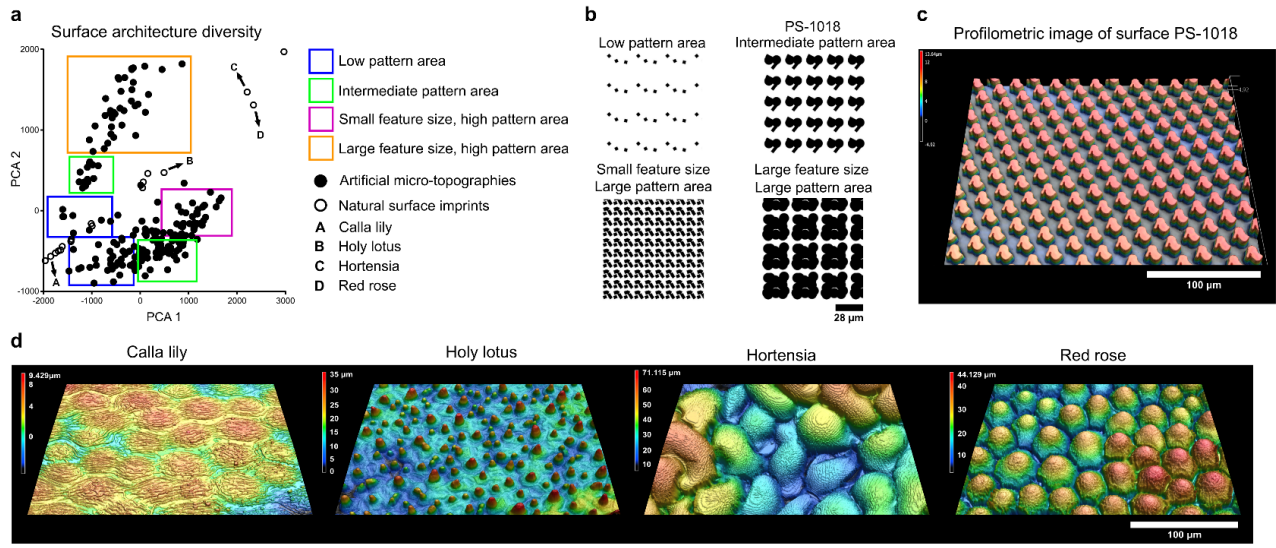

Figure 1: Figure from chapter 8 of this thesis, which illustrates how natural surface structures expand the design space compared to the micro-topographical library we commonly use. 
with this setup, after which it would be possible to order a single sheet of a surface structure in a $100 \mathrm{~mm}$ dish format for in-depth follow-up experiments. This can be made possible by constructing a silicone mould of each surface structure. Afterwards, polystyrene sheets are fabricated according to the method described in the work of Zhao et al. ${ }^{9}$. Microwells can be constructed through thermoforming techniques ${ }^{10}$, while for the natural surface structures a positive PDMS imprint can be generated through double PDMS casting ${ }^{11}$ instead of using a silicon mould as a starting material. An automatic laser cutter can be applied to cut a single PS sheets in suitable 24-well format dimensions.

Next to the classical PS material, also other materials can be offered such as polyurethane (PU) to enhance the biological readouts or to test surface structures that were fabricated with materials used in the clinic.

The proposed library would contain the following 22 unique structures, which are known to induce specific cellular phenotypes (Figure 2):

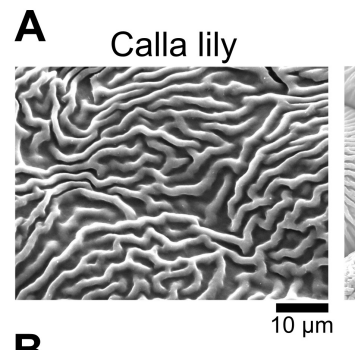

B



C

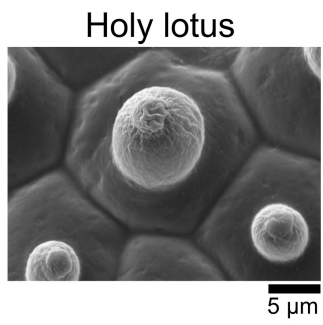

$5 \mu \mathrm{m}$


Figure 2: Sample of the distinct surfaces present on this new platform. A) SEM images of the natural surfaces. B) Roughness and groove patterns. C) Micro-wells in different dimensions.

1) Micro-topography - Small feature size, small pattern area (TopoChip library) 4

2) Micro-topography - Large feature size, large pattern area (TopoChip library) ${ }^{4}$

3) Micro-topography - Large feature size, small pattern area (TopoChip library) ${ }^{4}$

4) Nanotopographies - Large pattern area (Nano'TopoChip library) ${ }^{6}$

5) Nanotopographies - Small pattern area (Nano'TopoChip library) ${ }^{6}$

6) Calla lily - Complex structures with low elevation (Natural surface library) 
7) Red rose - Hierarchical micropillars with nanofoulds (Natural surface library)

8) Holy lotus - Hierarchical micropillars with surface roughness (Natural surface library)

9) Microgrooves - $1 \mu \mathrm{m}$ depth, $40 \mu \mathrm{m}$ wide 5

10) Microgrooves - $1 \mu \mathrm{m}$ depth, $20 \mu \mathrm{m}$ wide ${ }^{5}$

11) Microgrooves - $1 \mu \mathrm{m}$ depth, $10 \mu \mathrm{m}$ wide ${ }^{5}$

12) Nanogrooves - $200 \mathrm{~nm}$ wide and $100 \mathrm{~nm}$ deep nanochannels ${ }^{12}$

13) Hierarchical micro-nanogrooves - $10 \mu \mathrm{m}$ wide and $1 \mu \mathrm{m}$ deep channels in combination with $200 \mathrm{~nm}$ wide and $100 \mathrm{~nm}$ deep nanochannels ${ }^{12}$

14) Microwells/pits - $30 \mu \mathrm{m}$ diameter ${ }^{13}$

15) Microwells/pits - $400 \mu \mathrm{m}$ diameter ${ }^{13}$

16) Nanopits - 120-nm-diameter, 100-nm-deep nanopits (ordered) 8

17) Nanopits - 120-nm-diameter, 100 -nm-deep nanopits (disordered) ${ }^{8}$

18) Convex curvature - $x=1 / 175 \mu \mathrm{m}^{-1} 14$

19) Microroughness - $\mathrm{Sa}=2 \mu \mathrm{m}$

20) Nanoroughness - $\mathrm{Sa}=900 \mathrm{~nm}$

21) Nanoroughness $-\mathrm{Sa}=70 \mathrm{~nm}^{7}$

22) Hierarchical micro-nano roughness $-\mathrm{Sa}=900 \mathrm{~nm}+70 \mathrm{~nm}^{7}$

23) Flat $2 x$

\section{Innovation}

Offering scientist platforms to study mechanobiology or perform screens is not novel, yet demonstrates that also our platform has commercialization potential. An example of such a successful commercialization concept is the biotech company CYTOO. This company offers high-throughput screens including those in an adhesive island format. Our design would fill a surface structure niche in the market. Here, we emphasize that even though our design space is very broad, it still does not encompass potential designs that can have distinct phenotypical readouts. For example, podocytes have a unique morphology, and biomaterials that mimic this cellular shape in vitro induce beneficial phenotypical effects in this cell type ${ }^{15}$. Furthermore, not all micro- or nanogroove dimensions are covered in our setup, as demonstrated by the polyimide platform ${ }^{16}$. Therefore, it is essential that efforts are continued to improve the design space and the platform. In the future, if many novel and functional design spaces are discovered, a 96-well format can be offered in plates. We previously demonstrated this for the TopoWellPlate ${ }^{17}$, yet this platform only contained micro-topographies. Options for further improving the design space would be to apply basic functional assays such as cell morphology, focal adhesion staining, and differentiation experiments on novel natural surfaces. Furthermore, applying artificial intelligence, as recently demonstrated by our group, can predict novel surface designs with interesting functionality ${ }^{18}$.

Additional research will allow novel innovations such as the possibility to implement these structures for clinical applications such as for medical implants. Also in the culture dish, investigating how these structures can promote cell differentiation or phenotypical maintenance 
protocols will further add to its commercialization potential. If a particular topography indeed gives rise to an intriguing phenotypical readout, then a collaboration could be considered with other companies to create commercial kits. These kits can then contain an appropriate cell culture media supplemented with growth factors and a surface substrate of interest to obtain a cell phenotype. Such companies could be Stem Cell Technologies, Lonza, or Thermo Fisher.

\section{Schedule \& Implementation}

Since this platform is strongly associated with the possibility of performing fundamental research, a plausible format for this product would be to develop a spin-off company around the concept. This can be achieved through university funding and local research groups. Furthermore, equipment, technical, and biological expertise are available there enabling a quick realization of the product. This also ensures a limited risk in commercializing this product since the research value can also be translated into research publications. As such, additional funding should be able to be acquired through research grants.

\section{References}

1. Rising, J. P. \& Moscovitch, B. Characteristics of pivotal trials and FDA review of innovative devices. PLoS ONE 10, (2015).

2. Hulshof, F. F. B. et al. Mining for osteogenic surface topographies: In silico design to in vivo osseo-integration. Biomaterials 137, 49-60 (2017).

3. Gilbert, P. et al. Substrate elasticity regulates skeletal muscle stem cell self-renewal in culture. Science (80-. ). 329, 1078-1081 (2011).

4. Unadkat, H. V et al. An algorithm-based topographical biomaterials library to instruct cell fate. Proc. Natl. Acad. Sci. U. S. A. 108, 16565-70 (2011).

5. Downing, T. L. et al. Biophysical regulation of epigenetic state and cell reprogramming. Nat. Mater. 12, 1154-1162 (2013).

6. Hulshof, F. F. B. et al. NanoTopoChip: High-throughput nanotopographical cell instruction. Acta Biomater. 62, 188-198 (2017).

7. Jaggy, M. et al. Hierarchical Micro-Nano Surface Topography Promotes Long-Term Maintenance of Undifferentiated Mouse Embryonic Stem Cells. Nano Lett. 15, 7146-7154 (2015).

8. Dalby, M. J. et al. The control of human mesenchymal cell differentiation using nanoscale symmetry and disorder. Nat. Mater. 6, 997-1003 (2007).

9. Zhao, Y. et al. High-definition micropatterning method for hard, stiff and brittle polymers. Mater. Sci. Eng. C 71, 558-564 (2017).

10. Truckenmüller, R. et al. Thermoforming of film-based biomedical microdevices. Adv. Mater. 23, 1311-1329 (2011).

11. Gitlin, L., Schulze, P. \& Belder, D. Rapid replication of master structures by double casting with PDMS. Lab Chip 9, 3000-3002 (2009).

12. López-Bosque, M. J. et al. Fabrication of hierarchical micro-nanotopographies for cell attachment studies. Nanotechnology 24, (2013).

13. Jain, N. \& Vogel, V. Spatial confinement downsizes the inflammatory response of macrophages. Nat. Mater. (2018). doi:10.1038/s41563-018-0190-6

14. Werner, M. et al. Surface Curvature Differentially Regulates Stem Cell Migration and Differentiation via Altered Attachment Morphology and Nuclear Deformation. Adv. Sci. 4, 1-11 (2017).

15. Ron, A. et al. Cell shape information is transduced through tension-independent mechanisms. Nat. Commun. 8, (2017).

16. Abagnale, G. et al. Surface topography enhances differentiation of mesenchymal stem cells towards osteogenic and adipogenic lineages. Biomaterials 61, 316-326 (2015). 
17. Leuning, D. G. et al. The cytokine secretion profile of mesenchymal stromal cells is determined by surface structure of the microenvironment. Sci. Rep. 8, 1-9 (2018).

18. Vasilevich, A., Carlier, A., Winkler, D. A., Singh, S. \& Boer, J. De. Evolutionary design of optimal surface topographies for biomaterials. bioRxiv (2020) 


\section{Summary}

This thesis investigates how physical cues influence cell behavior in the culture dish. Many surface structures exist, which complicates the identification of an optimal physical environment for a specific cellular phenotype. Therefore, high-throughput platforms enable the screening of multiple surface designs to find an optimal cell culture environment for a specific phenotype. By utilizing the TopoChip, which contains 2176 unique micro-topographies, we identified structures that support the phenotype of tenocytes, the primary cell type of the tendon. Similarly, we also found this to be true for mesenchymal stem cells (MSCs), which experience an activation of tendon-related genes upon contact with certain micro-topographies. This allows microtopographies to be considered for the differentiation of MSCs towards tenocytes. Of interest, we found these findings related to a sensitization of the TGF- $\beta$ signaling pathway. Furthermore, microarray analysis of MSCs grown on micro-topographies allowed us to gain insights into the biomechanical pathways that are activated. This led us to identify small molecules that mimic the effect of micro-topographies on TGF- $\beta$ signaling. The implication of this might be that biomechanical stimuli can be translated in a soluble form for clinical applications as well. Similar as to micro-topographies, actin organization was also found to be a driving factor in eliciting the expression of SRF and EGR-1 on surfaces where RGD presentation was varied.

Next to determining that micro-topographies influence TGF- $\beta$ signaling and a tenogenic phenotype, we found that the nuclear confinement elicited by micro-topographies coincides with epigenetic and nucleoli alterations. These findings were associated with a quiescent-like phenotype, as evidenced by a reduction in proliferation and a prolonged multipotency.

Although micro-topographies demonstrate their usefulness for implementation in a tissue culture environment, these structures only encompass a fraction of the total design space possibilities. We, therefore, chose to expand the topographical design space of the TopoChip by utilizing natural surface architectures. Of interest, these structures elicit a distinct phenotype in MSCs compared to MSCs grown on regular micro-topographies. In the future, designing platforms that further expand the design space will be of great interest for novel research and commercialization opportunities. 


\section{Samenvatting}

Dit proefschrift onderzoekt hoe oppervlaktestructuren het cel gedrag in de kweekschotel beïnvloeden. Er bestaat een grote diversiteit aan oppervlaktestructuren, wat de identificatie van een optimale fysieke omgeving voor een specifiek cellulair fenotype bemoeilijkt. Daarom vergemakkelijken platforms die toelaten om veel verschillende ontwerpen tegelijkertijd te screenen het vinden van een optimale cultuuromgeving voor een bepaald fenotype. Door gebruik te maken van de TopoChip, die 2176 unieke microtopografieën bevat, hebben we structuren geïdentificeerd die het fenotype van tenocyten, het primaire celtype van de pees, ondersteunen. Bovendien vonden we dat dit ook gold voor mesenchymale stamcellen (MSCs), die een activering van pees gerelateerde genen ervaren bij contact met bepaalde microtopografieën. Hierdoor kunnen micro-topografieën worden overwogen voor de differentiatie van MSCs naar tenocyten. Een interessante bevinding was dat deze resultaten verband hielden met een sensibilisatie van de TGF- $\beta$-signaleringsroute. Door gebruik te maken van een microarray-analyse van MSCs die zijn gegroeid op micro-topografieën, heeft dit ons in staat gesteld inzicht te krijgen in de biomechanische signaal routes die worden geactiveerd. Dit bracht ons ertoe om biomoleculen te identificeren die het effect van microtopografieën op TGF$\beta$-signalering nabootsen. Een mogelijke implicatie hiervan is dat biomechanische stimuli ook in oplosbare vorm geactiveerd kan worden, wat interessant kan zijn voor klinische toepassingen. Zoals bij micro-topografieën bleek de organisatie van actine ook een drijvende factor te zijn bij het opwekken van de expressie van SRF en EGR-1 op oppervlakken met verschillende RGDpresentaties.

Naast het vaststellen dat microtopografieën TGF- $\beta$-signalering en dus een tenocyt fenotype beïnvloeden, ontdekten we dat de door microtopografie geïnduceerde verkleining van de nucleus samenvalt met epigenetische veranderingen en een verminderde aanwezigheid van nucleoli. Deze bevindingen werden geassocieerd met een afname van proliferatie en een langere multipotentie duur.

Hoewel microtopografieën hun nut aantonen om gebruikt te worden in een weefselkweekomgeving, omvatten deze structuren slechts een fractie van de alle ontwerpruimtemogelijkheden. We hebben er daarom voor gekozen om de topografische ontwerpruimte van de TopoChip uit te breiden door gebruik te maken van natuurlijke oppervlakte architecturen. Interessant is dat deze structuren ook bij MSCs een ander fenotype opwekken in vergelijking met MSCs die op reguliere microtopografieën worden gekweekt. In de toekomst zullen het ontwerpen van platforms die de ontwerpruimte verder uitbreiden, van groot belang zijn voor nieuwe onderzoeks- en commercialisatiemogelijkheden. 


\section{Scientific Activities}

\section{Experimental Articles in International Journals}

Romero M., Carlier A., Carrabelli A., Vasilevich A., Vermeulen S., Beijer N., Hook A., Papenburg B., Ghaemmaghami A., de Boer J., Alexander, M., Williams P. Micro-scale topographies instruct bacterial attachment to surfaces. Manuscript in preparation

Kocer G.*, Vermeulen S.*, Brinkmann J., Gamo L., Hebels D., Vasilevich A., van de Peppel J., Sankaran S., Cavatorta E., van Leeuwen J., de Boer J. and Jonkheijma P. The Covalent State of RGD Peptide Presentation Dictates the Early Transcriptome Profile of 3T3 Cells. Manuscript in preparation.

* These authors contributed equally

Vermeulen S., Van Puyvelde B., Bengtsson del Barrio L., van der Veer B., Dhaenens M., de Boer J. Epigenetic Regulation of Mesenchymal Stem Cells by Micro-Topographies. Manuscript in preparation.

Vermeulen S., Honig F., Vasilevich A., Roumans N., Romero M., Williams P., Carlier A., Uquillas J., de Boer J. Extending Biomaterial Surface Topographical Design Space through Natural Surface Reproduction. Submitted for publication

Eroumé K., Vasilevich A., Vermeulen S., de Boer J., Carlier A. On the influence of cell shape on dynamic diffusion-reaction polarization patterns. Submitted for publication

Vasilevich A., Vermeulen S., Kamphuis M., Roumans N., Eroume S., Hebels D., Rika R., Beijer N., Carlier A., Carpenter A., Singh S. and de Boer J. On the correlation between material-induced cell shape and phenotypical response of human mesenchymal stem cells. Submitted for publication

Burroughs L., Amer M., Vassey M., Koch B., Figueredo G., Mukonoweshuro B., Mikulskis P., Vasilevich A., Vermeulen S., Dryden I., Winkler D., Ghaemmaghami A., Rose F., de Boer J. Determining Immune-Instructive and Osteoinductive Material Chemistry and MicroTopography Combinations Using the ChemoTopoChip. Submitted for publication

Vermeulen S., Roumans N., Honig F., Carlier A., Hebels D., Dede-Eren A., ten Dijcke P., Vasilevich A., de Boer J. Mechanotransduction is a context-dependent activator of TGF- $\beta$ signaling in mesenchymal stem cells. Submitted for publication

Vassey M., Figueredo G., Scurr D., Vasilevich A., Vermeulen S., Carlier A., Luckett J., Beijer N., Williams P., Winkler D., de Boer J., Ghaemmaghami A., Alexander M. Immune modulation by design: using topography to control human monocyte attachment and macrophage differentiation. Advanced Science 2020 (I.F.: 15.8) (PMID: 32537404) 
Vermeulen S., Vasilevich A., Tsiapalis D., Roumans N., Vroemen P., Beijer N., Dede-Eren A., Zeugolis D., de Boer J. Identification of Topographical Architectures Supporting the Phenotype of Rat Tenocytes. Acta Biomaterialia 2019 (I.F.: 7.24) (PMID: 30394345)

Leuning D., Beijer N., du Fosse N., Vermeulen S., Lievers E., van Kooten C., Rabelink T., de Boer J. The cytokine secretion profile of mesenchymal stromal cells is determined by the surface structure of the microenvironment. Scientific Reports 2018 (I.F.: 4.12) (PMID: 29769543)

Bach Q., Vasilevich A., Vermeulen S., Hulshof F., Stamatialis D., van Blitterswijk C., de Boer J. Micro-topographies promote late chondrogenic differentiation markers in the ATDC5 cell line. Tissue Engineering: Part A 2017 (I.F.: 3.68) (PMID: 28152670)

Van den Bossche T., Sleegers K., Cuyvers E., Engelborghs S., Sieben A., De Roeck A., Van Cauwenberghe C., Vermeulen S., Van den Broeck M., Laureys A., Peeters K., Mattheijssens M., Vandenbulcke M., Vandenberghe R., Martin JJ., De Deyn PP., Cras P., Van Broeckhoven C.; Belgian Neurology Consortium: Phenotypic characteristics of Alzheimer patients carrying an ABCA7 mutation. Neurology 2016 (I.F.: 8.69) (PMID: 27037232)

Bettens K., Vermeulen S., Van Cauwenberghe C., Heeman B., Asselbergh B., Robberecht C., Engelborghs S., Vandenbulcke M., Vandenberghe R., De Deyn P., Cruts M., Van Broeckhoven C. and Sleegers K.: Reduced secreted clusterin as a mechanism for Alzheimer-associated CLU mutations. Molecular Neurodegeneration 2015 (I.F.: 8.38) (PMID: 26179372)

Cuyvers E., De Roeck A., Van den Bossche T., Van Cauwenberghe C., Bettens K., Vermeulen S., Mattheijssens M., Peeters K., Engelborghs S., Vandenbulcke M., Vandenberghe R., De Deyn P., Van Broeckhoven C., Sleegers K.: Mutations in ABCA7 in a Belgian cohort of Alzheimer's disease patients: a targeted resequencing study. The Lancet Neurology 2015 (I.F.: 25.94) (PMID: 26141617)

Sleegers K., Bettens K., De Roeck A., Van Cauwenberghe C., Cuyvers E., Verheijen J., Struys H., Van Dongen J., Vermeulen S., Engelborghs S., Vandenbulcke M., Vandenberge R., De Deyn P, Van Broeckhoven C., on behalf of the BELNEU consortium: A 22-SNP Alzheimer risk score correlates with family history, onset age and CSF A 342 . Alzheimer's and Dementia 2015 (I.F.: 12.74) (PMID: 26086184)

Van Cauwenberghe C., Bettens K., Engelborghs S., Vandenbulcke M., Van Dongen J., Vermeulen S., Vandenberghe E., De Deyn P., Van Broeckhoven C., Sleegers K.: CR1 coding variant p.Ser160Thr in Alzheimer's disease and related endophenotypes. Neurobiology of Aging 2013 (I.F.: 4.39) (PMID: 23582656)

Bettens K., Brouwers N., Engelborghs S., Lambert JL., Rogaeva E., Vandenberghe R., Le Bastard N., Pasquier F., Vermeulen S., Van Dongen J., Mattheijssens M., Peeters K., Mayeux R., St George-Hyslop P., Amouyel P., De Deyn P., Sleegers K. and Van Broeckhoven C.: Both common variations and rare non-synonymous substitutions and small insertion/deletions in CLU are associated with increased Alzheimer risk. Molecular Neurodegeneration 2012 (I.F.: 8.38) (PMID: 22248099) 
Gijselinck I., Van Langenhove T., van der Zee J., Sleegers K., Philtjens S., Kleinberger G., Janssens J., Bettens K., van Cauwenberghe C., Pereson S., Engelborghs S., Sieben A., De Jonghe P., Vandenberghe R., Santens P., De Bleecker J., Maes G., Bäumer V., Dillen L., Joris G., Cuijt I., Corsmit E., Elinck E., Van Dongen J., Vermeulen S., Van den Broeck M., Vaerenberg C., Mattheijssens M., Peeters K., Robberecht W., Cras P., Martin J., De Deyn P., Cruts M., Van Broeckhoven C.: C9orf72 promotor repeat expansion in a Flanders - Belgian cohort with disorders of the frontotemporal lobar degeneration-amyotrophic lateral sclerosis spectrum: a gene identification study. The Lancet Neurology 2012 (I.F.: 25.94) (PMID: 22154785)

\section{Review Articles in International Journals}

Vermeulen S., de Boer J. Modulation of biochemical signaling through shaping the cells mechanical environment. Manuscript in preparation.

Honig F., Vermeulen S., Zadpoor A., de Boer J., Fratila-Apachitei L. Natural Architectures for Tissue Engineering and Regenerative Medicine. Journal of Functional Biomaterials (I.F.: 3.27) Accepted for publication

Vermeulen S., de Boer J. Screening as a strategy to drive regenerative medicine research. Methods 2020 (I.F.: 3.81) (PMID: 32278807)

Kumari S.* Vermeulen S.*, van der Veer B., Carlier A., de Boer J and Subramanyam D. Shaping cell fate: influence of substratum properties on embryonic stem cells. Tissue Engineering: Part B 2018 (I.F: 7.07) (PMID: 29455619)

* These authors contributed equally

\section{Scientific Communications}

\section{Poster Presentations}

Vermeulen S., Floris H., Roumans N., Dede A, de Boer J. Topography-Induced Mechanotransduction is a Context-Dependent Regulator of Stem Cell Differentiation. BME (2019)

Vermeulen S., Vasilevich A, Dimitrios T, Dede A, Zeugolis D, de Boer J. Engineering the tendon fibroblast micro-environment by surface topographies. ESB (2017)

Vasilevich A., Vermeulen S., Kamphuis M., Beijer N., Carlier A., Singh S., de Boer J. Does cell shape direct cell physiology? NBTE (2016)

Hebels D., Vermeulen S., de Boer J. Distinct transcriptional profiles controlled by chemistry and surface topography in calcium phosphate ceramics. ISSCR (2016) 


\section{Oral Presentations}

Vermeulen S., Roumans N., Honig F., Dede A, Carlier A., ten Dijke P., de Boer J. TopographyInduced Mechanotransduction is a Context-Dependent Regulator of Stem Cell Differentiation. MERLN PhD Symposium (2019)

Vermeulen S., Dede A., Honig F., Roumans N., de Boer J. Topography-Induced Mechanotransduction is a Context-Dependent Regulator of Stem Cell Differentiation. MERLN PhD Symposium (2018)

Vermeulen S., Dede A., Honig F., Roumans N., de Boer J. Topography-Induced Mechanotransduction is a Context-Dependent Regulator of Stem Cell Differentiation. CytoData Symposium (2018)

Vermeulen S., Dede A., Honig F., Roumans N., de Boer J. Topography-Induced Mechanotransduction is a Context-Dependent Regulator of Stem Cell Differentiation. Nikon High-Throughput Technologies Symposium (2018)

Vermeulen S., Dede A., Honig F., Roumans N., de Boer J. Topography-Induced Mechanotransduction is a Context-Dependent Regulator of Stem Cell Differentiation. ESB (2018)

Vermeulen S., Honig F., de Boer J. Natural and Artificial Surface Architectures to Control Stem Cell Fate. Quito - Axxes Hospital (2018)

Vermeulen S., Vasilevich A., Dimitrios T., Dede A., Zeugolis D., de Boer J. Identification of Micro-Topographies Supporting the Phenotypical Maintenance of Rat Tenocytes. MERLN PhD Symposium (2017)

Vermeulen S., Vasilevich A., Dimitrios T., Dede A., Zeugolis D., de Boer J. Engineering the tendon fibroblast micro-environment by surface topographies. ESB (2017) 


\section{Education Activities}

\section{Tutoring}

PRA3017 - Applied Cell Biology - Master Biomedical Sciences (2016-2019)

MBS 1304 - Master Biomedical Sciences (2017- 2018)

BIO1001 - Biology - Bachelor Biomedical Sciences (2017)

\section{Supervisor Master Internships}

Laura Bengtsson del Barrio (2019) Regulation of Mesenchymal Stem Cell Nuclear Architecture by Micro-Topographies

Floris Honig (2017-2018) Biomimicry: Natural topographies to control cell behavior

Ben van der Veer (2016-2017) The effect of micro-topography on global histone acetylation and methylation in human mesenchymal stem cells 


\section{Awards}

Award from the MERLN PhD Symposium for Best Oral Presentation 2018.

Award from the European Society for Biomaterials for Best Oral Presentation 2018.

Travel Grant CytoData Symposium Boston 2018. 


\section{Acknowledgments}

The past years have been quite the journey. A unique and incredible one that would not have been possible without the support of quite a few people, which I hope to thank here.

Foremost, I would like to thank you Jan for allowing me to start a $\mathrm{PhD}$ in your group. The experience allowed developing myself, both scientifically and personally. I am very happy with the opportunities that you gave me in the group, yet also abroad through collaborations with other research labs. I genuinely appreciated all the scientific and non-scientific discussions we had (supplemented with the occasional Dutch quote), and ensuring that the cBITE/BiS group was both a professional and pleasant environment to work in. I hope to continue seeing some form of collaboration (or sailing trip) in the future! Aurélie, similar to Jan, I enjoyed all the discussions we had and appreciated you as a member of the team. As a "pure" computational member, it was sometimes difficult to understand what the meaning was behind all those equations that passed by. Nevertheless, you showed everyone what a great teacher you are through the "bunny and foxes" presentation, allowing us to read differential formulas. Thank you for being my co-supervisor and all the valuable advice you gave during my $\mathrm{PhD}$ !

Alex, thank you for all the computation input that I received from you in the past years. Learning how to properly code in $\mathrm{R}$ to master high-throughput TopoChip analysis became less intimidating thanks to you. I enjoyed all the scientific and social discussions we had, and I wish you the best in your future endeavors! Aysegul, my tendon counterpart in the consortium project, thanks for being such a cheerful colleague in the lab, office, and beyond. The trips to Galway would not have been so much fun without you. And, after your leaving towards Eindhoven, I genuinely missed the coffee breaks and your ability to elevate conversations to a new level of "geekiness". I wish you and Deniz all the best in your future adventures! Pascal, the eye specialist of the group, you taught me how to properly isolate tendon tissue, of which the result can be seen in this thesis. Thanks for being an enjoyable colleague, and I hope for a smooth completion of your PhD. Nadia, also you contributed quite substantially to the work described in this thesis, and I can't thank you enough for it. MERLN should be very happy to have you around! Jasia, as no one else, you translate computational work into practice, and I am curious to see which great results will come from that. You taught me that Belgium is not the only country in the world that can process (great) chocolate, and it is good to know that if I ever venture into Saint Lucia, I will have a guide to show me around (or at least keep me safe). Dennie, in the past years you were the mastermind behind many events, both social and professional ones. Thank you for all that organization and for being a great member of the team! Saïd, you must be one of the most sportive people of the institute. Keep it up! Also thanks again for all the nice gifts from your home country and introducing me goat as a delicacy. Urnaa, I enjoyed the scientific and social discussions we had. I hope for a smooth completion of your $\mathrm{PhD}$ and the best with Suvd and your partner! Nick, it is already quite some time ago that you 
completed your PhD and ventured into a new job. I enjoyed all our trips to the clean room in Twente, which made us the essential individuals that allowed cBITE to perform research. I appreciated learning how to work there and all the social activities surrounding it! Christine, also for you it is already quite some time that you left the MERLN environment. Thank you for always lending a helping hand, which also turned out to have excellent squashing skills. Marloes, also you obliterated me during squashing events, so I appreciate you much more for doing an excellent job in keeping everything running smoothly in the labs. Indirectly, you contributed to this work by allowing us to work in a clean and organized environment making everyday life in the labs easier. Thank you for that! Jorge, thanks for all the hospitality and guidance in Ecuador! It was truly a nice experience, and it pleases me that the collaborations eventually led you to move to the Netherlands. I wish you the best in your future career in Jan's group! Phani, Sultan, Nusrat, Katinka, unfortunately, due to the movement of Jan to Eindhoven, I did not have a chance to get to know you better. Nevertheless, I enjoyed the discussions we had, and I wish you the best with your future careers!

In the past years, the MERLN institute has grown quite some numbers. This sometimes caused me to address some people with "hey" instead of with your name. My apologies for that. Nevertheless, I enjoyed the high degree of professionalism and collegiality in the institute, and thank you all for contributing to that. Especially for Paul and Timo for discussing microscopy issues, image analysis discussions, and organizing our Nikon trip to Prague. And Stefan, Roman for all manufacturing related topics.

A special thanks to my family. Voor mijn ouders, bedankt voor jullie steun tijdens en ook vóór mijn PhD periode. Zowel Annelynn en ik apprecieerden het dat we het ouderlijk huis af en toe als een kosteloze Airbnb konden gebruiken om van Antwerpen in de weekends te genieten. Sven en Co, ik hoop om jullie in post-coronatijden wat vaker te zien! Carine en Patrick, bedankt voor de gezelligheid elke keer opnieuw! Olaf, Dirk, Kevin en Steven, op nog veel Mtg/Scythe/GoT avonden! Annelynn, bedankt om samen dit avontuur met me aan te gaan. De voorbije jaren waren druk, maar hoop dat je toch ten volle hebt kunnen genieten van de verre reisjes die we deden. Bedankt om er telkens voor me te zijn, dit werk is door de vele steun ook jouw verdienste!

Lastly, I would like to thank the assessment commission, Prof. Dr. Pamela Habibović, Prof. Dr. Amir Ghaemmaghami, Prof. Dr. Carlijn Bouten, Prof. Dr. Clemens van Blitterswijk, and Dr. Pieter Emans for their time and critical assessment of this thesis. 


\section{Biography}

Steven Vermeulen was born on the 28th of August 1986 in Antwerpen, Belgium. Steven finished a master's degree in industrial engineering, specialization biochemistry in 2010. Afterward, Steven got an opportunity to work at the molecular genetics department of the VIB in Antwerp. In the research lab of Prof. Christine van Broeckhoven, Steven assisted in finding novel mutations associated with Alzheimer's disease and elucidating their phenotypic consequences. In 2016, Steven embarked on a new adventure in the form of a $\mathrm{PhD}$ project at the MERLN institute. Under the supervision of Prof. Jan de Boer, Steven researched how physical cues in the form of micro-topographies and natural surface structures alters the phenotype of cells, of which the results are described in this thesis.

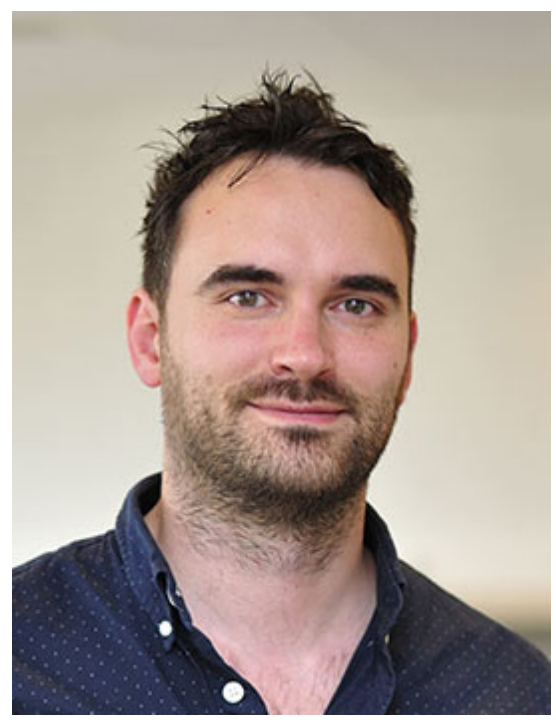

